\title{
Estruturas de Vertex em teoria de representações de álgebras de Lie
}

\author{
Renato Alessandro Martins
}

TESE APRESENTADA

$\mathrm{AO}$

Instituto DE MATEMÁticA E EstatísticA

DA

UniversidAde DE SÃO PAUlO

PARA

OBTENÇÃO DO TÍTULO

DE

Doutor EM CIÊNCIAS

\author{
Programa: Matemática \\ Orientador: Prof. Dr. Vyacheslav Futorny
}

Durante o desenvolvimento deste trabalho o autor recebeu auxílio financeiro da FAPESP (Processo: 2008/06860-3)

São Paulo, maio de 2012 



\section{Estruturas de Vertex em teoria de representações de álgebras de Lie}

Esta versão da tese contém as correções e alterações sugeridas pela Comissão Julgadora durante a defesa da versão original do trabalho, realizada em 04/05/2012. Uma cópia da versão original está disponível no Instituto de Matemática e Estatística da Universidade de São Paulo.

Comissão Julgadora:

- Prof. Dr. Vyacheslav Futorny - IME-USP

- Prof. Dr. Henrique Guzzo Júnior - IME-USP

- Prof. Dr. Reimundo Heluani - IMPA

- Prof. Dr. Plamen Emilov Kochloukov - UNICAMP

- Prof. Dr. Dimitar Grantcharov - Univ. of Texas 



\section{Agradecimentos}

Gostaria de agradecer primeiramente os meus pais por todo o apoio que recebi durante minha longa jornada até a conclusão desta tese de doutorado.

Também gostaria de agradecer meu orientador, Prof. Dr. Vyacheslav Futorny, por ter acreditado no meu potencial desde o início, me dando a oportunidade de desenvolver este projeto. Saiba que trabalhar com um pesquisador de altíssimo nível como você foi fundamental para meu crescimento e amadurecimento como matemático.

Agradeço também aos meus amigos que sempre me apoiaram mesmo naqueles momentos mais difíceis deste doutorado. Aos meus colegas que estavam torcendo para que tudo terminasse bem, meus sinceros agradecimentos.

Agradeço a todos os bons professores que se esforçaram para contribuir positivamente em minha formação, sendo exigentes na hora que tinham que ser exigentes, e motivadores nos momentos de maior dificuldade.

Agradeço também a FAPESP por ter financiado este projeto de pesquisa. 


\section{Resumo}

Motivados pelos resultados do artigo [BBFK11], nosso trabalho começa analisando, no caso da álgebra de Lie afim $\widehat{\mathfrak{s l}}(n, \mathbb{C})$, a possibilidade de se obter módulos de Verma J-imaginários, via representações análogas às feitas por Cox em [Cox05]. Inicialmente consideramos, por simplicidade, $n=2$ e, só então, analisamos o caso geral. Depois, de modo análogo, estudamos os artigos [CF04] e [CF05] com o intuito de obter módulos J-intermediários de Wakimoto.

Finalmente imbutimos, no caso $n=2$, uma ação de álgebra de Virasoro nos módulos imaginários de Wakimoto, utilizando-nos do resultado exposto em [EFK98], em que tal problema é abordado para o caso dos módulos de Verma. Desta forma, obtemos equações análogas às de Knizhnik-Zamolodchikov (equações KZ) para os módulos imaginários de Wakimoto.

Palavras-chave: módulos de Verma clássicos, módulos de Verma imaginários, módulos de Verma J-imaginários, módulos intermediários de Wakimoto, módulos J-intermediários de Wakimoto, álgebras de Vertex. 


\section{Abstract}

Following the results of [BBFK11], our work starts analyzing (for $\widehat{\mathfrak{s l}}(n, \mathbb{C})$ ) if we can obtain J-imaginary Verma modules using similar representations used by Cox in [Cox05]. We did it for $n=2$ and after, for the general case. The next step was the study of $\mathbb{J}$-intermediate Wakimoto modules, following the ideas of [CF04] and [CF05].

To finish, for $\widehat{\mathfrak{s l}}(2, \mathbb{C})$, we defined an action of Virasoro algebra on the imaginary Wakimoto modules following [EFK98] and we obtained an analogue of the KZ-equations for imaginary Wakimoto modules.

Keywords: classical Verma modules, imaginary Verma modules, $\mathbb{J}$-imaginary Verma modules, intermediate Wakimoto modules, J-intermediate Wakimoto modules, Vertex algebras. 


\section{Sumário}

1 Definições básicas 1

1.1 Distribuições Formais . . . . . . . . . . . . . . . . . . . . . 1

1.2 Álgebras de Lie . . . . . . . . . . . . . . . . . . . . . . . 4

1.3 Os espaços de Fock . . . . . . . . . . . . . . . . . . . 5

1.4 Os módulos do tipo Verma e os $\mathbb{J}$-módulos do tipo Verma $\ldots \ldots . \ldots$

1.4.1 Primeira realização de campo livre . . . . . . . . . . . . . . 7

1.4.2 Módulos de Wakimoto . . . . . . . . . . . . . . . . . . . . 8

1.4.3 Módulos intermediários de Wakimoto . . . . . . . . . . . . . . . . 9

2 Os módulos de Verma J-imaginários $\quad 11$

2.1 Introdução . . . . . . . . . . . . . . . . . . . . . . . . . . . 11

2.2 Realização de campos livres de $\widehat{\mathfrak{s l}}(n, \mathbb{C}) \ldots \ldots \ldots \ldots . \ldots \ldots$

2.2 .1 O caso $n=2 \ldots \ldots \ldots \ldots \ldots \ldots$

$2.2 .2 \quad \mathrm{O}$ caso $n>2 \ldots \ldots \ldots \ldots \ldots$

3 Os módulos 『-intermediários de Wakimoto 33

3.1 Os módulos intermediários de Wakimoto . . . . . . . . . . . . . . 33

3.1 .1 Álgebra de oscilação . . . . . . . . . . . . . . . . . . . 34

3.1 .2 Cálculos de campos . . . . . . . . . . . . . . . . . . 38

3.1 .3 A representação . . . . . . . . . . . . . . . . . . . . . 48

3.2 Construção dos módulos J-intermediários de Wakimoto . . . . . . . . . . . . 59

3.2 .1 As J-realizações boson . . . . . . . . . . . . . . . . . . . . . . . . . 59

3.3 Gerando módulos đJ-intermediários de Wakimoto . . . . . . . . . . . . . 64

3.4 Módulos $\left(\mathbb{J}_{1}, \mathbb{J}_{2}\right)$-intermediários de Wakimoto . . . . . . . . . . . . . 67

4 A ação de Virasoro nos módulos imaginários de Wakimoto para $\widehat{\mathfrak{s l}}(2, \mathbb{C}) \quad 71$

4.1 Introdução . . . . . . . . . . . . . . . . . . . . . . . 71

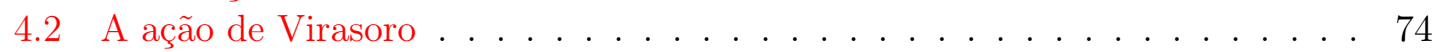

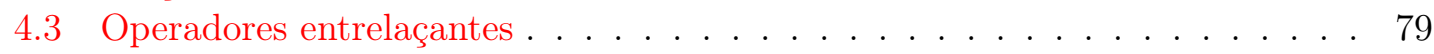

4.3.1 Módulos Topológicos . . . . . . . . . . . . . . . . . . . 79

4.3.2 Forma operadora das equações de Knizhnik-Zamolodchikov . . . . . 84

5 Considerações finais $\quad 91$ 


\section{Introdução}

As álgebras de Kac-Moody desempenham um importante papel para diversos segmentos da Matemática e da Física. Tais álgebras foram introduzidas em 1968 por V. Kac e R. Moody como uma generalização das álgebras de Lie simples de dimensão finita com matriz de Cartan positiva e definida. As álgebras de Lie afim são as mais estudadas das álgebras de Kac-Moody de dimensão infinita e possuem importantes aplicações. Estas correspondem ao caso das matrizes positivas semidefinidas $\left(\operatorname{det}\left(a_{i j}\right)=0\right.$ com menores principais positivos). Uma maneira de se construir representações de álgebras de Lie afim é via indução das subálgebras parabólicas. Temos que os módulos de indução possuem um papel importante no problema da classificação de módulos irredutíveis.

A teoria dos módulos de Verma para álgebras de Lie afim foram desenvolvidas em diferentes trabalhos, como por exemplo, em [Kac90] e em [DGK82]. No caso de dimensão infinita, sabe-se que nem todas as subálgebras de Borel são conjugadas com respeito ao grupo de Weyl para álgebras de Lie afim. Neste caso, existe mais de uma (mas ainda em quantidade finita) classe de conjugação de subálgebras de Borel que nos leva a construção de módulos tipo Verma. Temos que todas as subálgebras de Borel foram classificadas em [JK85] e [JK89] e, independentemente, em [Fut90] e [Fut91]. Sabemos que os módulos de Verma que correspondem a uma subálgebra de Borel não-standard são bem diferentes dos módulos de Verma clássicos. À saber, eles possuem espaços de peso tanto de dimensão finita quanto infinita.

Em 1985, H. Jacobsen e V. Kac [JK85] apresentaram novas classes de representações de peso máximo unitarizável para álgebras de Lie de dimensão infinita, a qual denominamos módulos de Verma imaginários e, em 1986, M. Wakimoto [Wak86] nos deu uma

representação de Fock para a álgebra de Lie afim $A_{1}^{(1)}$. Nestes trabalhos foram feitas construções geométricas de certas representações de álgebras de Kac-Moody afim em termos de somas infinitas de operadores diferenciais parciais agindo num espaço de Fock. Em 1990, D. Bernard e G. Felder nos deram uma representação no espaço de Fock de módulos de Verma imaginários para $\widehat{\mathfrak{s l}}(2, \mathbb{C})$ o qual foi generalizado em 2005 por B. Cox [Cox05] para $\widehat{\mathfrak{s l}}(n, \mathbb{C})$. Em 2010, generalizamos os resultados obtidos por B. Cox em [Cox05] para os módulos $\mathbb{J}$-imaginários (ver [Mar11]). Em 1994, V. Futorny estudou os módulos de Verma imaginários para álgebras de Lie afim (ver [Fut94]) enquanto que B. Cox trabalhou com módulos de Verma induzidos de subálgebras de Borel não-standard (ver [Cox94]). Em 2004 e 2005, B. Cox e V. Futorny nos deram realizações para os módulos intermediários de Wakimoto (ver [CF04] e [CF05]), cuja generalização para módulos J-intermediários de Wakimoto foi feita em 2011 (ver [Mar12]).

A teoria de módulos de Verma generalizados com peso máximo foi estudada inicialmente por A. Rocha e N. Wallach em [RW82]. Estes módulos podem ser vistos como induzidos de uma subálgebra parabólica com um fator de Levi redutível de dimensão finita. Todas 
as subálgebras parabólicas no caso afim foram descritas em [Fut90] e [Fut91]. Agora existe um número finito de classes de conjugação, com respeito ao grupo de Weyl, de subálgebras parabólicas com fator de Levy redutível fixado. Todas as subálgebras parabólicas definem um módulo generalizado do tipo Verma. Um fato relacionado às álgebras de Lie afim é a existência de subálgebras parabólicas com um fator de Levi contendo uma subálgebra de Heisenberg de dimensão infinita ou uma álgebra de Lie afim com posto menor. Módulos induzidos no primeiro caso são chamados de módulos de Loop. Em particular, representações adjuntas podem ser obtidas como quociente de um módulo de Loop. Módulos de Loop de nível zero foram estudados em [C86], [R89], [R93], [CP88] e [CP088]. Módulos induzidos de uma subálgebra parabólica contendo uma subálgebra de Lie afim são chamados módulos de Loop generalizados. Os módulos tipo Verma são deste tipo.

Quanto à organização desta tese, o capítulo 1 tem como objetivos introduzir os conceitos básicos abordados neste texto e familiarizar o leitor com as notações adotadas. Algumas definições como a de distribuições formais e de módulos de Verma clássicos e imaginários, além dos módulos intermediários de Wakimoto, são expostos neste capítulo devido à sua importância e utilidade ao longo do trabalho.

Já o capítulo 2 aborda os resultados obtidos pelo autor em [Mar11], onde estuda-se, para as álgebras de Lie afim $\widehat{\mathfrak{s l}}(n, \mathbb{C})$, uma realização em termos de somas infinitas de operadores diferenciais parciais de uma família de representações introduzidas no artigo [BBFK11], representações estas que generalizam as construções de módulos de Verma imaginários. Desta forma, generalizamos as realizações de campos livres de módulos de Verma imaginários construídos por B. Cox em [Cox05] para todos os módulos de Verma $\mathbb{J}$-imaginários sobre a álgebra de Lie afim $\widehat{\mathfrak{s l}}(n, \mathbb{C})$.

No capítulo 3, apresentamos, na primeira seção, os resultados obtidos por B. Cox e V. Futorny em [CF04] e [CF05]. Em seguida, na seção 3.2, aborda-se o conteúdo de [Mar12], em que se generaliza as realizações dos módulos intermediários de Wakimoto apresentadas nestes dois trabalhos para todos os módulos J-intermediários de Wakimoto. Vale ressaltar que nestes trabalhos de Cox e Futorny os módulos dependem de um parâmetro $r, 0 \leqslant r \leqslant n$, onde, para $r=0$, temos [Cox05] e, para $r=n$, o resultado coincide com a das realizações bóson dos módulos de Wakimoto vistas em [FF88]. Desta forma, nosso trabalho generaliza conseqüentemente tanto [Mar11] quanto [FF88].

No capítulo 4, apresentamos os resultados obtidos em [CFMar12], o qual aborda a ação de álgebra de Virasoro sobre os módulos intermediários de Wakimoto estudados em [CF04] e [CF05] para o caso de $\widehat{\mathfrak{s l}}(2, \mathbb{C})$. Neste capítulo, apresentamos uma equação análoga à de Knizhnik-Zamolodchikov (ver [EFK98]).

Finalmente, no capítulo 5, damos uma breve introdução ao que pretendemos estudar daqui em diante. 


\section{Capítulo 1}

\section{Definições básicas}

Este primeiro capítulo tem os objetivos de familiarizar o leitor com os conceitos básicos relativos à teoria e estabelecer as notações que serão utilizadas no decorrer desta tese. Assumimos que o leitor possui uma noção básica sobre a teoria de álgebras de Lie, noções estas contidas em [SM99]. Outros conceitos não tão básicos, como o de distribuições formais, produto ordenado normal, módulos de Verma clássicos e imaginários, além dos módulos de Wakimoto e intermediário de Wakimoto, são abordados neste primeiro capítulo devido a sua importância no desenvolvimento da teoria. Os conceitos mais específicos são deixados para os capítulos subseqüêntes, aparecendo somente quando realmente necessários. Uma exceção a esta "regra" é o conceito de J-módulos tipo Verma, já que sua compreensão é indispensável no estudo dos resultados expostos em toda a tese. Devido à importância dada pelos físicos, também discutimos brevemente o conceito de espaço de Fock, uma vez que fazemos referência a tal assunto nos capítulos 2 e 3, deixando a cargo do leitor uma leitura mais profunda do assunto caso seja de seu interesse.

Desta forma, iniciamos nossos estudos abordando os conceitos e os resultados básicos relativos à teoria de distribuições formais.

\subsection{Distribuições Formais}

Definição 1.1.1. Seja $U$ um espaço vetorial sobre $\mathbb{C}$. Chamemos de distribuição formal nas indeterminadas $z_{1}, z_{2}, \ldots, z_{n}$ com valores em $U$ às séries da forma

$$
a\left(z_{1}, z_{2}, \ldots, z_{n}\right)=\sum_{i_{1}, \ldots, i_{n}=-\infty}^{\infty} a_{i_{1}, \ldots, i_{n}} \cdot z_{1}^{i_{1}} \cdot \ldots \cdot z_{n}^{i_{n}}
$$

onde $a_{i_{1}, \ldots, i_{n}} \in U$ para todo $i_{1}, \ldots, i_{n} \in \mathbb{Z}$.

Estas distribuições formais formam um espaço vetorial sobre $\mathbb{C}$ denotado por $U\left[\left[z_{1}, z_{1}^{-1}, \ldots, z_{n}, z_{n}^{-1}\right]\right]$. Aqui a utilização dos dois colchetes é necessária, já que utiliza-se um colchete apenas quando o número de termos for finito. Por exemplo, $\left.a(z) \in \mathbb{C}\left[z^{-1}, z\right]\right]$ significa que $a(z)$ é uma distribuição formal com quantidade finita de potências negativas.

Observação 1.1.3. Nem sempre duas distribuições formais podem ser multiplicadas. Para tal propósito, é necessário verificar sua convergência no sentido algébrico, isto é, se os 
coeficientes de cada monômio $z_{1}^{i_{1}} \ldots z_{n}^{i_{n}}$ são somas convergentes. Por exemplo, quando uma das distribuições formais é, na verdade, um polinômio de Laurent, então o produto está bem definido, já que cada coeficiente $a_{i_{1}, \ldots, i_{n}}$ é obtido por uma soma finita, logo convergente.

Definição 1.1.4. Seja $a(z)=\sum_{n \in \mathbb{Z}} a_{n} z^{n} \in U\left[\left[z, z^{-1}\right]\right]$. Define-se o resíduo de $a(z)$, e o denota-se por $\operatorname{Res}_{z} a(z)$, como o coeficiente $a_{-1}$ de $a(z)$, isto é,

$$
\operatorname{Res}_{z} a(z)=a_{-1}
$$

e também seja $\partial a(z)$ a derivada de a(z) na variável $z$, isto é,

$$
\partial a(z)=\sum_{n \in \mathbb{Z}} n \cdot a_{n} z^{n-1} .
$$

Assim, quando o produto $a(z) b(z)$ está bem definido, tem-se a igualdade

$$
\operatorname{Res}_{z} \partial a(z) b(z)=-\operatorname{Res}_{z} a(z) \partial b(z),
$$

onde $\partial a(z) b(z)$ significa $(\partial a(z)) \cdot b(z)$ e $\operatorname{Res}_{z} a(z) b(z)$ significa $\operatorname{Res}_{z}(a(z) \cdot b(z))$. De fato, como

$$
\partial(a(z) b(z))=\partial a(z) b(z)+a(z) \partial b(z),
$$

e $\operatorname{Res}_{z} \partial c(z)=0$, tem-se

$$
\begin{aligned}
0 & =\operatorname{Res}_{z} \partial(a(z) b(z)) \\
& =\operatorname{Res}_{z}(\partial a(z) b(z)+a(z) \partial b(z)) \\
& =\operatorname{Res}_{z} \partial a(z) b(z)+\operatorname{Res}_{z} a(z) \partial b(z) .
\end{aligned}
$$

Denote a álgebra dos polinômios de Laurent em $z$ por $\mathbb{C}\left[z, z^{-1}\right]$. Temos a seguinte definição.

Definição 1.1.6. Sejam $f(z) \in U\left[\left[z, z^{-1}\right]\right]$ e $\varphi(z) \in \mathbb{C}\left[z, z^{-1}\right]$. Temos a função

$$
\begin{aligned}
& \langle\cdot, \cdot\rangle: \quad U\left[\left[z, z^{-1}\right]\right] \times \mathbb{C}\left[z, z^{-1}\right] \quad \longrightarrow \quad U \\
& (f, \varphi) \quad \longmapsto \operatorname{Res}_{z} f(z) \varphi(z) .
\end{aligned}
$$

O polinômio $\varphi(z) \in \mathbb{C}\left[z, z^{-1}\right]$ é chamado, neste caso, de função de teste. Vale o seguinte teorema.

Teorema 1.1.7. Sejam $a(z), b(z) \in U\left[\left[z, z^{-1}\right]\right]$. Então as distribuições formais $a(z) e$ $b(z)$ são iguais se, e somente se, para toda função de teste $\varphi(z) \in \mathbb{C}\left[z, z^{-1}\right]$, valer que $\langle a, \varphi\rangle=\langle b, \varphi\rangle$ (onde $a=a(z)$ e $b=b(z))$.

Demonstração. De fato, $a(z)=b(z)$ implica que $\langle a, \varphi\rangle=\langle b, \varphi\rangle$. Assim, basta provar que

$$
\langle a, \varphi\rangle=\langle b, \varphi\rangle \text {, para todo } \varphi \text {, implica } a(z)=b(z) .
$$

Fixado $m$, defina $\varphi_{m}=z^{-m-1}$. Então 


$$
\begin{aligned}
\left\langle a, \varphi_{m}\right\rangle & =\operatorname{Res}_{z} a(z) \varphi_{m}(z) \\
& =\operatorname{Res}_{z} a(z) \\
& =\operatorname{Res}_{z}\left(\sum_{n \in \mathbb{Z}} a_{n} \cdot z^{n} \cdot z^{-m-1}\right) \\
& =a_{m} .
\end{aligned}
$$

Analogamente, temos que $\left\langle b, \varphi_{m}\right\rangle=b_{m}$. Logo $a_{m}=b_{m}$, para todo $m \in \mathbb{N}$, e assim $a(z)=b(z)$.

Definição 1.1.8. Seja $\delta(z / w) \in U\left[\left[z, z^{-1}, w, w^{-1}\right]\right]$ a distribuição formal (denominada função formal delta) definida por

$$
\delta(z / w)=w^{-1} \sum_{n \in \mathbb{Z}}\left(\frac{z}{w}\right)^{n}=\sum_{n \in \mathbb{Z}} z^{n} w^{-n-1} .
$$

A função formal delta possui algumas propriedades importantes que serão demonstradas através da seguinte construção.

Sejam $C=\mathbb{C}\left[\left[z, z^{-1}, w, w^{-1}\right]\right]$ e $S=(z-w)^{n}$ tal que $n \geq 0$. O anel de frações de $C$ com respeito à $S$ (denotado por $S^{-1} C$ ) consiste das funções racionais da forma

$$
\frac{f(z, w)}{(z-w)^{n}}
$$

onde $f=f(z, w) \in C$ e $n \geq 0$. As derivações $\partial_{z}$ e $\partial_{w}$ agem naturalmente neste anel de acordo com a regra do quociente para diferenciações. Por exemplo,

$$
\partial_{z} \frac{f(z, w)}{(z-w)^{n}}=\frac{\partial_{z} f(z, w) \cdot(z-w)-n \cdot f(z, w)}{(z-w)^{n+1}} .
$$

Denote por $i_{z, w}$ e $i_{w, z}$ a inclusão em $C$ das séries de potências dos anéis $\mathbb{C}\left[\left[\frac{w}{z}\right]\right] \mathrm{e}$ $\mathbb{C}\left[\left[\frac{z}{w}\right]\right]$, respectivamente. Observe que

$$
(z-w) \cdot z^{-1} \cdot\left(1+\frac{w}{z}+\ldots+\left(\frac{w}{z}\right)^{k}+\ldots\right)=1 \text { em } \mathbb{C}\left[\left[\frac{w}{z}\right]\right]
$$

e que

$$
(z-w) \cdot w^{-1} \cdot\left(1+\frac{z}{w}+\ldots+\left(\frac{z}{w}\right)^{k}+\ldots\right)=1 \text { em } \mathbb{C}\left[\left[\frac{z}{w}\right]\right]
$$

Temos

$$
i_{z, w} \frac{1}{z-w}=z^{-1} \cdot \sum_{m=0}^{\infty}\left(\frac{w}{z}\right)^{m}=\sum_{m=0}^{\infty} z^{-m-1} w^{m}
$$

e também

$$
i_{w, z} \frac{1}{z-w}=-w^{-1} \cdot \sum_{m=0}^{\infty}\left(\frac{z}{w}\right)^{m}=-\sum_{m=0}^{\infty} z^{m} w^{-m-1}=-\sum_{m=-1}^{-\infty} z^{-m-1} w^{m}
$$


Usando indução, prova-se que

$$
i_{z, w} \frac{1}{(z-w)^{j+1}}=\sum_{m=0}^{\infty}\left(\begin{array}{c}
m \\
j
\end{array}\right) z^{-m-1} w^{m-j}
$$

e também que

$$
i_{w, z} \frac{1}{(z-w)^{j+1}}=-\sum_{m=-1}^{-\infty}\left(\begin{array}{c}
m \\
j
\end{array}\right) z^{-m-1} w^{m-j} .
$$

O que fizemos em (1.1.10) é o seguinte:

$$
i_{z, w}: \mathbb{C}\left[\left[\frac{w}{z}\right]\right] \longrightarrow C \text { é tal que } \frac{f(z, w)}{(z-w)^{n}} \longmapsto f(z, w) \cdot(z-w)^{-n}
$$

Assim, tem-se uma série de potências $f(z, w)$ em $\mathbb{C}\left[\left[\frac{w}{z}\right]\right]$ e como $i_{z, w}$ é apenas a inclusão em $C$, segue que

$$
i_{z, w}\left(\frac{1}{z-w}\right)=i_{z, w}\left(z^{-1} \cdot \sum_{m=0}^{\infty}\left(\frac{w}{z}\right)^{m}\right)=i_{z, w} \sum_{m=0}^{\infty} w^{m} z^{-m-1}=\sum_{m=0}^{\infty} z^{-m-1} w^{m}
$$

Para facilitar os cálculos, pode-se utilizar o seguinte argumento (menos formal, porém mais utilizado)

$$
i_{z, w} \frac{1}{z-w}=\frac{1}{z} \cdot \frac{1}{1-\frac{w}{z}}=z^{-1} \cdot \sum_{m=0}^{\infty}\left(\frac{w}{z}\right)^{m}=\sum_{m=0}^{\infty} z^{-m-1} w^{m} .
$$

Para finalizar, tem-se que tanto $i_{z, w}$ quanto $i_{w, z}$ comutam com $\partial_{z}$ e com $\partial_{w}$. Por exemplo,

$$
\begin{aligned}
i_{z, w} \partial_{z} \frac{f(z, w)}{(z-w)^{n}} & =i_{z, w} \frac{\left(\partial_{z} f(z, w)\right) \cdot(z-w)-n \cdot f(z, w)}{(z-w)^{n+1}} \\
& =\left(\partial_{z} f\right) \cdot(z-w)^{-n}-n \cdot(z-w)^{-(n+1)} f \\
& =\partial_{z}\left(f \cdot(z-w)^{-n}\right) \\
& =\partial_{z} i_{z, w} \frac{f}{(z-w)^{n}} .
\end{aligned}
$$

\section{$1.2 \quad$ Álgebras de Lie}

Apenas para relembrar, daremos as seguintes definições (ver [SM99] para maiores detalhes).

Definição 1.2.1. Uma álgebra de Lie $\mathfrak{g}$ sobre $\mathbb{C}$ é um espaço vetorial que possui uma aplicação bilinear $[]:, \mathfrak{g} \times \mathfrak{g} \longrightarrow \mathfrak{g}$ chamada colchete de Lie, satisfazendo as seguintes relações:

(1) $[x, x]=0, \forall x \in \mathfrak{g}$

(2) $[x,[y, z]]+[y,[z, x]]+[z,[x, y]]=0, \forall x, y, z \in \mathfrak{g}$. 
Definição 1.2.2. Uma representação $\rho$ de uma álgebra de Lie $\mathfrak{g}$ é um homomorfismo de $\mathfrak{g}$ na álgebra linear geral de um espaço vetorial $V$, isto é,

$$
\rho: \mathfrak{g} \longrightarrow \mathfrak{g l}(V)
$$

tal que

$$
\rho([x, y])=\rho(x) \circ \rho(y)-\rho(y) \circ \rho(x)
$$

para todo $x, y \in \mathfrak{g}$.

Definição 1.2.3. Seja $\mathfrak{g}$ uma álgebra de Lie. Um espaço vetorial $V$, munido de uma operação $\mathfrak{g} \times V \longrightarrow V$ dada por $(x, v) \longrightarrow x \cdot v$ é chamado $\mathfrak{g}$-módulo se satisfaz as seguintes condições:

(1) $(a x+b y) \cdot v=a(x \cdot v)+b(y \cdot v)$,

(2) $x \cdot(a v+b w)=a(x \cdot v)+b(x \cdot w)$,

(3) $[x, y] \cdot v=x y \cdot v-y x \cdot v$,

$\forall x, y \in \mathfrak{g}, u, v \in V, a, b \in \mathbb{C}$.

Observação 1.2.4. A representação $\rho: \mathfrak{g} \longrightarrow \mathfrak{g l}(V)$ pode ser vista como um $\mathfrak{g}$-módulo via a ação $x \cdot v=\rho(x)(v)$ e, reciprocamente, dado um $\mathfrak{g}$-módulo $V$ e definindo $\rho(x)(v)=x \cdot v$, as propriedades $(1)-(3)$ definem uma representação $\rho: \mathfrak{g} \longrightarrow \mathfrak{g l}(V)$. Assim temos uma equivalência entre a linguagem de módulos e a de representações.

\subsection{Os espaços de Fock}

Nos textos de físico-matemática, o espaço de Fock $\mathfrak{F}$ é o espaço das representações naturais irredutíveis de uma álgebra (de Lie) de Heisenberg $\mathfrak{H}$ de dimensão infinita. À saber, $\mathfrak{F}$ é o anel de polinômios $\mathbb{C}\left[\mathbf{x}_{\mathbf{i}} \mid i \in \mathbb{N}^{*}\right]$ e $\mathfrak{H}$ é a álgebra de Lie gerada pelas derivações $\partial / \partial x_{i}$ e pelo operador multiplicação por $x_{i}$.

A cerca de 40 anos, percebeu-se que o espaço de Fock pode nos dar interessantes realizações de peso máximo de representações de uma álgebra de Lie de Kac-Moody afim $\widehat{\mathfrak{g}}$. Assim, $\widehat{\mathfrak{g}}$ possue uma subálgebra natural de Heisenberg $\mathfrak{p}$ chamada subálgebra principal e um $\widehat{\mathfrak{g}}$-módulo de peso máximo simples chamado representação básica de $\widehat{\mathfrak{g}}$, que mantêm-se irredutível quando restrito a p. Além disso, temos uma extensão principal $\mathfrak{p}$ da representação de espaço de Fock $\mathfrak{F}$ de $\widehat{\mathfrak{g}}$. Isto foi feito inicialmente por Lepowski e Wilson (ver [LW78]) para $\widehat{\mathfrak{g}}=\widehat{\mathfrak{s l}}(2, \mathbb{C})$. Os geradores de Chevalley de $\widehat{\mathfrak{s l}}(2, \mathbb{C})$ agem em $\mathfrak{F}$ via operadores diferenciais de grau infinito fortemente ligados aos operadores de Vertex inventados pelos físicos na teoria dos modelos ressonantes duais. Mais tarde, tais construções foram generalizadas para todas as álgebras de Lie $\widehat{\mathfrak{g}}$ de tipo $A, D$ e $E$. (ver [KKLW81]).

De forma indepente e com objetivos diferentes, resultados similares (na teoria de equações sóliton) foram obtidos por Date, Jimbo, Kashiwara e Miwa em [DJKM82] para álgebras de Lie afins clássicas. Neste trabalho, eles muniram $\mathfrak{F}$ com uma ação de álgebra de Lie afim de posto infinito e então a restringiram a várias subálgebras $\widehat{\mathfrak{g}}$ para obter suas representações básicas.

Neste trabalho não abordaremos de forma mais profunda a teoria relativa aos espaços de Fock devido a sua utilização restrita nesta tese. 


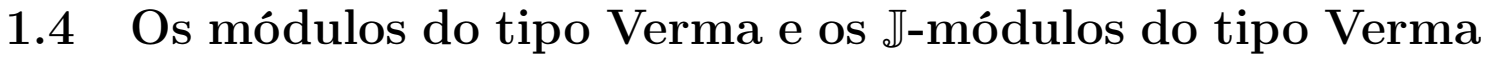

Seguindo as notações de [CF04] e [CF05], um subconjunto fechado $P \subset \Delta$ é chamado de partição se $P \cap(-P)=\varnothing$ e $P \cup(-P)=\Delta$. Para toda partição $P$ de $\Delta$, definimos a subalgebra de Borel $\mathfrak{b}_{P} \subset \mathfrak{s l}(n+1, \mathbb{C})$ gerada por $H$ e pelos espaços de raízes $a_{\alpha}$, com $\alpha \in P$.

Uma subálgebra parabólica é uma subálgebra que contém uma subálgebra de Borel. Se $\mathcal{P}$ é uma subálgebra parabólica de $\mathfrak{s l}(n+1, \mathbb{C})$ então $\mathcal{P}=\mathcal{P}_{0} \oplus \mathcal{P}_{+}$, onde $\mathcal{P}_{0}$ é um fator de Levi redutível e $\mathcal{P}_{+}$é uma subálgebra nilpotente. Subálgebras parabólicas correspondem a uma escolha de base $\Pi$ de um sistema de raízes $\Delta$ e um subconjunto $S \subset \Pi$ (ver [Fut97] e [DFG09] para maiores detalhes).

Seja $\mathcal{P}=\mathcal{P}_{0} \oplus \mathcal{P}_{+}$uma subálgebra parabólica de $\mathfrak{g}$ contendo uma fixada subálgebra de Borel $\mathfrak{b}$ de $\mathfrak{g}$. Seja

$$
B_{\mathcal{P}}=\left(\mathcal{P}_{+} \otimes \mathbb{C}\left[t, t^{-1}\right]\right) \oplus\left(\mathcal{P}_{0} \otimes t \mathbb{C}[t]\right) \oplus \mathfrak{b} \oplus \mathbb{C} c
$$

Então $\left\{B_{\mathcal{P}}\right\}$ parametriza classes de conjugação de subálgebras de Borel de $\widehat{\mathfrak{s l}}(n+1, \mathbb{C})$.

Definição 1.4.2. Seja $\lambda: B_{\mathcal{P}} \rightarrow \mathbb{C}$ uma representação unidimensional de $B_{\mathcal{P}}$. Então

$$
M_{\mathcal{P}}(\lambda)=U(\widehat{\mathfrak{g}}) \otimes_{U\left(B_{\mathcal{P}}\right)} \mathbb{C} v_{\lambda}
$$

é um $\widehat{\mathfrak{g}}$-módulo induzido do tipo Verma definido por $\lambda$.

Observação 1.4.4. Se $\mathcal{P}$ coincide com $\mathfrak{g}$ (isto é, $\mathcal{P}_{+}=0$ ) a correspondente subálgebra de Borel $B_{\mathfrak{g}}$ é a subálgebra de Borel standard definida pela escolha de raízes positivas em $\widehat{\mathfrak{g}}$. Neste caso, o correspondente $\widehat{\mathfrak{g}}$-módulo do tipo Verma

$$
M_{\mathfrak{g}}(\lambda)=U(\widehat{\mathfrak{g}}) \otimes_{U\left(B_{\mathfrak{g}}\right)} \mathbb{C} v_{\lambda}
$$

é o módulo de Verma clássico de peso máximo $\lambda$ (ver [Kac90]).

Observação 1.4.6. Se $\mathcal{P}_{0}=\mathfrak{H}$, temos a subálgebra de Borel natural $B_{\text {nat }}$ de $\widehat{\mathfrak{g}}$ estudada em [JK89] e

$$
M_{n a t}(\lambda)=U(\widehat{\mathfrak{g}}) \otimes_{U\left(B_{\text {nat }}\right)} \mathbb{C} v_{\lambda}
$$

é o módulo de Verma imaginário estudado em [Fut94].

Para $\Delta^{+}(r)=\Delta^{+} \cap \Delta\left(\mathfrak{g}_{r}\right)$, sejam

$$
\eta^{ \pm}=\oplus_{\alpha \in \Delta^{+}} \mathfrak{g}_{ \pm \alpha}, \eta_{r}^{ \pm}=\eta^{ \pm} \cap \mathfrak{g}_{r} \text { e } \eta^{ \pm}(r)=\oplus_{\alpha \in \Delta^{+} \backslash \Delta^{+}(r)} \mathfrak{g}_{\alpha} .
$$

Defina também

$$
\bar{B}_{r}=\left(\eta^{+}(r) \otimes \mathbb{C}\left[t, t^{-1}\right]\right) \oplus\left(\eta_{r}^{+} \otimes \mathbb{C}[t]\right) \oplus(\mathfrak{h} \otimes \mathbb{C}[t] t) \oplus\left(\eta_{r}^{-} \otimes \mathbb{C}[t] t\right) .
$$

Segue que $B_{r}=\bar{B}_{r} \oplus \widehat{\mathfrak{h}}$ é uma subálgebra de Borel de $\widehat{\mathfrak{g}}$ (para todo $0 \leq r \leq n$ ). Sendo assim, dado $\lambda \in \widehat{\mathfrak{h}}^{*}$, definimos

$$
M_{r}(\lambda)=U(\widehat{\mathfrak{g}}) \otimes_{U\left(B_{r}\right)} \mathbb{C} v_{\lambda}
$$


como o módulo do tipo Verma de $B_{r}$. Por outro lado, seja

$$
\rho_{r}=\mathfrak{a}_{r}+B_{r}+G
$$

uma subálgebra parabólica de $\widehat{\mathfrak{g}}$ onde

$$
\mathfrak{a}_{r}=\mathfrak{g}_{r} \otimes \mathbb{C}\left[t, t^{-1}\right] \oplus \mathbb{C} c
$$

e

$$
G=\underset{\substack{k \in \mathbb{Z} \\ k \neq 0}}{ } \widehat{\mathfrak{g}}_{k \delta} \oplus \mathbb{C} c
$$

Temos que

$$
\rho_{r}=\widehat{\mathfrak{a}}_{r} \oplus \mathfrak{N}_{r},
$$

onde $\widehat{\mathfrak{a}}_{r}=\mathfrak{a}_{r}+\widehat{\mathfrak{h}}$ e $\mathfrak{N}_{r}=\left(\eta^{+}(r) \otimes \mathbb{C}\left[t, t^{-1}\right]\right) \oplus(\mathfrak{h}(r) \otimes \mathbb{C}[t] t)$ (aqui $\mathfrak{h}(r)=\mathfrak{h}_{r}^{\perp}$ ). Note que $\left[\mathfrak{h}(r), \widehat{\mathfrak{a}}_{r}\right]=0$ e $\widehat{\mathfrak{a}}_{r}$ é um fator de Levi. Seja

$$
\widehat{M}_{r}(\widehat{\lambda})=U(\widehat{\mathfrak{g}}) \otimes_{U\left(\rho_{r}\right)} M\left(\lambda_{r}\right),
$$

onde $\lambda_{r}=\left.\lambda\right|_{\widehat{\mathfrak{h}}_{r}}, M\left(\lambda_{r}\right)$ é um módulo sobre $\widehat{\mathfrak{a}}_{r}$ e $\mathfrak{N}_{r} M\left(\lambda_{r}\right)=0$ (já que $\left[\mathfrak{h}(r), \widehat{\mathfrak{a}}_{r}\right]=0$ ). Usando a propriedade universal do produto tensorial, segue imediatamente que

$$
M_{r}(\lambda) \simeq \widehat{M}_{r}(\lambda)
$$

Agora podemos definir o conceito de $\mathbb{J}$-módulo do tipo Verma para $\mathbb{J} \subseteq \mathbb{N}^{*}$ fixado.

Definição 1.4.12. Considere $\rho_{r, \mathbb{J}}=\widehat{\mathfrak{a}}_{r} \oplus \mathfrak{N}_{r, \mathbb{I}}$ e $\mathfrak{N}_{r, \mathbb{J}}=\left(\eta^{+}(r) \otimes \mathbb{C}\left[t, t^{-1}\right]\right) \oplus \mathcal{K}_{\mathbb{J}}$, onde $\mathcal{K}_{\mathbb{J}}=\operatorname{span}_{\mathbb{C}}\left\{h \otimes t^{m} \mid \psi_{\mathbb{J}}(m)=1, h \in \mathfrak{h}(r)\right\}$. Temos que

$$
\widehat{M}_{r, \mathbb{J}}(\lambda)=U(\widehat{\mathfrak{g}}) \otimes_{U\left(\rho_{r, \mathbb{J}}\right)} M\left(\lambda_{r}\right)
$$

é o J- módulo do tipo Verma de $\rho_{r, \mathbb{J}}$.

\subsubsection{Primeira realização de campo livre}

Para obter uma realização de campo livre do módulo de Verma para $\widehat{\mathfrak{s l}}(2, \mathbb{C})$, Bernard e Felder utilizaram a construção de Borel-Weyl (ver [BF90]). Seja $\widehat{B}_{-}$um subgrupo de Borel do grupo de Loop $\widehat{S L}(2)$ correspondente a uma subálgebra de Borel $\mathfrak{b}_{\text {nat }}$. Então $\widehat{B}_{-}$ consiste de elementos

$$
\exp \left(\sum_{n \in \mathbb{Z}} x_{n} e_{n}\right) \exp \left(\sum_{m>0} y_{m} h_{m}\right),
$$

onde $x_{n}, y_{m}$ são funções coordenadas. Considere uma representação unidimensional $\chi: \widehat{B}_{-} \rightarrow \mathbb{C}$, onde $c$ age como escalar $K, h$ age como escalar $J$ (onde $J / 2$ é chamado de spin) e todos os elementos agem trivialmente. Então podemos construir um fibrado de linha sobre $\widehat{S L}(2) / \widehat{B}_{-}$, tomando o produto fibrado

$$
\mathcal{L}_{\chi}=\widehat{S L}(2) \times \widehat{B}_{-} \mathbb{C}
$$


e uma aplicação $g: \widehat{S L}(2) \times_{\widehat{B}_{-}} \mathbb{C} \rightarrow \widehat{S L}(2) / \widehat{B}_{-}$tal que $(x, z) \mapsto x \widehat{B}_{-}$. Diferenciando a ação do grupo $\widehat{S L}(2, \mathbb{C})$ que age nas seções do fibrado de linha, obtemos uma ação na álgebra de Lie $\mathfrak{g}$ e, assim, aplicando as duas anti-involuções

$$
e_{n} \leftrightarrow-f_{-n}, h_{n} \leftrightarrow h_{-n}, \quad c \leftrightarrow c
$$

$\mathrm{e}$

$$
x_{-n} \leftrightarrow \partial x_{n}, \quad y_{k} \leftrightarrow-\partial y_{k},
$$

obtemos a seguinte realização de campo livre de $\mathfrak{g}$ no espaço de Fock $\mathbb{C}\left[x_{m}, m \in \mathbb{Z}\right] \otimes$ $\mathbb{C}\left[y_{n}, n>0\right]:$

$f_{n} \mapsto x_{n}$,

$h_{n} \mapsto-2 \sum_{m \in \mathbb{Z}} x_{m+n} \partial x_{m}+\delta_{n<0} y_{-n}+\delta_{n>0} 2 n K \partial y_{n}+\delta_{n, 0} J$,

$e_{n} \mapsto-\sum_{m, k \in \mathbb{Z}} x_{k+m+n} \partial x_{k} \partial x_{m}+\sum_{k>0} y_{k} \partial x_{-k-n}+2 K \sum_{m>0} m \partial y_{m} \partial x_{m-n}+(K n+J) \partial x_{-n}$.

Este módulo é irredutível se, e somente se, $K \neq 0$. Se tomarmos $K=0$ e o quociente fora do submódulo gerado por $y_{m}, m>0$, obtemos a usualmente chamada primeira realização de campo livre de $\widehat{\mathfrak{s l}}(2, \mathbb{C})$. Este quociente é irredutível se, e somente se, $J \neq 0$ (ver [Fut94]). Estes resultados já foram generalizados em [Cox05] para todas as álgebras de Lie afim.

\subsubsection{Módulos de Wakimoto}

Aqui apresentamos a construção, para $\widehat{\mathfrak{s l}}(2, \mathbb{C})$, dos módulos de Wakimoto (ver [Wak86]) também conhecida como segunda realização de campos livres. Sejam

$$
a^{*}(z)=\sum_{m \in \mathbb{Z}} a_{m}^{*} z^{-m}, a(z)=\sum_{m \in \mathbb{Z}} a_{m} z^{-m-1} \text { e } b(z)=\sum_{m \in \mathbb{Z}} b_{m} z^{-m-1},
$$

onde $a_{n}=\left\{\begin{array}{ll}x_{n}, & \text { se } n<0 \\ \partial x_{n}, & \text { se } n \geq 0\end{array}, \quad a_{n}^{*}=\left\{\begin{array}{ll}x_{-n}, & \text { se } n \leq 0 \\ -\partial x_{-n}, & \text { se } n>0\end{array} \quad\right.\right.$ e $b_{m}=\left\{\begin{array}{ll}m \partial y_{m}, & \text { se } m \geq 0 \\ y_{-m}, & \text { se } m<0\end{array}\right.$. Temos que $\left[a_{n}, a_{m}^{*}\right]=\delta_{n+m, 0}$ e $\left[b_{n}, b_{m}\right]=n \delta_{n+m, 0}$.

Teorema 1.4.14. ([Wak86]). As fórmulas

$$
\begin{gathered}
c \mapsto K, \quad e(z) \mapsto a(z), \quad h(z) \mapsto-2: a^{*}(z) a(z):+b(z), \\
f(z) \mapsto-: a^{*}(z)^{2} a(z):+K \partial_{z} a^{*}(z)+a^{*}(z) b(z)
\end{gathered}
$$

definem a segunda realização de campos livres da álgebra de Lie afim $\mathfrak{s l}(2, \mathbb{C})$ agindo em

$$
\mathbb{C}\left[x_{n}, n \in \mathbb{Z}\right] \otimes \mathbb{C}\left[y_{m}, m>0\right] .
$$

Tais módulos são conhecidos como módulos de Wakimoto. Eles foram definidos para uma álgebra de Lie afim arbitrária por Feigin e Frenkel em [FF90] e [FF090].

Observação 1.4.15. Genericamente, os módulos de Wakimoto são isomorfos aos módulos de Verma. No caso dos módulos de Verma imaginários com os módulos de Wakimoto imaginários, este isomorfismo ocorre quando a carga central é não-nula. 


\subsubsection{Módulos intermediários de Wakimoto}

Consideramos dois casos extremos de subálgebras de Borel para álgebras de Lie afins: standard e natural. Mas se o grau de $\mathfrak{g}$ é maior que 1 então a correspondente álgebra de Lie afim possue outras classes de conjugação de subálgebras de Borel. É possível associar a cada subálgebra de Borel uma realização tipo bóson rearranjando os operadores de aniquilação e de criação, como foi feito na primeira e na segunda realização de campos livres. Para álgebras de Lie afins do tipo $A_{n}^{(1)}$ associadas à $\mathfrak{s l}(n+1, \mathbb{C})$ isto foi feito em [CF04], onde uma série de realizações tipo bóson foi construída dependendo de um parâmetro $0 \leq r \leq n$. Se $r=n$, estas construções coincidem com a construção de módulo de Wakimoto. Por outro lado, se $r=0$, a representação obtida nos dá uma realização no espaço de Fock descrita em [Cox05].

Seja $\mathfrak{b}_{r}$ a subálgebra de Borel de $\widetilde{\mathfrak{g}}$ correspondente à subálgebra parabólica de $\mathfrak{g}$ cuja parte semisimples do fator de Levi é $\mathfrak{g}_{r}$. Fixe $\widetilde{\lambda} \in \widetilde{\mathfrak{h}}^{*}$ e seja $M_{r}(\widetilde{\lambda})$ um módulo tipo Verma associado à $\mathfrak{b}_{r}$ e $\widetilde{\lambda}$. Quando $r=n$, este módulo é um módulo de Verma standard enquanto que no caso $r=0$ temos um módulo de Verma imaginário. Denote por $v_{\tilde{\lambda}}$ o gerador de $M_{r}(\widetilde{\lambda})$.

Seja $\widetilde{\lambda}_{r}=\left.\widetilde{\lambda}\right|_{\widetilde{\mathfrak{h}}_{r}}$. O módulo $M_{r}(\widetilde{\lambda})$ contém um $\widetilde{\mathfrak{g}}_{r}$-submódulo $M\left(\widetilde{\lambda}_{r}\right)=U\left(\widetilde{\mathfrak{g}}_{r}\right)\left(v_{\widetilde{\lambda}}\right)$ que é isomorfo ao módulo de Verma standard para $\widetilde{\mathfrak{g}}_{r}$. Se $\widetilde{\lambda}(c) \neq 0$ então a estrutura de submódulo $M_{r}(\widetilde{\lambda})$ é completamente determinada pela estrutura de submódulo de $M\left(\widetilde{\lambda}_{r}\right)$ (ver [Cox94] e [FS93]). No capítulo 3, define-se

$$
E_{i}(z)=\sum_{n \in \mathbb{Z}} E_{i n} z^{-n-1}, \quad F_{i}(z)=\sum_{n \in \mathbb{Z}} F_{i n} z^{-n-1}, \quad H_{i}(z)=\sum_{n \in \mathbb{Z}} H_{i n} z^{-n-1},
$$

e, para $a_{i j}(z)=\sum_{m \in \mathbb{Z}} a_{i, j, m} z^{-m-1}, a_{i j}^{*}(z)=\sum_{m \in \mathbb{Z}} a_{i, j, m}^{*} z^{-m}$ e $b_{i}(z)=\sum_{m \in \mathbb{Z}} b_{i, m} z^{-m-1}$, com $1 \leq i \leq n$, prova-se o seguinte teorema.

Teorema 1.4.16. ([CF04]). Seja $\lambda \in \mathfrak{H}^{*}$ e $\lambda_{i}=\lambda\left(H_{i}\right)$. As funções geradoras

$$
\begin{aligned}
F_{i}(z) \mapsto a_{i i} & +\sum_{j=i+1}^{n} a_{i j} a_{i+1, j}^{*}, \\
H_{i}(z) \mapsto 2: a_{i i} a_{i i}^{*}:+\sum_{j=1}^{i-1}\left(: a_{j i} a_{j i}^{*}:-: a_{j, i-1} a_{j, i-1}^{*}:\right) & \\
& +\sum_{j=i+1}^{n}\left(: a_{i j} a_{i j}^{*}:-: a_{i+1, j} a_{i+1, j}^{*}:\right)+b_{i}, \\
E_{i}(z) \mapsto: & a_{i i}^{*}\left(\sum_{k=1}^{i-1} a_{k, i-1} a_{k, i-1}^{*}-\sum_{k=1}^{i} a_{k i} a_{k i}^{*}\right):+\sum_{k=i+1}^{n} a_{i+1, k} a_{i k}^{*} \\
& -\sum_{k=1}^{i-1} a_{k, i-1} a_{k i}^{*}-a_{i i}^{*} b_{i}-\left(\delta_{i>r}(r+1)+\delta_{i \leq r}(i+1)-\gamma^{2}\right) \partial a_{i i}^{*}, \\
c \mapsto \gamma^{2}- & (r+1)
\end{aligned}
$$


definem uma representação no espaço de Fock $\mathbb{C}[\mathbf{x}] \otimes \mathbb{C}[\mathbf{y}]$. Acima, $a_{i j}$, $a_{i j}^{*}$ e $b_{i}$ denotam $a_{i j}(z), a_{i j}^{*}(z)$ e $b_{i}(z)$, respectivamente.

Esta realização tipo bóson de $\widetilde{s l}(n+1)$ depende do parâmetro $0 \leq r \leq n$ e define uma estrutura de módulo no espaço de Fock $\mathbb{C}[\mathbf{x}] \otimes \mathbb{C}[\mathbf{y}]$ que é chamada módulo intermediário de Wakimoto. Denote-o por $W_{n, r}(\lambda, \gamma)$ e considere um $\widetilde{\mathfrak{g}}_{r}$-submódulo $W=U\left(\widetilde{\mathfrak{g}}_{r}\right)\left(v_{\tilde{\lambda}}\right) \simeq W_{r, r}(\lambda, \gamma)$ de $W_{n, r}(\lambda, \gamma)$. Então $W$ é isomorfo ao módulo de Wakimoto $W_{\lambda(r), \widetilde{\gamma}}([\mathrm{FF} 090])$, onde $\lambda(r)=\left.\lambda\right|_{\mathfrak{h}_{r}}$ e $\widetilde{\gamma}=\gamma^{2}-(r+1)$. Já que módulos de Wakimoto são genericamente isomorfos aos módulos de Verma, os módulos intermediários de Wakimoto provam genericamente uma realização para módulos tipo Verma. 


\section{Capítulo 2}

\section{Os módulos de Verma d-imaginários}

O objetivo principal deste capítulo é apresentar ao leitor os resultados obtidos pelo autor em [Mar11]. Fixado um subconjunto $\mathbb{J} \subseteq \mathbb{N}^{*}$, construímos num certo espaço $\mathbb{C}[\mathbf{y}]$, (e posteriormente em $\mathbb{C}[\mathbf{x}, \mathbf{y}])$ operadores $b_{i m}$, com $m \in \mathbb{Z}$, e $1 \neq i \neq n$. Para nos auxiliar, definimos uma função $\psi_{\mathbb{J}}: \mathbb{Z}^{*} \rightarrow\{0,1\}$ e iniciamos nossa discussão sobre $\widehat{\mathfrak{s l}}(n, \mathbb{C})$ dividindo nossa análise para os casos $n=2$ e $n>2$. No caso $n=2$, apresentamos uma candidata $\varphi_{\mathbb{J}}: \widehat{\mathfrak{s l}}(2, \mathbb{C}) \rightarrow \mathfrak{g l}(\mathbb{C}[\mathbf{x}, \mathbf{y}])$ a qual satisfaz quase todas as relações necessárias e, então, a utilizamos para definir uma $\rho_{\mathbb{J}}$ com as propriedades desejadas. Já no caso $n>2$, apresentamos diretamente a candidata $\rho_{\mathbb{J}}: \widehat{\mathfrak{s l}}(n, \mathbb{C}) \rightarrow \mathfrak{g l}(\mathbb{C}[\mathbf{x}, \mathbf{y}])$ e, através da linguagem de distribuições formais, após enunciar e demonstrar alguns Lemas auxiliares, provamos as 6 relações necessárias obtendo, desta forma, uma realização para $\widehat{\mathfrak{s l}}(n, \mathbb{C})$. Isto é o que nos permite definir o conceito de módulo de Verma J-imaginário.

Seguindo este esquema, começamos os estudos referentes ao artigo [Mar11].

\subsection{Introdução}

Seja $\mathfrak{s l}(n, \mathbb{C})$ a álgebra de Lie complexa das $(n+1) \times(n+1)$-matrizes de traço nulo com forma de Killing $(X \mid Y)=\operatorname{tr}(X Y)$. Denotemos por $e_{i j}$ a matriz com 1 na $(i, j)$-ésima entrada e zero nas demais, com $i, j=1, \ldots, n$ e definimos $E_{i}=e_{i, i+1}, F_{i}=e_{i+1, i}$ e $H_{i}=e_{i i}-e_{i+1, i+1}$, onde $i=1, \ldots, n-1$. Então $E_{i}, F_{i}$, e $H_{i}$ 's formam um conjunto gerador de $\mathfrak{s l}(n, \mathbb{C})$.

A álgebra de Lie afim $\widehat{\mathfrak{s l}}(n, \mathbb{C})$ é a extensão central da álgebra de Loop $\mathfrak{s l}(n, \mathbb{C}) \otimes \mathbb{C}\left[t, t^{-1}\right]$ com centro unidimensional $\mathbb{C} c$. Para todo $X \in \mathfrak{s l}(n, \mathbb{C})$ e $m \in \mathbb{Z}$, seja $X_{m}=t^{m} \otimes X$. Então $\widehat{\mathfrak{s l}}(n, \mathbb{C})$ é gerado por $E_{i m}, F_{i m}$ e $H_{i m}$, com $m \in \mathbb{Z}$ e $1 \leqslant i \leqslant n$, e pelo elemento central $c$.

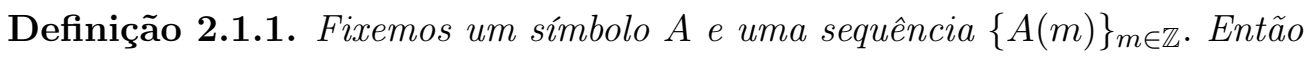

$$
\begin{gathered}
A^{+}(z)=\sum_{m \in \mathbb{Z}_{>0}} A(m) z^{-m}, \\
A^{-}(z)=\sum_{m \in \mathbb{Z}_{\leqslant 0}} A(m) z^{-m}, \\
A(z)=A^{+}(z)+A^{-}(z)=\sum_{m \in \mathbb{Z}} A(m) z^{-m},
\end{gathered}
$$




$$
\dot{A}(z)=\dot{A}^{+}(z)+\dot{A}^{-}(z)=\sum_{m \in \mathbb{Z}} m A(m) z^{-m}
$$

onde temos, por exemplo, que $\dot{H}_{i}^{+}(z)=\sum_{m \in \mathbb{Z}_{>0}} m H_{i m} z^{-m}$.

Teorema 2.1.6. São verdadeiras as seguintes relações em $\widehat{\mathfrak{s l}}(n, \mathbb{C})$.

$\left(R_{1}\right)\left[H_{i}(z), H_{j}(w)\right]=-\left(H_{i} \mid H_{j}\right) c \dot{\delta}(z / w)$,

$\left(R_{2}\right)\left[H_{i}(z), E_{j}(w)\right]=C_{i j} \sum_{n} E_{j}(z) z^{n} w^{-n}=C_{i j} E_{j}(z) \delta(z / w)$,

$\left(R_{3}\right)\left[H_{i}(z), F_{j}(w)\right]=-C_{i j} \sum_{n} F_{j}(z) z^{n} w^{-n}=-C_{i j} F_{j}(z) \delta(z / w)$,

$\left(R_{4}\right)\left[E_{i}(z), F_{j}(w)\right]=\delta_{i j}\left(H_{i}(z) \delta(z / w)-c \dot{\delta}(z / w)\right)$,

$\left(R_{5}\right)\left[E_{i}(z), E_{j}(w)\right]=\left[F_{i}(z), F_{j}(w)\right]=0$ se $C_{i j} \neq-1$,

$\left(R_{6}\right)\left[F_{i}\left(z_{1}\right), F_{i}\left(z_{2}\right), F_{j}(w)\right]=\left[E_{i}\left(z_{1}\right), E_{i}\left(z_{2}\right), E_{j}(w)\right]=0$ se $C_{i j}=-1$, onde $C=\left(C_{i j}\right)$ denota a matriz de Cartan do tipo $A_{n}$ e $[X, Y, Z]=[X,[Y, Z]]$.

Elementos $H_{i}, i=1, \ldots, n-1$, e $c$ geram uma subálgebra de Cartan de $\widehat{\mathfrak{s l}}(n, \mathbb{C})$ enquanto que $H_{i m}, m \in \mathbb{Z}, 1 \leqslant i \leqslant n$, e $c$ geram uma subálgebra de Heisenberg de $\widehat{\mathfrak{s l}}(n, \mathbb{C})$. O elemento central $c$ age como escalar no módulo irredutível $V$. Este escalar é chamado de carga central de $V$. Agora fixe $\gamma \in \mathbb{C}^{*}$ e para todo $1 \leqslant i \leqslant n$, fixe $\lambda_{i} \in \mathbb{C}$. Seja $2 c=\gamma^{2}$. Então defina

$$
\begin{gathered}
\mathbb{C}[\mathbf{x}]=\mathbb{C}\left[x_{i j, m} \mid i, j, m \in \mathbb{Z}, 1 \leqslant i, j \leqslant n\right] \\
\mathbb{C}[\mathbf{y}]=\mathbb{C}\left[y_{i m} \mid i, m \in \mathbb{N}^{*}, 1 \leqslant i \leqslant n\right]
\end{gathered}
$$

como sendo as álgebras geradas sobre $\mathbb{C}$ por $x_{i j, m}$ e $y_{i m}$, respectivamente. Em $\mathbb{C}[\mathbf{x}]$, defina os operadores

$$
\begin{aligned}
a_{i j, m} & =-x_{i j, m}, \\
a_{i j, m}^{*} & =\frac{\partial}{\partial x_{i j,-m}} .
\end{aligned}
$$

onde $\left[a_{i j, m}, a_{k l, p}^{*}\right]=\delta_{i k} \delta_{j l} \delta_{m,-p}$. Fixe um $\mathbb{J} \subseteq \mathbb{N}^{*}$ arbitrário. Em $\mathbb{C}[\mathbf{y}]$, defina operadores $b_{i m}$, com $m \in \mathbb{Z}$ e $1 \leqslant i \leqslant n$, da seguinte forma:

$$
b_{i m}= \begin{cases}-\gamma^{-1} \lambda_{i} & \text { se } m=0, \\ -\gamma m \frac{\partial}{\partial y_{i m}} & \text { se } m \in \mathbb{N}^{*} \backslash \mathbb{J}, \\ -\gamma^{-1} y_{i m} & \text { se } m \in \mathbb{J}, \\ \gamma m \frac{\partial}{\partial y_{i,-m}} & \text { se }-m \in \mathbb{J}, \\ -\gamma^{-1} y_{i,-m} & \text { se }-m \in \mathbb{N}^{*} \backslash \mathbb{J} .\end{cases}
$$

Temos que $\left[b_{i m}, b_{j p}\right]=m \delta_{i j} \delta_{m,-p}$.

Definição 2.1.12. Fixe $\mathbb{I} \subseteq \mathbb{N}^{*}$ e seja $\psi_{\mathbb{J}}: \mathbb{Z}^{*} \rightarrow\{0,1\}$ a função dada por

$$
\psi_{\mathbb{J}}(m)= \begin{cases}1 & \text { se } m \in \mathbb{J} \text { ou }-m \in \mathbb{N}^{*} \backslash \mathbb{J}, \\ 0 & \text { se } m \in \mathbb{N}^{*} \backslash \mathbb{J} \text { ou }-m \in \mathbb{J} .\end{cases}
$$


Seja $\mathfrak{g}=\widehat{\mathfrak{s l}}(n, \mathbb{C})$ e considere

$\mathrm{S}_{\psi_{\mathbb{J}}}=\left\{\alpha+m n \delta \mid \alpha \in \dot{\Delta}_{+}, m \in \mathbb{Z}\right\} \cup\left\{m \delta \mid m \in \mathbb{N}, \varphi_{\mathbb{J}}(m)=1\right\} \cup\left\{-m \delta \mid m \in \mathbb{N}, \varphi_{\mathbb{J}}(m)=0\right\}$.

Então os espaços

$$
\mathfrak{g}_{\psi_{\psi_{J}}}=\oplus_{\alpha \in \mathrm{S}_{\psi_{J}}} \mathfrak{g}_{\alpha} \text { e } \mathfrak{g}_{-\mathrm{S}_{\psi_{\mathbb{J}}}}=\oplus_{\alpha \in-\mathrm{S}_{\psi_{\mathbb{J}}}} \mathfrak{g}_{\alpha}
$$

são subálgebras de $\mathfrak{g}$ tais que

$$
\mathfrak{g}=\mathfrak{g}_{-\mathrm{S}_{\psi_{\mathbb{J}}}} \oplus \mathfrak{h} \oplus \mathfrak{g}_{\mathrm{S}_{\psi_{\mathbb{J}}}} .
$$

Seja $\lambda \in \mathfrak{h}^{*}$ e suponha que $\lambda(c)=a, a \in \mathbb{C}$. Seja $\mathfrak{b}_{\psi_{\mathbb{J}}}=\mathfrak{h} \oplus \mathfrak{g}_{\mathrm{S}_{\psi_{J}}}$ a subálgebra de Borel correspondente à $\mathrm{S}_{\psi_{J}}$, e observe que $\mathfrak{b}_{\psi_{\mathrm{J}}} \supset \mathbb{C} c \oplus \mathrm{L}_{\psi_{\mathrm{J}}}^{+}$. Seja $\mathbb{C} v_{\lambda}$ um módulo unidimensional de $\mathfrak{b}_{\psi_{\mathbb{J}}}$ tal que para todo $h \in \mathfrak{h}$, temos $\mathfrak{g}_{\mathrm{S}_{\psi_{\mathbb{J}}}} v_{\lambda}=0$ e $h v_{\lambda}=\lambda(h) v_{\lambda}$.

Definição 2.1.16. Defina o $\mathfrak{g}$-módulo

$$
\mathrm{M}_{\psi_{\mathbb{J}}}(\lambda)=\mathrm{M}_{\mathfrak{b}_{\psi_{J}}}(\lambda)=\mathrm{U}(\mathfrak{g}) \otimes \mathrm{U}\left(\mathfrak{b}_{\psi_{\mathbb{J}}}\right) \mathbb{C} v_{\lambda}
$$

como sendo o módulo de Verma J-imaginário. Identificando $1 \otimes v_{\lambda} \operatorname{com} v_{\lambda}, o \mathrm{U}(\mathrm{L})$ submódulo de $\mathrm{M}_{\psi_{\mathbb{J}}}(\lambda)$ gerado por $v_{\lambda}$ é isomorfo à $\mathrm{M}_{\psi_{\mathbb{J}}}(a)$. Se $\psi_{\mathbb{J}}(n)=1$ para todo $n$, então $\mathrm{M}_{\psi_{\mathrm{J}}}(\lambda)$ coincide com o módulo de Verma imaginário $\mathrm{M}_{\mathrm{S}}(\lambda)$, onde $\mathrm{S}=\Delta_{+}$.

Temos as seguintes propriedades básicas de $\mathrm{M}_{\psi_{\mathrm{J}}}(\lambda)$.

Proposição 2.1.18. [BBFK11, Proposição 3.4] Seja $\lambda \in \mathfrak{h}^{*}$ tal que $\lambda(c)=a$. Se $a \neq 0$, as seguintes afirmações são verdadeiras para $\mathrm{M}_{\psi_{\mathrm{J}}}(\lambda)$.

- $\mathrm{M}_{\psi_{\mathbb{J}}}(\lambda)$ é um $\mathrm{U}\left(\mathfrak{g}_{-\mathrm{S}_{\psi_{J}}}\right)$-módulo livre de posto 1 .

- $\mathrm{M}_{\psi_{\text {ग}}}(\lambda)$ tem um único submódulo maximal e assim um único quociente irredutível.

- $\operatorname{supp}\left(\mathrm{M}_{\psi_{J}}(\lambda)\right)=\bigcup_{\beta \in \dot{Q}_{+}}\{\lambda-\beta+n \delta \mid n \in \mathbb{Z}\}$ onde $\dot{\mathrm{Q}}_{+}$é um monóide abeliano livre gerado por todas as raízes simples em $\dot{\Delta}_{+}$.

- Se $\psi_{\mathbb{J}}(k) \neq \psi_{\mathbb{J}}(\ell)$ para algum $k, \ell \in \mathbb{N}$, então $\operatorname{dim} \mathrm{M}_{\psi_{\mathbb{J}}}(\lambda)_{\mu}=\infty$ para todo $\mu \in \operatorname{supp}\left(\mathrm{M}_{\psi_{\mathrm{J}}}(\lambda)\right)$.

Temos o seguinte critério de irredutibilidade para os módulos $\mathrm{M}_{\psi_{\mathrm{J}}}(\lambda)$.

Teorema 2.1.19. [BBFK11, Teorema 3.5] Seja $\lambda \in \mathfrak{h}^{*}, \lambda(c)=$ a. Então $\mathrm{M}_{\psi_{\mathrm{J}}}(\lambda)$ é irredutivel se, e somente se, $a \neq 0$.

Agora defina

$$
\mathbb{C}[\mathbf{x}, \mathbf{y}]=\mathbb{C}[\mathbf{x}] \otimes_{\mathbb{C}} \mathbb{C}[\mathbf{y}]
$$




\subsection{Realização de campos livres de $\widehat{\mathfrak{s l}}(n, \mathbb{C})$}

\subsubsection{O caso $n=2$}

Aqui denotaremos $E_{m}$ ao invés de $E_{1 m}, a_{m}$ ao invés de $a_{11, m}$, etc.

Definição 2.2.1. Seja $\varphi_{\mathbb{J}}: \widehat{\mathfrak{s l}}(2, \mathbb{C}) \rightarrow \mathfrak{g l}(\mathbb{C}[\mathbf{x}, \mathbf{y}])$ a função dada por $\varphi_{\mathbb{J}}\left(E_{m}\right)=-\frac{\partial}{\partial x_{m}}$, $\varphi_{\mathbb{J}}\left(F_{m}\right)=\sum_{l, j} x_{l} x_{j-l-m} \frac{\partial}{\partial x_{j}}+\sum_{j \in \mathbb{J}} x_{j-m} \frac{\partial}{\partial y_{j}}+2 K \sum_{i \in \mathbb{J}} j y_{j} x_{-j-m}+K m x_{-m}-2 J x_{-m}$, $\varphi_{\mathbb{J}}\left(H_{m}\right)=-2 \sum_{j} x_{j-m} \frac{\partial}{\partial x_{j}}-\psi_{\mathbb{J}}(m) \frac{\partial}{\partial y_{m}}+\psi_{\mathbb{J}}(-m) 2 m K y_{-m}+2 \delta_{m, 0} J$.

Como estamos analisando o caso $n=2$, é suficiente verificarmos as relações $\left(R_{1}\right)-\left(R_{4}\right)$, as quais seguem diretamente do Lema abaixo.

Lema 2.2.2. Temos as relações

$(a)\left[\varphi_{\mathbb{J}}\left(H_{m}\right), \varphi_{\mathbb{J}}\left(E_{n}\right)\right]=2 \varphi_{\mathbb{J}}\left(E_{m+n}\right)$,

(b) $\left[\varphi_{\mathbb{J}}\left(H_{m}\right), \varphi_{\mathbb{J}}\left(H_{n}\right)\right]=2 \delta_{m,-n} m K$,

(c) $\left[\varphi_{\mathbb{J}}\left(H_{m}\right), \varphi_{\mathbb{J}}\left(F_{n}\right)\right]=-2 \varphi_{\mathbb{J}}\left(F_{m+n}\right)$,

$(d)\left[\varphi_{\mathbb{J}}\left(E_{m}\right), \varphi_{\mathbb{J}}\left(F_{n}\right)\right]=\varphi_{\mathbb{J}}\left(H_{m+n}\right)+m \delta_{m,-n} K$.

Demonstração. Temos

$$
\begin{aligned}
(a)\left[\varphi_{\mathbb{J}}\left(H_{m}\right), \varphi_{\mathbb{J}}\left(E_{n}\right)\right]= & {\left[-2 \sum_{j} x_{j-m} \frac{\partial}{\partial x_{j}}-\psi_{\mathbb{J}}(m) \frac{\partial}{\partial y_{m}}\right.} \\
& \left.+\psi_{\mathbb{J}}(-m) 2 m K y_{-m}+2 \delta_{m, 0} J,-\frac{\partial}{\partial x_{n}}\right] \\
= & {\left[-2 \sum_{j} x_{j-m} \frac{\partial}{\partial x_{j}},-\frac{\partial}{\partial x_{n}}\right] } \\
= & 2 \frac{\partial}{\partial x_{m+n}} \\
= & 2 \varphi_{\mathbb{J}}\left(E_{m+n}\right) .
\end{aligned}
$$


(b) $\left[\varphi_{\mathbb{J}}\left(H_{m}\right), \varphi_{\mathbb{J}}\left(H_{n}\right)\right]=\left[-2 \sum_{j} x_{j-m} \frac{\partial}{\partial x_{j}}-\psi_{\mathbb{J}}(m) \frac{\partial}{\partial y_{m}}+\psi_{\mathbb{J}}(-m) 2 m K y_{-m}+2 \delta_{m, 0} J\right.$,

$$
\begin{aligned}
& \left.-2 \sum_{j} x_{j-n} \frac{\partial}{\partial x_{j}}-\psi_{\mathbb{J}}(n) \frac{\partial}{\partial y_{n}}+\psi_{\mathbb{J}}(-n) 2 n K y_{-n}+2 \delta_{n, 0} J\right] \\
= & 4 \sum_{j} x_{j-m} \frac{\partial}{\partial x_{j+n}}-4 \sum_{j} x_{j-n} \frac{\partial}{\partial x_{j+m}} \\
& -\delta_{m,-n} \psi_{\mathbb{J}}(m) \psi_{\mathbb{J}}(-n) 2 n K \\
& +2 \delta_{m,-n} \psi_{\mathbb{J}}(n) \psi_{\mathbb{J}}(-m) K m+0 \\
= & 2 \delta_{m,-n} m K\left(\psi_{\mathbb{J}}(m)+\psi_{\mathbb{J}}(-m)\right) \\
= & 2 \delta_{m,-n} m K .
\end{aligned}
$$

(c) $\left[\varphi_{\mathbb{J}}\left(H_{m}\right), \varphi_{\mathbb{J}}\left(F_{n}\right)\right]=\left[-2 \sum_{j} x_{j-m} \frac{\partial}{\partial x_{j}}-\psi_{\mathbb{J}}(m) \frac{\partial}{\partial y_{m}}+\psi_{\mathbb{J}}(-m) 2 m K y_{-m}\right.$

$$
\begin{aligned}
& +2 \delta_{m, 0} J, \sum_{l, i} x_{l} x_{i-l-n} \frac{\partial}{\partial x_{i}}+\sum_{i \in \mathbb{J}} x_{i-n} \frac{\partial}{\partial y_{i}} \\
& \left.+2 K \sum_{i \in \mathbb{J}} i y_{i} x_{-i-n}+K n x_{-n}-2 J x_{-n}\right] \\
= & -2\left(\sum_{j} x_{j-m}\left(\sum_{i} x_{i-j-n} \frac{\partial}{\partial x_{i}}+\sum_{l} x_{l} \frac{\partial}{\partial x_{j+l+n}}\right)\right. \\
& \left.-\sum_{i, l} x_{l} x_{i-l-n} \frac{\partial}{\partial x_{i+m}}\right)-2 \sum_{j} x_{j-m} \psi_{\mathbb{J}}(j+n) \frac{\partial}{\partial y_{j+n}} \\
& -4 K \sum_{j} x_{j-m} \psi_{\mathbb{J}}(-j-n)(-j-n) y_{-j-n}-2 K n x_{-n-m} \\
& +4 J X_{-n-m}-2 \psi_{\mathbb{J}}(m) K \psi_{\mathbb{J}}(m) m x_{-m-n} \\
& -\psi_{\mathbb{J}}(-m) 2 K m \psi_{\mathbb{J}}(-m) x_{-m-n} \\
= & -4 \sum_{j, i} x_{j} x_{i-j-m-n} \frac{\partial}{\partial x_{i}}+2 \sum_{l, i} x_{l} x_{i-l-m-n} \frac{\partial}{\partial x_{i}} \\
& -2 \sum_{j} \psi_{\mathbb{J}}(j) x_{j-m-n} \frac{\partial}{\partial y_{j}}-4 K \sum_{j} \psi_{\mathbb{J}}(-j)(-j) y_{-j} x_{j-m-n} \\
& -2 K n x_{-m-n}+4 J x_{-m-n}-2 K m x_{-m-n} \\
= & -2 \sum_{j, i} x_{j} x_{i-j-m-n} \frac{\partial}{\partial x_{i}}-2 \sum_{j \in \mathbb{J}} x_{j-m-n} \frac{\partial}{\partial x_{j}} \\
& -4 K \sum_{j \in \mathbb{J}} j y_{j} x_{-j-m-n}-2 K(m+n) x_{-m-n}+4 J x_{-m-n} \\
= & -2 \varphi_{\mathbb{J}}\left(F_{m+n}\right) .
\end{aligned}
$$


$(d)\left[\varphi_{\mathbb{J}}\left(E_{m}\right), \varphi_{\mathbb{J}}\left(F_{n}\right)\right]=\left[-\frac{\partial}{\partial x_{m}}, \sum_{l, j} x_{l} x_{j-l-n} \frac{\partial}{\partial x_{j}}+\sum_{j \in \mathbb{J}} x_{j-n} \frac{\partial}{\partial y_{j}}\right.$

$$
\begin{aligned}
& \left.+2 K \sum_{j \in \mathbb{J}} j y_{j} x_{-j-n}+K n x_{-n}-2 J x_{-n}\right] \\
=- & \left(\sum_{j} x_{j-m-n} \frac{\partial}{\partial x_{j}}+\sum_{l} x_{l} \frac{\partial}{\partial x_{l+m+n}}+\psi_{\mathbb{J}}(m+n) \frac{\partial}{\partial y_{m+n}}\right. \\
& \left.-2 K \psi_{\mathbb{J}}(-m-n)(m+n) Y_{-m-n}-\delta_{m,-n}(K m+2 J)\right) \\
= & -2 \sum_{j} X_{j-m-n} \frac{\partial}{\partial x_{j}}-\psi_{\mathbb{J}}(m+n) \frac{\partial}{\partial y_{m+n}} \\
& +2 \psi_{\mathbb{J}}(-m-n)(m+n) K y_{-m-n}+2 \delta_{m,-n} J+K m \delta_{m,-n} \\
= & \varphi_{\mathbb{J}}\left(H_{m+n}\right)+m \delta_{m,-n} K .
\end{aligned}
$$

Observe que, infelizmente,

$$
\begin{aligned}
\varphi_{\mathbb{J}}\left(F_{m}\right) \cdot 1= & \left(\sum_{l, j} x_{l} x_{j-l-m} \frac{\partial}{\partial x_{j}}+\sum_{j \in \mathbb{J}} x_{j-m} \frac{\partial}{\partial y_{j}}\right. \\
& \left.+2 K \sum_{i \in \mathbb{J}} j y_{j} x_{-j-m}+K m x_{-m}-2 J x_{-m}\right) \cdot 1 \\
=+ & 2 K \sum_{i \in \mathbb{J}} j y_{j} x_{-j-m}+K m x_{-m}-2 J x_{-m},
\end{aligned}
$$

que é uma soma infinita se o conjunto $\mathbb{J} \subseteq \mathbb{N}^{*}$ for infinito, $\log 0 \varphi_{\mathbb{J}}$ não é a função desejada pois $\varphi_{\mathbb{J}}\left(F_{m}\right)$ sequer está bem-definida. Para solucionar este problema, seja $\rho_{1}: \widehat{\mathfrak{s l}}(2, \mathbb{C}) \rightarrow \widehat{\mathfrak{s l}}(2, \mathbb{C})$ a função dada por

$$
\rho_{1}\left(E_{m}\right)=-F_{-m}, \rho_{1}\left(F_{m}\right)=-E_{-m}, \rho_{1}\left(H_{m}\right)=H_{-m} \text { e } \rho_{1}(K)=K
$$

e seja $\rho_{2}: \mathfrak{g l}(\mathbb{C}[\mathbf{x}, \mathbf{y}]) \rightarrow \mathfrak{g l}(\mathbb{C}[\mathbf{x}, \mathbf{y}])$ a função dada por

$$
\rho_{2}\left(x_{-m}\right)=\frac{\partial}{\partial x_{m}}, \quad \rho_{2}\left(\frac{\partial}{\partial x_{m}}\right)=x_{-m}, \quad \rho_{2}\left(y_{k}\right)=-\frac{\partial}{\partial y_{k}} \text { e } \rho_{2}\left(\frac{\partial}{\partial y_{k}}\right)=-y_{k} .
$$

Observe que $\rho_{1}$ e $\rho_{2}$ são antiautomorfismos de $\widehat{\mathfrak{s l}}(2, \mathbb{C})$ e $\mathfrak{g l}(\mathbb{C}[\mathbf{x}, \mathbf{y}])$, respectivamente.

Definição 2.2.5. Seja $\rho_{\mathbb{J}}=\rho_{2} \circ \varphi_{\mathbb{J}} \circ \rho_{1}$. Temos

(a) $\rho_{\mathbb{J}}\left(F_{m}\right)=\rho_{2} \circ \varphi_{\mathbb{J}} \circ \rho_{1}\left(F_{m}\right)=\rho_{2} \circ \varphi_{\mathbb{J}}\left(-E_{-m}\right)=\rho_{2}\left(\frac{\partial}{\partial x_{-m}}\right)=x_{m}$. 
(b) $\rho_{\mathbb{J}}\left(E_{m}\right)=\rho_{2} \circ \varphi_{\mathbb{J}} \circ \rho_{1}\left(E_{m}\right)$

$$
\begin{aligned}
&=\rho_{2} \circ \varphi_{\mathbb{J}}\left(-F_{-m}\right) \\
&=\rho_{2}\left(-\sum_{l, j} x_{l} x_{j-l+m} \frac{\partial}{\partial x_{j}}-\sum_{j \in \mathbb{J}} x_{j+m} \frac{\partial}{\partial y_{j}}\right. \\
&\left.-2 K \sum_{j \in \mathbb{J}} j y_{j} x_{-j+m}-K(-m) x_{m}+2 J x_{m}\right) \\
&=- \sum_{l, j} x_{-j} \frac{\partial}{\partial x_{-l}} \frac{\partial}{\partial x_{-j+l-m}}-\sum_{j \in \mathbb{J}}\left(-y_{j}\right) \frac{\partial}{\partial x_{-j-m}} \\
&-2 K \sum_{j \in \mathbb{J}} j\left(-\frac{\partial}{\partial y_{j}}\right) \frac{\partial}{\partial x_{j-m}} \frac{\partial}{\partial x_{-m}}+2 J \frac{\partial}{\partial x_{-m}} \\
&=- \sum_{l, j} x_{j} \frac{\partial}{\partial x_{l}} \frac{\partial}{\partial x_{j-l-m}}+\sum_{j \in \mathbb{J}} y_{j} \frac{\partial}{\partial x_{-j-m}} \\
&+2 K \sum_{j \in \mathbb{J}} j \frac{\partial}{\partial y_{j}} \frac{\partial}{\partial x_{j-m}}+(K m+2 J) \frac{\partial}{\partial x_{-m}} \\
&=- \sum_{l, j} x_{j+l+m} \frac{\partial}{\partial x_{l}} \frac{\partial}{\partial x_{j}}+\sum_{j \in \mathbb{J}} y_{j} \frac{\partial}{\partial x_{-j-m}} \\
&+2 K \sum_{j \in \mathbb{J}} j \frac{\partial}{\partial y_{j}} \frac{\partial}{\partial x_{j-m}}+(K m+2 J) \frac{\partial}{\partial x_{-m}} .
\end{aligned}
$$

(c) $\rho_{\mathbb{J}}\left(H_{m}\right)=\rho_{2} \circ \varphi_{\mathbb{J}} \circ \rho_{1}\left(H_{m}\right)$

$$
\begin{aligned}
& =\rho_{2} \circ \varphi_{\mathbb{J}}\left(H_{-m}\right) \\
& =\rho_{2}\left(-2 \sum_{j} x_{j+m} \frac{\partial}{\partial x_{j}}-\psi_{\mathbb{J}}(-m) \frac{\partial}{\partial y_{-m}}+\psi_{\mathbb{J}}(m) 2(-m) K y_{m}+2 \delta_{m, 0} J\right) \\
& =-2 \sum_{j} x_{-j} \frac{\partial}{\partial x_{-j-m}}+\psi_{\mathbb{J}}(-m) y_{-m}+\psi_{\mathbb{J}}(m) 2 m K \frac{\partial}{\partial y_{m}}+2 \delta_{m, 0} J \\
& =-2 \sum_{j} x_{j+m} \frac{\partial}{\partial x_{j}}+\psi_{\mathbb{J}}(-m) y_{-m}+\psi_{\mathbb{J}}(m) 2 m K \frac{\partial}{\partial y_{m}}+2 \delta_{m, 0} J .
\end{aligned}
$$

A função $\rho_{\mathbb{J}}$ satisfaz as relações de $\left(R_{1}\right)$ à $\left(R_{4}\right)$, já que $\varphi_{\mathbb{J}}$ as satisfazem e $\rho_{1}$ e $\rho_{2}$ são antiautomorfismos. Desta forma, temos uma representação para $\widehat{\mathfrak{s l}}(2, \mathbb{C})$ com as propriedades desejadas.

\subsubsection{O caso $n>2$}

Definição 2.2.6. Seja $\rho_{\mathbb{J}}$ a função dada por 


$$
\begin{aligned}
\rho_{\mathbb{J}}\left(E_{r m}\right)= & \sum_{l_{1}, l_{2} \in \mathbb{Z}} a_{r, r+1, m-l_{1}-l_{2}} a_{r, r+1, l_{1}}^{*} a_{r, r+1, l_{2}}^{*}+\sum_{l \in \mathbb{Z}}\left(-\sum_{j=r+2}^{n} a_{r+1, j, m-l} a_{r+1, j, l}^{*}\right) \\
& +\sum_{l_{1}, l_{2} \in \mathbb{Z}} a_{r, j, m-l_{1}-l_{2}} a_{r, j, l_{1}}^{*} a_{r, j, l_{2}}^{*}-\sum_{l_{1}, l_{2} \in \mathbb{Z}} a_{r+1, j, m-l_{1}-l_{2}} a_{r+1, j, l_{1}}^{*} a_{r, r+1, l_{2}}^{*} \\
& -\gamma \sum_{l \in \mathbb{Z}}\left(a_{r, r+1, l}^{*} b_{r, m-l}+\frac{1}{2} \delta_{m<0}\left(a_{r, r+1, l}^{*} b_{r-1, m-l}+a_{r, r+1, l}^{*} b_{r+1, m-l}\right)\right) \\
& -\frac{\gamma}{2} a_{r, r+1, m+1}^{*}, \\
\rho_{\mathbb{J}}\left(F_{r m}\right)= & a_{r, r+1, m}+\sum_{j=1}^{r-1} \sum_{l \in \mathbb{Z}} a_{j, r+1, m-l} a_{j, r, l}^{*}, \\
\rho_{\mathbb{J}}\left(H_{r m}\right)= & \sum_{l \in \mathbb{Z}}\left(2 a_{r, r+1, m-l} a_{r, r+1, l}^{*}+\sum_{i=1}^{r-1} a_{i, r+1, m-l} a_{i, r+1, l}^{*}\right. \\
& -\sum_{i=1}^{r-1} a_{i, r, m-l} a_{i, r, l}^{*}+\sum_{j=r+2}^{n} a_{r, j, m-l} a_{r, j, l}^{*}-\sum_{j=r+2}^{n} a_{r+1, j, m-l} a_{r+1, j, n}^{*} \\
& \left.-\gamma b_{r m}+\delta_{m<0} \frac{\gamma}{2}\left(b_{r-1, m}+b_{r+1, m}\right)\right) . \\
& ,
\end{aligned}
$$

Teorema 2.2.7. A função $\rho_{\mathbb{J}}: \widehat{\mathfrak{s l}}(n, \mathbb{C}) \rightarrow \mathfrak{g l}(\mathbb{C}[\mathbf{x}, \mathbf{y}])$ é uma realização de $\widehat{\mathfrak{s l}}(n, \mathbb{C})$.

Observação 2.2.8. Para provar que $\rho_{\mathbb{J}}$ define uma representação de $\widehat{\mathfrak{s l}}(n, \mathbb{C}), \quad a$ reescrevemos na linguagem de distribuições formais. Assim para provar o Teorema 2.2.7, basta mostrar que para

$$
\begin{aligned}
\rho_{\mathbb{J}}\left(F_{r}\right)(w)= & -a_{r, r+1}(w)+\sum_{j=1}^{r-1} a_{j, r+1}(w) a_{j r}^{*}(w), \\
\rho_{\mathbb{J}}\left(H_{r}\right)(w)= & 2 a_{r, r+1}(w) a_{r, r+1}^{*}(w)+\sum_{i=1}^{r-1}\left(a_{i, r+1}(w) a_{i, r+1}^{*}(w)-a_{i r}(w) a_{i r}^{*}(w)\right) \\
& +\sum_{j=r+2}^{n}\left(a_{r j}(w) a_{r j}^{*}(w)-a_{r+1, j}(w) a_{r+1, j}^{*}(w)\right) \\
& -\gamma b_{r}(w)+\frac{\gamma}{2}\left(b_{r-1}^{+}(w)+b_{r+1}^{+}(w)\right), \\
\rho_{\mathbb{J}}\left(E_{r}\right)(w)= & a_{r, r+1}(w) a_{r, r+1}^{*}(w)^{2}-\sum_{j=r+2}^{n} a_{r+1, j}(w) a_{r+1, j}^{*}(w)+\sum_{j=1}^{r-1} a_{j r}(w) a_{j, r+1}^{*}(w) \\
& +\sum_{j=r+2}^{n}\left(a_{r j}(w) a_{r j}^{*}(w)-a_{r+1, j}(w) a_{r+1, j}^{*}(w)\right) a_{r, r+1}^{*}(w) \\
& -\gamma a_{r, r+1}^{*}(w) b_{r}(w)+\frac{\gamma}{2} a_{r, r+1}^{*}(w)\left(b_{r-1}^{+}(w)+b_{r+1}^{+}(w)\right)-\frac{\gamma}{2} a_{r, r+1}^{*}(w),
\end{aligned}
$$

as relações $\left(R_{1}\right)-\left(R_{6}\right)$ são verdadeiras.

Para provar o teorema, precisamos dos Lemas à seguir.

Lema 2.2.9. Temos as seguintes relações 
(a) $\left[a_{i j}(z), a_{k l}^{*}(w)\right]=\delta_{i, k} \delta_{j, l} \delta(z / w)$,

(b) $\left[a_{i j}(z) a_{k l}^{*}(z), a_{m n}(w) a_{p q}^{*}(w)\right]$

$$
=\delta_{p i} \delta_{q j} a_{m n}(w) a_{k l}^{*}(z) \delta(z / w)-\delta_{k m} \delta_{l n} a_{i j}(z) a_{p q}^{*}(w) \delta(w / z),
$$

(c) $\left[a_{i j}(z), \dot{a}_{k l}^{*}(w)\right]=\delta_{i k} \delta_{j l} \dot{\delta}(z / w)$,

(d) $\dot{a}_{i j}^{*}(z) \delta(w / z)=a_{i j}^{*}(z) \dot{\delta}(w / z)-a_{i j}^{*}(w) \dot{\delta}(w / z)$,

(e) $\left[b_{i}(z), b_{j}^{ \pm}(w)\right]=\left[b_{i}^{\mp}(z), b_{j}^{ \pm}(w)\right]=\delta_{i j} \dot{\delta}^{\mp}(w / z)$,

$(f)\left[b_{i}(z), b_{j}(w)\right]=\left[b_{i}^{+}(z), b_{j}^{-}(w)\right]+\left[b_{i}^{-}(z), b_{j}^{+}(w)\right]=\delta_{i j} \dot{\delta}(w / z)$,

(g) $\left[a_{r, r+1}(z) a_{r, r+1}^{*}(z), a_{r, r+1}(w) a_{r, r+1}^{*}(w) a_{r, r+1}^{*}(w)\right]$

$$
=a_{r, r+1}(w) a_{r, r+1}^{*}(z) a_{r, r+1}^{*}(w) \delta(w / z),
$$

(h) $\left[a_{r, j}(z) a_{r j}^{*}(z), f(w) a_{k l}^{*}(w)\right]=f(w) \delta_{r, k} \delta_{j l} a_{r j}^{*}(z) \delta(w / z)$,

onde $f(w)$ comuta com $a_{r j}(z), a_{r j}^{*}(z)$ e $a_{k l}^{*}(w)$.

Demonstração. Temos

(a) $\left[a_{i j}(z), a_{k l}^{*}(w)\right]=\sum_{m, n \in \mathbb{Z}}\left[a_{i j}(m), a_{k l}^{*}(n)\right] z^{-m} w^{-n}=\sum_{m, n \in \mathbb{Z}} \delta_{i k} \delta_{j l} \delta_{m,-n} z^{-m} w^{-n}$

$$
=\sum_{n \in \mathbb{Z}} \delta_{i k} \delta_{j l} z^{n} w^{-n}=\delta_{i k} \delta_{j l} \delta(z / w) .
$$

(b) $\left[a_{i j}(z) a_{k l}^{*}(z), a_{m n}(w) a_{p q}^{*}(w)\right]=a_{i j}(z)\left[a_{k l}^{*}(z), a_{m n}(w)\right] a_{p q}^{*}(w)$

$$
\begin{aligned}
& +a_{i j}(z) a_{m n}(w) a_{k l}^{*}(z) a_{p q}^{*}(w)-a_{m n}(w)\left[a_{p q}^{*}(w), a_{i j}(z)\right] a_{k l}^{*}(z)-a_{m n}(w) a_{i j}(z) a_{p q}^{*}(w) a_{k l}^{*}(z) \\
= & \delta_{p i} \delta_{q j} a_{m n}(w) a_{k l}^{*}(z) \delta(z / w)-\delta_{k m} \delta_{l n} a_{i j}(z) a_{p q}^{*}(w) \delta(w / z) .
\end{aligned}
$$

(c) $\left[a_{i j}(z), \dot{a}_{k l}^{*}(w)\right]=\sum_{m, n \in \mathbb{Z}}\left[a_{i j}(m), n a_{k l}^{*}(n)\right] z^{-m} w^{-n}=\sum_{m, n \in \mathbb{Z}} n \delta_{i k} \delta_{j l} \delta_{m,-n} z^{-m} w^{-n}$

$$
=\sum_{n \in \mathbb{Z}} \delta_{i k} \delta_{j l} n z^{n} w^{-n}=\delta_{i k} \delta_{j l} \dot{\delta}(z / w)
$$

(d) $\dot{a}_{i j}^{*}(z) \delta(w / z)=\left(\sum_{m \in \mathbb{Z}}(-m) a_{i j}^{*}(m) z^{-m}\right)\left(\sum_{n \in \mathbb{Z}} z^{-n} w^{n}\right)=\sum_{m, n \in \mathbb{Z}}(-m) a_{i j}^{*}(m) z^{-m-n} w^{n}$

$$
\begin{aligned}
& =\sum_{m, n \in \mathbb{Z}}-(m+n) a_{i j}^{*}(m) z^{-m-n} w^{(m+n)-m}+\sum_{m, n \in \mathbb{Z}} n a_{i j}^{*}(m) z^{-m-n} w^{n} \\
& =\sum_{m, n \in \mathbb{Z}}-n a_{i j}^{*}(m) z^{-n} w^{n-m}+\sum_{m, n \in \mathbb{Z}} n a_{i j}^{*}(m) z^{-m} w^{n} z^{-n} \\
& =-\left(\sum_{m \in \mathbb{Z}} a_{i j}^{*}(m) w^{-m}\right) \dot{\delta}(w / z)+\sum_{m \in \mathbb{Z}} a_{i j}^{*}(m) z^{-m} \dot{\delta}(w / z) \\
& =a_{i j}^{*}(z) \dot{\delta}(w / z)-a_{i j}^{*}(w) \dot{\delta}(w / z) .
\end{aligned}
$$


(e) $\left[b_{i}(z), b_{j}^{-}(w)\right]=\sum_{\substack{m \in \mathbb{Z} \\ n \in \mathbb{Z}_{-}^{*}}}\left[b_{i m}, b_{j}(n)\right] z^{-m} w^{-n}=\sum_{\substack{m \in \mathbb{Z} \\ n \in \mathbb{Z}_{-}^{*}}} m \delta_{i j} \delta_{m,-n} z^{-m} w^{-n}$

$$
\begin{aligned}
& =\sum_{\substack{m \in \mathbb{Z}_{+}^{*} \\
n \in \mathbb{Z}_{-}^{*}}} m \delta_{i j} \delta_{m,-n} z^{-m} w^{-n}=\left[b_{i}^{+}(z), b_{j}^{-}(w)\right]=\sum_{n \in \mathbb{Z}_{-}}(-n) \delta_{i j} z^{n} w^{-n} \\
& =\delta_{i j} \dot{\delta}^{+}(w-z)
\end{aligned}
$$

e analogamente para a outra expressão. Juntando as duas, temos

$$
\begin{aligned}
(f)\left[b_{i}(z), b_{j}(w)\right] & =\left[b_{i}^{+}(z), b_{j}^{-}(w)\right]+\left[b_{i}^{-}(z), b_{j}^{+}(w)\right]=\sum_{m, n \in \mathbb{Z}} m \delta_{i j} \delta_{m,-n} z^{-m} w^{-n} \\
& =\delta_{i j} \sum_{n \in \mathbb{Z}}(-n) z^{n} w^{-n}=\delta_{i j} \dot{\delta}(w / z) .
\end{aligned}
$$

Além disso,

(g) $\left[a_{r, r+1}(z) a_{r, r+1}^{*}(z), a_{r, r+1}(w) a_{r, r+1}^{*}(w) a_{r, r+1}^{*}(w)\right]$

$$
\begin{aligned}
= & a_{r, r+1}(z)\left[a_{r, r+1}^{*}(z), a_{r, r+1}(w)\right] a_{r, r+1}^{*}(w) a_{r, r+1}^{*}(w) \\
& -a_{r, r+1}(w) a_{r, r+1}^{*}(w)\left[a_{r, r+1}^{*}(w), a_{r, r+1}(z)\right] a_{r, r+1}^{*}(z) \\
& +a_{r, r+1}(z) a_{r, r+1}(w) a_{r, r+1}^{*}(z) a_{r, r+1}^{*}(w) a_{r, r+1}^{*}(w) \\
& -a_{r, r+1}(w) a_{r, r+1}^{*}(w) a_{r, r+1}(z) a_{r, r+1}^{*}(w) a_{r, r+1}^{*}(z) \\
= & -a_{r, r+1}(z) \delta(w / z) a_{r, r+1}^{*}(w) a_{r, r+1}^{*}(w) \\
& +a_{r, r+1}(w) a_{r, r+1}^{*}(w) \delta(w / z) a_{r, r+1}^{*}(z) \\
& +a_{r, r+1}(w)\left[a_{r, r+1}(z), a_{r, r+1}^{*}(w)\right] a_{r, r+1}^{*}(z) a_{r, r+1}^{*}(w) \\
= & a_{r, r+1}(w) a_{r, r+1}^{*}(z) a_{r, r+1}^{*}(w) \delta(w / z) .
\end{aligned}
$$

(h) $\left[a_{r j}(z) a_{r j}^{*}(z), f(w) a_{k l}^{*}(w)\right]=f(w)\left(a_{r j}(z) a_{r j}^{*}(z) a_{k l}^{*}(w)-a_{k l}^{*}(w) a_{r j}(z) a_{r j}^{*}(z)\right)$

o que prova o Lema.

$$
=f(w)\left[a_{r j}(z), a_{k l}^{*}(w)\right] a_{r j}^{*}(z)=f(w) \delta_{r, k} \delta_{j l} a_{r j}^{*}(z) \delta(w / z),
$$

Lema 2.2.10. São verdadeiras

(i) $\sum_{j=r+2}^{n} \sum_{k=1}^{s-1}\left[a_{k s}(w) a_{k, s+1}^{*}(w), a_{r j}(z) a_{r j}^{*}(z)\right]=-\delta_{s, r+1} a_{r, r+1}(w) a_{r, r+2}^{*}(z) \delta(w / z)$,

(j) $\sum_{j=1}^{r-1} \sum_{k=s+2}^{n}\left[a_{s+1, k}(w) a_{s k}^{*}(w), a_{j r}(z) a_{j, r+1}^{*}(z)\right]=0$,

(l) $\sum_{j=r+2}^{n} \sum_{k=1}^{s-1}\left[a_{k s}(w) a_{k, s+1}^{*}(w), a_{r+1, j}(z) a_{r+1, j}^{*}(z)\right]=0$, 
(m) $\sum_{j=r+2}^{n} \sum_{k=s+2}^{n}\left[a_{s+1, k}(w) a_{s k}^{*}(w), a_{r j}(z) a_{r j}^{*}(z)-a_{r+1, j}(z) a_{r+1, j}^{*}(z)\right]$

$$
\begin{aligned}
= & -2 \delta_{s r} \delta(w / z) \sum_{j=r+2}^{n} a_{r+1, j}(w) a_{r j}^{*}(z)+\delta_{s, r-1} \delta(w / z) \sum_{j=r+2}^{n} a_{r j}(z) a_{r-1, j}^{*}(w) \\
& +\delta_{s, r+1} \delta(w / z) \sum_{j=r+3}^{n} a_{r+2, j}(w) a_{r+1, j}^{*}(z),
\end{aligned}
$$

(n) $\sum_{j=1}^{r-1}\left[a_{s, s+1}^{*}(w), a_{j r}(z) a_{j, r+1}^{*}(z)\right]=-\delta_{s, r-1} a_{r-1, r+1}^{*}(z) \delta(w / z)$,

(o) $\sum_{j=r+2}^{n}\left[a_{s, s+1}^{*}(w), a_{r+1, j}(z) a_{r j}^{*}(z)\right]=-\delta_{r+1, s} a_{r, r+2}^{*}(z) \delta(w / z)$.

Agora estamos em condições de verificar $\left(R_{1}\right)-\left(R_{6}\right)$.

Lema 2.2.11. $\left(R_{1}\right)\left[\rho_{\mathbb{J}}\left(H_{r}\right)(z), \rho_{\mathbb{J}}\left(H_{s}\right)(w)\right]=-\left(H_{r} \mid H_{s}\right) c \dot{\delta}(z / w)$.

Demonstração. Observe que se $|r-s|>1$, então $\left[\rho_{\mathbb{J}}\left(H_{r}\right)(z), \rho_{\mathbb{J}}\left(H_{s}\right)(w)\right]=0$ já que todos os somandos são iguais a zero. Escreva em termos de $\delta_{s, f(r)}$. Temos o seguinte

$$
\begin{aligned}
{\left[\rho_{\mathbb{J}}\left(H_{r}\right)(z), \rho_{\mathbb{J}}(\right.} & \left.\left.H_{s}\right)(w)\right]=\delta_{s r}\left(\left[\rho_{\mathbb{J}}\left(H_{r}\right)(z), \rho_{\mathbb{J}}\left(H_{r}\right)(w)\right]\right) \\
+ & \delta_{s, r-1}\left(\left[\rho_{\mathbb{J}}\left(H_{r}\right)(z), \rho_{\mathbb{J}}\left(H_{r-1}\right)(w)\right]\right)+\delta_{s, r+1}\left(\left[\rho_{\mathbb{J}}\left(H_{r}\right)(z), \rho_{\mathbb{J}}\left(H_{r+1}\right)(w)\right]\right) \\
= & \delta_{s r}\left(\left[2 a_{r, r+1}(z) a_{r, r+1}^{*}(z), \rho_{\mathbb{J}}\left(H_{r}\right)(w)\right]\right. \\
& +\sum_{i=1}^{r-1}\left[a_{i, r+1}(z) a_{r, r+1}^{*}(z)-a_{i, r}(z) a_{i, r}^{*}(z), \rho_{\mathbb{J}}\left(H_{r}\right)(w)\right] \\
& +\sum_{j=r+2}^{n}\left[a_{r j}(z) a_{r j}^{*}(z)-a_{r+1, j}(z) a_{r+1, j}^{*}(z), \rho_{\mathbb{J}}\left(H_{r}\right)(w)\right] \\
+ & {\left.\left[-\gamma b_{r}(z)+\frac{\gamma}{2}\left(b_{r-1}^{+}(z)+b_{r+1}^{+}(z)\right), \rho_{\mathbb{J}}\left(H_{r}\right)(w)\right]\right) } \\
+ & \delta_{s, r-1}\left(\left[2 a_{r, r+1}(z) a_{r, r+1}^{*}(z), \rho_{\mathbb{J}}\left(H_{r-1}\right)(w)\right]\right. \\
+ & \sum_{i=1}^{r-1}\left[a_{i, r+1}(z) a_{i, r+1}^{*}(z)-a_{i, r}(z) a_{i, r}^{*}(z), \rho_{\mathbb{J}}\left(H_{r-1}\right)(w)\right] \\
+ & \sum_{j=r+2}^{n}\left[a_{r j}(z) a_{r j}^{*}(z)-a_{r+1, j}(z) a_{r+1, j}^{*}(z), \rho_{\mathbb{J}}\left(H_{r-1}\right)(w)\right] \\
+ & {\left.\left[-\gamma b_{r}(z)+\frac{\gamma}{2}\left(b_{r-1}^{+}(z)+b_{r+1}^{+}(z)\right), \rho_{\mathbb{J}}\left(H_{r-1}\right)(w)\right]\right) } \\
+ & \delta_{s, r+1}\left(\left[\rho_{\mathbb{J}}\left(H_{r}\right)(z), \rho_{\mathbb{J}}\left(H_{r+1}\right)(w)\right]\right) .
\end{aligned}
$$

Reagrupando os termos, temos 


$$
\begin{aligned}
{\left[\rho_{\mathbb{J}}\left(H_{r}\right)\right.} & \left.(z), \rho_{\mathbb{J}}\left(H_{s}\right)(w)\right]=\delta_{s r}\left(4\left[a_{r, r+1}(z) a_{r, r+1}^{*}(z), a_{r, r+1}(w) a_{r, r+1}^{*}(w)\right]\right. \\
+ & \sum_{i=1}^{r-1}\left[a_{i, r+1}(z) a_{r, r+1}^{*}(z), a_{i, r+1}(w) a_{i, r+1}^{*}(w)\right] \\
+ & \sum_{i=1}^{r-1}\left[a_{i, r}(z) a_{i, r}^{*}(z), a_{i, r}(w) a_{i, r}^{*}(w)\right]+0+\gamma^{2}\left[b_{r}(z), b_{r}(w)\right] \\
+ & \left.\frac{\gamma^{2}}{4}\left[b_{r-1}^{+}(z), b_{r-1}^{+}(w)\right]+\frac{\gamma^{2}}{4}\left[b_{r+1}^{+}(z), b_{r+1}^{+}(w)\right]\right) \\
+ & \delta_{s, r-1}\left(\left[2 a_{r, r+1}(z) a_{r, r+1}^{*}(z), \sum_{j=r+1}^{n}-a_{(r-1)+1, j}(w) a_{(r-1)+1, j}^{*}(w)\right]\right. \\
+ & \sum_{i=1}^{r-1}\left[-a_{i, r}(z) a_{i, r}^{*}(z), \sum_{j=1}^{r-2}-a_{j, r}(w) a_{j, r}^{*}(w)\right] \\
+ & \sum_{j=r+2}^{n}\left[a_{r j}(z) a_{r j}^{*}(z), \sum_{i=r+1}^{n}-a_{r i}(w) a_{r i}^{*}(w)\right] \\
+ & {\left.\left[-\gamma b_{r}(z), \frac{\gamma}{2} b_{(r-1)+1}^{+}(w)\right]+\left[\frac{\gamma}{2} b_{r-1}^{+}(z),-\gamma b_{r-1}(w)\right]\right) } \\
+ & \delta_{s, r+1}\left(\left[\rho_{\mathbb{J}}\left(H_{r}\right)(z), \rho_{\mathbb{J}}\left(H_{r+1}\right)(w)\right]\right) .
\end{aligned}
$$

Finalmente,

$$
\begin{aligned}
{\left[\rho_{\mathbb{J}}\left(H_{r}\right)(z), \rho_{\mathbb{J}}(\right.} & \left.\left.H_{s}\right)(w)\right]=\delta_{s r}(0+0+0+0+2 c \dot{\delta}(w / z)+0+0) \\
& +\delta_{s, r-1}\left(-2\left[a_{r, r+1}(z) a_{r, r+1}^{*}(z), a_{r, r+1}(w) a_{r, r+1}^{*}(w)\right]\right. \\
& -\sum_{i=1}^{r-2}\left[a_{i, r}(z) a_{i, r}^{*}(z), a_{i, r}(w) a_{i, r}^{*}(w)\right] \\
& +\sum_{j=r+2}^{n}\left[a_{r j}(z) a_{r j}^{*}(z),-a_{r j}(w) a_{r j}^{*}(w)\right] \\
& \left.-c \dot{\delta}^{-}(w / z)+c \dot{\delta}^{-}(z / w)\right)+\delta_{s, r+1}\left(\left[\rho_{\mathbb{J}}\left(H_{r}\right)(z), \rho_{\mathbb{J}}\left(H_{r+1}\right)(w)\right]\right) \\
= & \delta_{s r}(2 c \dot{\delta}(w / z))+\delta_{s, r-1}\left(0+0+0+-c \dot{\delta}^{-}(w / z)-c \dot{\delta}^{+}(w / z)\right) \\
& +\delta_{s, r+1}\left(\left[\rho_{\mathbb{J}}\left(H_{r}\right)(z), \rho_{\mathbb{J}}\left(H_{r+1}\right)(w)\right]\right) \\
= & \delta_{s r}(2 c \dot{\delta}(w / z))+\delta_{s, r-1}(-c \dot{\delta}(w / z))+\delta_{s, r+1}(-c \dot{\delta}(w / z)),
\end{aligned}
$$

o que nos dá

$$
\left[\rho_{\mathbb{J}}\left(H_{r}\right)(z), \rho_{\mathbb{J}}\left(H_{r+1}\right)(w)\right]=-\left[\rho_{\mathbb{J}}\left(H_{r+1}\right)(w), \rho_{\mathbb{J}}\left(H_{r}\right)(z)\right]=c \dot{\delta}(z / w)=-c \dot{\delta}(w / z) .
$$

Desta forma, a relação $\left(R_{1}\right)$ está verificada.

Lema 2.2.12. $\left(R_{2}\right)\left[\rho_{\mathbb{J}}\left(H_{r}\right)(z), \rho_{\mathbb{J}}\left(E_{s}\right)(w)\right]=C_{r s} \rho_{\mathbb{J}}\left(E_{s}\right)(z) \delta(z / w)$. 
Demonstração. Temos o seguinte

(a) $2\left[a_{r, r+1}(z) a_{r, r+1}^{*}(z), \rho_{\mathbb{J}}\left(E_{s}\right)(w)\right]$

$$
\begin{aligned}
= & \delta_{s r}\left(2\left[a_{r, r+1}(z) a_{r, r+1}^{*}(z), a_{r, r+1}(w) a_{r, r+1}^{*}(w) a_{r, r+1}^{*}(w)\right]\right. \\
& +2\left[a_{r, r+1}(z) a_{r, r+1}^{*}(z),\left(\sum_{j=r+2}^{n} a_{r j}(w) a_{r j}^{*}(w)-a_{r+1, j}(w) a_{r+1, j}^{*}(w)\right) a_{r, r+1}^{*}(w)\right] \\
& \left.+2\left[a_{r, r+1}(z) a_{r, r+1}^{*}(z),-\gamma a_{r, r+1}^{*}(w)\left(\frac{1}{2}+b_{r}(w)-\frac{1}{2} b_{r-1}^{+}(w)-\frac{1}{2} b_{r+1}^{+}(w)\right)\right]\right) \\
& +\delta_{s, r+1}\left(2\left[a_{r, r+1}(z) a_{r, r+1}^{*}(z), a_{r, r+1}(w) a_{r, r+2}^{*}(w)\right]\right) \\
& +\delta_{s, r-1}\left(-2 a_{r-1, r+1}^{*}(z) a_{r, r+1}(w) \delta(w / z)\right)
\end{aligned}
$$

(h)

$\stackrel{(g)}{=} \delta_{s, r}\left(2 a_{r, r+1}(w) a_{r, r+1}^{*}(z) a_{r, r+1}^{*}(w) \delta(w / z)\right.$

$+2 \sum_{j=r+2}^{n}\left(a_{r j}(w) a_{r j}^{*}(w)-a_{r+1, j}(w) a_{r+1, j}^{*}(w)\right) a_{r, r+1}^{*}(z) \delta(w / z)$

$-2 \gamma a_{r, r+1}^{*} b_{r}(w) \delta(w / z)+\gamma a_{r, r+1}^{*}(z)\left(b_{r-1}^{+}(w)+b_{r+1}^{+}(w)\right) \delta(w / z)$

$\left.-\gamma^{2} a_{r, r+1}^{*}(z) \dot{\delta}(w / z)\right)+\delta_{s, r+1}\left(2 a_{r, r+1}(z) a_{r, r+2}^{*}(w) \delta(w / z)\right)$

$+\delta_{s, r-1}\left(-2 a_{r-1, r+1}^{*}(z) a_{r, r+1}(w) \delta(w / z)\right)$.

(b) $\sum_{i=1}^{r-1}\left[a_{i, r+1}(z) a_{i, r+1}^{*}(z)-a_{i, r}(z) a_{i, r}^{*}(z), \rho_{\mathbb{J}}\left(E_{s}\right)(w)\right]$

$$
\begin{aligned}
= & \delta_{s r}\left(\sum_{i=1}^{r-1}\left[a_{i, r+1}(z) a_{i, r+1}^{*}(z)-a_{i, r}(z) a_{i, r}^{*}(z), \sum_{j=1}^{r-1} a_{j r}(w) a_{j, r+1}^{*}(w)\right]\right) \\
& +\delta_{s, r+1}\left(\sum_{i=1}^{r-1}\left[a_{i, r+1}(z) a_{i, r+1}^{*}(z), a_{i, r+1}(w) a_{i, r+2}^{*}(w)\right]\right) \\
& +\delta_{s, r-1}\left(-a_{r-1, r}(w) a_{r-1, r}^{*}(z) a_{r-1, r}^{*}(w)\right. \\
& +\sum_{j=r+1}^{n}\left(a_{r-1, j}(w) a_{r-1, j}^{*}(w)-a_{r j}(w) a_{r j}^{*}(w)\right) a_{r-1, r}^{*}(z) \\
& +\sum_{j=1}^{r-2} a_{j, r-1}(w) a_{j r}^{*}(z)-a_{r, r+1}(w) a_{r-1, r+1}^{*}(z)-\gamma a_{r-1}^{*}(w) b_{r-1}(w) \\
& \left.+\frac{\gamma}{2}\left(a_{r-1, r}^{*}(w) b_{r-2}^{+}(w)-a_{r-1, r}^{*}(w) b_{r}^{+}(w)\right) \delta(w / z)+\frac{\gamma^{2}}{2} a_{r-1, r}^{*}(z) \dot{\delta}(w / z)\right)
\end{aligned}
$$




$$
\begin{aligned}
& =\delta_{s r}\left(2 \sum_{i=1}^{r-1} a_{i r}(w) a_{i, r+1}^{*}(w) \delta(w / z)\right)+\delta_{s, r+1}\left(-\sum_{i=1}^{r-1} a_{i, r+1}(z) a_{i, r+2}^{*}(w) \delta(w / z)\right) \\
& +\delta_{s, r-1}\left(-a_{r-1, r}(w) a_{r-1, r}^{*}(z) a_{r-1, r}^{*}(w)\right. \\
& +\sum_{j=r+1}^{n}\left(a_{r-1, j}(w) a_{r-1, j}^{*}(w)-a_{r j}(w) a_{r j}^{*}(w)\right) a_{r-1, r}^{*}(z) \\
& +\sum_{j=1}^{r-2} a_{j, r-1}(w) a_{j r}^{*}(z)-a_{r, r+1}(w) a_{r-1, r+1}^{*}(z)-\gamma a_{r-1}^{*}(w) b_{r-1}(w) \\
& \left.+\frac{\gamma}{2}\left(a_{r-1, r}^{*}(w) b_{r-2}^{+}(w)-a_{r-1, r}^{*}(w) b_{r}^{+}(w)\right) \delta(w / z)+\frac{\gamma^{2}}{2} a_{r-1, r}^{*}(z) \dot{\delta}(w / z)\right) . \\
& \text { (c) } \sum_{j=r+2}^{n}\left[a_{r j}(z) a_{r j}^{*}(z)-a_{r+1, j}(z) a_{r+1, j}^{*}(z), \rho_{\mathrm{J}}\left(E_{s}\right)(w)\right] \\
& =\delta_{s r}\left(\sum_{j=r+2}^{n}\left[-a_{r+1, j}(z) a_{r+1, j}^{*}(z),-a_{r+1, j}(w) a_{r+1, j}^{*}(w)\right]\right. \\
& +\sum_{j=r+2}^{n}\left[a_{r j}(z) a_{r j}^{*}(z), a_{r j}(w) a_{r j}^{*}(w)\right]+\sum_{j=r+2}^{n}\left[a_{r j}(z) a_{r j}^{*}(z)-a_{r+1, j}(z) a_{r+1, j}^{*}(z),\right. \\
& \left.\left.\left(a_{r j}(w) a_{r j}^{*}(w)-a_{r+1, j}(w) a_{r+1, j}^{*}(w)\right) a_{r, r+1}^{*}(w)\right]\right) \\
& +\delta_{s, r+1}\left(-a_{r+1, r+2}(w) a_{r+1, r+2}^{*}(z) a_{r+1, r+2}^{*}(w)\right. \\
& +\sum_{j=r+3}^{n}\left(a_{r+1, j}(w) a_{r+1, j}^{*}(w)-a_{r+2, j}(w) a_{r+2, j}^{*}(w)\right) a_{r+1, r+2}^{*}(z) \\
& -a_{r, r+1}(w) a_{r, r+2}^{*}(z)-\sum_{j=r+3}^{n} a_{r+2, j}(w) a_{r+1, j}^{*}(w) \\
& -\gamma a_{r+1, r+2}^{*}(w) b_{r+1}(w)+\frac{\gamma}{2} a_{r+1, r+2}^{*}(w)\left(b_{r}^{+}(w)+b_{r+2}^{+}(w)\right) \delta(w / z) \\
& \left.+\frac{\gamma^{2}}{2} a_{r+1, r+2}^{*}(z) \dot{\delta}(w / z)\right)+\delta_{s, r-1}\left(\sum_{j=r+2}^{n} a_{r j}(w) a_{r-1, j}^{*}(w) \delta(w / z)\right)
\end{aligned}
$$




$$
\begin{aligned}
=\delta_{s r}( & \left.0+0+2 \sum_{j=r+2}^{n} a_{r+1, j}(w) a_{r j}^{*}(z) \delta(w / z)\right) \\
& +\delta_{s, r+1}\left(-a_{r+1, r+2}(w) a_{r+1, r+2}^{*}(z) a_{r+1, r+2}^{*}(w)\right. \\
& +\sum_{j=r+3}^{n}\left(a_{r+1, j}(w) a_{r+1, j}^{*}(w)-a_{r+2, j}(w) a_{r+2, j}^{*}(w)\right) a_{r+1, r+2}^{*}(z) \\
& -a_{r, r+1}(w) a_{r, r+2}^{*}(z)-\sum_{j=r+3}^{n} a_{r+2, j}(w) a_{r+1, j}^{*}(w) \\
& -\gamma a_{r+1, r+2}^{*}(w) b_{r+1}(w)+\frac{\gamma}{2} a_{r+1, r+2}^{*}(w)\left(b_{r}^{+}(w)+b_{r+2}^{+}(w)\right) \delta(w / z) \\
& \left.+\frac{\gamma^{2}}{2} a_{r+1, r+2}^{*}(z) \dot{\delta}(w / z)\right)+\delta_{s, r-1}\left(\sum_{j=r+2}^{n} a_{r j}(w) a_{r-1, j}^{*}(w) \delta(w / z)\right) .
\end{aligned}
$$

Finalmente,

$$
\begin{aligned}
(d)\left[-\gamma b_{r}(z)+\right. & \left.\frac{\gamma}{2}\left(b_{r-1}^{+}(z)+b_{r+1}^{+}(z)\right), \rho_{\mathbb{J}}\left(E_{s}\right)(w)\right]=\delta_{s r}\left(\gamma^{2}\left[b_{r}(z), a_{r, r+1}^{*}(w) b_{r}(w)\right]\right) \\
& +\delta_{s, r+1}\left(-\frac{\gamma^{2}}{2} a_{r+1, r+2}^{*}(w)\left(\left[b_{r}(z), b_{r}^{+}(w)\right]+\left[b_{r+1}^{+}(z), b_{r+1}(w)\right]\right)\right) \\
& +\delta_{s, r-1}\left(\frac{\gamma^{2}}{2} a_{r-1, r}^{*}(w)\left(\left[b_{r}(z), b_{r}^{+}(w)\right]+\left[b_{r-1}^{+}(z), b_{r-1}(w)\right]\right)\right) \\
= & \delta_{s r}\left(\gamma^{2} a_{r, r+1}^{*}(w) \dot{\delta}(w / z)\right)+\delta_{s, r+1}\left(-\frac{\gamma^{2}}{2} a_{r+1, r+2}^{*}(w) \dot{\delta}(w / z)\right) \\
& +\delta_{s, r-1}\left(\frac{\gamma^{2}}{2} a_{r-1, r}^{*}(w) \dot{\delta}(w / z)\right) .
\end{aligned}
$$

Somando as quatro equações, temos

$$
\begin{aligned}
{\left[\rho_{\mathrm{J}}\left(H_{r}\right)(z), \rho_{\mathrm{J}}\left(E_{s}\right)(w)\right]=} & \delta_{s r} 2 \rho_{\mathrm{J}}\left(E_{r}\right)(z) \delta(w / z)+\delta_{s, r+1}\left(-\rho_{\mathrm{J}}\left(E_{r+1}\right)(z) \delta(w / z)\right) \\
& +\delta_{s, r-1}\left(-\rho_{\mathbb{J}}\left(E_{r-1}\right)(z) \delta(w / z)\right),
\end{aligned}
$$

o que prova $\left(R_{2}\right)$.

Lema 2.2.13. $\left(R_{3}\right)\left[\rho_{\mathbb{J}}\left(H_{r}\right)(z), \rho_{\mathbb{J}}\left(F_{s}\right)(w)\right]=-C_{r s} \rho_{\mathbb{J}}\left(F_{s}\right)(z) \delta(z / w)$.

Demonstração. Temos

(a) $2\left[a_{r, r+1}(z) a_{r, r+1}^{*}(z), \rho_{\mathbb{J}}\left(F_{s}\right)(w)\right]=\delta_{s r}\left(2 a_{r, r+2}(z) \delta(z / w)\right)+\delta_{s, r-1} 0$

$$
\begin{aligned}
& +\delta_{s, r+1}\left(2\left[a_{r, r+1}(z) a_{r, r+1}^{*}(z), a_{r, r+2}(w) a_{r, r+1}^{*}(w)\right]\right) \\
= & \delta_{s r}\left(2 a_{r, r+2}(z) \delta(z / w)\right)+\delta_{s, r+1}\left(2 a_{r, r+2}(w) a_{r, r+1}^{*}(z) \delta(w / z)\right) .
\end{aligned}
$$




$$
\begin{aligned}
& \text { (b) } \sum_{i=1}^{r-1}\left[a_{i, r+1}(z) a_{i, r+1}^{*}(z)-a_{i, r}(z) a_{i, r}^{*}(z), \rho_{\mathbb{J}}\left(F_{s}\right)(w)\right] \\
& =\delta_{s r}\left(\sum_{i=1}^{r-1}\left[a_{i, r+1}(z) a_{i, r+1}^{*}(z), a_{i, r+1}(w) a_{i, r}^{*}(w)\right]-\sum_{i=1}^{r-1}\left[a_{i, r}(z) a_{i, r}^{*}(z), a_{i, r+1}(w) a_{i, r}^{*}(w)\right]\right) \\
& +\delta_{s, r+1}\left(\sum_{j=1}^{r}\left[a_{i, r+1}(z) a_{i, r+1}^{*}(z), a_{j, r+2}(w) a_{j, r+1}^{*}(w)\right]\right) \\
& +\delta_{s, r-1}\left(\left[a_{r-1, r}(z) a_{r-1, r}^{*}(z), a_{r-1, r}(w)\right]-\sum_{i=1}^{r-2} a_{i r}(z)\left[a_{i r}^{*}(z), a_{i r}(w)\right] a_{i, r-1}^{*}(w)\right) \\
& =\delta_{s r}\left(-2 \sum_{i=1}^{r-1} a_{i, r+1}(z) a_{i r}^{*}(w) \delta(z / w)\right)+\delta_{s, r+1}\left(\sum_{i=1}^{r-1} a_{i, r+2}(w) a_{i, r+1}^{*}(z) \delta(z / w)\right) \\
& +\delta_{s, r-1}\left(\left(-a_{r-1, r}(z)+\sum_{i=1}^{r-2} a_{i r}(z) a_{i, r-1}^{*}(w)\right) \delta(z / w)\right) . \\
& \text { (c) } \sum_{j=r+2}^{n}\left[a_{r j}(z) a_{r j}^{*}(z)-a_{r+1, j}(z) a_{r+1, j}^{*}(z), \rho_{\mathrm{J}}\left(E_{s}\right)(w)\right] \\
& =\delta_{s r} 0+\delta_{s, r+1}\left(\sum_{j=r+2}^{n} \sum_{k=1}^{r}\left[a_{r, j}(z) a_{r, j}^{*}(z), a_{k, r+2}(w) a_{k, r+1}^{*}(w)\right]\right. \\
& \left.+\sum_{j=r+2}^{n}\left[a_{r+1, j}(z) a_{r+1, j}^{*}(z), a_{r+1, r+2}(w)\right]\right)+\delta_{s, r-1} 0 \\
& =\delta_{s, r+1}\left(-a_{r, r+2}(z) a_{r, r+1}^{*}(w) \delta(z / w)-a_{r+1, r+2}(z) \delta(z / w)\right) . \\
& (d)\left[-\gamma b_{r}(z)+\frac{\gamma}{2}\left(b_{r-1}^{+}(z)+b_{r+1}^{+}(z)\right), \rho_{\mathbb{J}}\left(F_{s}\right)(w)\right]=0 .
\end{aligned}
$$

Somando as quatro parcelas, temos o resultado.

Lema 2.2.14. $\left(R_{4}\right)\left[\rho_{\mathbb{J}}\left(E_{r}\right)(z), \rho_{\mathbb{J}}\left(F_{s}\right)(w)\right]=\delta_{s r}\left(\rho_{\mathbb{J}}\left(H_{r}\right)(z) \delta(z / w)-c \dot{\delta}(z / w)\right)$.

Demonstração. Se $|s-r|>1$, então $\left[\rho\left(E_{r}\right)(z),-a_{r, r+1}(w)\right]=0$. Desta forma,

$$
\sum_{j=1}^{r-1} \sum_{k=1}^{s-1}\left[a_{k r}(z) a_{k, r+1}^{*}(z), a_{j, s+1}(w) a_{j s}^{*}(w)\right]=0
$$

já que $r \neq s$. Assim 


$$
\begin{aligned}
\sum_{j=1}^{s-1}[ & \sum_{k=r+2}^{n}\left(a_{r k}(z) a_{r k}^{*}(z)-a_{r+1, k}(z) a_{r+1, k}^{*}(z)\right) a_{r, r+1}^{*}(z) \\
& \left.+\sum_{k=r+2}^{n} a_{r+1, k}(z) a_{r k}^{*}, a_{j, s+1}(w) a_{j s}^{*}(w)\right] \\
= & \sum_{j=1}^{s-1} \sum_{k=r+2}^{n}\left[a_{r k}(z) a_{r k}^{*}(z)-a_{r+1, k}(z) a_{r+1, k}^{*}(z), a_{j, s+1}(w) a_{j s}^{*}(w)\right] a_{r, r+1}^{*}(z) \\
& -\sum_{j=1}^{s-1} \sum_{k=r+2}^{n}\left[a_{r+1, k}(z) a_{r k}^{*}(z), a_{j, s+1}(w) a_{j s}^{*}(w)\right] \\
= & \sum_{j=1}^{s-1} \sum_{k=r+2}^{n}\left(a_{j, s+1}(w) a_{r k}^{*}(z) \delta_{s k} \delta_{r j}-a_{r k}(z) a_{j s}^{*}(w) \delta_{s+1, k} \delta_{j r}\right. \\
& \left.-a_{j, s+1}(w) a_{r+1, k}^{*}(z) \delta_{r+1, j} \delta_{k s}+a_{r+1, k}(z) a_{j s}^{*}(w) \delta_{r+1, j} \delta_{k, s+1}\right) a_{r, r+1}^{*}(z) \delta(z / w) \\
& +\sum_{j=1}^{n-1} \sum_{k=r+2}^{n}\left(a_{r+1, k}(z) a_{j s}^{*}(w) \delta_{r j} \delta_{k, s+1}-a_{j, s+1}(w) a_{r k}^{*}(z) \delta_{r+1, j} \delta_{k s}\right) \delta(z / w) \\
= & \circledast .
\end{aligned}
$$

Se $s+1>n$, então $a_{j, s+1}(w)=0=\delta_{k, s+1}$ e $\circledast=0$. Agora suponhamos que $s+1 \leqslant n$. Se $s-1<r$ então $j \leqslant s-1<r, \delta_{j r}=\delta_{j, r+1}=0$ e $\circledast=0$. Se $s-1 \geqslant r$, temos $s-1>r$ já que $|s-r|>1$. Então $s \geqslant r+2$ e por esta razão os termos $j=r, r+1$ e $k=s, s+1$ aparecem na expressão. Então

$$
\begin{aligned}
\circledast= & \left(a_{r, s+1}(w) a_{r s}^{*}(z)-a_{r, s+1}(z) a_{r s}^{*}(w)-a_{r+1, s+1}(w) a_{r+1, s}^{*}(z)\right. \\
& \left.+a_{r+1, s+1}(z) a_{r+1, s}^{*}(w)\right) a_{r, r+1}^{*}(z) \delta(z / w) \\
& +\left(a_{r+1, s+1}(z) a_{r s}^{*}(w)-a_{r+1, s+1}(w) a_{r s}^{*}(z)\right) \delta(z / w) \\
= & 0
\end{aligned}
$$

logo temos expressões não-triviais apenas quando $r=s, r=s-1$ ou $r=s+1$. Desta forma,

$$
\begin{aligned}
& {\left[\rho_{\mathbb{J}}\left(E_{r}\right)(z), \rho_{\mathbb{J}}\left(F_{s}\right)(w)\right]=\delta_{s r}\left(\left[\rho_{\mathbb{J}}\left(E_{r}(z)\right),-a_{r, r+1}(w)\right]\right.} \\
& \left.+\left[\rho_{\mathbb{J}}\left(E_{r}(z)\right), \sum_{j=1}^{r-1} a_{j, r+1}(w) a_{j r}^{*}(w)\right]\right)+\delta_{s, r+1}\left(\left[\rho_{\mathbb{J}}\left(E_{s-1}(z)\right),-a_{s, s+1}(w)\right]\right. \\
& \left.+\left[\rho_{\mathbb{J}}\left(E_{s-1}(z)\right), \sum_{j=1}^{s-1} a_{j, s+1}(w) a_{j s}^{*}(w)\right]\right)+\delta_{s, r-1}\left(\left[\rho_{\mathbb{J}}\left(E_{s+1}\right)(z),-a_{s, s+1}(w)\right]\right. \\
& \left.+\left[\rho_{\mathbb{J}}\left(E_{s+1}\right)(z), \sum_{j=1}^{s-1} a_{j, s+1}(w) a_{j s}^{*}(w)\right]\right)
\end{aligned}
$$




$$
\begin{aligned}
= & \delta_{s r}\left(\left(2 a_{r, r+1}(z) a_{r, r+1}^{*}(z)+\sum_{j=r+2}^{n}\left(a_{r j}^{*}(z) a_{r j}(z)-a_{r+1, j}^{*}(z) a_{r+1, j}(z)\right)\right.\right. \\
& \left.-\gamma b_{r}(z)+\frac{\gamma}{2}\left(b_{r-1}^{+}(z)+b_{r+1}^{+}(z)\right)\right) \delta(z / w)-\frac{\gamma^{2}}{2} \dot{\delta}(z / w) \\
& \left.+\sum_{j=1}^{r-1}\left[a_{j r}(z) a_{j, r+1}^{*}(z), a_{j, r+1}(w) a_{j r}^{*}(w)\right]\right) \\
& +\delta_{s, r+1}\left(-\sum_{j=s+1}^{n}\left[a_{s, s+1}(w), a_{s j}(z) a_{s-1, s}^{*}(z) a_{s j}^{*}(z)\right]+a_{s, s+1}(z) a_{s-1, s}^{*}(z) \delta(w / z)\right) \\
= & \delta_{s r}\left(\left(2 a_{r, r+1}(z) a_{r, r+1}^{*}(z)+\sum_{j=r+2}^{n}\left(a_{r j}^{*}(z) a_{r j}(z)-a_{r+1, j}^{*}(z) a_{r+1, j}(z)\right)\right.\right. \\
& \left.-\gamma b_{r}(z)+\frac{\gamma}{2}\left(b_{r-1}^{+}(z)+b_{r+1}^{+}(z)\right)\right) \delta(z / w)-\frac{\gamma^{2}}{2} \dot{\delta}(z / w) \\
& \left.+\sum_{j=1}^{r-1}\left(a_{j, r+1}(z) a_{j, r+1}^{*}(z)-a_{j r}(z) a_{j r}^{*}(z)\right) \delta(z / w)\right) \\
& +\delta_{s, r+1}\left(-a_{s, s+1}(z) a_{s-1, s}^{*}(z) \delta(w / z)+a_{s, s+1}(z) a_{s-1, s}^{*}(z) \delta(w / z)\right) \\
= & \delta_{s r}\left(\rho_{\mathbb{J}}\left(H_{r}\right)(z) \delta(z / w)-c \dot{\delta}(z / w)\right),
\end{aligned}
$$

o que prova o Lema.

Lema 2.2.15. Valem as relações

$$
\begin{aligned}
\left(R_{5} \mid R_{6}\right) & {\left[\rho_{\mathbb{J}}\left(F_{r}\right)(z), \rho_{\mathbb{J}}\left(F_{s}\right)(w)\right]=\left[\rho_{\mathbb{J}}\left(E_{r}\right)(z), \rho_{\mathbb{J}}\left(E_{s}\right)(w)\right]=0 \text { se } C_{r s} \neq-1, } \\
& {\left[\rho_{\mathbb{J}}\left(F_{r}\right)\left(z_{1}\right), \rho_{\mathbb{J}}\left(F_{r}\right)\left(z_{2}\right), \rho_{\mathbb{J}}\left(F_{s}\right)(w)\right]=\left[\rho_{\mathbb{J}}\left(E_{r}\right)\left(z_{1}\right), \rho_{\mathbb{J}}\left(E_{r}\right)\left(z_{2}\right), \rho_{\mathbb{J}}\left(E_{s}\right)(w)\right]=0 } \\
& s e C_{r s}=-1 .
\end{aligned}
$$

Demonstração. Temos

$$
\begin{aligned}
& {\left[\rho_{\mathbb{J}}\left(F_{r}\right)(z), \rho_{\mathbb{J}}\left(F_{s}\right)(w)\right]=\left[-a_{r, r+1}(z), \rho_{\mathbb{J}}\left(F_{s}\right)(w)\right]+\left[\sum_{j=1}^{r-1} a_{j, r+1}(z) a_{j r}^{*}(z), \rho_{\mathbb{J}}\left(F_{s}\right)(w)\right] } \\
&=\delta_{s, r+1} a_{r, r+2}(w) \delta(w / z)+\delta_{s, r-1} a_{r-1, r+1}(w) \delta(w / z) \\
&-\delta_{s, r-1} \sum_{j=1}^{s-1} a_{j, r+1}(w) a_{j s}^{*}(w) \delta(w / z)+\delta_{s, r+1} \sum_{j=1}^{r-1} a_{j, r+2}(w) a_{j r}^{*}(w) \delta(w / z) \\
&=\left(\delta_{s, r+1}\left(a_{r, r+2}(w)+\sum_{j=1}^{r-1} a_{j, r+2}(w) a_{j r}^{*}(w)\right)\right. \\
&\left.+\delta_{s, r-1}\left(a_{r-1, r+1}(w)-\sum_{j=1}^{r-2} a_{j, r+1}(w) a_{j, r-1}^{*}(w)\right)\right) \delta(w / z) .
\end{aligned}
$$

Então $\left[\rho_{\mathbb{J}}\left(F_{r}\right)(z), \rho_{\mathbb{J}}\left(F_{s}\right)(w)\right]=0$ se $|r-s| \neq 1$, isto é, se $C_{r s} \neq-1$. Agora 


$$
\begin{aligned}
& {\left[\rho_{\mathbb{J}}\left(E_{r}\right)(z), \rho_{\mathbb{J}}\left(E_{s}\right)(w)\right]=\left[a_{r, r+1}(z) a_{r, r+1}^{*}(z)^{2}, \rho_{\mathbb{J}}\left(E_{s}\right)(w)\right]} \\
& +\left[\sum_{j=r+2}^{n}\left(a_{r j}(z) a_{r j}^{*}(z)-a_{r+1, j}(z) a_{r+1, j}^{*}(z)\right) a_{r, r+1}^{*}(z), \rho_{\mathbb{J}}\left(E_{s}\right)(w)\right] \\
& +\left[\sum_{j=1}^{r-1} a_{j r}(z) a_{j, r+1}^{*}(z)-\sum_{j=r+2}^{n} a_{r+1, j}(z) a_{r j}^{*}(z), \rho_{\mathbb{J}}\left(E_{s}\right)(w)\right] \\
& +\left[-\gamma a_{r, r+1}^{*}(z)\left(b_{r}(z)-\frac{1}{2} a_{r, r+1}^{*}(z)\left(b_{r-1}^{+}(z)+b_{r+1}^{+}(z)\right)\right)-\frac{\gamma^{2}}{2} \dot{a}_{r, r+1}^{*}(z), \rho_{\mathbb{J}}\left(E_{s}\right)(w)\right] \\
& =-\delta_{s r} \sum_{j=r+2}^{n}\left(a_{r j}(w) a_{r j}^{*}(w)-a_{r+1, j}(w) a_{r+1, j}^{*}(w)\right) a_{r, r+1}^{*}(z)^{2} \delta(w / z) \\
& -\delta_{s, r-1} a_{r, r+1}(w) a_{r-1, r}^{*}(w) a_{r, r+1}^{*}(z)^{2} \delta(w / z)+2 \delta_{s, r+1} a_{r, r+1}(z) a_{r, r+1}^{*}(z) a_{r, r+2}^{*}(w) \delta(w / z) \\
& -2 \delta_{s, r-1} a_{r, r+1}(z) a_{r, r+1}^{*}(z) a_{r-1, r+1}^{*}(w) \delta(w / z) \\
& -\delta_{s r} \gamma a_{r, r+1}^{*}(z)^{2}\left(b_{r}(w)-\frac{1}{2}\left(b_{r-1}^{+}(w)+b_{r+1}^{+}(w)\right)\right) \delta(w / z)-\delta_{s r} \frac{\gamma^{2}}{2} a_{r, r+1}^{*}(z)^{2} \dot{\delta}(w / z) \\
& +\left(\delta_{s r} \sum_{j=r+2}^{n}\left(a_{r j}(w) a_{r j}^{*}(w)-a_{r+1, j}(w) a_{r+1, j}^{*}(w)\right) a_{r, r+1}^{*}(z)^{2}\right) \delta(w / z) \\
& +\delta_{s, r+1} a_{s, s+1}(w) a_{s, s+1}^{*}(z)^{2} a_{s, s+1}^{*}(w) \delta(w / z) \\
& +\delta_{s, r+1} \sum_{k=r+3}^{n}\left(a_{r+1, k}(w) a_{r+1, k}^{*}(w)-a_{r+2, k}(w) a_{r+2, j}^{*}(w)\right) a_{r, r+1}^{*}(z) a_{r+1, r+2}^{*}(z) \delta(w / z) \\
& -\delta_{s, r-1} \sum_{j=r+2}^{n}\left(a_{r j}(z) a_{r j}^{*}(z)-a_{r+1, j}(z) a_{r+1, j}^{*}(z)\right) a_{r-1, r}^{*}(w) a_{r, r+1}^{*}(w) \delta(w / z) \\
& +\sum_{j=r+2}^{n}\left(a_{r j}(z) a_{r j}^{*}(z)-a_{r+1, j}(z) a_{r+1, j}^{*}(z)\right) \\
& \cdot\left(\delta_{s, r-1} a_{r, r+2}^{*}(w)-\delta_{s, r-1} a_{r-1, r+1}^{*}(w)\right) \delta(w / z) \\
& -\delta_{s, r+1}\left(a_{r, r+1}(w) a_{r, r+2}^{*}(z)+\sum_{j=r+3}^{n} a_{r+2, k}(w) a_{r+1, j}^{*}(z)\right) a_{r, r+1}^{*}(z) \delta(w / z) \\
& +2 \delta_{s r} \sum_{j=r+2}^{n} a_{r+1, j}(w) a_{r j}^{*}(z) a_{r, r+1}^{*}(z) \delta(w / z)-\delta_{s, r-1} \sum_{j=r+2}^{n} a_{r j}(z) a_{r-1, j}^{*}(w) a_{r, r+1}^{*}(z) \delta(w / z) \\
& +a_{r, r+1}^{*}(z) a_{r+1, r+2}^{*}(z) \delta_{s, r+1}\left(\gamma b_{s}(w) \delta(w / z)-\frac{\gamma}{2}\left(b_{s-1}^{+}(w)+b_{s+1}^{+}(w)\right) \delta(w / z)\right. \\
& \left.+\frac{\gamma^{2}}{2} \dot{\delta}(w / z)\right)+\left(\left(-2 \delta_{s+1, r} a_{s, s+1}(z) a_{s, s+1}^{*}(z) a_{s, s+2}^{*}(w)\right.\right. \\
& +2 \delta_{r+1, s} a_{s, s+1}(z) a_{s, s+1}^{*}(z) a_{s-1, s+1}^{*}(w) \\
& -\delta_{s, r-1}\left(\sum_{j=s+2}^{n}\left(a_{s j}(z) a_{s j}^{*}(z)-a_{s+1, j}(z) a_{s+1, j}^{*}(z)\right) a_{s, s+2}^{*}(w)\right.
\end{aligned}
$$




$$
\begin{aligned}
& \left.+a_{s, s+1}(w) a_{s, s+1}^{*}(z) a_{s, s+2}^{*}(z)-\sum_{j=s+3}^{n} a_{s+2, k}(w) a_{s+1, j}^{*}(z) a_{s, s+1}^{*}(z)\right) \\
& +\delta_{s, r+1} \sum_{j=s+2}^{n} a_{s j}(z) a_{s-1, j}^{*}(w) a_{s, s+1}^{*}(z) \\
& +\delta_{s, r+1} \sum_{j=s+2}^{n}\left(a_{s j}(z) a_{s j}^{*}(z)-a_{s+1, j}(z) a_{s+1, j}^{*}(z)\right) a_{s-1, s+1}^{*}(w) \\
& +\delta_{s+1, r}\left(-\sum_{j=1}^{r-2} a_{j, r-1}(w) a_{j, r+1}^{*}(z)+\sum_{j=r+2}^{n} a_{r+1, j}(z) a_{r-1, j}^{*}(w)\right) \\
& \left.+\delta_{s, r+1}\left(\sum_{j=1}^{r-1} a_{j r}(z) a_{j, r+2}^{*}(w)-\sum_{j=r+3}^{n} a_{r+2, j}(w) a_{r j}^{*}(z)\right)\right) \delta(w / z) \\
& +\left(\delta_{r+1, s} a_{s-1, s+1}^{*}(z)-\delta_{s, r-1} a_{r-1, r+1}^{*}(z)\right)\left(\left(\gamma b_{s}(z)-\frac{\gamma}{2}\left(b_{s-1}^{+}(z)+b_{s+1}^{+}(z)\right)\right) \delta(w / z)\right. \\
& \left.\left.+\frac{\gamma}{2} \dot{\delta}(w / z)\right)-\frac{\gamma^{2}}{2} \delta_{s, r-1} \dot{a}_{r-1, r+1}(z) \delta(w / z)\right) \\
& +\left(\delta_{s r} \gamma a_{s, s+1}^{*}(z)^{2}\left(b_{s}(w)-\frac{1}{2}\left(b_{s-1}^{+}(w)+b_{s+1}^{+}(w)\right)\right) \delta(w / z)\right. \\
& +\delta_{s r} \frac{\gamma^{2}}{2} a_{s, s+1}^{*}(z)^{2} \dot{\delta}(w / z)-a_{s, s+1}^{*}(w) a_{s+1, s+2}^{*}(w) \delta_{s, r-1}\left(\gamma b_{r}(z) \delta(z / w)\right. \\
& \left.-\frac{\gamma}{2}\left(b_{r-1}^{+}(z)+b_{r+1}^{+}(z)\right) \delta(z / w)+\frac{\gamma^{2}}{2} \dot{\delta}(z / w)\right) \\
& +\left(\delta_{s, r+1} a_{s-1, s+1}^{*}(z)-\delta_{s+1, r} a_{r-1, r+1}^{*}(z)\right)\left(\left(\gamma b_{r}(z)-\frac{\gamma}{2}\left(b_{r-1}^{+}(z)+b_{r+1}^{+}(z)\right)\right) \delta(w / z)\right. \\
& \left.+\frac{\gamma^{2}}{2} \dot{\delta}(w / z)\right)-\frac{\gamma^{2}}{2} \delta_{s, r-1} \dot{a}_{r-1, r+1}(z) \delta(w / z) \\
& \left.+\gamma^{2} a_{r, r+1}^{*}(z) a_{s, s+1}^{*}(w)\left(\delta_{s r}-\frac{1}{2}\left(\delta_{s, r-1}+\delta_{s, r+1}\right)\right) \dot{\delta}(w / z)\right) .
\end{aligned}
$$

Fazendo os cálculos acima, o termo em $\delta_{s r}$ torna-se igual a zero. Logo temos termos apenas em $\delta_{s, r-1}$ e $\delta_{s, r+1}$. Assim

$$
\left[\rho_{\mathbb{J}}\left(E_{r}\right)(z), \rho_{\mathbb{J}}\left(E_{s}\right)(w)\right]=0 \text { se }|r-s| \neq 1,
$$

e $\left(R_{5}\right)$ está verificado. Para terminar, temos

$$
\begin{aligned}
{\left[\rho_{\mathbb{J}}\left(F_{r}\right)\left(z_{1}\right), \rho_{\mathbb{J}}\right.} & \left.\left(F_{r}\right)\left(z_{2}\right), \rho_{\mathbb{J}}\left(F_{s}\right)(w)\right]=\left[\rho_{\mathbb{J}}\left(F_{r}\right)\left(z_{1}\right),\left[\rho_{\mathbb{J}}\left(F_{r}\right)\left(z_{2}\right), \rho_{\mathbb{J}}\left(F_{s}\right)(w)\right]\right] \\
= & {\left[-a_{r, r+1}\left(z_{1}\right),\left(a_{r-1, r+1}(w)-\sum_{j=1}^{r-2} a_{j, r+1}(w) a_{j, r-1}^{*}(w)\right) \delta\left(w / z_{2}\right)\right] } \\
& \left.+\left[\sum_{j=1}^{r-1} a_{j, r+1}\left(z_{1}\right) a_{j r}^{*}\left(z_{1}\right), a_{r-1, r+1}(w)-\sum_{l=1}^{r-2} a_{l, r+1}(w) a_{l, r-1}^{*}(w)\right) \delta\left(w / z_{2}\right)\right] \\
= & 0 .
\end{aligned}
$$


De forma análoga, podemos fazer o mesmo para $s=r+1$. Se $s=r-1$, temos

(a) $\left[a_{r, r+1}\left(z_{1}\right) a_{r, r+1}^{*}\left(z_{1}\right)^{2},\left[\rho_{\mathbb{J}}\left(E_{r}\right)\left(z_{2}\right), \rho_{\mathbb{J}}\left(E_{r-1}\right)(w)\right]\right]$

$$
=-a_{r, r+1}(w) a_{r, r+1}^{*}\left(z_{1}\right)^{2} a_{r-1, r+1}^{*}(w) \dot{\delta}\left(z_{1} / z_{2}\right) \delta\left(w / z_{2}\right)
$$

$+\sum_{j=r+2}^{n}\left(a_{r j}\left(z_{2}\right) a_{r j}^{*}\left(z_{2}\right)-a_{r+1, j}\left(z_{2}\right) a_{r+1, j}^{*}\left(z_{2}\right)\right) a_{r, r+1}^{*}\left(z_{1}\right)^{2} a_{r-1, r}^{*}(w) \delta\left(z_{1} / z_{2}\right) \delta\left(w / z_{2}\right)$

$+\sum_{j=r+2}^{n} a_{r j}\left(z_{2}\right) a_{r-1, j}^{*}(w) a_{r, r+1}^{*}\left(z_{1}\right)^{2} \delta\left(z_{1} / z_{2}\right) \delta\left(w / z_{2}\right)$

$+a_{r, r+1}^{*}\left(z_{1}\right)^{2} a_{r-1, r}^{*}(w) \delta\left(z_{1} / z_{2}\right) \delta\left(w / z_{2}\right)\left(\gamma b_{r}\left(z_{2}\right)-\frac{\gamma}{2}\left(b_{r-1}^{*}\left(z_{2}\right)+b_{r+1}^{+}\left(z_{2}\right)\right)\right)$

$-\frac{\gamma^{2}}{2} a_{r, r+1}^{*}\left(z_{1}\right)^{2} a_{r-1, r}^{*}(w) \dot{\delta}\left(w / z_{1}\right) \delta\left(z_{2} / w\right)$.

(b) $\left[\sum_{j=r+2}^{n}\left(a_{r j}\left(z_{1}\right) a_{r j}^{*}\left(z_{1}\right)-a_{r+1, j}\left(z_{1}\right) a_{r+1, j}^{*}\left(z_{1}\right)\right) a_{r, r+1}^{*}\left(z_{1}\right),\left[\rho_{\mathbb{J}}\left(E_{r}\right)\left(z_{2}\right), \rho_{\mathbb{J}}\left(E_{r-1}\right)(w)\right]\right]$

$=-\sum_{j=r+2}^{n}\left(a_{r j}\left(z_{1}\right) a_{r j}^{*}\left(z_{1}\right)-a_{r+1, j}\left(z_{1}\right) a_{r+1, j}^{*}\left(z_{1}\right)\right) a_{r, r+1}^{*}\left(z_{1}\right)^{2} a_{r-1, r}^{*}(w) \delta\left(z_{1} / z_{2}\right) \delta\left(w / z_{2}\right)$

$-\sum_{j=r+2}^{n}\left(a_{r j}\left(z_{1}\right) a_{r j}^{*}\left(z_{1}\right)-a_{r+1, j}\left(z_{1}\right) a_{r+1, j}^{*}\left(z_{1}\right)\right) a_{r, r+1}^{*}\left(z_{1}\right) a_{r-1, r+1}^{*}(w) \delta\left(z_{1} / z_{2}\right) \delta\left(w / z_{2}\right)$

$-2 \sum_{j=r+2}^{n} a_{r+1, j}\left(z_{1}\right) a_{r j}^{*}(w) a_{r, r+1}^{*}\left(z_{1}\right) a_{r-1, r}^{*}(w) \delta\left(z_{1} / z_{2}\right) \delta\left(w / z_{2}\right)$

$-\sum_{j=r+2}^{n} a_{r j}\left(z_{2}\right) a_{r-1, j}^{*}(w) a_{r, r+1}^{*}\left(z_{1}\right)^{2} \delta\left(z_{1} / z_{2}\right) \delta\left(w / z_{2}\right)$

$-\sum_{j=r+2}^{n} a_{r+1, j}\left(z_{1}\right) a_{r-1, j}^{*}(w) a_{r, r+1}^{*}\left(z_{1}\right) \delta\left(w / z_{2}\right) \delta\left(z_{1} / z_{2}\right)$.

(c) $\left[\sum_{j=1}^{r-1} a_{j r}\left(z_{1}\right) a_{j, r+1}^{*}\left(z_{1}\right)-\sum_{j=r+2}^{n} a_{r+1, j}\left(z_{1}\right) a_{r j}^{*}\left(z_{1}\right),\left[\rho_{\mathbb{J}}\left(E_{r}\right)\left(z_{2}\right), \rho_{\mathbb{J}}\left(E_{r-1}\right)(w)\right]\right]$

$=\left(a_{r-1, r+1}^{*}\left(z_{1}\right) a_{r, r+1}^{*}(w) \sum_{j=r+2}^{n}\left(a_{r j}^{*}\left(z_{2}\right) a_{r j}\left(z_{2}\right)-a_{r+1, j}^{*}\left(z_{2}\right) a_{r+1, j}\left(z_{2}\right)\right)\right.$

$-a_{r-1, r+1}^{*}\left(z_{1}\right) a_{r, r+1}^{*}(w)\left(\gamma b_{r}\left(z_{1}\right)-\frac{\gamma}{2}\left(b_{r-1}^{+}\left(z_{1}\right)+b_{r+1}^{+}\left(z_{1}\right)\right)\right)$

$+\frac{\gamma^{2}}{2} a_{r-1, r+1}^{*}\left(z_{1}\right) \dot{a}_{r, r+1}^{*}(w)+2 \sum_{j=r+2}^{n} a_{r+1, j}\left(z_{1}\right) a_{r j}^{*}\left(z_{1}\right) a_{r-1, r}^{*}(w) a_{r, r+1}^{*}(w)$

$\left.+\sum_{j=r+2}^{n} a_{r+1, j}\left(z_{1}\right) a_{r-1, j}^{*}(w) a_{r, r+1}^{*}\left(z_{2}\right)\right) \delta\left(z_{1} / z_{2}\right) \delta\left(w / z_{2}\right)$. 


$$
\begin{aligned}
(d)[- & \gamma a_{r, r+1}^{*}\left(z_{1}\right)\left(b_{r}\left(z_{1}\right)-\frac{1}{2} a_{r, r+1}^{*}\left(z_{1}\right)\left(b_{r-1}^{+}\left(z_{1}\right)+b_{r+1}^{+}\left(z_{1}\right)\right)\right)-\frac{\gamma^{2}}{2} \dot{a}_{r, r+1}^{*}\left(z_{1}\right) \\
& \left.\quad,\left[\rho_{\mathbb{J}}\left(E_{r}\right)\left(z_{2}\right), \rho_{\mathbb{J}}\left(E_{r-1}\right)(w)\right]\right] \\
= & -a_{r, r+1}^{*}\left(z_{2}\right)^{2} a_{r-1, r}^{*}(w) \delta\left(z_{1} / z_{2}\right) \delta\left(w / z_{2}\right)\left(\gamma b_{r}\left(z_{1}\right)-\frac{\gamma}{2}\left(b_{r-1}^{+}\left(z_{1}\right)+b_{r+1}^{+}\left(z_{1}\right)\right)\right) \\
& -\frac{\gamma^{2}}{2} a_{r, r+1}^{*}\left(z_{2}\right)^{2} a_{r-1, r}^{*}(w) \dot{\delta}\left(z_{1} / w\right) \delta\left(w / z_{2}\right) \\
& +a_{r, r+1}^{*}\left(z_{2}\right) a_{r-1, r+1}^{*}(w) \delta\left(z_{1} / z_{2}\right) \delta\left(w / z_{2}\right) \cdot\left(\gamma b_{r}\left(z_{1}\right)-\frac{\gamma}{2}\left(b_{r-1}^{+}\left(z_{1}\right)+b_{r+1}^{+}\left(z_{1}\right)\right)\right) \\
& -\frac{\gamma^{2}}{2} a_{r, r+1}^{*}\left(z_{2}\right) a_{r-1, r+1}^{*}(w) \dot{\delta}\left(z_{1} / z_{2}\right) \delta\left(w / z_{1}\right)-\gamma^{2} a_{r, r+1}^{*}\left(z_{1}\right) a_{r-1, r}^{*}(w) a_{r, r+1}^{*}(w) \delta\left(z_{2} / w\right) \dot{\delta}\left(z_{2} / z_{1}\right) \\
& +\frac{\gamma^{2}}{2} a_{r, r+1}^{*}\left(z_{1}\right) a_{r-1, r+1}^{*}\left(z_{2}\right) \dot{\delta}\left(z_{1} / z_{2}\right) \delta\left(w / z_{2}\right) .
\end{aligned}
$$

Somando as quatro expressões acima, temos

$$
\left[\rho_{\mathbb{J}}\left(E_{r}\right)\left(z_{1}\right), \rho_{\mathbb{J}}\left(E_{r}\right)\left(z_{2}\right), \rho_{\mathbb{J}}\left(E_{r-1}\right)(w)\right]=0
$$

e, de forma análoga, prova-se que

$$
\left[\rho_{\mathbb{J}}\left(E_{r}\right)\left(z_{1}\right), \rho_{\mathbb{J}}\left(E_{r}\right)\left(z_{2}\right), \rho_{\mathbb{J}}\left(E_{r+1}\right)(w)\right]=0
$$

e assim, $\left(R_{6}\right)$ está provada.

Como as relações $\left(R_{1}\right)-\left(R_{6}\right)$ são verdadeiras, o Teorema 2.2.7 está provado. 


\section{Capítulo 3}

\section{Os módulos 『-intermediários de Wakimoto}

Este capítulo tem o objetivo de expor ao leitor os resultados obtidos por B. Cox e V. Futorny em [CF04] e [CF05] para, em seguida, apresentar os obtidos em [Mar12]. A razão para tal escolha é que, em [Mar12], a idéia básica é definir, para $\mathbb{C}[\mathbf{y}]$ e, posteriormente, para $\mathbb{C}[\mathbf{x}, \mathbf{y}]$, operadores $b_{i m}$ 's que não apenas desempenham nos módulos $\mathbb{J}$-intermediários de Wakimoto papéis similares aos dos $b_{i m}$ 's do artigo [CF05] (para os módulos intermediários de Wakimoto) mas que também sejam tais que na hora de se fazer os cálculos, contribuam com "exatamente" os mesmos resultados, tornando a prova da representação por nós obtida completamente análoga à esta.

Por tal razão, a seção 3.1 aborda os conceitos e resultados necessários para o caso dos módulos intermediários de Wakimoto no qual o conceito de produto ordenado normal de duas ou mais distribuições formais é apresentado. Para uma melhor compreensão dos resultados aqui expostos, sugiro ao leitor interessado a leitura de [Kac98]. Posteriormente, apresentamos alguns Lemas auxiliares com o intuito de facilitar os cálculos futuros até finalmente enunciar o Teorema 3.1.27, finalizando sua demonstração através das relações $\left(R_{1}\right)-\left(R_{6}\right)$.

À partir da seção 3.2, nosso objetivo é expor os resultados obtidos em [Mar12]. Para fazer isso, primeiro obtemos um elemento auxiliar $H_{r+1}^{*} \in \widehat{\mathfrak{s l}}(n, \mathbb{C})$ e então, convenientes $b_{i m}$ 's tais que os cálculos subseqüêntes sejam os "mesmos" que os da seção 3.1, facilitando assim nosso trabalho.

É importante frisar que, neste capítulo, alguns conceitos vistos em diferentes tipos de módulos como, por exemplo, $\mathbb{C}[\mathbf{y}]$, diferem "levemente" dependendo do caso abordado, além de não coincidirem necessariamente com os do capítulo 2. Quando tal situação ocorrer, uma observação mencionando explicitamente a diferença entre eles será utilizada.

Com tudo isso em mente, damos início a este capítulo.

\subsection{Os módulos intermediários de Wakimoto}

Fixemos $0 \leq r \leq n, \gamma \in \mathbb{C}^{*}, k=\gamma^{2}-(r+1)$. Seja $H_{i}, E_{i}, F_{i}, i=1, \ldots, n$, um conjunto gerador de $\mathfrak{g}=\mathfrak{s l}(n+1, \mathbb{C})$ e denote

$$
X_{m}=t^{m} \otimes X \text { para } X \in \mathfrak{g} \text { e } m \in \mathbb{Z} .
$$


Seja $\left\{\alpha_{1}, \ldots, \alpha_{n}\right\}$ uma base para $\Delta^{+}$, o conjunto de raízes positivas de $\mathfrak{g}$, tal que $H_{i}=\check{\alpha}_{i}$ e seja $\Delta_{r}$ o sistema de raízes $\left\{\alpha_{1}, \ldots, \alpha_{r}\right\}\left(\Delta_{r}=\emptyset\right.$, se $\left.r=0\right)$ da subálgebra de Lie $\mathfrak{g}_{r}=\mathfrak{s l}(r+1, \mathbb{C})$. Denote por $\mathfrak{h}_{r}$ uma subálgebra de Cartan $\mathfrak{g}_{r}$ gerada por $H_{i}, i=1, \ldots, r$. Seja $\mathfrak{h}_{0}=0$ e

$$
\widetilde{\mathfrak{h}}_{r}=\mathfrak{h}_{r} \oplus \mathbb{C} c \oplus \mathbb{C} d .
$$

Denote por $E_{i m}, F_{i m}, H_{i m}, i=1, \ldots, n, m \in \mathbb{Z}$, os geradores da correspondente álgebra de Loop de $\mathfrak{g}$.

\subsection{1 Álgebra de oscilação}

Seja $\widehat{\mathfrak{a}}$ a álgebra de Heisenberg de dimensão infinita com geradores $a_{i j, m}, a_{i j, m}^{*}$, e $\mathbf{1}$, com $1 \leq i \leq j \leq n$ e $m \in \mathbb{Z}$, munidos das relações

$$
\begin{aligned}
{\left[a_{i j, m}, a_{k l, n}\right] } & =\left[a_{i j, m}^{*}, a_{k l, n}^{*}\right]=0, \\
{\left[a_{i j, m}, a_{k l, n}^{*}\right] } & =\delta_{i k} \delta_{j l} \delta_{m+n, 0} \mathbf{1} \\
{\left[a_{i j, m}, \mathbf{1}\right] } & =\left[a_{i j, m}^{*}, \mathbf{1}\right]=0 .
\end{aligned}
$$

Tal álgebra age em

$$
\mathbb{C}[\mathbf{x}]=\mathbb{C}\left[x_{i j, m} \mid i, j, m \in \mathbb{Z}, 1 \leq i \leq j \leq n\right]
$$

por

$$
\begin{gathered}
a_{i j, m}= \begin{cases}\partial / \partial x_{i j, m} & \text { se } m \geq 0 \text { e } j \leq r, \\
x_{i j, m} & \text { caso contrário. }\end{cases} \\
a_{i j, m}^{*}= \begin{cases}x_{i j,-m} & \text { se } m \leq 0 \text { e } j \leq r, \\
-\partial / \partial x_{i j,-m} & \text { caso contrário. }\end{cases}
\end{gathered}
$$

com $\mathbf{1}$ agindo como identidade. Neste caso, $\mathbb{C}[\mathbf{x}]$ é um $\widehat{\mathfrak{a}}$-módulo gerado por $v$, onde

$$
\begin{gathered}
a_{i j, m} v=0, \text { se } m \geq 0 \text { e } j \leq r, \\
a_{i j, m}^{*} v=0, \text { se } m>0 \text { ou } j>r .
\end{gathered}
$$

Denote por $\widehat{\mathfrak{a}}_{r}$ a subálgebra gerada por $a_{i j, m}$ e $a_{i j, m}^{*}$ e $\mathbf{1}$, onde $1 \leq i \leq j \leq r$ e $m \in \mathbb{Z}$. Se $r=0$, então $\widehat{\mathfrak{a}}_{r}=0$. Seja $A_{n}=\left(\left(\alpha_{i} \mid \alpha_{j}\right)\right)$ a matriz de Cartan de $\mathfrak{s l}(n+1, \mathbb{C})$ e seja $\mathfrak{B}$ a matriz cujas entradas são

$$
\mathfrak{B}_{i j}=\left(\alpha_{i} \mid \alpha_{j}\right)\left(\gamma^{2}-\delta_{i>r} \delta_{j>r}(r+1)+\frac{r}{2} \delta_{i, r+1} \delta_{j, r+1}\right),
$$

onde $\delta_{i>r}= \begin{cases}1 & \text { se } i>r, \\ 0 & \text { caso contrário. }\end{cases}$

Em outras palavras,

$$
\mathfrak{B}=\gamma^{2} A_{n}-(r+1)\left(\begin{array}{cc}
0 & 0 \\
0 & A_{n-r}
\end{array}\right)+r E_{r+1, r+1} .
$$


Também temos a álgebra (de Lie) de Heisenberg $\widehat{\mathfrak{b}}$ com geradores $b_{i m}, 1 \leq i \leq n$, $m \in \mathbb{Z}, \mathbf{1}$, e relações

$$
\left[b_{i m}, b_{j p}\right]=m \mathfrak{B}_{i j} \delta_{m+p, 0} \mathbf{1},\left[b_{i m}, \mathbf{1}\right]=0 .
$$

Para cada $1 \leq i \leq n$, fixe $\lambda_{i} \in \mathbb{C}$ e seja $\lambda=\left(\lambda_{1}, \ldots, \lambda_{n}\right)$. Então a álgebra $\widehat{\mathfrak{b}}$ age no espaço

$$
\mathbb{C}[\mathbf{y}]=\mathbb{C}\left[y_{i m} \mid i, m \in \mathbb{N}^{*}, 1 \leq i \leq n\right]
$$

por

$$
b_{i 0} \mapsto \lambda_{i}, \quad b_{i,-m} \mapsto \mathbf{e}_{i} \cdot \mathbf{y}_{m}, \quad b_{i m} \mapsto m \mathbf{e}_{i} \cdot \frac{\partial}{\partial \mathbf{y}_{m}} \quad \text { para } \quad m>0
$$

e $\mathbf{1} \mapsto 1$. Aqui

$$
\mathbf{y}_{m}=\left(y_{1 m}, \cdots, y_{n m}\right), \quad \frac{\partial}{\partial \mathbf{y}_{m}}=\left(\frac{\partial}{\partial y_{1 m}}, \cdots, \frac{\partial}{\partial y_{n m}}\right)
$$

e $\mathbf{e}_{i}$ são vetores em $\mathbb{C}^{n}$ tais que $\mathbf{e}_{i} \cdot \mathbf{e}_{j}=\mathfrak{B}_{i j}$ onde $\cdot$ é o produto escalar. Como $\mathfrak{B}_{i j}$ é simétrica, ela é ortogonalmente diagonalizável, isto é, existe uma matriz ortogonal $P$ tal que $P^{t} \mathfrak{B} P$ é uma matriz diagonal e então podemos encontrar vetores $\mathbf{e}_{i}$ em $\mathbb{C}^{n}$ com tais propriedades. De fato, para $m \geqslant 0$ e $n<0$, temos

$$
\begin{aligned}
{\left[b_{i m}, b_{j n}\right] } & =\left[m \mathbf{e}_{i} \cdot \frac{\partial}{\partial \mathbf{y}_{m}}, \mathbf{e}_{j} \cdot \mathbf{y}_{-n}\right]=m \sum_{k, l}\left[e_{i k} \frac{\partial}{\partial y_{k m}}, e_{j l} y_{l,-n}\right]=m \delta_{m+n, 0} \sum_{k} e_{i k} e_{j k} \\
& =m \delta_{m+n, 0} \mathfrak{B}_{i j} .
\end{aligned}
$$

Definamos também, para $0 \leqslant r \leqslant n$,

$$
\mathbb{C}_{r}[\mathbf{y}]=\mathbb{C}\left[e_{i} y_{m} \mid i, m \in \mathbb{N}^{*}, 1 \leq i \leq r\right]
$$

onde, se $\mathfrak{B}$ é não-degenerada, temos $\mathbb{C}_{n}[\mathbf{y}]=\mathbb{C}[\mathbf{y}]$ (ver [CF05] para maiores detalhes).

Definição 3.1.6. Para qualquer sequência de elementos $\left\{a_{m}\right\}_{m \in \mathbb{Z}}$ no anel $\operatorname{End}(V), V$ um espaço vetorial, a distribuição formal

$$
a(z)=\sum_{m \in \mathbb{Z}} a_{m} z^{-m-1}
$$

é dita um campo se, para todo $v \in V, a_{m} v=0$ para $m \gg 0$.

Sejam $a^{-}(z)=\sum_{m \geq 0} a_{m} z^{-m-1}$ e $a^{+}(z)=\sum_{m<0} a_{m} z^{-m-1}$. Em particular,

$$
\begin{gathered}
\delta^{-}(z / w)=i_{z, w}\left(\frac{1}{z-w}\right), \\
\delta^{+}(z / w)=-i_{w, z}\left(\frac{1}{z-w}\right) .
\end{gathered}
$$

Observe que

$$
-\partial_{z} \delta(z / w)=\partial_{w} \delta(z-w)=i_{z, w}\left(\frac{1}{(z-w)^{2}}\right)-i_{w, z}\left(\frac{1}{(z-w)^{2}}\right) .
$$


Definição 3.1.7. O produto ordenado normal de duas distribuições formais a $(z)$ e $b(w)$ é definido por

$$
: a(z) b(w):=\sum_{m \in \mathbb{Z}} \sum_{n \in \mathbb{Z}}: a_{m} b_{n}: z^{-m-1} w^{-n-1}
$$

donde segue que:

$$
: a(z) b(w):= \begin{cases}a^{+}(z) b(w)+b(w) a^{-}(z), & \text { se } a(z) \text { e b(z) sãa campos, } \\ a(z) b(w) & \text { se } a(z) \text { não é um campo, } \\ b(w) a(z) & \text { se } a(z) \text { é um campo, mas } b(z) \text { não é. }\end{cases}
$$

Para todo $1 \leq i \leq j \leq n$, defina

$$
\begin{aligned}
a_{i j}^{*}(z) & =\sum_{n \in \mathbb{Z}} a_{i j, n}^{*} z^{-n}, \\
a_{i j}(z) & =\sum_{n \in \mathbb{Z}} a_{i j, n} z^{-n-1}, \\
b_{i}(z) & =\sum_{n \in \mathbb{Z}} b_{i n} z^{-n-1} .
\end{aligned}
$$

Observe que $a_{i j}(z)$ para $j>r$ não é um campo, enquanto que $a_{i j}^{*}(z)$ é. Se $j>r$, chamemos $a_{i j}(z)$ de operador de criação puro e $a_{i j}^{*}(z)$ de aniquilação. Para $j>r$, seja

$$
a_{i j}(z)_{+}=a_{i j}(z), a_{i j}(z)_{-}=0, a_{i j}^{*}(z)_{+}=0 \text { e } a_{i j}^{*}(z)_{-}=a_{i j}^{*}(z) .
$$

Definição 3.1.12. Por indução, definimos o produto ordenado normal de três ou mais distribuições formais da seguinte forma.

$$
: a^{1}\left(z_{1}\right) a^{2}\left(z_{2}\right) \cdots a^{m}\left(z_{m}\right):=: a^{1}\left(z_{1}\right)\left(: a^{2}\left(z_{2}\right) \cdots a^{m}\left(z_{m}\right):\right):
$$

e também

$$
: a(z) b(z):=\lim _{w \rightarrow z}: a(z) b(w):
$$

para apenas alguns pares $a(z)$ e $b(z)$ satisfazendo determinadas propriedades uma vez que nem sempre tal limite está bem-definido. No nosso caso, o produto ordenado

$$
: a_{i j}(z) a_{k l}^{*}(z):=\sum_{m \in \mathbb{Z}}\left(\sum_{n \in \mathbb{Z}}: a_{i j, n} a_{k l, m-n}:\right) z^{-m-1}
$$

está bem-definido como um elemento em $\operatorname{End}(\mathbb{C}[\mathbf{x}] \otimes \mathbb{C}[\mathbf{y}])\left[\left[z, z^{-1}\right]\right]$, para todo $l>r$ (já que $a_{k l, m}^{*}=-\partial / \partial x_{k l,-m}$, para $l>r$ ) ou caso $l \leqslant r$ e $j \leqslant r$.

Sabemos que

$$
\begin{aligned}
{\left[b_{i}(z), b_{j}(w)\right] } & =\sum_{m, n}\left[b_{i, m}, b_{j, n}\right] z^{-m-1} w^{-n-1} \\
& =\sum_{m, n} m \delta_{i j} \delta_{m+n, 0} z^{-m-1} w^{-n-1} \\
& =\sum_{m} m \delta_{i j} z^{-m-1} w^{m-1} \\
& =\mathfrak{B}_{i j} \partial_{w} \delta(z / w)
\end{aligned}
$$


e que

$$
\begin{aligned}
{\left[a_{i j}(z), a_{k l}^{*}(w)\right] } & =\sum_{m, n}\left[a_{\alpha, m}, a_{\beta, n}^{*}\right] z^{-m-1} w^{-n} \\
& =\sum_{m} \delta_{\alpha, \beta} \mathbf{1} z^{-m-1} w^{m} \\
& =\delta_{i k} \delta_{j l} \mathbf{1} \delta(z / w) .
\end{aligned}
$$

Definição 3.1.15. Denote por

$$
\lfloor a b\rfloor=a(z) b(w)-: a(z) b(w):= \begin{cases}{\left[a^{-}(z), b(w],\right.} & \text { se } a(z) \text { e b(z) são campos, } \\ 0 & \text { se a(z) não é um campo, } \\ {[a(z), b(w)]} & \text { se } a(z) \text { é um campo, mas } b(z) \text { não é. }\end{cases}
$$

a contração de duas distribuições formais a $(z)$ e b(w).

Por exemplo, se $j, l \leq r$, então

$$
\begin{aligned}
& \left\lfloor a_{i j} a_{k l}^{*}\right\rfloor=\sum_{m \geq 0} \delta_{i k} \delta_{j l} z^{-m-1} w^{m}=\delta_{i, k} \delta_{j, l} \delta^{-}(z / w)=\delta_{i k} \delta_{j l} i_{z, w}\left(\frac{1}{z-w}\right), \\
& \left\lfloor a_{k l}^{*} a_{i j}\right\rfloor=-\sum_{n<0} \delta_{i k} \delta_{j l} z^{n} w^{-n-1}=-\delta_{i k} \delta_{j l} \delta^{+}(w-z)=\delta_{i k} \delta_{j l} i_{z, w}\left(\frac{1}{w-z}\right) .
\end{aligned}
$$

Teorema 3.1.19 (Teorema de Wick, [Kac98] ). Sejam $a_{i}(z)$ e $b_{j}(z)$ distribuições formais com coeficientes na álgebra associativa $\operatorname{End}(\mathbb{C}[\mathbf{x}] \otimes \mathbb{C}[\mathbf{y}])$, satisfazendo

1. $\left[\left\lfloor a_{i} b_{j}\right\rfloor, c_{k}(z)\right]=0$, para todo $i, j, k$ e $c=$ a ou $c=b$,

2. $\left[a_{i}^{ \pm}(z), b_{j}^{ \pm}(w)\right]=0$ para todo $i$ e $j$.

Então

$$
: a_{1}(z) a_{2}(z) \cdots a_{k}(z): b(w)=\sum_{i=1}^{k}: a_{1}(z) \cdots\left\lfloor a_{i} b\right\rfloor \cdots a_{k}(z):
$$

$e$

$$
\begin{aligned}
& : a_{1}(z) \cdots a_{m}(z):: b_{1}(w) \cdots b_{k}(w):= \\
& \quad=\sum_{s=0}^{\min (m, k)} \sum_{\substack{i_{1}<\cdots<i_{s} \\
j_{1} \neq \cdots \neq j_{s}}}\left\lfloor a_{i_{1}} b_{j_{1}}\right\rfloor \cdots\left\lfloor a_{i_{s}} b_{i_{s}}\right\rfloor: a_{1}(z) \cdots a_{m}(z) b_{1}(w) \cdots b_{k}(w):\left(_{\left(i_{1}, \ldots, i_{s}, j_{1}, \ldots, j_{s}\right)}\right)
\end{aligned}
$$

onde os subscritos $\left(i_{1}, \ldots, i_{s}, j_{1}, \ldots, j_{s}\right)$ significam que tais fatores $a_{i}(z), b_{j}(w)$ com indices $i \in\left\{i_{1}, \ldots, i_{s}\right\}, j \in\left\{j_{1}, \ldots, j_{s}\right\}$ estão omitidos do produto $: a_{1}(z) \cdots a_{m}(z) b_{1}(w) \cdots b_{k}(w)$ :

Teorema 3.1.20 ([Kac98], Teorema 1.4 .3 e 2.3.2). São verdadeiros

(a) Seja a(z) uma distribuição formal. Então na região $|z-w|<|w|$,

$$
a(z)=\sum_{j=0}^{\infty} \partial_{w}^{(j)} a(w)(z-w)^{j}
$$


(b) Sejam $a(z)$ e $b(z)$ distribuições formais com coeficientes na álgebra associativa $\operatorname{End}(\mathbb{C}[\mathbf{x}] \otimes \mathbb{C}[\mathbf{y}])$. São equivalentes:

$$
\begin{aligned}
& \text { 1. }[a(z), b(w)]=\sum_{j=0}^{N-1} \partial_{w}^{(j)} \delta(z / w) c^{j}(w), \text { onde } c^{j}(w) \in \operatorname{End}(\mathbb{C}[\mathbf{x}] \otimes \mathbb{C}[\mathbf{y}])\left[\left[w, w^{-1}\right]\right] . \\
& \text { 2. }\lfloor a b\rfloor=\sum_{j=0}^{N-1} i_{z, w}\left(\frac{1}{(z-w)^{j+1}}\right) c^{j}(w) .
\end{aligned}
$$

Em outras palavras, a parte singular das expansões de produtos de operadores

$$
\lfloor a b\rfloor=\sum_{j=0}^{N-1} i_{z, w}\left(\frac{1}{(z-w)^{j+1}}\right) c^{j}(w)
$$

determinam completamente o colchete das distribuições formais $a(z)$ e $b(w)$. Escrevamos

$$
a(z) b(w) \sim \sum_{j=0}^{N-1} \frac{c^{j}(w)}{(z-w)^{j+1}}
$$

Por exemplo, temos

$$
b_{i}(z) b_{j}(w) \sim \frac{\delta_{i j}}{(z-w)^{2}} .
$$

(para maiores detalhes, ver capítulo 2 de [Kac98])

\subsubsection{Cálculos de campos}

Seja

$\mathcal{H}_{i}(z):=2: a_{i i} a_{i i}^{*}:+\sum_{j=1}^{i-1}\left(: a_{j i} a_{j i}^{*}:-: a_{j, i-1} a_{j, i-1}^{*}:\right)+\sum_{j=i+1}^{n}\left(: a_{i j} a_{i j}^{*}:-: a_{i+1, j} a_{i+1, j}^{*}:\right)$,

onde $a_{i j}$ e $a_{i j}^{*}$ denotam respectivamente $a_{i j}(z)$ e $a_{i j}^{*}(z)$. Para todos $\alpha \in \Delta^{+}$podemos encontrar únicos $1 \leq k \leq l \leq n$ tais que

$$
\alpha=\alpha_{k}+\cdots+\alpha_{l} .
$$

Seja $a_{\alpha}=a_{k l}$ e $a_{\alpha}^{*}=a_{k l}^{*}$. Observe que

$$
\left(\alpha_{i} \mid \alpha\right)=\sum_{j=k}^{l}\left(\alpha_{i} \mid \alpha_{j}\right)=\left(2 \delta_{i k} \delta_{i l}+\delta_{k<i}\left(\delta_{i l}-\delta_{i-1, l}\right)+\delta_{l>i}\left(\delta_{i k}-\delta_{i+1, k}\right)\right) .
$$

Além disso, temos

$$
\begin{aligned}
& \left\lfloor a_{\alpha} a_{\beta}^{*}\right\rfloor= \begin{cases}\delta_{\alpha, \beta} i_{z, w}\left(\frac{1}{z-w}\right) & \text { se } \alpha, \beta \in \Delta_{r}^{+}, \\
0 & \text { caso contrário. }\end{cases} \\
& \left\lfloor a_{\alpha}^{*} a_{\beta}\right\rfloor= \begin{cases}-\delta_{\alpha, \beta} i_{z, w}\left(\frac{1}{z-w}\right) & \text { se } \alpha, \beta \in \Delta_{r}^{+}, \\
-\delta_{\alpha, \beta} \delta(w / z) & \text { caso contrário. }\end{cases}
\end{aligned}
$$


Reescrevamos

$$
\mathcal{H}_{i}(z):=\sum_{\alpha \in \Delta^{+}}\left(\alpha_{i} \mid \alpha\right): a_{\alpha} a_{\alpha}^{*}:
$$

Lema 3.1.24. Para $1 \leqslant i \leqslant j \leqslant n$, com $\alpha, \beta \in \Delta^{+}$, temos as seguintes relações:

$(a)\left[\mathcal{H}_{i}(z), a_{\alpha}(w)\right]=-\delta_{1 \leqslant i \leqslant r} \delta_{\alpha \in \Delta_{r}^{+}}\left(\alpha_{i} \mid \alpha\right) a_{\alpha}(z) \delta(z / w)$,

(b) $\left[\mathcal{H}_{i}(z), a_{\alpha}^{*}(w)\right]=\delta_{1 \leqslant i \leqslant r} \delta_{\alpha \in \Delta_{r}^{+}}\left(\alpha_{i} \mid \alpha\right) a_{\alpha}^{*}(z) \delta(z / w)$,

$(c)\left[\mathcal{H}_{i}(z), \partial_{w} a_{\alpha}^{*}(w)\right]=\delta_{1 \leqslant i \leqslant r} \delta_{\alpha \in \Delta_{r}^{+}}\left(\alpha_{i} \mid \alpha\right) a_{\alpha}^{*}(z) \partial_{w} \delta(z / w)$,

$(d)\left[\mathcal{H}_{i}(z), H_{j}(w)\right]=-\left(\alpha_{i} \mid \alpha_{j}\right)\left(\left(1-\delta_{i>r} \delta_{j>r}\right)(r+1)+\frac{r}{2} \delta_{i, r+1} \delta_{j, r+1}\right) \partial_{w} \delta(z / w)$,

$(e)\left[\mathcal{H}_{i}(z),: a_{\alpha}(w) a_{\beta}(w) a_{\gamma}^{*}(w):\right]=\left(\alpha_{i} \mid \gamma-\alpha+\beta\right): a_{\alpha}(w) a_{\beta}^{*}(w) a_{\gamma}^{*}(w): \delta(z / w)$

$$
-\delta_{\alpha \in \Delta_{r}^{+}}\left(\alpha_{i} \mid \alpha\right)\left(\delta_{\alpha, \beta} a_{\gamma}^{*}(w)+\delta_{\alpha, \gamma} a_{\beta}^{*}(w)\right) \partial_{w} \delta(z / w) .
$$

Demonstração. Pelo Teorema de Wick, temos

$$
\begin{aligned}
\sum_{j}: a_{i j}(z) a_{i j}^{*}(z): a_{k l}(w) & =\sum_{j}\left(: a_{i j}(z)\left\lfloor a_{i j}^{*} a_{k l}\right\rfloor:+:\left\lfloor a_{i j} a_{k l}\right\rfloor a_{i j}^{*}:\right) \\
& =\sum_{j}\left(-\delta_{i k} \delta_{j l}: a_{i j}(z) i_{z, w}\left(\frac{1}{z-w}\right):+0\right) \\
& =\delta_{i k}: a_{k l}(z) i_{z, w}\left(\frac{1}{w-z}\right): \\
& =\delta_{i k} a_{k l}(z) i_{z, w}\left(\frac{1}{w-z}\right) .
\end{aligned}
$$

Como $\alpha=\alpha_{k}+\cdots+\alpha_{l}$, temos

$$
\begin{aligned}
\mathcal{H}_{i}(z) a_{k l}(w)= & \left(2: a_{i i} a_{i i}^{*}:+\sum_{j=1}^{i-1}\left(: a_{j i} a_{j i}^{*}:-: a_{j, i-1} a_{j, i-1}^{*}:\right)\right. \\
& \left.+\sum_{j=i+1}^{n}\left(: a_{i j} a_{i j}^{*}:-: a_{i+1, j} a_{i+1, j}^{*}:\right)\right) a_{k l}(w) \\
\sim & -\delta_{1 \leqslant i, l \leqslant r}\left(2 \delta_{i k} \delta_{i l}+\delta_{k<i}\left(\delta_{i l}-\delta_{i-1, l}\right)+\delta_{l>i}\left(\delta_{i k}-\delta_{i+1, k}\right)\right) a_{k l}(z) i_{z, w}\left(\frac{1}{w-z}\right) \\
& -\delta_{r<l}\left(2 \delta_{i k} \delta_{i l}+\delta_{k<i}\left(\delta_{i l}-\delta_{i-1, l}\right)+\delta_{l>i}\left(\delta_{i k}-\delta_{i+1, k}\right)\right) a_{k l}(z) \delta(z / w) \\
\sim & \delta_{1 \leqslant i, l \leqslant r}\left(\alpha_{i} \mid \alpha\right) a_{k l}(z) i_{z, w}\left(\frac{1}{w-z}\right)-\delta_{r<l}\left(\alpha_{i} \mid \alpha\right) a_{k l}(z) \delta(z / w) .
\end{aligned}
$$

Por outro lado, temos

$$
a_{k l}(w) \mathcal{H}_{i}(z) \sim \delta_{1 \leqslant i, l \leqslant r}\left(\alpha_{i} \mid \alpha\right) a_{k l}(w) i_{w, z}\left(\frac{1}{z-w}\right)
$$

e assim

$$
\begin{aligned}
{\left[\mathcal{H}_{i}(z), a_{\alpha}(w)\right] } & =\delta_{1 \leqslant i, j \leqslant r}\left(\alpha_{i} \mid \alpha\right) a_{k l}(z) \delta(z / w)-\delta_{r<l}\left(\alpha_{i} \mid \alpha\right) a_{k l}(z) \delta(z / w) \\
& =\delta_{1 \leqslant i \leqslant r} \delta_{\alpha \in \Delta_{r}^{*}}\left(\alpha_{i} \mid \alpha\right) a_{\alpha}(z) \delta(z / w),
\end{aligned}
$$

o que prova $(a)$. Cálculos similares provam $(b)$. Para mostrar $(c)$, basta observar que 
$\left[\mathcal{H}_{i}(z), \partial_{w} a_{\alpha}^{*}(w)\right]=\partial_{w}\left(\left[\mathcal{H}_{i}(z), a_{\alpha}^{*}(w)\right]\right)=\delta_{1 \leqslant i \leqslant r} \delta_{\alpha \in \Delta_{r}^{+}}\left(\alpha_{i} \mid \alpha\right) a_{\alpha}^{*}(z) \partial_{w} \delta(z / w)$.

Quanto à $(d)$, temos pelo Teorema de Wick:

$$
\begin{aligned}
: a_{\nu}(z) a_{\mu}^{*}(z):: a_{\alpha}(w) a_{\beta}^{*}(w):= & : a_{\alpha}(w) a_{\beta}^{*}(w) a_{\nu}(z) a_{\mu}^{*}(z):+\left\lfloor a_{\alpha} a_{\beta}^{*}\right\rfloor: a_{\nu}(z) a_{\beta}^{*}: \\
& +\left\lfloor a_{\beta}^{*} a_{\nu}\right\rfloor a_{\alpha}(w) a_{\mu}^{*}(z):+\left\lfloor a_{\alpha} a_{\mu}^{*}\right\rfloor\left\lfloor a_{\beta}^{*} a_{\nu}\right\rfloor .
\end{aligned}
$$

Sendo assim,

$$
\begin{aligned}
& \mathcal{H}_{i}(z) \mathcal{H}_{j}(w)=\sum_{\alpha, \beta \in \Delta^{+}}\left(\alpha_{i} \mid \alpha\right)\left(\alpha_{j} \mid \beta\right): a_{\alpha}(z) a_{\alpha}^{*}(z):: a_{\beta}(w) a_{\beta}^{*}(w): \\
&=\sum_{\alpha, \beta \in \Delta^{+}}\left(\alpha_{i} \mid \alpha\right)\left(\alpha_{j} \mid \beta\right): a_{\alpha}(z) a_{\beta}(w) a_{\alpha}^{*}(z) a_{\beta}^{*}(w):+\sum_{\beta \in \Delta^{+}}\left(\alpha_{i} \mid \beta\right)\left(\alpha_{j} \mid \beta\right): a_{\beta}(w) a_{\beta}^{*}(z):\left\lfloor a_{\beta}^{*} a_{\beta}\right\rfloor \\
& \quad+\sum_{\alpha \in \Delta^{+}}\left(\alpha_{i} \mid \alpha\right)\left(\alpha_{j} \mid \alpha\right): a_{\alpha}(z) a_{\alpha}^{*}(w):\left\lfloor a_{\alpha}^{*} a_{\alpha}\right\rfloor+\sum_{\alpha \in \Delta^{+}}\left(\alpha_{i} \mid \alpha\right)\left(\alpha_{j} \mid \alpha\right)\left\lfloor a_{\alpha}^{*} a_{\alpha}\right\rfloor\left\lfloor a_{\alpha} a_{\alpha}^{*}\right\rfloor \\
&= \sum_{\alpha, \beta \in \Delta^{+}}\left(\alpha_{i} \mid \alpha\right)\left(\alpha_{j} \mid \beta\right): a_{\alpha}(z) a_{\beta}(w) a_{\alpha}^{*}(z) a_{\beta}^{*}(w):+\sum_{\beta \in \Delta^{+}}\left(\alpha_{i} \mid \beta\right)\left(\alpha_{j} \mid \beta\right): a_{\beta}(w) a_{\beta}^{*}(z):\left\lfloor a_{\beta}^{*} a_{\beta}\right\rfloor \\
&+\sum_{\alpha \in \Delta^{+}}\left(\alpha_{i} \mid \alpha\right)\left(\alpha_{j} \mid \alpha\right): a_{\alpha}(z) a_{\alpha}^{*}(w):\left\lfloor a_{\alpha}^{*} a_{\alpha}\right\rfloor \\
&+\sum_{\alpha \in \Delta^{+}}\left(\alpha_{i} \mid \alpha\right)\left(\alpha_{j} \mid \alpha\right) i_{z, w}\left(\frac{1}{z-w}\right)\left(-i_{z, w}\left(\frac{1}{w-z}\right)\right) \\
&= \sum_{\alpha, \beta \in \Delta^{+}}\left(\alpha_{i} \mid \alpha\right)\left(\alpha_{j} \mid \beta\right): a_{\alpha}(z) a_{\beta}(w) a_{\alpha}^{*}(z) a_{\beta}^{*}(w):+\sum_{\beta \in \Delta^{+}}\left(\alpha_{i} \mid \beta\right)\left(\alpha_{j} \mid \beta\right): a_{\beta}(w) a_{\beta}^{*}(z):\left\lfloor a_{\beta}^{*} a_{\beta}\right\rfloor \\
&+\sum_{\alpha \in \Delta^{+}}\left(\alpha_{i} \mid \alpha\right)\left(\alpha_{j} \mid \alpha\right): a_{\alpha}(z) a_{\alpha}^{*}(w):\left\lfloor a_{\alpha}^{*} a_{\alpha}\right\rfloor+\sum_{\alpha \in \Delta^{+}}\left(\alpha_{i} \mid \alpha\right)\left(\alpha_{j} \mid \alpha\right) i_{w, z}\left(\frac{1}{(z-w)^{2}}\right) .
\end{aligned}
$$

Logo ao considerarmos $\left[\mathfrak{h}_{i}(z), \mathfrak{h}_{j}(w)\right]$, teremos que as três primeiras parcelas se cancelam sobrando apenas a última, o que nos dá

$$
\begin{aligned}
{\left[\mathcal{H}_{i}(z), \mathcal{H}_{j}(w)\right] } & =\sum_{\alpha \in \Delta^{+}}\left(\alpha_{i} \mid \alpha\right)\left(\alpha_{j} \mid \alpha\right)\left(i_{w, z}\left(\frac{1}{(z-w)^{2}}\right)-i_{z, w}\left(\frac{1}{(z-w)^{2}}\right)\right) \\
& =-\left(\alpha_{i} \mid \alpha_{j}\right)\left(\left(1-\delta_{i>r} \delta_{j>r}\right)(r+1)+\frac{r}{2} \delta_{i, r+1} \delta_{j, r+1}\right) \partial_{w} \delta(z / w)
\end{aligned}
$$

já que, para $j \leqslant r$, temos

$$
\sum_{\alpha \in \Delta_{r}^{+}}\left(\alpha_{j} \mid \alpha\right) \alpha=(r+1) \alpha_{j}, \text { e } \sum_{\alpha \in \Delta_{r}^{+}}\left(\alpha_{r+1} \mid \alpha\right)^{2}=r .
$$

Finalmente, provemos $(e)$ :

$$
\begin{aligned}
: a_{\nu}(z) & a_{\nu}^{*}(z):: a_{\alpha}(w) a_{\beta}^{*}(w) a_{\gamma}^{*}(w):=: a_{\nu}(z) a_{\nu}^{*}(z) a_{\alpha}(w) a_{\beta}^{*}(w) a_{\gamma}^{*}(w): \\
& +\left\lfloor a_{\nu}^{*} a_{\alpha}\right\rfloor: a_{\nu}(z) a_{\beta}^{*}(w):+\left\lfloor a_{\nu} a_{\beta}^{*}\right\rfloor: a_{\alpha}(w) a_{\nu}^{*}(z) a_{\gamma}^{*}(w): \\
& +\left\lfloor a_{\nu} a_{\gamma}^{*}\right\rfloor: a_{\alpha}(w) a_{\beta}^{*}(z) a_{\gamma}^{*}(z):+\left\lfloor a_{\nu}^{*} a_{\alpha}\right\rfloor\left\lfloor a_{\nu} a_{\beta}^{*}\right\rfloor a_{\gamma}^{*}(w)+\left\lfloor a_{\nu}^{*} a_{\alpha}\right\rfloor\left\lfloor a_{\nu} a_{\gamma}^{*}\right\rfloor a_{\beta}^{*}(w) .
\end{aligned}
$$

Desta forma, 


$$
\begin{aligned}
& \mathcal{H}_{i}(z): a_{\alpha}(w) a_{\beta}^{*}(w) a_{\gamma}^{*}(w):=\sum_{\nu \in \Delta^{+}}\left(\alpha_{i} \mid \nu\right): a_{\nu}(z) a_{\nu}^{*}(z):: a_{\alpha}(w) a_{\beta}^{*}(w) a_{\gamma}^{*}(w): \\
& \sim \sum_{\nu \in \Delta^{+}}\left(\alpha_{i} \mid \nu\right)\left(\left(\left\lfloor a_{\nu}^{*} a_{\alpha}\right\rfloor: a_{\nu}(z) a_{\beta}^{*}(w) a_{\gamma}^{*}(w):+\left\lfloor a_{\nu} a_{\beta}^{*}\right\rfloor: a_{\alpha}(w) a_{\nu}^{*}(z) a_{\gamma}^{*}(w):\right.\right. \\
& \left.\left.\quad+\left\lfloor a_{\nu} a_{\gamma}^{*}\right\rfloor: a_{\alpha}(w) a_{\beta}^{*}(z) a_{\nu}^{*}(z):\right)+\left(\left\lfloor a_{\nu}^{*} a_{\alpha}\right\rfloor\left\lfloor a_{\nu} a_{\beta}^{*}\right\rfloor a_{\gamma}^{*}(w)+\left\lfloor a_{\nu}^{*} a_{\alpha}\right\rfloor\left\lfloor a_{\nu} a_{\gamma}^{*}\right\rfloor a_{\beta}^{*}(w)\right)\right) \\
& \quad \sim\left(\left(\alpha_{i} \mid \alpha\right)\left\lfloor a_{\alpha}^{*} a_{\alpha}\right\rfloor: a_{\alpha}(z) a_{\beta}^{*}(w) a_{\gamma}^{*}(w):+\left(\alpha_{i} \mid \beta\right)\left\lfloor a_{\beta} a_{\beta}^{*}\right\rfloor: a_{\alpha}(w) a_{\beta}^{*}(z) a_{\gamma}^{*}(w):\right. \\
& \left.\quad+\left(\alpha_{i} \mid \gamma\right)\left\lfloor a_{\gamma} a_{\gamma}^{*}\right\rfloor: a_{\alpha}(z) a_{\beta}^{*}(z) a_{\mu}^{*}(w)\right):+\left(\alpha_{i} \mid \alpha\right)\left(\delta_{\alpha, \beta} a_{\gamma}^{*}(w)+\delta_{\alpha, \gamma} a_{\beta}^{*}(w)\right)\left\lfloor a_{\alpha}^{*} a_{\alpha}\right\rfloor\left\lfloor a_{\alpha} a_{\alpha}^{*}\right\rfloor,
\end{aligned}
$$

o que nós dá o resultado desejado.

Lema 3.1.25. São verdadeiras as relações

(1) $\left[a_{i j}(z), a_{k l}^{*}(w)\right]=\delta_{i k} \delta_{j l} \delta(z / w)$,

(2) $\left[a_{i j}(z) a_{i j}^{*}(z), a_{i j}(w) a_{i j}^{*}(w)\right]=\delta_{1 \leqslant i, j \leqslant r} \partial_{w} \delta(z / w)$,

(3) $\left[a_{i j}(z), \partial_{w} a_{k l}^{*}(w)\right]=\delta_{i k} \delta_{j l} \partial_{w} \delta(z / w)$,

(4) $\partial_{w} a_{i j}^{*}(w) \delta(z / w)=a_{i j}^{*}(z) \partial_{w} \delta(z / w)-a_{i j}^{*}(w) \partial_{w} \delta(z / w)$.

Demonstração. Temos

(1) $\left[a_{i j}(z), a_{k l}^{*}(w)\right]=\sum_{n, m \in \mathbb{Z}}\left[a_{i j, n}, a_{k l, m}\right] z^{-n-1} w^{-m}=\sum_{n, m \in \mathbb{Z}} \delta_{i k} \delta_{j l} \delta_{m,-n} z^{-n-1} w^{-m}$

$$
=\sum_{n \in \mathbb{Z}} \delta_{i k} \delta_{j l} z^{-n-1} w^{n}=\delta_{i k} \delta_{j l} \delta(z / w) .
$$

(2) $\left[a_{i j}(z) a_{i j}^{*}(z), a_{i j}(w) a_{i j}^{*}(w)\right]$

$$
\begin{aligned}
& =a_{i j}(z)\left[a_{i j}^{*}(z), a_{i j}(w)\right] a_{i j}^{*}(w)+a_{i j}(w)\left[a_{i j}(z), a_{i j}^{*}(w)\right] a_{i j}^{*}(z) \\
& =-a_{i j}(z) \delta(z / w) a_{i j}^{*}(w)+a_{i j}(w) \delta(z / w) a_{i j}^{*}(z) \\
& =\delta_{1 \leqslant i, j \leqslant r} \partial_{w} \delta(z / w) .
\end{aligned}
$$

Derivando a expressão (1) em $w$, temos

(3) $\delta_{i k} \delta_{j l} \partial_{w} \delta(z / w)=\partial_{w}\left(\left[a_{i j}(z), a_{k l}^{*}(w)\right]\right)=\left[a_{i j}(z), \partial_{w} a_{k l}^{*}(w)\right]$

e, para finalizar,

(4) $a_{i j}^{*}(z) \partial_{w} \delta(z / w)=\partial_{w}\left(a_{i j}^{*}(z) \delta(z / w)\right)=\partial_{w}\left(a_{i j}^{*}(w) \delta(z / w)\right)$

$$
=\partial_{w} a_{i j}^{*}(w) \delta(z / w)+a_{i j}^{*}(w) \partial_{w} \delta(z / w) .
$$

Assim,

$$
\partial_{w} a_{i j}^{*}(w) \delta(z / w)=a_{i j}^{*}(z) \partial_{w} \delta(z / w)-a_{i j}^{*}(w) \partial_{w} \delta(z / w),
$$

o que prova o Lema. 
Lema 3.1.26. São verdadeiras as relações

(a) $\sum_{k=i+1}^{n} \sum_{l=j+1}^{n}\left[a_{i k}(z) a_{i+1, k}^{*}(z), a_{j l}(w) a_{j+1, l}^{*}(w)\right]$

$$
=\left(\delta_{i, j+1} \sum_{k=j+2}^{n} a_{j k}(z) a_{j+2, k}^{*}(z)-\delta_{j, i+1} \sum_{k=i+2}^{n} a_{i k}(z) a_{i+2, k}^{*}(z)\right) \delta(z / w),
$$

(b) $\sum_{k=1}^{i-1} \sum_{l=j+1}^{n}\left[a_{k, i-1}(z) a_{k i}^{*}(z), a_{j l}(w) a_{j+1, l}^{*}(w)\right]=-\delta_{j, i-1} a_{i-1, i-1}(z) a_{i i}^{*}(z) \delta(z / w)$,

(c) $\sum_{k=1}^{i-1} \sum_{l=j+1}^{n}\left[: a_{i i}^{*} a_{k, i-1} a_{k, i-1}^{*}:, a_{j l}(w) a_{j+1, l}^{*}(w)\right]$

$$
-\sum_{k=1}^{i} \sum_{l=j+1}^{n}\left[: a_{i i}^{*} a_{k i} a_{k i}^{*}:, a_{j l}(w) a_{j+1, l}^{*}(w)\right]=0
$$

(d) $\sum_{k=1}^{i-1}\left[: a_{i i}^{*} a_{k, i-1} a_{k, i-1}^{*}:, a_{j j}(w)\right]-\sum_{k=1}^{i}\left[: a_{i i}^{*} a_{k i} a_{k i}^{*}:, a_{j j}(w)\right]=$

$$
\begin{aligned}
& -\delta_{i j}\left(\sum_{k=1}^{i-1}: a_{k, i-1}(z) a_{k, i-1}^{*}(z):-\sum_{k=1}^{i}: a_{k i}(z) a_{k i}^{*}(z):\right) \delta(z / w) \\
& -\left(\delta_{j, i-1} a_{i-1, i-1}(z) a_{i i}^{*}(z)-\delta_{i j}: a_{i i}(z) a_{i i}^{*}(z):\right) \delta(z / w),
\end{aligned}
$$

(e) $\left[\sum_{k=1}^{i-1}: a_{i i}^{*} a_{k, i-1} a_{k, i-1}^{*}:, \sum_{l=1}^{j-1}: a_{j j}^{*} a_{l, j-1} a_{l, j-1}^{*}:\right]$

$$
\begin{aligned}
= & \delta_{j, i-1} \sum_{k=1}^{i-2}: a_{i i}^{*}(z) a_{i-1, i-1}^{*}(z) a_{k, i-2}(w) a_{k, i-2}^{*}(w): \delta(z / w) \\
& -\delta_{i, j-1} \sum_{l=1}^{j-2}: a_{j j}^{*}(w) a_{j-1, j-1}^{*}(w) a_{l, j-2}(z) a_{l, j-2}^{*}(z): \delta(z / w) \\
& -(i-1) \delta_{1 \leqslant i-1 \leqslant r} \delta_{i j}: a_{i i}^{*}(z) a_{i i}^{*}(w): \partial_{w} \delta(z / w),
\end{aligned}
$$

$(f)\left[\sum_{k=1}^{i-1}: a_{i i}^{*} a_{k, i-1} a_{k, i-1}^{*}:, \sum_{l=1}^{j}: a_{j j}^{*} a_{l j} a_{l j}^{*}:\right]$

$$
\begin{aligned}
= & \delta_{j, i-1} \sum_{k=1}^{i-1}: a_{i i}^{*}(z) a_{i-1, i-1}^{*}(z) a_{k, i-1}(w) a_{k, i-1}^{*}(w): \delta(z / w) \\
& -\delta_{i j} \sum_{l=1}^{i-1}: a_{i i}^{*}(w) a_{i i}^{*}(w) a_{l, i-1}(z) a_{l, i-1}^{*}(z): \delta(z / w) \\
& -i \delta_{1 \leqslant i-1 \leqslant r} \delta_{j, i-1}: a_{i i}^{*}(z) a_{i-1, i-1}^{*}(w): \partial_{w} \delta(z / w),
\end{aligned}
$$

(g) $\left[\sum_{k=1}^{i-1}: a_{i i}^{*} a_{k i} a_{k i}^{*}:, \sum_{l=1}^{j}: a_{j j}^{*} a_{l j} a_{l j}^{*}:\right]$

$$
=-(3+i) \delta_{1 \leqslant i \leqslant r} \delta_{i j}: a_{i i}^{*}(z) a_{i i}^{*}(w): \partial_{w} \delta(z / w),
$$




$$
\begin{aligned}
& \text { (h) } \sum_{l=j+1}^{n}\left(\sum_{k=1}^{i-1}\left[: a_{i i}^{*} a_{k, i-1} a_{k, i-1}^{*}:, a_{j+1, l}(w) a_{j l}^{*}(w)\right]-\sum_{k=1}^{i}\left[: a_{i i}^{*} a_{k i} a_{k i}^{*}:, a_{j+1, l}(w) a_{j l}^{*}(w)\right]\right) \\
& =-\delta_{j, i-1}\left(\sum_{k=1}^{i-1}: a_{i-1, i}^{*}(w) a_{k, i-1}(z) a_{k, i-1}^{*}(z):-\sum_{k=1}^{i}: a_{i-1, i}^{*}(w) a_{k i}(z) a_{k i}^{*}(z):\right) \delta(z / w) \\
& +\delta_{i \leqslant r} \delta_{j, i-1} a_{i-1, i}^{*}(z) \partial_{w} \delta(z / w), \\
& \text { (i) } \sum_{k=1}^{i-1}\left[: a_{i i}^{*} a_{k, i-1} a_{k, i-1}^{*}:, \sum_{l=1}^{j-1} a_{l, j-1} a_{l j}^{*}\right]-\sum_{k=1}^{i}\left[: a_{i i}^{*} a_{k i} a_{k i}^{*}:, \sum_{l=1}^{j-1} a_{l, j-1} a_{l j}^{*}\right] \\
& =\left(\delta_{j, i-1} \sum_{l=1}^{i-2}: a_{i i}^{*} a_{l, i-2} a_{l, i-1}^{*}:-2 \delta_{i j} \sum_{l=1}^{i-1}: a_{i i}^{*} a_{l, i-1} a_{l i}^{*}:+\delta_{i, j-1} \sum_{l=1}^{i}: a_{i i}^{*} a_{l i} a_{l, i+1}^{*}:\right. \\
& \left.-\delta_{i, j-1}\left(\sum_{l=1}^{i-1}: a_{i, i+1}^{*} a_{l, i-1} a_{l, i-1}^{*}:-\sum_{l=1}^{i}: a_{i, i+1}^{*} a_{l i} a_{l i}^{*}:\right)\right) \delta(z / w), \\
& (j)\left[\sum_{k=1}^{i-1} a_{k, i-1} a_{k i}^{*}, \sum_{l=1}^{j-1} a_{l, j-1} a_{l j}^{*}\right]=\left(\delta_{j, i-1} \sum_{l=1}^{i-2}: a_{l, i-2} a_{l i}^{*}:-\delta_{i, j-1} \sum_{l=1}^{j-2}: a_{l, j-2} a_{l j}^{*}:\right) \delta(z / w), \\
& (k)\left[\sum_{k=i+1}^{n} a_{i+1, k}(z) a_{i k}^{*}(z), \sum_{l=j+1}^{n} a_{j+1, l}(w) a_{j l}^{*}(w)\right] \\
& =\left(\delta_{i, j-1} \sum_{l=i+2}^{n}: a_{i+2, l} a_{i l}^{*}:-\delta_{j, i-1} \sum_{l=j+2}^{n}: a_{j+2, k} a_{j k}^{*}:\right) \delta(z / w), \\
& (l)\left[\sum_{k=1}^{i-1} a_{k, i-1}(z) a_{k i}^{*}(z), \sum_{l=j+1}^{n} a_{j+1, l}(w) a_{j l}^{*}(w)\right]=0 .
\end{aligned}
$$

Demonstração. Temos

$$
\text { (a) } \begin{aligned}
& \sum_{k=i+1}^{n} \sum_{l=j+1}^{n}\left[a_{i k}(z) a_{i+1, k}^{*}(z), a_{j l}(w) a_{j+1, l}^{*}(w)\right] \\
= & \sum_{k=i+1}^{n} \sum_{l=j+1}^{n}\left(a_{j l}(w)\left[a_{i k}(z), a_{j+1, l}^{*}(w)\right] a_{i+1, k}^{*}(z)+a_{i k}(z)\left[a_{i+1, k}^{*}(z), a_{j l}(w)\right] a_{j+1, l}^{*}(w)\right) \\
= & \sum_{k=i+1}^{n} \sum_{l=j+1}^{n}\left(a_{j l}(w) \delta_{i, j+1} \delta_{k l} \delta(z / w) a_{i+1, k}^{*}(z)+a_{i k}(z)\left(-\delta_{j, i+1} \delta_{k l} \delta(z / w)\right) a_{j+1, l}^{*}(w)\right) \\
= & \delta_{i, j+1}\left(\sum_{k=j+2}^{n} a_{j k}(w) \delta(z / w) a_{j+2, k}^{*}(z)\right)-\delta_{j, i+1}\left(\sum_{i+2}^{n} a_{i k}(z) \delta(z / w)\right) a_{i+2, k}^{*}(w) \\
= & \left(\delta_{i, j+1} \sum_{k=j+2}^{n} a_{j k}(z) a_{j+2, k}^{*}(z)-\delta_{j, i+1} \sum_{k=i+2}^{n} a_{i k}(z) a_{i+2, k}^{*}(z)\right) \delta(z / w) .
\end{aligned}
$$




$$
\begin{aligned}
\text { (b) } & \sum_{k=1}^{i-1} \sum_{l=j+1}^{n}\left[a_{k, i-1}(z) a_{k i}^{*}(z), a_{j l}(w) a_{j+1, l}^{*}(w)\right] \\
= & \sum_{k=1}^{i-1} \sum_{l=j+1}^{n}\left(a_{k, i-1}(z)\left[a_{k i}^{*}(z), a_{j l}(w)\right] a_{j+1, l}^{*}(w)+a_{j l}(w)\left[a_{k, i-1}(z), a_{j+1, l}^{*}(w)\right] a_{k i}^{*}(z)\right) \\
= & \sum_{k=1}^{i-1} \sum_{l=j+1}^{n}\left(a_{k, i-1}(z)\left(-\delta_{k j} \delta_{i l} \delta(z / w)\right) a_{j+1, l}^{*}(w)+a_{j l}(w) \delta_{k, j+1} \delta_{i-1, l} \delta(z / w) a_{k i}^{*}(z)\right) \\
= & -\delta_{j, i-1} a_{i-1, i-1}(z) a_{i i}^{*}(z) \delta(z / w) . \\
(c) & \sum_{l=j+1}^{n}\left[\sum_{k=1}^{i-1}: a_{i i}^{*} a_{k, i-1} a_{k, i-1}^{*}:-\sum_{k=1}^{i}: a_{i i}^{*} a_{k i} a_{k i}^{*}:, a_{j l}(w) a_{j+1, l}^{*}(w)\right] \\
= & \sum_{l=j+1}^{n} \sum_{k=1}^{i-1}\left[: a_{i i}^{*} a_{k, i-1} a_{k, i-1}^{*}:, a_{j l} a_{j+1, l}^{*}\right]-\sum_{l=j+1}^{n} \sum_{k=1}^{i}\left[: a_{i i}^{*} a_{k i} a_{k i}^{*}:, a_{j l} a_{j+1, l}^{*}\right] \\
= & \delta_{i \geq j+1} \delta_{i j} \sum_{k=1}^{i-1}: a_{k, i-1} a_{k, i-1}^{*} a_{i+1, i}^{*}: \delta(z / w)-\delta_{j+1 \leq i-1}: a_{j, i-1} a_{i i}^{*} a_{j+1, i-1}^{*}: \delta(z / w) \\
& \quad+\delta_{j+1 \leq i-1}: a_{j, i-1} a_{i i}^{*} a_{j+1, i-1}^{*}: \delta(z / w)+\delta_{i j} \delta_{i \geq j+1} \sum_{k=1}^{i}: a_{k i} a_{k i}^{*} a_{j+1, l}^{*}: \delta(z / w) \\
& \quad+\delta_{j \leq i-1}: a_{j i} a_{i i}^{*} a_{j+1, i}^{*}: \delta(z / w)-\delta_{j \leq i-1}: a_{j i} a_{i i}^{*} a_{j+1, i}^{*}: \delta(z / w) \\
= & 0 .
\end{aligned}
$$

Para $(d)$, temos

$$
\begin{aligned}
& \sum_{k=1}^{i-1}: a_{i i}^{*}(z) a_{k, i-1}(z) a_{k, i-1}^{*}(z): a_{j j}(w)-\sum_{k=1}^{i}: a_{i i}^{*}(z) a_{k i}(z) a_{k i}^{*}(z): a_{j j}(w) \\
& =\sum_{k=1}^{i-1}:\left\lfloor a_{i i}^{*} a_{j j}\right\rfloor a_{k, i-1}(z) a_{k, i-1}^{*}(z):+\sum_{k=1}^{i}:\left\lfloor a_{i i}^{*} a_{j j}\right\rfloor a_{k i}(z) a_{k i}^{*}(z): \\
& \quad+\sum_{k=1}^{i-1}: a_{i i}^{*}(z) a_{k, i-1}(z)\left\lfloor a_{k, i-1}^{*} a_{j j}\right\rfloor:-\sum_{k=1}^{i}: a_{i i}^{*}(z) a_{k, i}(z)\left\lfloor a_{k i}^{*} a_{j j}\right\rfloor: \\
& =\sum_{k=1}^{i-1} \delta_{i j} i_{z, w}\left(\frac{1}{z-w}\right): a_{k, i-1}(z) a_{k, i-1}^{*}(z):-\sum_{k=1}^{i} \delta_{i j} i_{z, w}\left(\frac{1}{z-w}\right): a_{k i}(z) a_{k i}^{*}(z): \\
& \quad+\delta_{j k} \delta_{j, i-1} i_{z, w}\left(\frac{1}{z-w}\right): a_{i i}^{*}(z) a_{i-1, i-1}(z):-\delta_{j k} \delta_{j i} i_{z, w}\left(\frac{1}{z-w}\right): a_{i i}^{*}(z) a_{i i}(z): .
\end{aligned}
$$

De forma análoga, fazemos os cálculos para

$$
a_{j j}(w)\left(\sum_{k=1}^{i-1}: a_{i i}^{*}(z) a_{k, i-1}(z) a_{k, i-1}^{*}(z): a_{j j}(w)-\sum_{k=1}^{i}: a_{i i}^{*}(z) a_{k i}(z) a_{k i}^{*}(z):\right)
$$

obtendo $(d)$. 
Pelo Teorema de Wick, temos

$$
\begin{aligned}
\left(\sum_{k=1}^{i-1}: a_{i i}^{*} a_{k, i-1} a_{k, i-1}^{*}:\right)\left(\sum_{l=1}^{j-1}: a_{j j}^{*} a_{l, j-1} a_{l, j-1}^{*}:\right) & \\
\sim & \delta_{j, i-1} \sum_{k=1}^{i-2}: a_{i i}^{*}(z) a_{i-1, i-1}^{*}(z) a_{k, i-2}(w) a_{k, i-2}^{*}(w):\left\lfloor a_{i-1, i-1} a_{j j}^{*}\right\rfloor \\
& +\delta_{i j} \sum_{l=1}^{i-1}: a_{i i}^{*}(z) a_{i i}^{*}(w) a_{l, i-1}(w) a_{l, i-1}^{*}(z):\left\lfloor a_{i-1, i-1} a_{j-1, j-1}^{*}\right\rfloor \\
& +\delta_{i j} \sum_{l=1}^{i-1}: a_{i i}^{*}(z) a_{i i}^{*}(w) a_{l, i-1}(z) a_{l, i-1}^{*}(w):\left\lfloor a_{i-1, i-1}^{*} a_{j-1, j-1}\right\rfloor \\
& +\delta_{i, j-1} \sum_{l=1}^{j-2}: a_{j j}^{*}(w) a_{j-1, j-1}^{*}(w) a_{l, j-2}(z) a_{l, j-2}^{*}(z):\left\lfloor a_{i, i}^{*} a_{j-1, j-1}\right\rfloor \\
& +\delta_{i j}: a_{i i}^{*}(z) a_{i i}^{*}(w):\left(\sum_{l=1}^{i-1}\left\lfloor a_{l, i-1} a_{l, j-1}^{*}\right\rfloor\left\lfloor a_{l, i-1}^{*} a_{l, j-1}\right\rfloor\right) .
\end{aligned}
$$

Portanto

$$
\begin{aligned}
(e)\left[\sum_{k=1}^{i-1}: a_{i i}^{*} a_{k, i-1} a_{k, i-1}^{*}:, \sum_{l=1}^{j-1}: a_{j j}^{*} a_{l, j-1} a_{l, j-1}^{*}:\right] \\
=\delta_{j, i-1} \sum_{k=1}^{i-2}: a_{i i}^{*}(z) a_{i-1, i-1}^{*}(z) a_{k, i-2}(w) a_{k, i-2}^{*}(w): \delta(z / w) \\
\\
+\delta_{i, j-1} \sum_{l=1}^{j-2}: a_{j j}^{*}(w) a_{j-1, j-1}^{*}(w) a_{l, j-2}(z) a_{l, j-2}^{*}(z): \delta(z / w) \\
\\
-(i-1) \delta_{1 \leq i-1 \leq r} \delta_{i j}: a_{i i}^{*}(z) a_{i i}^{*}(w): \partial_{w} \delta(z / w) .
\end{aligned}
$$

Novamente pelo Teorema de Wick

$$
\begin{aligned}
& \left(\sum_{k=1}^{i}: a_{i i}^{*} a_{k i} a_{k i}^{*}:\right)\left(\sum_{l=1}^{j}: a_{j j}^{*} a_{l j} a_{l j}^{*}:\right) \sim \delta_{i j}: a_{i i}^{*}(z)^{2}\left(\sum_{k=1}^{i} a_{k i}(w) a_{k i}^{*}(w)\right):\left\lfloor a_{i i} a_{j j}^{*}\right\rfloor \\
& \quad+\delta_{i j} \sum_{l=1}^{i}: a_{i i}^{*}(z) a_{i i}^{*}(w) a_{l i}(w) a_{l i}^{*}(z):\left\lfloor a_{i i} a_{j j}^{*}\right\rfloor+\delta_{i j} \sum_{l=1}^{i}: a_{i i}^{*}(w) a_{i i}^{*}(w) a_{l i}(z) a_{l i}^{*}(z):\left\lfloor a_{i i}^{*} a_{j j}\right\rfloor \\
& \quad+\delta_{i j} \sum_{l=1}^{i}: a_{i i}^{*}(z) a_{i i}^{*}(w) a_{l i}(z) a_{l i}^{*}(w):\left\lfloor a_{i i}^{*} a_{j j}\right\rfloor+3 \delta_{i j}: a_{i i}^{*}(z) a_{i i}^{*}(w):\left\lfloor a_{i i} a_{j j}^{*}\right\rfloor\left\lfloor a_{i i}^{*} a_{j j}\right\rfloor \\
& \quad+\delta_{i j}: a_{i i}^{*}(z) a_{i i}^{*}(w):\left(\sum_{l=1}^{i}\left\lfloor a_{l i} a_{l j}^{*}\right\rfloor\left\lfloor a_{l i}^{*} a_{l j}\right\rfloor\right)
\end{aligned}
$$




$$
\begin{aligned}
&\left(\sum_{k=1}^{i-1}: a_{i i}^{*} a_{k, i-1} a_{k, i-1}^{*}:\right)\left(\sum_{l=1}^{j}: a_{j j}^{*} a_{l j} a_{l j}^{*}:\right) \\
& \sim \delta_{j, i-1} \sum_{k=1}^{i-1}: a_{i i}^{*}(z) a_{i-1, i-1}^{*}(z) a_{k, i-1}(w) a_{k, i-1}^{*}(w):\left\lfloor a_{i-1, i-1} a_{j j}^{*}\right\rfloor \\
& \quad+\delta_{j, i-1} \sum_{l=1}^{i-1}: a_{i i}^{*}(z) a_{i-1, i-1}^{*}(w) a_{l, i-1}(w) a_{l, i-1}^{*}(z):\left\lfloor a_{i-1, i-1} a_{j, i}^{*}\right\rfloor \\
&+\delta_{i j} \sum_{l=1}^{i-1}: a_{i i}^{*}(w) a_{i i}^{*}(w) a_{l, i-1}(z) a_{l, i-1}^{*}(z):\left\lfloor a_{i-1, i-1}^{*} a_{j-1, j-1}\right\rfloor \\
&+\delta_{j, i-1} \sum_{l=1}^{i-1}: a_{i i}^{*}(z) a_{i-1, i-1}^{*}(w) a_{l, i-1}(z) a_{l, i-1}^{*}(w):\left\lfloor a_{j j}^{*} a_{i-1, i-1}\right\rfloor \\
&+\delta_{j, i-1}: a_{i i}^{*}(z) a_{i-1, i-1}^{*}(w):\left\lfloor a_{i-1, i-1} a_{i-1, i-1}^{*}\right\rfloor\left\lfloor a_{i-1, i-1}^{*} a_{i-1, j-1}\right\rfloor \\
&\left.+\delta_{j, i-1}: a_{i i}^{*}(z) a_{i-1, i-1}^{*}(w):\left(\sum_{l=1}^{i-1}\left\lfloor a_{l, i-1} a_{l, j-1}^{*}\right\rfloor a_{l, i-1}^{*} a_{l, j-1}\right\rfloor\right) .
\end{aligned}
$$

e assim,

$$
\begin{aligned}
& \begin{aligned}
(f)\left[\sum_{k=1}^{i-1}:\right. & \left.a_{i i}^{*} a_{k, i-1} a_{k, i-1}^{*}:, \sum_{l=1}^{j}: a_{j j}^{*} a_{l, j} a_{l, j}^{*}:\right] \\
= & \delta_{j, i-1} \sum_{k=1}^{i-1}: a_{i i}^{*}(z) a_{i-1, i-1}^{*}(z) a_{k, i-1}(w) a_{k, i-1}^{*}(w): \delta(z / w) \\
& +\delta_{i j} \sum_{l=1}^{i-1}: a_{i i}^{*}(w) a_{i i}^{*}(w) a_{l, i-1}(z) a_{l, i-1}^{*}(z): \delta(z / w) \\
& \quad-i \delta_{1 \leq i-1 \leq r} \delta_{j, i-1}: a_{i i}^{*}(z) a_{i-1, i-1}^{*}(w): \partial_{w} \delta(z / w) .
\end{aligned} \\
& (g)\left[\sum_{k=1}^{i}: a_{i i}^{*} a_{k i} a_{k i}^{*}:, \sum_{l=1}^{j}: a_{j j}^{*} a_{l j} a_{l j}^{*}:\right]=-(3+i) \delta_{1 \leq i \leq r} \delta_{i j}: a_{i i}^{*}(z) a_{i i}^{*}(w): \partial_{w} \delta(z / w) .
\end{aligned}
$$


Agora não temos contrações duplas para $i=j+1 \leq r, l=i$ e $k=j$. Logo

$$
\begin{aligned}
\text { (h) } \sum_{l=j+1}^{n}\left[\sum_{k=1}^{i-1}: a_{i i}^{*} a_{k, i-1} a_{k, i-1}^{*}:-\sum_{k=1}^{i}: a_{i i}^{*} a_{k i} a_{k i}^{*}:, a_{j+1, l}(w) a_{j l}^{*}(w)\right] \\
=-\delta_{j, i-1}\left(\sum_{k=1}^{i-1}: a_{i-1, i}^{*}(w) a_{k, i-1}(z) a_{k, i-1}^{*}(z):-\sum_{k=1}^{i}: a_{i-1, i}^{*}(w) a_{k i}(z) a_{k i}^{*}(z):\right) \delta(z / w) \\
\quad-\delta_{j+1 \leq i-1}: a_{i i}^{*} a_{j+1, i-1}(z) a_{j, i-1}^{*}(z): \delta(z / w)+\delta_{j+1 \leq i}: a_{i i}^{*} a_{j+1, i}(z) a_{j, i}^{*}(z): \delta(z / w) \\
\quad+\delta_{j+1 \leq i-1} a_{i i}^{*} a_{j+1, i-1}(z) a_{j, i-1}^{*}(z) \delta(z / w)-\delta_{j+1 \leq i}: a_{i i}^{*} a_{j+1, i}(z) a_{j, i}^{*}(z): \delta(z / w) \\
\quad+\delta_{i \leq r} \delta_{j, i-1} a_{i-1, i}^{*}(z) \partial_{w} \delta(z / w) \\
=-\delta_{j, i-1}\left(\sum_{k=1}^{i-1}: a_{i-1, i}^{*}(w) a_{k, i-1}(z) a_{k, i-1}^{*}(z):-\sum_{k=1}^{i}: a_{i-1, i}^{*}(w) a_{k i}(z) a_{k i}^{*}(z):\right) \delta(z / w) \\
\quad+\delta_{i \leqslant r} \delta_{j, i-1} a_{i-1, i}^{*}(z) \partial_{w} \delta(z / w) .
\end{aligned}
$$

Em (i), também não temos contrações duplas, o que nos dá

$$
\begin{aligned}
(i) \sum_{k=1}^{i-1}\left[: a_{i i}^{*} a_{k, i-1} a_{k, i-1}^{*}:, \sum_{l=1}^{j-1} a_{l, j-1} a_{l j}^{*}\right]-\sum_{k=1}^{i}\left[: a_{i i}^{*} a_{k i} a_{k i}^{*}:, \sum_{l=1}^{j-1} a_{l, j-1} a_{l j}^{*}\right] \\
=\left(-\delta_{i, j-1} \sum_{k=1}^{i-1}: a_{k, i-1} a_{k, i-1}^{*} a_{i, i+1}^{*}:-\delta_{i j} \sum_{k=1}^{i-1}: a_{i i}^{*} a_{k, i-1} a_{k, i}^{*}:\right. \\
\quad+\delta_{j, i-1} \sum_{k=1}^{i-2}: a_{i i}^{*} a_{k, i-2} a_{k, i-1}^{*}:+\delta_{i, j-1} \sum_{k=1}^{i}: a_{k i} a_{k i}^{*} a_{i, i+1}^{*}: \\
\left.\quad-\delta_{i j} \sum_{k=1}^{i-1}: a_{i i}^{*} a_{k, i-1} a_{k i}^{*}:+\delta_{i, j-1} \sum_{k=1}^{i}: a_{i i}^{*} a_{k i} a_{k, i+1}^{*}\right) \delta(z / w) \\
=\left(\delta_{j, i-1} \sum_{l=1}^{i-2}: a_{i i}^{*} a_{l, i-2} a_{l, i-1}^{*}:-2 \delta_{i j} \sum_{l=1}^{i-1}: a_{i i}^{*} a_{l, i-1} a_{l i}^{*}:+\delta_{i, j-1} \sum_{l=1}^{i}: a_{i i}^{*} a_{l i} a_{l, i+1}^{*}:\right. \\
\left.\quad-\delta_{i, j-1}\left(\sum_{l=1}^{i-1}: a_{i, i+1}^{*} a_{l, i-1} a_{l, i-1}^{*}:-\sum_{l=1}^{i}: a_{i, i+1}^{*} a_{l i} a_{l i}^{*}:\right)\right) \delta(z / w) .
\end{aligned}
$$

Para $(j)$ e para $(k)$, temos

$(j)\left[\sum_{k=1}^{i-1} a_{k, i-1} a_{k i}^{*}, \sum_{l=1}^{j-1} a_{l, j-1} a_{l j}^{*}\right]=\left(\delta_{j, i-1} \sum_{l=1}^{i-2}: a_{l, i-2} a_{l i}^{*}:-\delta_{i, j-1} \sum_{l=1}^{j-2}: a_{l, j-2} a_{l j}^{*}:\right) \delta(z / w)$

e também

$$
a_{i+1, k}(z) a_{i k}^{*}(z) a_{j+1, l}(w) a_{j l}^{*}(w)=a_{i+1, k}(z) a_{j l}^{*}(w)\left\lfloor a_{i k}^{*} a_{j+1, l}\right\rfloor+a_{i k}^{*}(z) a_{j+1, l}(w)\left\lfloor a_{i+1, k} a_{j l}^{*}\right\rfloor
$$

já que em (k), não temos contrações duplas (pois só temos $i+1=j, k=l$ e $i=j+1$ ). 
Assim

$$
\begin{aligned}
(k) & \sum_{k=i+1}^{n} \sum_{l=j+1}^{n}\left[a_{i+1, k}(z) a_{i k}^{*}(z), a_{j+1, l}(w) a_{j l}^{*}(w)\right] \\
= & \sum_{k=i+1}^{n} \sum_{l=j+1}^{n} a_{i+1, k}(z)\left[a_{i k}^{*}(z), a_{j+1, l}(w)\right] a_{j l}^{*}(w) \\
& +\sum_{k=i+1}^{n} \sum_{l=j+1}^{n} a_{i k}^{*}(z)\left[a_{i+1, k}(z), a_{j l}^{*}(w)\right] a_{j+1, l}(w) \\
= & \left(\delta_{i, j-1} \sum_{l=i+2}^{n}: a_{i+2, l} a_{i l}^{*}:-\delta_{j, i-1} \sum_{l=j+2}^{n}: a_{j+2, k} a_{j k}^{*}:\right) \delta(z / w) .
\end{aligned}
$$

Finalmente, temos

$$
\begin{aligned}
(l) & \sum_{k=1}^{i-1} \sum_{l=j+1}^{n}\left[a_{k, i-1}(z) a_{k i}^{*}(z), a_{j+1, l}(w) a_{j l}^{*}(w)\right] \\
& =\sum_{k=1}^{i-1} \sum_{l=j+1}^{n} a_{k i}^{*}(z) a_{j+1, l}(w) \delta_{k, j} \delta_{l, i-1} \delta(z / w)-\sum_{k=1}^{i-1} \sum_{l=j+1}^{n} a_{k, i-1}(z) a_{j l}^{*}(w) \delta_{k, j+1} \delta_{i, l} \delta(z / w) \\
& =\delta_{j \leq i-2} a_{j+1, i-1}(w) a_{j i}^{*}(z) \delta(z / w)-\delta_{j \leq i-2} a_{j+1, i-1}(z) a_{j i}^{*}(w) \delta(z / w) \\
& =0 .
\end{aligned}
$$

\subsubsection{A representação}

Teorema 3.1.27. Seja $\lambda \in \mathfrak{h}^{*}$ e seja $\lambda_{i}=\lambda\left(H_{i}\right)$. A função $\rho$ obtida através da função

$$
\begin{aligned}
& \rho\left(F_{i}\right)(z)=a_{i i}(z)+\sum_{j=i+1}^{n} a_{i j}(z) a_{i+1, j}^{*}(z), \\
& \rho\left(H_{i}\right)(z)=2: a_{i i}(z) a_{i i}^{*}(z):+\sum_{j=1}^{i-1}\left(: a_{j i}(z) a_{j i}^{*}(z):-: a_{j, i-1}(z) a_{j, i-1}^{*}(z):\right) \\
& +\sum_{j=i+1}^{n}\left(: a_{i j}(z) a_{i j}^{*}(z):-: a_{i+1, j}(z) a_{i+1, j}^{*}(z):\right)+b_{i}, \\
& \rho\left(E_{i}\right)(z)=\sum_{k=1}^{i-1}: a_{i i}^{*}(z) a_{k, i-1}(z) a_{k, i-1}^{*}(z):-\sum_{k=1}^{i}: a_{i i}^{*}(z) a_{k i}(z) a_{k i}^{*}(z): \\
& +\sum_{k=i+1}^{n} a_{i+1, k}(z) a_{i k}^{*}(z)-\sum_{k=1}^{i-1} a_{k, i-1}(z) a_{k i}^{*}(z)-a_{i i}^{*}(z) b_{i}(z) \\
& -\left(\delta_{i>r}(r+1)+\delta_{i \leqslant r}(i+1)-\gamma^{2}\right) \partial a_{i i}^{*}(z), \\
& \rho(c)=\gamma^{2}-(r+1) \text {, }
\end{aligned}
$$

escrita na linguagem de distribuições formais (ver Teorema 2.2.4) define uma ação no espaço de Fock $\mathbb{C}[\mathbf{x}] \otimes \mathbb{C}[\mathbf{y}]$ via geradores $c, E_{\text {im }}, F_{\text {im }}, H_{\text {im }}, i=1, \ldots, n$ e $m \in \mathbb{Z}$.

Para simplificar, denotemos $a_{i j}$ ao invés de $a_{i j}(z), a_{i j}^{*}$ ao invés de $a_{i j}^{*}(z)$ e $b_{i}$ ao invés 
de $b_{i}(z)$. Este teorema define uma realização tipo bóson de $\widehat{\mathfrak{s l}}(n+1, \mathbb{C})$ e uma estrutura de módulo no espaço de Fock $\mathbb{C}[\mathbf{x}] \otimes \mathbb{C}[\mathbf{y}]$ que depende do parâmetro $0 \leqslant r \leqslant n$ o qual chamamos de módulos intermediários de Wakimoto. Agora demonstremos o teorema acima checando as relações $\left(R_{1}\right)-\left(R_{6}\right)$.

Lema 3.1.28. $\left(R_{1}\right)\left[\rho\left(H_{i}\right)(z), \rho\left(H_{j}\right)(w)\right]=\left(\alpha_{i} \mid \alpha_{j}\right) \rho(c) \partial_{w} \delta(z / w)$.

Demonstração. Temos

$$
\begin{aligned}
{\left[\rho\left(H_{i}\right)(z), \rho\left(H_{j}\right)(w)\right]=\left[\mathcal{H}_{i}(z)+b_{i}(z), \mathcal{H}_{j}(z)+b_{j}(z)\right] } \\
=\left(-\left(\alpha_{i} \mid \alpha_{j}\right)\left(\left(1-\delta_{i>r} \delta_{j>r}\right)(r+1)+\frac{r}{2} \delta_{i, r+1} \delta_{j, r+1}\right)\right. \\
\left.\left.\quad+\left(\alpha_{i} \mid \alpha_{j}\right)\left(\gamma^{2}-\delta_{i>r} \delta_{j>r}(r+1)+\frac{r}{2} \delta_{i, r+1} \delta_{j, r+1}\right)\right)\right) \partial_{w} \delta(z / w) \\
=\left(\alpha_{i} \mid \alpha_{j}\right) \rho(c) \partial_{w} \delta(z / w) .
\end{aligned}
$$

Lema 3.1.29. $\left(R_{2}\right)\left[\rho\left(H_{i}\right)(z), \rho\left(E_{j}\right)(w)\right]=\left(\alpha_{i} \mid, \alpha_{j}\right) \rho\left(E_{j}\right)(z) \delta(z / w)$.

Demonstração. Temos

$$
\begin{aligned}
& {\left[\mathcal{H}_{i}(z), \rho\left(E_{j}\right)(w)\right]=\left[\mathcal{H}_{i}(z), \sum_{k=1}^{j-1}: a_{j j}^{*} a_{k, j-1} a_{k, j-1}^{*}:-\sum_{k=1}^{j}: a_{j j}^{*} a_{k j} a_{k j}^{*}\right):+\sum_{k=j+1}^{n} a_{j+1, k} a_{j k}^{*} } \\
& \left.-\sum_{k=1}^{j-1} a_{k, j-1} a_{k j}^{*}-a_{j j}^{*} b_{j}-\left(\delta_{j>r}(r+1)+\delta_{j \leq r}(j+1)-\gamma^{2}\right) \partial a_{j j}^{*}\right] \\
= & \sum_{k=1}^{j-1}\left[\mathcal{H}_{i}(z),: a_{j j}^{*} a_{k, j-1} a_{k, j-1}^{*}:\right]-\sum_{k=1}^{j}\left[\mathcal{H}_{i}(z), \quad: a_{j j}^{*} a_{k j} a_{k j}^{*}:\right] \\
& +\sum_{k=j+1}^{n}\left[\mathcal{H}_{i}(z), a_{j+1, k} a_{j k}^{*}\right]-\sum_{k=1}^{j-1}\left[\mathcal{H}_{i}(z), a_{k, j-1} a_{k j}^{*}\right]-\left[\mathcal{H}_{i}(z), a_{j j}^{*}\right] b_{j} \\
& -\left(\delta_{j>r}(r+1)+\delta_{j \leq r}(j+1)-\gamma^{2}\right)\left[\mathcal{H}_{i}(z), \partial_{w} a_{j j}^{*}(w)\right] \\
= & \sum_{k=1}^{j-1}\left(\left(\alpha_{i} \mid \alpha_{j}\right): a_{j j}^{*} a_{k, j-1} a_{k, j-1}^{*}: \delta(z / w)-\delta_{1 \leq j-1 \leq r}\left(\alpha_{i} \mid \alpha_{k}+\cdots+\alpha_{j-1}\right) a_{j j}^{*}(w) \partial_{w} \delta(z / w)\right) \\
& -\sum_{k=1}^{j}\left(\left(\alpha_{i} \mid \alpha_{j}\right): a_{j j}^{*} a_{k j} a_{k j}^{*}: \delta(z / w)\right. \\
& \left.-\delta_{1 \leq j \leq r}\left(\alpha_{i} \mid \alpha_{k}+\cdots+\alpha_{j}\right)\left(\delta_{j k} a_{k k}^{*}(w)+a_{j j}^{*}(w)\right) \partial_{w} \delta(z / w)\right) \\
& +\left(\alpha_{i} \mid \alpha_{j}\right) \sum_{k=j+1}^{n} a_{j+1, k}(z) a_{j k}^{*}(w) \delta(z / w)-\left(\alpha_{i} \mid \alpha_{j}\right) \sum_{k=1}^{j-1} a_{k, j-1}(z) a_{k j}^{*}(w) \delta(z / w) \\
& -\left(\alpha_{i} \mid \alpha_{j}\right) a_{j j}^{*}(z) b_{j}(w) \delta(z / w)-\left(\alpha_{i} \mid \alpha_{j}\right)\left(\delta_{j>r}(r+1)+\delta_{j \leq r}(j+1)-\gamma^{2}\right) a_{j j}^{*}(z) \partial_{w} \delta(z / w) \\
&
\end{aligned}
$$




$$
\begin{aligned}
= & \left(\alpha_{i} \mid \alpha_{j}\right)\left(\sum_{k=1}^{j-1}: a_{j j}^{*} a_{k, j-1} a_{k, j-1}^{*}:-\sum_{k=1}^{j}: a_{j j}^{*} a_{k j} a_{k j}^{*}:\right. \\
& \left.+\sum_{k=j+1}^{n} a_{j+1, k} a_{j k}^{*}-\sum_{k=1}^{j-1} a_{k, j-1} a_{k j}^{*}-a_{j j}^{*} b_{j}\right) \delta(z / w) \\
& +\sum_{k=1}^{j} \delta_{1 \leq j \leq r}\left(\alpha_{i} \mid \alpha_{k}+\cdots+\alpha_{j}\right)\left(\delta_{j k} a_{k k}^{*}(w)+a_{j j}^{*}(w)\right) \partial_{w} \delta(z / w) \\
& -\sum_{k=1}^{j-1}\left(\delta_{1 \leq j-1 \leq r}\left(\alpha_{i} \mid \alpha_{k}+\cdots+\alpha_{j-1}\right) a_{j j}^{*}(w) \partial_{w} \delta(z / w)\right) \\
& -\left(\alpha_{i} \mid \alpha_{j}\right)\left(\delta_{j>r}(r+1)+\delta_{j \leq r}(j+1)-\gamma^{2}\right) a_{j j}^{*}(z) \partial_{w} \delta(z / w) .
\end{aligned}
$$

e também

$$
\left[b_{i}(z), \quad \rho\left(E_{j}\right)(w)\right]=-\mathfrak{B}_{i j} a_{j j}(w) \partial_{w} \delta(z / w) .
$$

São três casos a considerar

- Caso $j \leq r$.

$$
\begin{aligned}
\sum_{k=1}^{j}\left(\alpha_{i} \mid \alpha_{k}+\cdots+\alpha_{j}\right)\left(\delta_{j k} a_{k k}^{*}(w)+a_{j j}^{*}(w)\right) \partial_{w} \delta(z / w) \\
\quad-\sum_{k=1}^{j-1}\left(\alpha_{i} \mid \alpha_{k}+\cdots+\alpha_{j-1}\right) a_{j j}^{*}(w) \partial_{w} \delta(z / w) \\
\quad-\left(\alpha_{i} \mid \alpha_{j}\right)\left(\delta_{j>r}(r+1)+\delta_{j \leq r}(j+1)-\gamma^{2}\right) a_{j j}^{*}(z) \partial_{w} \delta(z / w) \\
\quad-\left(\alpha_{i} \mid \alpha_{j}\right)\left(\gamma^{2}-\delta_{i>r} \delta_{j>r}(r+1)+\frac{r}{2} \delta_{i, r+1} \delta_{j, r+1}\right) a_{j j}(w) \partial_{w} \delta(z / w) \\
=(j+1)\left(\alpha_{i} \mid \alpha_{j}\right) a_{j j}^{*}(w) \partial_{w} \delta(z / w)-\left(\alpha_{i} \mid \alpha_{j}\right)\left(j+1-\gamma^{2}\right) a_{j j}^{*}(z) \partial_{w} \delta(z / w) \\
\quad-\left(\alpha_{i} \mid \alpha_{j}\right) \gamma^{2} a_{j j}(w) \partial_{w} \delta(z / w) \\
=-\left(\alpha_{i} \mid \alpha_{j}\right)\left(j+1-\gamma^{2}\right) \partial_{w} a_{j j}(w) \delta(z / w) .
\end{aligned}
$$

- Caso $j=r+1$.

$$
\begin{aligned}
-\sum_{k=1}^{r}( & \left.\left(\alpha_{i} \mid \alpha_{k}+\cdots+\alpha_{r}\right) a_{r+1, r+1}^{*}(w) \partial_{w} \delta(z / w)\right) \\
& -\left(\alpha_{i} \mid \alpha_{r+1}\right)\left((r+1)-\gamma^{2}\right) a_{r+1, r+1}^{*}(z) \partial_{w} \delta(z / w) \\
& -\left(\alpha_{i} \mid \alpha_{r+1}\right)\left(\gamma^{2}-\delta_{i>r}(r+1)+\frac{r}{2} \delta_{i, r+1}\right) a_{r+1, r+1}(w) \partial_{w} \delta(z / w) \\
= & -\left(\alpha_{i} \mid \alpha_{r+1}\right)\left((r+1)-\gamma^{2}\right)\left(\partial_{w} a_{r+1, r+1}^{*}(w)\right) \delta(z / w),
\end{aligned}
$$

que segue de 


$$
\begin{aligned}
& -\sum_{k=1}^{r}\left(\alpha_{i} \mid \alpha_{k}+\cdots+\alpha_{r}\right)+\left(\alpha_{i} \mid \alpha_{r+1}\right)\left(\delta_{i>r}(r+1)-\frac{r}{2} \delta_{i, r+1}\right) \\
& \quad= \begin{cases}-2 & \text { se } 1=i=r \\
0 & \text { se } 1 \leq i<r \\
-(r+1) & \text { se } 1<i=r \\
2(r+1) & \text { se } 1 \leq i=r+1 \\
\left(\alpha_{i} \mid \alpha_{r+1}\right)(r+1) & \text { se } i>r+1\end{cases} \\
& =\left(\alpha_{i} \mid \alpha_{r+1}\right)(r+1)
\end{aligned}
$$

já que $\left(\alpha_{i} \mid \alpha_{j}\right)=0$ para $i \leq r<r+1<j$. Juntando todos estes casos, temos

$$
\left[\rho\left(H_{i}\right)(z), \rho\left(E_{j}\right)(w)\right]=\left(\alpha_{i} \mid \alpha_{j}\right) \rho\left(E_{j}\right)(w) \delta(z / w) .
$$

provando $\left(R_{2}\right)$.

Lema 3.1.30. $\left(R_{3}\right)\left[\rho\left(H_{i}\right)(z), \rho\left(F_{j}\right)(w)\right]=-\left(\alpha_{i} \mid \alpha_{j}\right) \rho\left(F_{j}\right)(z) \delta(z / w)$.

Demonstração. Temos

$$
\begin{aligned}
& {\left[\rho\left(H_{i}\right)(z), \rho\left(F_{j}\right)(w)\right]=\left[\mathcal{H}_{i}(z), a_{j j}(w)+\sum_{k=j+1}^{n} a_{j k}(w) a_{j+1, k}^{*}(w)\right] } \\
&=-\left(\alpha_{i} \mid \alpha_{j}\right) a_{j j}(w) \delta(z / w)+\sum_{k=j+1}^{n}\left[\mathcal{H}_{i}(z), a_{j k}(w)\right] a_{j+1, k}^{*}(w) \\
&+\sum_{k=j+1}^{n} a_{j k}(w)\left[\mathcal{H}_{i}(z), a_{j+1, k}^{*}(w)\right] \\
&=\left(-\left(\alpha_{i} \mid \alpha_{j}\right) a_{j j}(w)-\sum_{k=j+1}^{n}\left(\alpha_{i} \mid \alpha_{j}+\cdots+\alpha_{k}\right) a_{j k}(z) a_{j+1, k}^{*}(w)\right. \\
&\left.+\sum_{k=j+1}^{n}\left(\alpha_{i} \mid \alpha_{j+1}+\cdots+\alpha_{k}\right) a_{j k}(w) a_{j+1, k}^{*}(z)\right) \delta(z / w) \\
&=-\left(\alpha_{i} \mid \alpha_{j}\right) \rho\left(F_{j}\right)(z) \delta(z / w) .
\end{aligned}
$$

Lema 3.1.31. $\left(R_{4}\right)\left[\rho\left(E_{i}\right)(z), \rho\left(F_{j}\right)(w)\right]=\delta_{i j}\left(\rho\left(H_{i}\right)(z) \delta(z / w)+\rho(c) \partial_{w} \delta(z / w)\right)$ 
Demonstração. Temos

$$
\begin{aligned}
& {\left[\rho\left(E_{i}\right)(z), \rho\left(F_{j}\right)(w)\right]=\delta_{i j}\left(\left[\rho\left(E_{i}\right)(z), a_{i i}(w)\right]+\sum_{l=i+1}^{n}\left[\rho\left(E_{i}\right)(z), a_{i l}(w) a_{i+1, l}^{*}(w)\right]\right)} \\
& \quad+\delta_{i \neq j}\left(\left[\rho\left(E_{i}\right)(z), a_{j j}(w)\right]+\sum_{l=j+1}^{n}\left[\rho\left(E_{i}\right)(z), a_{j l}(w) a_{j+1, l}^{*}(w)\right]\right) \\
& =\delta_{i j}\left(\left(2: a_{i i}(z) a_{i i}^{*}(z):-\sum_{k=1}^{i-1}\left(: a_{k, i-1} a_{k, i-1}^{*}:-: a_{k i} a_{k i}^{*}:\right)+b_{i}(z)\right) \delta(z / w)\right. \\
& \left.\quad+\left(\delta_{i>r}(r+1)+\delta_{i=<r}(i+1)-\gamma^{2}\right) \partial_{z} \delta(z / w)\right) \\
& \quad+\delta_{i j}\left(\sum_{l=i+1}^{n}\left[\sum_{k=i+1}^{n} a_{i+1, k}(z) a_{i k}^{*}(z), a_{i l}(w) a_{i+1, l}^{*}(w)\right]\right) \\
& \quad+\delta_{i \neq j}\left(-\delta_{j, i-1} a_{i-1, i-1}(z) a_{i i}^{*}(z) \delta(z / w)\right)+\delta_{i \neq j}\left(\delta_{j, i-1} a_{i-1, i-1}(z) a_{i i}^{*}(z) \delta(z / w)\right) \\
& =\delta_{i j}\left(\rho\left(H_{i}\right)(z) \delta(z / w)+\rho(c) \partial_{w} \delta(z / w)\right) .
\end{aligned}
$$

Finalmente, só nos falta provar as relações de Serre.

Lema 3.1.32. $\left(R_{5} \mid R_{6}\right)$

$$
\begin{aligned}
{\left[\rho\left(F_{i}\right)(z), \rho\left(F_{j}\right)(w)\right] } & =\left[\rho\left(E_{i}\right)(z), \rho\left(E_{j}\right)(w)\right]=0 \text { se }\left(\alpha_{i} \mid \alpha_{j}\right) \neq-1 \\
{\left[\rho\left(F_{i}\right)\left(z_{1}\right), \rho\left(F_{i}\right)\left(z_{2}\right), \rho\left(F_{j}\right)(w)\right] } & =\left[\rho\left(E_{i}\right)\left(z_{1}\right), \rho\left(E_{i}\right)\left(z_{2}\right), \rho\left(E_{j}\right)(w)\right]=0, \\
& \text { se }\left(\alpha_{i} \mid \alpha_{j}\right)=-1 .
\end{aligned}
$$

Demonstração. Temos

$$
\begin{aligned}
{\left[\rho\left(F_{i}\right)(z), \rho\left(F_{j}\right)(w)\right]=\left[a_{i i}(z), a_{j j}(w)+\sum_{l=j+1}^{n} a_{j l}(w) a_{j+1, l}^{*}(w)\right] } \\
+\left[\sum_{k=i+1}^{n} a_{i k}(z) a_{i+1, k}^{*}(z), a_{j j}(w)+\sum_{l=j+1}^{n} a_{j l}(w) a_{j+1, l}^{*}(w)\right] \\
=\delta_{i, j+1} a_{j, j+1}(w) \delta(z / w)+\left(\delta_{i, j+1} \sum_{k=j+2}^{n} a_{j k}(z) a_{j+2, k}^{*}(z)\right. \\
\\
\left.-\delta_{j, i+1} \sum_{k=i+2}^{n} a_{i k}(z) a_{i+2, k}^{*}(z)-\delta_{j, i+1} a_{i, i+1}(z)\right) \delta(z / w) \\
=\left(\delta_{i, j+1} a_{i-1, i}-\delta_{j, i+1} a_{i, i+1}\right) \delta(z / w) \\
+\left(\delta_{i, j+1} \sum_{k=i+1}^{n} a_{i-1, k} a_{i+1, k}^{*}-\delta_{j, i+1} \sum_{k=i+2}^{n} a_{i k} a_{i+2, k}^{*}\right) \delta(z / w) .
\end{aligned}
$$


Como todas as parcelas aparecem multiplicadas por $\delta_{j, i+1}$ ou $\delta_{i, j+1}$, temos que

$$
\left[\rho\left(F_{i}\right)(z), \rho\left(F_{j}\right)(w)\right]=0
$$

quando $|i-j| \neq 1$, isto é, quando $\left(\alpha_{i}, \alpha_{j}\right) \neq-1$. Agora para $j=i+1$, temos

$$
\begin{aligned}
{\left[\rho\left(F_{i}\right)\left(z_{1}\right), \rho\left(F_{i}\right)\left(z_{1}\right), \rho\left(F_{i+1}\right)(w)\right]=\left[\rho\left(F_{i}\right)\left(z_{1}\right),\left[\rho\left(F_{i}\right)\left(z_{1}\right), \rho\left(F_{i+1}\right)(w)\right]\right] } \\
\quad=\left[\rho\left(F_{i}\right)\left(z_{1}\right),-a_{i, i+1}\left(z_{2}\right)-\sum_{k=i+2}^{n} a_{i k} a_{i+2, k}^{*}\right] \delta(z / w) \\
\quad=\left[a_{i i}\left(z_{1}\right)+\sum_{l=i+1}^{n} a_{i l}\left(z_{1}\right) a_{i+1, l}^{*}\left(z_{1}\right),-a_{i, i+1}(z)-\sum_{k=i+2}^{n} a_{i k} a_{i+2, k}^{*}\right] \delta(z / w) \\
\quad=0 .
\end{aligned}
$$

Analogamente, fazemos os cálculos para $i=j+1$, provando $\left(R_{5} \mid R_{6}\right)$ para $\rho\left(F_{i}\right)(z)$. Só nos resta prová-lo para $\rho\left(E_{i}\right)(z)$. Defina

$$
\begin{aligned}
& \rho\left(E_{i}^{1}\right)(z)=\sum_{k=1}^{i-1}: a_{i i}^{*} a_{k, i-1} a_{k, i-1}^{*}:-\sum_{k=1}^{i}: a_{i i}^{*} a_{k i} a_{k i}^{*}:, \\
& \rho\left(E_{i}^{2}\right)(z)=\sum_{k=i+1}^{n} a_{i+1, k} a_{i k}^{*}-\sum_{k=1}^{i-1} a_{k, i-1} a_{k i}^{*}, \\
& \rho\left(E_{i}^{3}\right)(z)=-a_{i i}^{*} b_{i}-\left(\delta_{i>r}(r+1)+\delta_{i \leq r}(i+1)-\gamma^{2}\right) \partial a_{i i}^{*} .
\end{aligned}
$$

Temos os seguintes colchetes:

$$
\begin{aligned}
& (a)\left[\rho\left(E_{i}^{1}\right)(z), \rho\left(E_{j}^{1}\right)(w)\right]=\delta_{j, i-1} \sum_{k=1}^{i-2}: a_{i i}^{*}(z) a_{i-1, i-1}^{*}(z) a_{k, i-2}(w) a_{k, i-2}^{*}(w): \delta(z / w) \\
& \quad-\delta_{i, j-1} \sum_{l=1}^{j-2}: a_{j j}^{*}(w) a_{j-1, j-1}^{*}(w) a_{l, j-2}(z) a_{l, j-2}^{*}(z): \delta(z / w) \\
& \quad-\delta_{j, i-1} \sum_{k=1}^{i-1}: a_{i i}^{*}(z) a_{i-1, i-1}^{*}(z) a_{k, i-1}(w) a_{k, i-1}^{*}(w): \delta(z / w) \\
& \quad+\delta_{i, j-1} \sum_{k=1}^{j-1}: a_{j j}^{*}(z) a_{j-1, j-1}^{*}(z) a_{k, j-1}(w) a_{k, j-1}^{*}(w): \delta(z / w) \\
& \quad-(i-1) \delta_{1 \leq i-1 \leq r} \delta_{i j}: a_{i i}^{*}(z) a_{i i}^{*}(w): \partial_{w} \delta(z / w) \\
& \quad+i \delta_{1 \leq i-1 \leq r} \delta_{j, i-1}: a_{i i}^{*}(z) a_{i-1, i-1}^{*}(w): \partial_{w} \delta(z / w) \\
& \quad-j \delta_{1 \leq j-1 \leq r} \delta_{i, j-1}: a_{j j}^{*}(w) a_{j-1, j-1}^{*}(z): \partial_{z} \delta(z / w) \\
& \quad-(3+i) \delta_{1 \leq i \leq r} \delta_{i j}: a_{i i}^{*}(z) a_{i i}^{*}(w): \partial_{w} \delta(z / w) .
\end{aligned}
$$




$$
\begin{aligned}
& (b)\left[\rho\left(E_{i}^{1}\right)(z), \rho\left(E_{j}^{2}\right)(w)\right]+\left[\rho\left(E_{i}^{2}\right)(z), \rho\left(E_{j}^{1}\right)(w)\right] \\
& =-\delta_{j, i-1}\left(\sum_{k=1}^{i-2}: a_{i-1, i}^{*} a_{k, i-2} a_{k, i-2}^{*}:-\sum_{k=1}^{i}: a_{i-1, i}^{*} a_{k i} a_{k i}^{*}:\right) \delta(z / w) \\
& +\delta_{i, j-1}\left(\sum_{k=1}^{i-1}: a_{i, i+1}^{*} a_{k, i-1} a_{k, i-1}^{*}:-\sum_{k=1}^{i+1}: a_{i, i+1}^{*} a_{k, i+1} a_{k, i+1}^{*}:\right) \delta(z / w) \\
& +\left(-\delta_{i-1, j} \sum_{l=1}^{i-2}: a_{i i}^{*} a_{l, i-2} a_{l, i-1}^{*}:+\delta_{i, j-1} \sum_{l=1}^{i}: a_{i i}^{*} a_{l, i} a_{l, i+1}^{*}:\right) \delta(z / w) \\
& +\left(\delta_{j-1, i} \sum_{l=1}^{j-2}: a_{j j}^{*} a_{l, j-2} a_{l, j-1}^{*}:+\delta_{j, i-1} \sum_{l=1}^{j}: a_{j j}^{*} a_{l, j} a_{l, j+1}^{*}:\right) \delta(z / w) \\
& +\delta_{i \leq r} \delta_{j, i-1} a_{i-1, i}^{*}(z) \partial_{w} \delta(z / w)-\delta_{j \leq r} \delta_{i, j-1} a_{j-1, j}^{*}(w) \partial_{z} \delta(z / w) \\
& (c)\left[\rho\left(E_{i}^{1}\right)(z) \rho\left(E_{j}^{3}\right)(w)\right]+\left[\rho\left(E_{i}^{3}\right)(z), \rho\left(E_{j}^{1}\right)(w)\right] \\
& =\left(-\delta_{i-1, j} a_{i i}^{*} a_{i-1, i-1}^{*} b_{j}+\delta_{j-1, i} a_{j j}^{*} a_{j-1, j-1}^{*} b_{i}\right) \delta(z / w) \\
& +\left(\delta_{j>r}(r+1)+\delta_{j \leq r}(j+1)-\gamma^{2}\right)\left(-\delta_{i-1, j} a_{i i}^{*}(z) a_{i-1, i-1}^{*}(z)+\delta_{i j} a_{i i}^{*}(z) a_{i i}^{*}(z)\right) \partial_{w} \delta(z / w) \\
& +\left(\delta_{i>r}(r+1)+\delta_{i \leq r}(i+1)-\gamma^{2}\right)\left(\delta_{j-1, i} a_{j j}^{*}(w) a_{j-1, j-1}^{*}(w)-\delta_{i j} a_{j j}^{*}(w) a_{j j}^{*}(w)\right) \partial_{z} \delta(z / w) . \\
& (d)\left[\rho\left(E_{i}^{2}\right)(z), \rho\left(E_{j}^{2}\right)(w)\right]=\left(\delta_{j, i-1} \sum_{l=1}^{i-2}: a_{l, i-2} a_{l i}^{*}:-\delta_{i, j-1} \sum_{l=1}^{j-2}: a_{l, j-2} a_{l j}^{*}:\right) \delta(z / w) \\
& +\left(\delta_{i, j-1} \sum_{l=i+2}^{n}: a_{i+2, l} a_{i l}^{*}:-\delta_{j, i-1} \sum_{l=j+2}^{n}: a_{j+2, k} a_{j k}^{*}:\right) \delta(z / w) \text {. } \\
& (e)\left[\rho\left(E_{i}^{2}\right)(z), \rho\left(E_{j}^{3}\right)(w)\right]+\left[\rho\left(E_{i}^{3}\right)(z), \rho\left(E_{j}^{2}\right)(w)\right] \\
& =\left(-\delta_{j, i+1} a_{i, i+1}^{*} b_{i+1}+\delta_{j, i-1} a_{i-1, i}^{*} b_{i-1}\right) \delta(z / w) \\
& -\left(\delta_{j>r}(r+1)+\delta_{j \leq r}(j+1)-\gamma^{2}\right)\left(\delta_{j, i+1} a_{i, i+1}^{*}(z)-\delta_{j, i-1} a_{i-1, i}^{*}(z)\right) \partial_{w} \delta(z / w) \\
& -\left(-\delta_{i, j+1} a_{j, j+1}^{*} b_{j+1}+\delta_{i, j-1} a_{j-1, j}^{*} b_{j-1}\right) \delta(z / w) \\
& +\left(\delta_{i>r}(r+1)+\delta_{i \leq r}(i+1)-\gamma^{2}\right)\left(\delta_{i, j+1} a_{j, j+1}^{*}(w)-\delta_{i, j-1} a_{j-1, j}^{*}(w)\right) \partial_{z} \delta(z / w) . \\
& (f)\left[\rho\left(E_{i}^{3}\right)(z), \rho\left(E_{j}^{3}\right)(w)\right]=\left[-a_{i i}^{*} b_{i}-\left(\delta_{i>r}(r+1)+\delta_{i \leq r}(i+1)-\gamma^{2}\right) \partial a_{i i}^{*}\right. \text {, } \\
& \left.-a_{j j}^{*} b_{j}-\left(\delta_{j>r}(r+1)+\delta_{j \leq r}(j+1)-\gamma^{2}\right) \partial a_{j j}^{*}\right]=a_{i i}^{*}(z) a_{j j}^{*}(w) \mathfrak{B}_{i j} \partial_{w} \delta(z / w) .
\end{aligned}
$$

Agora todas as parcelas acima são nulas se $|i-j| \notin\{0,1\}$, isto é, se $\left(\alpha_{i} \mid \alpha_{j}\right)=0$ já que todos os termos estão multiplicados por $\delta_{i j}, \delta_{j, i+1}$ ou $\delta_{i, j+1}$ e, então,

$$
\left[\rho\left(E_{i}\right)(z), \rho\left(E_{j}\right)(w)\right]=0, \quad \text { se } \quad\left(\alpha_{i} \mid \alpha_{j}\right)=0 .
$$


Ainda nos resta mostrar o que acontece quando $i=j$. Temos

$$
\begin{aligned}
{\left[\rho\left(E_{i}\right)(z), \rho(\right.} & \left.\left.E_{i}\right)(w)\right]=-(i-1) \delta_{1 \leq i-1 \leq r}: a_{i i}^{*}(z) a_{i i}^{*}(w): \partial_{w} \delta(z / w) \\
& -(3+i) \delta_{1 \leq i \leq r}: a_{i i}^{*}(z) a_{i i}^{*}(w): \partial_{w} \delta(z / w) \\
& +\left(\delta_{i>r}(r+1)+\delta_{i \leq r}(i+1)-\gamma^{2}\right) a_{i i}^{*}(z) a_{i i}^{*}(z) \partial_{w} \delta(z / w) \\
& -\left(\delta_{i>r}(r+1)+\delta_{i \leq r}(i+1)-\gamma^{2}\right) a_{i i}^{*}(w) a_{i i}^{*}(w) \partial_{z} \delta(z / w) \\
& +2 a_{i i}^{*}(z) a_{i i}^{*}(w)\left(\gamma^{2}+\left(\delta_{1 \leq i \leq r}-1\right)(r+1)+\delta_{i, r+1} \frac{r}{2}\right) \partial_{w} \delta(z / w) \\
= & -\left((i-1) \delta_{1 \leq i-1 \leq r}+(3+i) \delta_{1 \leq i \leq r}-\delta_{i, r+1} r\right): a_{i i}^{*}(z) a_{i i}^{*}(w): \partial_{w} \delta(z / w) \\
& +\delta_{i \leq r}(i+1)\left(a_{i i}^{*}(z) a_{i i}^{*}(z)+a_{i i}^{*}(w) a_{i i}^{*}(w)\right) \partial_{z} \delta(z / w) \\
& +(r+1) \delta_{i>r}\left(a_{i i}^{*}(z) a_{i i}^{*}(z)+a_{i i}^{*}(w) a_{i i}^{*}(w)-2 a_{i i}^{*}(z) a_{j j}^{*}(w)\right) \partial_{w} \delta(z / w) \\
& +\gamma^{2}\left(2 a_{i i}^{*}(z) a_{i i}^{*}(w)-a_{i i}^{*}(z) a_{i i}^{*}(z)-a_{i i}^{*}(w) a_{i i}^{*}(w)\right) \partial_{w} \delta(z / w) \\
= & 0 .
\end{aligned}
$$

E, assim, mostramos que

$$
\left[\rho\left(E_{i}\right)(z), \quad \rho\left(E_{j}\right)(w)\right]=0 \quad \text { se } \quad\left(\alpha_{i} \mid \alpha_{j}\right) \neq-1 .
$$

Agora mostremos que $\left[\rho\left(E_{i}\right)\left(z_{1}\right), \rho\left(E_{i}\right)\left(z_{2}\right), \rho\left(E_{j}\right)(w)\right]=0$, se $\left(\alpha_{i} \mid \alpha_{j}\right)=-1$.

$$
\begin{aligned}
{\left[\rho\left(E_{i}\right)(z), \rho(\right.} & \left.\left.E_{i-1}\right)(w)\right]=\left(\sum_{k=1}^{i-2}: a_{i i}^{*}(z) a_{i-1, i-1}^{*}(z) a_{k, i-2}(w) a_{k, i-2}^{*}(w):\right. \\
- & \left.\sum_{k=1}^{i-1}: a_{i i}^{*}(z) a_{i-1, i-1}^{*}(z) a_{k, i-1}(w) a_{k, i-1}^{*}(w):\right) \delta(z / w) \\
& +\left(-\sum_{k=1}^{i-2}: a_{i-1, i}^{*} a_{k, i-2} a_{k, i-2}^{*}:-\sum_{k=1}^{i}: a_{i-1, i}^{*} a_{k i} a_{k i}^{*}:\right) \delta(z / w) \\
& -\sum_{l=1}^{i-2}: a_{i i}^{*} a_{l, i-2} a_{l, i-1}^{*}: \delta(z / w)+\sum_{l=1}^{i-1}: a_{i-1, i-1}^{*} a_{l, i-1} a_{l i}^{*}: \delta(z / w) \\
& +\sum_{l=1}^{i-2}: a_{l, i-2} a_{l i}^{*}: \delta(z / w)-\sum_{l=i+1}^{n}: a_{i+1, k} a_{i-1, k}^{*}: \delta(z / w) \\
& -a_{i i}^{*} a_{i-1, i-1}^{*} b_{i-1} \delta(z / w)+a_{i-1, i}^{*}\left(b_{i-1}+b_{i}\right) \delta(z / w) \\
& +\left(\delta_{i>r}(r+1)+\delta_{i \leq r}(i+1)-\gamma^{2}\right) \partial_{w} a_{i-1, i}^{*}(w) \delta(z / w) \\
& +a_{i i}^{*}(z) \partial_{w} a_{i-1, i-1}^{*}(w)\left(\gamma^{2}-\delta_{i-1 \leq r} i-\delta_{i-1>r}(r+1)\right) \delta(z / w) .
\end{aligned}
$$

Portanto, temos que 


$$
\begin{aligned}
(g)[ & \left.\rho\left(E_{i}^{1}\right)\left(z_{1}\right), \quad \rho\left(E_{i}\right)\left(z_{2}\right), \rho\left(E_{i-1}\right)(w)\right] \\
= & \sum_{k=1}^{i-2}: a_{i i}^{*} a_{i-1, i} a_{k, i-2} a_{k, i-2}^{*}: \delta\left(z_{1} / w\right) \delta\left(z_{2} / w\right) \\
& +\left(\sum_{k=1}^{i}: a_{i i}^{*} a_{i-1, i}^{*} a_{k i} a_{k i}^{*}:-\sum_{k=1}^{i-1}: a_{i i}^{*} a_{i-1, i}^{*} a_{k, i-1} a_{k, i-1}^{*}:\right) \delta\left(z_{1} / w\right) \delta\left(z_{2} / w\right) \\
& -\sum_{k=1}^{i}: a_{i i}^{*} a_{i-1, i}^{*} a_{k i} a_{k i}^{*}: \delta\left(z_{1} / w\right) \delta\left(z_{2} / w\right) \\
& +i \delta_{1 \leq i-1 \leq r}: a_{i i}^{*}\left(z_{1}\right) a_{i i}^{*}\left(z_{2}\right) a_{i-1, i-1}^{*}\left(z_{2}\right): \delta\left(z_{2} / w\right) \partial_{z_{2}} \delta\left(z_{1} / z_{2}\right) \\
& +(i+2) \delta_{1 \leq i \leq r} a_{i i}^{*}\left(z_{1}\right) a_{i-1, i}^{*}\left(z_{2}\right) \delta\left(z_{2} / w\right) \partial_{z_{2}} \delta\left(z_{1} / z_{2}\right) \\
& +\delta_{1 \leq i \leq r} a_{i i}^{*}\left(z_{2}\right) a_{i-1, i}^{*}\left(z_{1}\right) \delta\left(z_{2} / w\right) \partial_{z_{2}} \delta\left(z_{1} / z_{2}\right) \\
& -\delta_{1 \leq i-1 \leq r} a_{i i}^{*}\left(z_{1}\right) a_{i-1, i}^{*}\left(z_{2}\right) \delta\left(z_{2} / w\right) \partial_{z_{2}} \delta\left(z_{1} / z_{2}\right) \\
& -\sum_{l=1}^{i-2}: a_{i i}^{*} a_{l, i-2} a_{l i}^{*}: \delta\left(z_{1} / w\right) \delta\left(z_{2} / w\right)-: a_{i i}^{*} a_{i-1, i}^{*}:\left(b_{i-1}+b_{i}\right) \delta\left(z_{1} / z_{2}\right) \delta\left(z_{2} / w\right) \\
& -\left(\delta_{i>r}(r+1)+\delta_{i \leq r}(i+1)-\gamma^{2}\right): a_{i i}^{*}\left(z_{1}\right) a_{i-1, i}^{*}\left(z_{1}\right): \delta\left(z_{2} / w\right) \partial_{w} \delta\left(z_{1} / w\right) \\
& -\left(\gamma^{2}-\delta_{i-1 \leq r} i-\delta_{i-1>r}(r+1)\right): a_{i i}^{*}\left(z_{1}\right) a_{i i}^{*}\left(z_{1}\right) \partial_{w} a_{i-1, i-1}^{*}(w): \delta\left(z_{1} / z_{2}\right) \delta\left(z_{2} / w\right) \\
+ & \left(\gamma^{2}-\delta_{i-1 \leq r} i-\delta_{i-1>r}(r+1)\right): a_{i i}^{*}\left(z_{1}\right) a_{i i}^{*}\left(z_{2}\right) a_{i-1, i-1}^{*}\left(z_{1}\right): \partial_{w} \delta\left(z_{1} / w\right) \delta\left(z_{2} / w\right)
\end{aligned}
$$

$(h)\left[\rho\left(E_{i}^{2}\right)\left(z_{1}\right), \quad \rho\left(E_{i}\right)\left(z_{2}\right), \quad \rho\left(E_{i-1}\right)(w)\right]=\left(-\sum_{k=1}^{i-2}: a_{i i}^{*}\left(z_{2}\right) a_{i-1, i}^{*}\left(z_{2}\right) a_{k, i-2}(w) a_{k, i-2}^{*}(w):\right.$

$$
\begin{gathered}
+\sum_{k=1}^{i-1}: a_{i i}^{*} a_{i-1, i}^{*} a_{k, i-1} a_{k, i-1}^{*}:+\sum_{k=1}^{i-1}: a_{i-1, i}^{*} a_{k, i-1} a_{k i}^{*}:+\sum_{l=1}^{i-2}: a_{i i}^{*} a_{l, i-2} a_{l, i}^{*}: \\
\left.\quad-\sum_{l=1}^{i-1}: a_{i-1, i}^{*} a_{l, i-1} a_{l i}^{*}:+a_{i i}^{*} a_{i-1, i}^{*} b_{i-1}\right) \delta\left(z_{1} / z_{2}\right) \delta\left(z_{2} / w\right) \\
-\left(\gamma^{2}-\delta_{i-1 \leq r} i-\delta_{i-1>r}(r+1)\right): a_{i i}^{*}\left(z_{2}\right) a_{i-1, i}^{*}\left(z_{1}\right): \partial_{w} \delta\left(z_{1} / w\right) \delta\left(z_{2} / w\right) .
\end{gathered}
$$

$$
\begin{aligned}
(i)\left[\rho\left(E_{i}^{3}\right)\left(z_{1}\right),\right. & \left.\rho\left(E_{i}\right)\left(z_{2}\right), \rho\left(E_{i-1}\right)(w)\right]=: a_{i-1, i}^{*} a_{i i}^{*}: b_{i} \delta\left(z_{1} / z_{2}\right) \delta\left(z_{2} / w\right) \\
& +\left(\delta_{i>r}(r+1)+\delta_{i \leq r}(i+1)-\gamma^{2}\right) a_{i i}^{*}\left(z_{2}\right) a_{i-1, i}^{*}\left(z_{2}\right) \partial_{z_{1}} \delta\left(z_{1} / z_{2}\right) \delta\left(z_{2} / w\right) \\
& +\left(-a_{i i}^{*}\left(z_{1}\right) a_{i-1, i}^{*}\left(z_{2}\right)\left(\gamma^{2}-\delta_{i>r}(r+1)-\delta_{i, r+1}\right)\right. \\
& \left.-a_{i i}^{*}\left(z_{1}\right) a_{i i}^{*}\left(z_{2}\right) a_{i-1, i-1}^{*}\left(z_{2}\right)\left(\gamma^{2}-\delta_{i-1>r}(r+1)\right)\right) \delta\left(z_{2} / w\right) \partial_{z_{2}} \delta\left(z_{1} / z_{2}\right) .
\end{aligned}
$$

Somando as 3 parcelas, temos 


$$
\begin{aligned}
& {\left[\rho\left(E_{i}\right)\left(z_{1}\right), \rho\left(E_{i}\right)\left(z_{2}\right), \rho\left(E_{i-1}\right)(w)\right]=\left(i \delta_{1 \leq i-1 \leq r}: a_{i i}^{*}\left(z_{1}\right) a_{i i}^{*}\left(z_{2}\right) a_{i-1, i-1}^{*}\left(z_{2}\right):\right.} \\
& -\left(\gamma^{2}-\delta_{i-1 \leq r} i-\delta_{i-1>r}(r+1)\right): a_{i i}^{*}\left(z_{1}\right) a_{i i}^{*}\left(z_{1}\right) \partial_{w} a_{i-1, i-1}^{*}(w): \\
& +\left(\gamma^{2}-\delta_{i-1 \leq r} i-\delta_{i-1>r}(r+1)\right): a_{i i}^{*}\left(z_{1}\right) a_{i i}^{*}\left(z_{2}\right) a_{i-1, i-1}^{*}\left(z_{1}\right): \\
& \left.-\left(\gamma^{2}-\delta_{i-1>r}(r+1)\right) a_{i i}^{*}\left(z_{1}\right) a_{i i}^{*}\left(z_{2}\right) a_{i-1, i-1}^{*}\left(z_{2}\right)\right) \delta\left(z_{2} / w\right) \partial_{w} \delta\left(z_{1} / w\right) \\
& +(i+2) \delta_{1 \leq i \leq r} a_{i i}^{*}\left(z_{1}\right) a_{i-1, i}^{*}\left(z_{2}\right) \delta\left(z_{2} / w\right) \partial_{z_{2}} \delta\left(z_{1} / z_{2}\right) \\
& -\delta_{1 \leq i-1 \leq r} a_{i i}^{*}\left(z_{1}\right) a_{i-1, i}^{*}\left(z_{2}\right) \delta\left(z_{2} / w\right) \partial_{z_{2}} \delta\left(z_{1} / z_{2}\right) \\
& +\delta_{1 \leq i \leq r} a_{i i}^{*}\left(z_{2}\right) a_{i-1, i}^{*}\left(z_{1}\right) \delta\left(z_{2} / w\right) \partial_{z_{2}} \delta\left(z_{1} / z_{2}\right) \\
& -\left(\delta_{i>r}(r+1)+\delta_{i \leq r}(i+1)-\gamma^{2}\right): a_{i i}^{*}\left(z_{1}\right) a_{i-1, i}^{*}\left(z_{1}\right): \delta\left(z_{2} / w\right) \partial_{w} \delta\left(z_{1} / w\right) \\
& -\left(\gamma^{2}-\delta_{i-1 \leq r} i-\delta_{i-1>r}(r+1)\right): a_{i i}^{*}\left(z_{2}\right) a_{i-1, i}^{*}\left(z_{1}\right): \partial_{w} \delta\left(z_{1} / w\right) \delta\left(z_{2} / w\right) \\
& -\left(\delta_{i>r}(r+1)+\delta_{i \leq r}(i+1)-\gamma^{2}\right) a_{i i}^{*}\left(z_{2}\right) a_{i-1, i}^{*}\left(z_{2}\right) \delta\left(z_{2} / w\right) \partial_{w} \delta\left(z_{1} / w\right) \\
& -a_{i i}^{*}\left(z_{1}\right) a_{i-1, i}^{*}\left(z_{2}\right)\left(\gamma^{2}-\delta_{i>r}(r+1)-\delta_{i, r+1}\right) \delta\left(z_{2} / w\right) \partial_{w} \delta\left(z_{1} / w\right) \\
& =\left(\left((i+2) \delta_{1 \leq i \leq r}-\delta_{1 \leq i-1 \leq r}-\gamma^{2}+\delta_{i>r}(r+1)+\delta_{i, r+1}\right) a_{i i}^{*}\left(z_{1}\right) a_{i-1, i}^{*}\left(z_{2}\right)\right. \\
& +\left(\delta_{1 \leq i \leq r}-\gamma^{2}+\delta_{i-1 \leq r} i+\delta_{i-1>r}(r+1)\right): a_{i i}^{*}\left(z_{2}\right) a_{i-1, i}^{*}\left(z_{1}\right): \\
& -\left(\delta_{i>r}(r+1)+\delta_{i \leq r}(i+1)-\gamma^{2}\right): a_{i i}^{*}\left(z_{1}\right) a_{i-1, i}^{*}\left(z_{1}\right): \\
& \left.-\left(\delta_{i>r}(r+1)+\delta_{i \leq r}(i+1)-\gamma^{2}\right) a_{i i}^{*}\left(z_{2}\right) a_{i-1, i}^{*}\left(z_{2}\right)\right) \delta\left(z_{2} / w\right) \partial_{w} \delta\left(z_{1} / w\right) \\
& =\left(-\left(\delta_{i>r}(r+1)+\delta_{i \leq r}(i+1)-\gamma^{2}\right): a_{i i}^{*}\left(z_{1}\right) a_{i-1, i}^{*}\left(z_{1}\right):\right. \\
& +\left(\delta_{i>r}(r+1)+\delta_{i \leq r}(i+1)-\gamma^{2}\right) a_{i i}^{*}\left(z_{1}\right) a_{i-1, i}^{*}\left(z_{2}\right) \\
& -\left(\delta_{i>r}(r+1)+\delta_{i \leq r}(i+1)-\gamma^{2}\right) a_{i i}^{*}\left(z_{2}\right) a_{i-1, i}^{*}\left(z_{2}\right) \\
& \left.+\left(\delta_{i>r}(r+1)+\delta_{i \leq r}(i+1)-\gamma^{2}\right): a_{i i}^{*}\left(z_{2}\right) a_{i-1, i}^{*}\left(z_{1}\right):\right) \delta\left(z_{2} / w\right) \partial_{w} \delta\left(z_{1} / w\right) \\
& =0 \text {. }
\end{aligned}
$$

Agora só nos resta analisar o caso em que $j=i+1$. Como já analisamos o caso em que $j=i-1$ podemos aproveitar estes cálculos lembrando que

$$
\left[\rho\left(E_{i}\right)\left(z_{1}\right), \rho\left(E_{i}\right)\left(z_{2}\right), \rho\left(E_{i+1}\right)(w)\right]=-\left[\rho\left(E_{i}\right)\left(z_{1}\right), \rho\left(E_{i+1}\right)(w), \quad \rho\left(E_{i}\right)\left(z_{2}\right)\right]
$$

e substituindo $i$ por $i-1$. Assim, temos 


$$
\begin{aligned}
& (j)\left[\rho\left(E_{i-1}^{1}\right)\left(z_{1}\right), \rho\left(E_{i}\right)\left(z_{2}\right), \rho\left(E_{i-1}\right)(w)\right] \\
& =-(i-2) \delta_{i-2 \leq r}: a_{i i}^{*}\left(z_{2}\right) a_{i-1, i-1}^{*}\left(z_{1}\right) a_{i-1, i-1}^{*}\left(z_{2}\right) \delta\left(z_{2} / w\right) \partial_{z_{2}} \delta\left(z_{1} / z_{2}\right) \\
& -(i+2) \delta_{i-1 \leq r}: a_{i i}\left(z_{2}\right) a_{i-1, i-1}\left(z_{1}\right) a_{i-1, i-1}\left(z_{2}\right): \delta\left(z_{2} / w\right) \partial_{z_{2}} \delta\left(z_{1} / z_{2}\right) \\
& +(i-2) \delta_{i-2 \leq r}: a_{i-1, i-1}^{*}\left(z_{1}\right) a_{i-1, i}^{*}\left(z_{2}\right): \delta\left(z_{2} / w\right) \partial_{z_{2}} \delta\left(z_{1} / z_{2}\right) \\
& +2 \delta_{i-1 \leq r}: a_{i-1, i-1}^{*}\left(z_{1}\right) a_{i-1, i}^{*}\left(z_{2}\right): \delta\left(z_{2} / w\right) \partial_{z_{2}} \delta\left(z_{1} / z_{2}\right) \\
& +2 \sum_{l=1}^{i-2}: a_{i-1, i-1}^{*} a_{i i}^{*} a_{l, i-2} a_{l, i-1}^{*}: \delta\left(z_{1} / z_{2}\right) \delta\left(z_{2} / w\right) \\
& -\sum_{k=1}^{i-2}: a_{i-1, i-1}^{*} a_{i-1, i}^{*} a_{k, i-2} a_{k, i-2}^{*}: \delta\left(z_{1} / z_{2}\right) \delta\left(z_{2} / w\right) \\
& +\sum_{k=1}^{i-1}: a_{i-1, i}^{*} a_{i-1, i-1}^{*} a_{k, i-1} a_{k, i-1}^{*}: \delta\left(z_{1} / z_{2}\right) \delta\left(z_{2} / w\right) \\
& -\sum_{l=1}^{i-2}: a_{i-1, i-1}^{*} a_{l, i-2} a_{l i}^{*}: \delta\left(z_{1} / z_{2}\right) \delta\left(z_{2} / w\right)+: a_{i-1, i-1}^{*} a_{i-1, i-1}^{*} a_{i i}^{*}: b_{i-1} \delta\left(z_{1} / z_{2}\right) \delta\left(z_{2} / w\right) \\
& -\left(\gamma^{2}-\delta_{i-1 \leq r} i-\delta_{i-1>r}(r+1)\right): a_{i-1, i-1}^{*}\left(z_{1}\right) a_{i-1, i-1}^{*}\left(z_{1}\right) a_{i i}^{*}\left(z_{2}\right): \delta\left(z_{2} / w\right) \partial_{w} \delta\left(z_{1} / w\right) . \\
& (k)\left[\rho\left(E_{i-1}^{2}\right)\left(z_{1}\right), \rho\left(E_{i}\right)\left(z_{2}\right), \rho\left(E_{i-1}\right)(w)\right]=\sum_{k=1}^{i-2}: a_{i-1, i}^{*} a_{i-1, i-1}^{*} a_{k, i-2} a_{k, i-2}^{*}: \delta\left(z_{1} / z_{2}\right) \delta\left(z_{2} / w\right) \\
& -2 \sum_{k=1}^{i-2}: a_{i i}^{*} a_{i-1, i-1}^{*} a_{k, i-2} a_{k, i-1}^{*}: \delta\left(z_{1} / z_{2}\right) \delta\left(z_{2} / w\right)-\sum_{k=1}^{i-1}: a_{i-1, i}^{*} a_{i-1, i-1}^{*} a_{k, i-1} a_{k, i-1}^{*}: \delta\left(z_{1} / z_{2}\right) \delta\left(z_{2} / w\right) \\
& +\sum_{l=1}^{i-2}: a_{i-1, i-1}^{*} a_{l, i-2} a_{l i}^{*}: \delta\left(z_{1} / z_{2}\right) \delta\left(z_{2} / w\right)-a_{i-1, i}^{*} a_{i-1, i-1}^{*} b_{i-1} \delta\left(z_{1} / z_{2}\right) \delta\left(z_{2} / w\right) \\
& +\left(\gamma^{2}-\delta_{i-1 \leq r} i-\delta_{i-1>r}(r+1)\right) a_{i-1, i}^{*}\left(z_{1}\right) \partial_{w} a_{i-1, i-1}^{*}(w) \delta\left(z_{1} / z_{2}\right) \delta\left(z_{2} / w\right) . \\
& (l)\left[\rho\left(E_{i-1}^{3}\right)\left(z_{1}\right), \rho\left(E_{i}\right)\left(z_{2}\right), \rho\left(E_{i-1}\right)(w)\right]=-: a_{i i}^{*} a_{i-1, i-1}^{*} a_{i-1, i-1}^{*}: b_{i-1} \delta\left(z_{1} / z_{2}\right) \delta\left(z_{2} / w\right) \\
& +\left(\delta_{i-1>r}(r+1)+\delta_{i-1 \leq r} i-\gamma^{2}\right): a_{i i}^{*}\left(z_{2}\right) a_{i-1, i-1}^{*}\left(z_{2}\right) a_{i-1, i-1}^{*}\left(z_{2}\right): \delta\left(z_{2} / w\right) \partial_{z_{1}} \delta\left(z_{1} / z_{2}\right) \\
& +: a_{i-1, i-1}^{*} a_{i-1, i}^{*}: b_{i-1} \delta\left(z_{1} / z_{2}\right) \delta\left(z_{2} / w\right) \\
& +\left(\delta_{i-1>r}(r+1)+\delta_{i-1 \leq r} i-\gamma^{2}\right): a_{i-1, i-1}^{*}\left(z_{2}\right) a_{i-1, i}^{*}\left(z_{2}\right): \delta\left(z_{2} / w\right) \partial_{z_{1}} \delta\left(z_{1} / z_{2}\right) \\
& -\left(\gamma^{2}-(r+1) \delta_{i>r+2}-\delta_{i, r+2}\right) a_{i-1, i-1}^{*}\left(z_{1}\right) a_{i-1, i}^{*}\left(z_{2}\right) \delta\left(z_{2} / w\right) \partial_{z_{2}} \delta\left(z_{1} / z_{2}\right) \\
& +2\left(\gamma^{2}-(r+1) \delta_{i>r+1}+\frac{r}{2} \delta_{i, r+2}\right) a_{i-1, i-1}^{*}\left(z_{1}\right) a_{i-1, i-1}^{*}\left(z_{2}\right) a_{i, i}^{*}\left(z_{2}\right) \delta\left(z_{2} / w\right) \partial_{z_{2}} \delta\left(z_{1} / z_{2}\right) \text {. }
\end{aligned}
$$

Somando as 3 parcelas, temos 


$$
\begin{aligned}
& {\left[\rho\left(E_{i-1}\right)\left(z_{1}\right), \rho\left(E_{i}\right)\left(z_{2}\right), \rho\left(E_{i-1}\right)(w)\right]} \\
& =-(i-2) \delta_{i-2 \leq r}: a_{i i}^{*}\left(z_{2}\right) a_{i-1, i-1}^{*}\left(z_{1}\right) a_{i-1, i-1}^{*}\left(z_{2}\right) \delta\left(z_{2} / w\right) \partial_{z_{2}} \delta\left(z_{1} / z_{2}\right) \\
& -(i+2) \delta_{i-1 \leq r}: a_{i i}\left(z_{2}\right) a_{i-1, i-1}\left(z_{1}\right) a_{i-1, i-1}\left(z_{2}\right): \delta\left(z_{2} / w\right) \partial_{z_{2}} \delta\left(z_{1} / z_{2}\right) \\
& +(i-2) \delta_{i-2 \leq r}: a_{i-1, i-1}^{*}\left(z_{1}\right) a_{i-1, i}^{*}\left(z_{2}\right): \delta\left(z_{2} / w\right) \partial_{z_{2}} \delta\left(z_{1} / z_{2}\right) \\
& +2 \delta_{i-1 \leq r}: a_{i-1, i-1}^{*}\left(z_{1}\right) a_{i-1, i}^{*}\left(z_{2}\right): \delta\left(z_{2} / w\right) \partial_{z_{2}} \delta\left(z_{1} / z_{2}\right) \\
& -\left(\gamma^{2}-\delta_{i-1 \leq r} i-\delta_{i-1>r}(r+1)\right): a_{i-1, i-1}^{*}\left(z_{1}\right) a_{i-1, i-1}^{*}\left(z_{1}\right) a_{i i}^{*}\left(z_{2}\right): \delta\left(z_{2} / w\right) \partial_{w} \delta\left(z_{1} / w\right) \\
& +\left(\gamma^{2}-\delta_{i-1 \leq r} i-\delta_{i-1>r}(r+1)\right) a_{i-1, i}^{*}\left(z_{1}\right) \partial_{w} a_{i-1, i-1}^{*}(w) \delta\left(z_{1} / z_{2}\right) \delta\left(z_{2} / w\right) \\
& +\left(\delta_{i-1>r}(r+1)+\delta_{i-1 \leq r} i-\gamma^{2}\right): a_{i i}^{*}\left(z_{2}\right) a_{i-1, i-1}^{*}\left(z_{2}\right) a_{i-1, i-1}^{*}\left(z_{2}\right): \delta\left(z_{2} / w\right) \partial_{z_{1}} \delta\left(z_{1} / z_{2}\right) \\
& +\left(\delta_{i-1>r}(r+1)+\delta_{i-1 \leq r} i-\gamma^{2}\right): a_{i-1, i-1}^{*}\left(z_{2}\right) a_{i-1, i}^{*}\left(z_{2}\right): \delta\left(z_{2} / w\right) \partial_{z_{1}} \delta\left(z_{1} / z_{2}\right) \\
& -\left(\gamma^{2}-(r+1) \delta_{i>r+2}-\delta_{i, r+2}\right) a_{i-1, i-1}^{*}\left(z_{1}\right) a_{i-1, i}^{*}\left(z_{2}\right) \delta\left(z_{2} / w\right) \partial_{z_{2}} \delta\left(z_{1} / z_{2}\right) \\
& +2\left(\gamma^{2}-(r+1) \delta_{i>r+1}+\frac{r}{2} \delta_{i, r+2}\right) a_{i-1, i-1}^{*}\left(z_{1}\right) a_{i-1, i-1}^{*}\left(z_{2}\right) a_{i, i}^{*}\left(z_{2}\right) \delta\left(z_{2} / w\right) \partial_{z_{2}} \delta\left(z_{1} / z_{2}\right) \\
& =-\left(\gamma^{2}-\delta_{i-1 \leq r} i-\delta_{i-1>r}(r+1)\right): a_{i-1, i-1}^{*}\left(z_{1}\right) a_{i-1, i-1}^{*}\left(z_{1}\right) a_{i i}^{*}\left(z_{2}\right): \delta\left(z_{2} / w\right) \partial_{w} \delta\left(z_{1} / w\right) \\
& +2\left(\gamma^{2}-(r+1) \delta_{i>r+1}-i \delta_{i \leq r+1}\right) a_{i-1, i-1}^{*}\left(z_{1}\right) a_{i-1, i-1}^{*}\left(z_{2}\right) a_{i, i}^{*}\left(z_{2}\right) \delta\left(z_{2} / w\right) \partial_{z_{2}} \delta\left(z_{1} / z_{2}\right) \\
& +\left(\delta_{i-1>r}(r+1)+\delta_{i-1 \leq r} i-\gamma^{2}\right): a_{i i}^{*}\left(z_{2}\right) a_{i-1, i-1}^{*}\left(z_{2}\right) a_{i-1, i-1}^{*}\left(z_{2}\right): \delta\left(z_{2} / w\right) \partial_{z_{1}} \delta\left(z_{1} / z_{2}\right) \\
& +\left(\delta_{i-1>r}(r+1)+\delta_{i-1 \leq r} i-\gamma^{2}\right): a_{i-1, i-1}^{*}\left(z_{2}\right) a_{i-1, i}^{*}\left(z_{2}\right): \delta\left(z_{2} / w\right) \partial_{z_{1}} \delta\left(z_{1} / z_{2}\right) \\
& -\left(\gamma^{2}-\delta_{i-1 \leq r} i-\delta_{i-1>r}(r+1)\right) a_{i-1, i-1}^{*}\left(z_{1}\right) a_{i-1, i}^{*}\left(z_{2}\right) \delta\left(z_{2} / w\right) \partial_{z_{2}} \delta\left(z_{1} / z_{2}\right) \\
& +\left(\gamma^{2}-\delta_{i-1 \leq r} i-\delta_{i-1>r}(r+1)\right) a_{i-1, i}^{*}\left(z_{1}\right) \partial_{w} a_{i-1, i-1}^{*}(w) \delta\left(z_{1} / z_{2}\right) \delta\left(z_{2} / w\right) \\
& =0 \text {, }
\end{aligned}
$$

o que prova o Lema.

\subsection{Construção dos módulos $\mathbb{J}$-intermediários de Wakimoto}

Nesta seção e nas próximas, demonstramos os resultados obtidos em [Mar12].

\subsubsection{As $\mathbb{J}$-realizações boson}

Fixemos um subconjunto $\mathbb{J} \subseteq \mathbb{N}^{*}$. Primeiramente precisamos, em toda esta seção e na próxima, substituir $\mathbb{C}[\mathbf{y}]$ de $[\mathrm{CF} 04]$ por

$$
\mathbb{C}[\mathbf{y}]_{r}=\mathbb{C}\left[y_{i, m} \mid i,-m \in \mathbb{N}^{*}, 0 \leq i \leq r \text { or } r+2 \leq i \leq n\right] .
$$

Por abuso de notação, também denotaremos $\mathbb{C}[\mathbf{y}]_{r}$ por $\mathbb{C}[\mathbf{y}]$. Definamos a ação de $a_{i j, m}$, $a_{i j, m}^{*}$ e $b_{i m}$ da seguinte forma: 
$a_{i j, m}=\left\{\begin{array}{lr}\frac{\partial}{\partial x_{i j, m}}, & \text { se } m \geq 0 \quad \text { e } j \leq r, \\ x_{i j, m}, & \text { caso contrário }\end{array}\right.$
$a_{i j, m}^{*}=\left\{\begin{array}{lr}x_{i j,-m}, & \text { se } m \leq 0 \text { e } j \leq r, \\ -\frac{\partial}{\partial x_{i j,-m}}, & \text { caso contrário }\end{array}\right.$

Se $0 \leqslant i \leqslant r$, defina

$b_{i m}= \begin{cases}\lambda_{i}, & \text { se } \quad m=0, \\ m e_{i} \frac{\partial}{\partial y_{m}}, & \text { se } \quad m>0, \\ e_{i} y_{-m}, & \text { se } \quad m<0,\end{cases}$

e, se $r+2 \leqslant i \leqslant n$, defina

$b_{i m}=\left\{\begin{array}{lll}\lambda_{i}, & \text { se } \quad m=0, \\ m e_{i} \frac{\partial}{\partial y_{m}}, & \text { se } \quad m \in \mathbb{J}, \\ e_{i} y_{m}, & \text { se } \quad m \in \mathbb{N}^{*} \backslash \mathbb{J}, \\ -m e_{i} \frac{\partial}{\partial y_{-m}}, & \text { se } \quad-m \in \mathbb{N}^{*} \backslash \mathbb{J}, \\ e_{i} y_{-m}, & \text { se } \quad-m \in \mathbb{J} .\end{array}\right.$

Temos que redefinir $b_{r+1, m}$ (note que nossa definição de $b_{i m}$ para $i>r+1$ é um pouco diferente das de [CF04] e [CF05] mas aqui os colchetes também serão iguais a $\mathfrak{B}_{i j} \partial_{w} \delta(z / w)$ se $\left.i, j \neq r+1\right)$. Usamos o fato de que em $H=\left\langle H_{1}, \ldots, H_{n}\right\rangle$ temos $\left\langle H_{1}, \ldots, H_{r}\right\rangle \perp\left\langle H_{r+2}, \ldots, H_{n}\right\rangle$. Logo basta substituir $H_{r+1}$ por um conveniente $H_{r+1}^{*}$ tal que

$$
H_{r+1}^{*} \perp\left\langle H_{1}, \ldots, H_{r}, H_{r+2}, \ldots, H_{n}\right\rangle .
$$

Definição 3.2.2. Seja $H_{r+1}^{*}=\left(\sum_{i=1}^{r} \frac{i}{r+1} H_{i}\right)+H_{r+1}+\left(\sum_{j=1}^{n-r-1} \frac{j}{n-r} H_{n-j+1}\right)$, onde $H_{r+1}^{*} \perp H_{i}, \quad \forall i=1, \ldots, r, r+2, \ldots, n$.

Seguindo [CF04] e [CF05], temos

$$
\rho\left(H_{r+1}^{*}\right)=\sum_{i=1}^{r} \frac{i}{r+1} \rho\left(H_{i}\right)+\rho\left(H_{r+1}\right)+\sum_{j=1}^{n-r-1} \frac{j}{n-r} \rho\left(H_{n-j+1}\right)
$$

Nosso objetivo será encontrar $b_{r+1}^{*}(z)$ tal que

$$
b_{r+1}^{*}(z)=\sum_{i=1}^{r} \frac{i}{r+1} b_{i}(z)+b_{r+1}(z)+\sum_{j=1}^{n-r-1} \frac{j}{n-r} b_{n-j+1}(z)
$$

$\operatorname{com}\left[b_{i}(z), b_{j}(w)\right]=\mathfrak{B}_{i j} \partial_{w} \delta(z / w)$. Desta forma, as relações $\left(R_{1}\right)-\left(R_{6}\right)$ serão satisfeitas. 
Seja

$$
c_{r+1}(z)=-\sum_{i=1}^{r} \frac{i}{r+1} b_{i}(z)-\sum_{j=1}^{n-r-1} \frac{j}{n-r} b_{n-j+1}(z) .
$$

e $b_{r+1}(z)=c_{r+1}(z)+b_{r+1}^{*}(z)$.

Para começar, faremos os cálculos de $c_{r+1}(z)$ considerando cada um dos casos abaixo.

Caso 1) $0<j<r$.

$$
\begin{aligned}
{\left[c_{r+1}(z), b_{j}(w)\right] } & =-\frac{j-1}{r+1}\left[b_{j-1}(z), b_{j}(w)\right]-\frac{j}{r+1}\left[b_{j}(z), b_{j}(w)\right]-\frac{j+1}{r+1}\left[b_{j+1}(z), b_{j}(w)\right] \\
& =\left(-\frac{j-1}{r+1}\left(-\gamma^{2}\right)-\frac{j}{r+1}\left(2 \gamma^{2}\right)-\frac{j+1}{r+1}\left(-\gamma^{2}\right)\right) \partial_{w} \delta(z / w) \\
& =\frac{j \gamma^{2}-\gamma^{2}-2 j \gamma^{2}+j \gamma^{2}+\gamma^{2}}{r+1} \partial_{w} \delta(z / w)=0 \partial_{w} \delta(z / w) \\
& =\mathfrak{B}_{r+1, j} \partial_{w} \delta(z / w) .
\end{aligned}
$$

Caso 2) $j=r$.

$$
\begin{aligned}
{\left[c_{r+1}(z), b_{r}(w)\right] } & =-\frac{r-1}{r+1}\left[b_{r-1}(z), b_{r}(w)\right]-\frac{r}{r+1}\left[b_{r}(z), b_{r}(w)\right] \\
& =\left(-\frac{r-1}{r+1}\left(-\gamma^{2}\right)-\frac{r}{r+1}\left(2 \gamma^{2}\right)\right) \partial_{w} \delta(z / w) \\
& =-\frac{-r \gamma^{2}+\gamma^{2}+2 r \gamma^{2}}{r+1} \partial_{w} \delta(z / w)=-\gamma^{2} \partial_{w} \delta(z / w) \\
& =\mathfrak{B}_{r+1, r} \partial_{w} \delta(z / w) .
\end{aligned}
$$

Caso 3) $j>r+2$.

$$
\begin{aligned}
{\left[c_{r+1}(z), b_{j}(w)\right]=} & -\frac{n-j+2}{n-r}\left[b_{j-1}(z), b_{j}(w)\right]-\frac{n-j+1}{n-r}\left[b_{j}(z), b_{j}(w)\right]-\frac{n-j}{n-r}\left[b_{j+1}(z), b_{j}(w)\right] \\
= & \left(-\frac{n-j+2}{n-r}\left(-\left(\gamma^{2}-(r+1)\right)\right)-\frac{n-j+1}{n-r}\left(2\left(\gamma^{2}-(r+1)\right)\right)\right. \\
& \left.\quad-\frac{n-j}{n-r}\left(-\left(\gamma^{2}-(r+1)\right)\right)\right) \partial_{w} \delta(z / w) \\
= & \frac{\left(\gamma^{2}-(r+1)\right)(n-j+2-2 n+2 j-2+n-j)}{n-r} \partial_{w} \delta(z / w)=0 \partial_{w} \delta(z / w) \\
= & \mathfrak{B}_{r+1, j} \partial_{w} \delta(z / w) .
\end{aligned}
$$

Caso 4) $j=r+2$.

$$
\begin{aligned}
{\left[c_{r+1}(z),\right.} & \left.b_{r+2}(w)\right]=-\frac{n-r-1}{n-r}\left[b_{r+2}(z), b_{r+2}(w)\right]-\frac{n-r-2}{n-r}\left[b_{r+3}(z), b_{r+2}(w)\right] \\
= & \left(-\frac{n-r-1}{n-r}\left(2\left(\gamma^{2}-(r+1)\right)\right)-\frac{n-r-2}{n-r}\left(-\left(\gamma^{2}-(r+1)\right)\right)\right) \partial_{w} \delta(z / w) \\
= & \frac{\left(\gamma^{2}-(r+1)\right)(-2 n+2 r+2+n-r-2)}{n-r} \partial_{w} \delta(z / w)=-\left(\gamma^{2}-(r+1)\right) \partial_{w} \delta(z / w) \\
= & \mathfrak{B}_{r+1, r+2} \partial_{w} \delta(z / w) .
\end{aligned}
$$

Caso 5) $j=r+1$. Temos 


$$
\begin{aligned}
{\left[c_{r+1}(z), c_{r+1}(w)\right] } & =-\frac{r}{r+1}\left[c_{r+1}(z)(z), b_{r}(w)\right]-\frac{n-r-1}{n-r}\left[c_{r+1}(z), b_{r+2}(w)\right] \\
& =\left(-\frac{r}{r+1}\left(-\gamma^{2}\right)-\frac{n-r-1}{n-r}\left(-\gamma^{2}+r+1\right)\right) \partial_{w} \delta(z / w) \\
& \neq 2\left(\gamma^{2}-\frac{r}{2}-1\right) \partial_{w} \delta(z / w)=\mathfrak{B}_{r+1, r+1} \partial_{w} \delta(z / w) .
\end{aligned}
$$

Observe que apenas

$$
\left[c_{r+1}(z), c_{r+1}(w)\right] \neq \mathfrak{B}_{r+1, r+1} \partial_{w} \delta(z / w),
$$

logo basta encontrar $b_{r+1}^{*}(z)$ tal que

$$
\left[b_{r+1}^{*}(z), b_{j}(w)\right]=0, \forall j \neq r+1 .
$$

Para definir $b_{r+1}^{*}(z)$ usaremos

$$
b_{0}(z)=\sum_{m \in \mathbb{Z}} b_{0 m} z^{-m-1}
$$

para nos auxiliar. Seja

$$
K_{1}(z)=\sum_{i=1}^{r+1} \frac{i}{r+1} b_{r-i+1}(z) .
$$

Temos $\left[K_{1}(z), b_{j}(w)\right]=0, \forall j=1, \ldots, r, r+2, \ldots, n$ e, consequentemente,

$$
\left[K_{1}(z), c_{r+1}(w)\right]=0 .
$$

Desta forma,

$$
\begin{aligned}
{\left[K_{1}(z), K_{1}(w)\right] } & =\left[b_{0}(z), b_{0}(w)\right]+\frac{r}{r+1}\left[b_{0}(z), b_{1}(w)\right]=\left(2 \gamma^{2}-\frac{r}{r+1} \gamma^{2}\right) \partial_{w} \delta(z / w) \\
& =\left(\frac{r+2}{r+1} \gamma^{2}\right) \partial_{w} \delta(z / w) .
\end{aligned}
$$

Seja $K(z)=\frac{1}{\gamma} \sqrt{\frac{r+1}{r+2}} K_{1}(z)$. Então $[K(z), K(w)]=\partial_{w} \delta(z / w)$. Agora definamos

$$
b_{r+1}^{*}(z)=\alpha K(z)
$$

para $\alpha \in \mathbb{C}$ tal que

$$
\alpha^{2}=2\left(\gamma^{2}-\frac{r}{2}-1\right)-\left(\frac{r}{r+1} \gamma^{2}+\frac{n-r-1}{n-r} \gamma^{2}-\frac{n-r-1}{n-r}(r+1)\right)
$$

e então

$$
\left[b_{r+1}(z), b_{r+1}(w)\right]=\mathfrak{B}_{r+1, r+1} \partial_{w} \delta(z / w) .
$$

Assim encontramos $b_{r+1}^{*}(z)$ com as propriedades desejadas.

Observação 3.2.4. Precisamos usar $b_{0 m}$ já que não utilizamos $b_{r+1, m}$ de [CF04]. Isto altera a matriz $\mathfrak{B}$ definida nas seções anteriores. 
Observação 3.2.5. Note que os produtos ordenados normais

$$
: a_{i j}(z) a_{i j}^{*}(z):=f_{i j}(z)=\sum_{m \in \mathbb{Z}} f_{i j, m} z^{-m-1}
$$

estão todos bem-definidos, logo também o está $\rho\left(H_{i}\right)(z)$. Temos

$$
\begin{array}{clll}
f_{i j, m} \cdot 1=0, \quad \forall m>0 \quad \text { quando } & j \leqslant r \quad e \\
f_{i j, m} \cdot 1=0, \quad \forall m \in \mathbb{Z} \quad \text { quando } & j \geqslant r+1 .
\end{array}
$$

Por esta razão, temos

$$
\begin{gathered}
\rho\left(H_{i m}\right) \cdot 1=0, \quad \forall m>0 \quad \text { quando } i \leqslant r \quad \text { e } \\
\rho\left(H_{i m}\right) .1=0, \quad \forall m \in \mathbb{J} \quad \text { ou } \quad-m \in \mathbb{N}^{*}-\mathbb{J}, \quad \text { quando } i \geqslant r+2 .
\end{gathered}
$$

Observe que $\rho\left(H_{r+1, m}\right) .1 \neq 0$, mas $\rho\left(H_{r+1, m}^{*}\right) .1=0, \forall m>0$. Agora podemos definir nossa representação para módulos đ-intermediários de Wakimoto.

Teorema 3.2.6. Sejam $\lambda \in \mathfrak{h}^{*}$ e $\lambda_{i}=\lambda\left(H_{i}\right)$. A função geradora

$$
\begin{aligned}
& \rho\left(F_{i}\right)(z)=a_{i i}(z)+\sum_{j=i+1}^{n} a_{i j}(z) a_{i+1, j}^{*}(z), \\
& \rho\left(H_{i}\right)(z)=2: a_{i i}(z) a_{i i}^{*}(z):+\sum_{j=1}^{i-1}\left(: a_{j i}(z) a_{j i}^{*}(z):-: a_{j, i-1}(z) a_{j, i-1}^{*}(z):\right) \\
& +\sum_{j=i+1}^{n}\left(: a_{i j}(z) a_{i j}^{*}(z):-: a_{i+1, j}(z) a_{i+1, j}^{*}(z):\right)+b_{i}(z), \\
& \rho\left(E_{i}\right)(z)=\sum_{k=1}^{i-1}: a_{i i}^{*}(z) a_{k, i-1}(z) a_{k, i-1}^{*}(z):-\sum_{k=1}^{i}: a_{i i}^{*}(z) a_{k i}(z) a_{k i}^{*}(z): \\
& +\sum_{k=i+1}^{n} a_{i+1, k}(z) a_{i k}^{*}(z)-\sum_{k=1}^{i-1} a_{k, i-1}(z) a_{k i}^{*}(z)-a_{i i}^{*}(z) b_{i}(z) \\
& -\left(\delta_{i>r}(r+1)+\delta_{i \leqslant r}(i+1)-\gamma^{2}\right) \partial a_{i i}^{*}(z),
\end{aligned}
$$

define uma ação nos geradores $c, E_{i m}, F_{i m}, H_{i m}, i=1, \ldots, n, m \in \mathbb{Z}$ e $c$, no espaço $\mathbb{C}[\mathbf{x}] \otimes \mathbb{C}_{n}[\mathbf{y}]$.

Precisamos verificar as relações $\left(R_{1}\right)-\left(R_{6}\right)$.

\section{Lema 3.2.7. Temos}

$\left(R_{1}\right)\left[\rho\left(H_{i}\right)(z), \rho\left(H_{j}\right)(w)\right]=\left(\alpha_{i} \mid \alpha_{j}\right) \rho(c) \partial_{w} \delta(z / w)$,

$\left(R_{2}\right)\left[\rho\left(H_{i}\right)(z), \rho\left(E_{j}\right)(w)\right]=\left(\alpha_{i} \mid, \alpha_{j}\right) \rho\left(E_{j}\right)(z) \delta(z / w)$,

$\left(R_{3}\right)\left[\rho\left(H_{i}\right)(z), \rho\left(F_{j}\right)(w)\right]=-\left(\alpha_{i} \mid \alpha_{j}\right) \rho\left(F_{j}\right)(z) \delta(z / w)$,

$\left(R_{4}\right)\left[\rho\left(E_{i}\right)(z), \rho\left(F_{j}\right)(w)\right]=\delta_{i j}\left(\rho\left(H_{i}\right)(z) \delta(z / w)+\rho(c) \partial_{w} \delta(z / w)\right)$,

$\left(R_{5} \mid R_{6}\right)\left[\rho\left(F_{i}\right)(z), \rho\left(F_{j}\right)(w)\right]=\left[\rho\left(E_{i}\right)(z), \rho\left(E_{j}\right)(w)\right]=0$ se $\left(\alpha_{i} \mid \alpha_{j}\right) \neq-1$,

$$
\left[\rho\left(F_{i}\right)\left(z_{1}\right), \rho\left(F_{i}\right)\left(z_{2}\right), \rho\left(F_{j}\right)(w)\right]=\left[\rho\left(E_{i}\right)\left(z_{1}\right), \rho\left(E_{i}\right)\left(z_{2}\right), \rho\left(E_{j}\right)(w)\right]=0,
$$

se $\left(\alpha_{i} \mid \alpha_{j}\right)=-1$. 
Demonstração. A demonstração é similar à feita no Teorema 3.1 .27 para módulos intermediários de Wakimoto já que $\left[b_{i}(z), b_{j}(w)\right]=\mathfrak{B}_{i j} \partial_{w} \delta(z / w)$.

Assim definimos uma realização tipo bóson de $\widehat{\mathfrak{s l}}(n+1, \mathbb{C})$ e uma estrutura de módulo no espaço $\mathbb{C}[\mathbf{x}] \otimes \mathbb{C}_{n}[\mathbf{y}]_{r}$ que depende do subconjunto $\mathbb{J} \subseteq \mathbb{N}^{*}$ e do parâmetro $0 \leqslant r \leqslant n$. Chamamos estes módulos de módulos $\mathbb{J}$-intermediários de Wakimoto e os denotamos por $W_{n, r, \mathbb{J}}(\lambda, \gamma)$.

\subsection{Gerando módulos $\mathbb{J}$-intermediários de Wakimoto}

Nesta seção, estudamos a estrutura de módulos J-intermediários de Wakimoto (lembrando que aqui $\mathbb{C}[\mathbf{y}]$ também significa $\mathbb{C}[\mathbf{y}]_{r}$ ).

Seja $\widehat{\mathfrak{a}}=\widehat{\mathfrak{s l}}(r+1)$ com $\widehat{\mathfrak{a}} \subseteq \widehat{\mathfrak{g}}=\widehat{\mathfrak{s l}}(n+1) . M_{r}(\lambda)$ denota um módulo do tipo Verma com peso máximo $\lambda \mathrm{e}$

$$
W_{\mathfrak{g}, \mathbb{J}}(\lambda)=\mathbb{C}[\mathbf{x}] \otimes \mathbb{C}_{n}[\mathbf{y}]
$$

com a ação definida pelo Teorema 3.2.6. Como em [CF05], onde $\mathfrak{B}$ é não-degenerada, $W_{\mathfrak{g}, \mathbb{J}}(\lambda)$ é o módulo J-intermediário de Wakimoto $W_{n, r, \mathbb{J}}(\lambda, \gamma)$. Para $1 \leq k \leq n$, sejam

$$
\mathbb{C}_{k}[\mathbf{x}]=\mathbb{C}\left[x_{i j, m} \mid 1 \leq i \leq j \leq k, \quad m \in \mathbb{Z}\right]
$$

e

$$
W_{\mathfrak{a}, \mathbb{J}}\left(\lambda_{r}\right)=\mathbb{C}_{r}[\mathbf{x}] \otimes \mathbb{C}_{r}[\mathbf{y}] .
$$

Então $W_{\mathfrak{a}, \mathbb{J}}\left(\lambda_{r}\right) \simeq W$ é um $\widehat{\mathfrak{a}}$-módulo. Mostremos que, de fato, $W_{\mathfrak{g}, \mathbb{J}}(\lambda)$ é gerado por $W_{\mathfrak{a}, \mathbb{J}}\left(\lambda_{r}\right)$.

Teorema 3.3.4. $W_{\mathfrak{g}, \mathbb{J}}(\lambda)=U(\widehat{\mathfrak{g}}) W_{\mathfrak{a}, \mathbb{J}}\left(\lambda_{r}\right)$.

Demonstração. Observe que

$$
L\left(n^{-}(r)\right)=n^{-}(r) \otimes \mathbb{C}\left[t, t^{-1}\right]
$$

é gerado por $F_{i, m}$, com $r<i \leq n$, e $W_{\mathfrak{a}}\left(\lambda_{r}\right)$ é gerado por $x_{i j, m}$ e $e_{j} \cdot y_{p}$, com $1 \leq i \leq j \leq r, m \in \mathbb{Z}$ e $p \in \mathbb{I}$. Para $r \leq k \leq n$, seja

$$
W_{k}=\mathbb{C}_{k}[\mathbf{x}] \otimes \mathbb{C}_{n}[\mathbf{y}]
$$

A idéia é provar que

$$
W_{r} \subseteq U(\widehat{\mathfrak{g}}) W_{\mathfrak{a}, \mathbb{J}}\left(\lambda_{r}\right)
$$

e depois, por indução, mostrar que, para todo $r \leqslant k_{1}<k_{2}$,

$$
W_{k_{1}} \subseteq U(\widehat{\mathfrak{g}}) W_{\mathfrak{a}, \mathbb{J}}\left(\lambda_{r}\right) \Longrightarrow W_{k_{2}} \subseteq U(\widehat{\mathfrak{g}}) W_{\mathfrak{a}, \mathbb{J}}\left(\lambda_{r}\right)
$$

e usando o fato que $W_{n}=W_{\mathfrak{g}, \mathbb{J}}(\lambda)$, o resultado segue.

Mostremos que $W_{r} \subseteq U(\widehat{\mathfrak{g}}) W_{\mathfrak{a}, \mathbb{J}}\left(\lambda_{r}\right)$. Um elemento arbitrário em $W_{r}$ é da forma

$$
\sum_{j} u_{j} \otimes v_{j}, u_{j} \in \mathbb{C}_{r}[\mathbf{x}], v_{j} \in \mathbb{C}_{n}[\mathbf{y}]
$$


e assim é suficiente mostrar que todo elemento da forma $u \otimes v$, com $u \in \mathbb{C}_{r}[\mathbf{x}]$ e $v \in \mathbb{C}_{r}[\mathbf{y}]$ monômios, está em $U(\widehat{\mathfrak{g}}) W_{\mathfrak{a}, \mathbb{J}}\left(\lambda_{r}\right)$. Seja $v=v^{r} v_{r}$, onde

$$
v_{r} \in \mathbb{C}\left[e_{j} \cdot y_{m} \mid 1 \leq j \leq r, m \in \mathbb{N}\right]
$$

e

$$
v^{r} \in \mathbb{C}\left[e_{j} \cdot y_{m} \mid r<j \leq n, m \in \mathbb{N}\right] .
$$

Assuma que

$$
v^{r}=\left(e_{r} \cdot y_{i_{1}}\right)^{p_{i_{1}}} \ldots\left(e_{n} \cdot y_{i_{s}}\right)^{p_{i_{s}}}
$$

é um monômio não-constante (aqui $i_{1}, \ldots, i_{s} \in \mathbb{N}$ ). Seja

$$
\begin{aligned}
\rho\left(H_{i}\right)(z)= & 2: a_{i i}(z) a_{i i}^{*}(z):+\sum_{j=1}^{i-1}\left(: a_{j i}(z) a_{j i}^{*}(z):-: a_{j, i-1}(z) a_{j, i-1}^{*}(z):\right) \\
& +\sum_{j=i+1}^{n}\left(: a_{i j}(z) a_{i j}^{*}(z):-: a_{i+1, j}(z) a_{i+1, j}^{*}(z):\right)+b_{i}(z) .
\end{aligned}
$$

Caso 1) $i>r+1$

Para $m \in \mathbb{J}$ e $-m \in \mathbb{N}^{*} \backslash \mathbb{J}$, os somandos acima possuem fatores com $a_{i k, m}^{*}, k \geqslant r+1$, agindo como zero em $W_{\mathfrak{a}, \mathbb{J}}\left(\lambda_{r}\right)$, já que eles são iguais a $\partial / \partial x_{i k,-m}$, com $k \geqslant r+1$ e $W_{\mathfrak{a}, \mathbb{J}}\left(\lambda_{r}\right)=\mathbb{C}_{r}[\mathbf{x}] \otimes \mathbb{C}_{r}[\mathbf{y}]$. Então temos, quando restrito a $W_{\mathfrak{a}, \mathbb{J}}\left(\lambda_{r}\right)$, que $\rho\left(H_{i m}\right)=b_{i m}$ e este é dado pela multiplicação a esquerda por $e_{i} \cdot y_{m}$, se $m \in \mathbb{J}$, e por $e_{i} \cdot y_{-m}$, se $-m \in \mathbb{N}^{*} \backslash \mathbb{J}$.

Caso 2) $i=r+1$

Neste caso temos, quando restrito à $W_{\mathfrak{a}, \mathbb{J}}\left(\lambda_{r}\right)$,

$$
\rho\left(H_{r+1, m}\right)=-\sum_{j=1}^{r} \sum_{p \in \mathbb{Z}}: a_{j r, p} a_{j r, m-p}^{*}:+b_{r+1, m}
$$

onde, para $m \in \mathbb{J}$ e $-m \in \mathbb{N}^{*} \backslash \mathbb{J}$, temos o primeiro somando aplicando $\mathbb{C}_{r}[\mathbf{x}]$ em si mesmo e o segundo somando sendo multiplicação à esquerda por $e_{i} \cdot y_{m}$ se $m \in \mathbb{J}$, ou por $e_{i} \cdot y_{-m}$ se $-m \in \mathbb{N}^{*} \backslash \mathbb{J}$.

Assim, provemos que $W_{r} \subseteq U(\widehat{\mathfrak{g}}) W_{\mathfrak{a}, \mathbb{J}}\left(\lambda_{r}\right)$.

$$
\begin{aligned}
u \otimes v & =u \otimes\left(e_{0} \cdot y_{i_{1}}\right)^{p_{i_{1}}}\left(e_{r+2} \cdot y_{i_{2}}\right)^{p_{i_{2}}} \ldots\left(e_{n} \cdot y_{i_{s}}\right)^{p_{i_{s}}} v_{r} \\
& =\left(\sum_{l=0}^{r} \alpha_{l} b_{l,\left|i_{1}\right|}+\sum_{l=r+2}^{n} \alpha_{l} b_{l, j_{1}}\right)^{p_{i_{1}}} b_{r+2, j_{2}}^{p_{i_{2}}} \ldots b_{n, j_{s}}^{p_{i_{s}}}\left(u \otimes v_{r}\right) \\
& =\left(\sum_{l=0}^{r} \beta_{l}\left(\rho\left(H_{l,\left|i_{1}\right|}\right)+X_{l,\left|i_{1}\right|}\right)+\sum_{r+2}^{n} \beta_{l}\left(\rho\left(H_{l, j_{1}}\right)+X_{l, j_{1}}\right)+\rho\left(H_{r+1, j_{1}}^{*}\right)-X_{r+1, j_{1}}\right)^{p_{i_{1}}} \\
& \cdot\left(\rho\left(H_{r+2, j_{2}}\right)-X_{r+2, j_{2}}\right)^{p_{i_{2}}} \ldots\left(\rho\left(H_{n, j_{s}}\right)-X_{n, j_{s}}\right)^{p_{i_{s}}}\left(u \otimes v_{r}\right),
\end{aligned}
$$

onde $j_{k}=i_{k}$ se $i_{k} \in \mathbb{J}$, e $j_{k}=-i_{k}$ se $-i_{k} \in \mathbb{N}^{*} \backslash \mathbb{J}$. Então denotando por $\mathcal{H}$ a álgebra de Lie abeliana gerada por $H_{i m}$, com $1 \leqslant i \leqslant n, m \in \mathbb{N}$, temos

$$
u \otimes v \in U(\mathcal{H}) W_{\mathfrak{a}, \mathbb{J}}\left(\lambda_{r}\right)
$$


e assim,

$$
W_{r} \subseteq U(\widehat{\mathfrak{g}}) W_{\mathfrak{a}, \mathbb{J}}\left(\lambda_{r}\right) .
$$

Fixe $r \leq k<n$ e suponha $W_{k} \subseteq U(\widehat{\mathfrak{g}}) W_{\mathfrak{a}, \mathbb{J}}\left(\lambda_{r}\right)$. Provemos que $W_{k+1} \subseteq U(\widehat{\mathfrak{g}}) W_{\mathfrak{a}, \mathbb{J}}\left(\lambda_{r}\right)$. Conside $u \otimes v \in W_{\mathfrak{g}, \mathbb{J}}(\lambda)$ tal que $u=x_{k+1, k+1, m_{1}}^{p} u_{k}$, onde $u_{k} \in \mathbb{C}_{k}[\mathbf{x}], m_{1} \in \mathbb{Z}, p \in \mathbb{N}$ e $v \in \mathbb{C}_{n}[\mathbf{y}]$. Assuma que $W_{k} \subseteq U(\widehat{\mathfrak{g}}) W_{\mathfrak{a}, \mathbb{J}}\left(\lambda_{r}\right)$. De (4.7) para $i>k$, temos que, quando restrito à $W_{k}$,

$$
\rho\left(F_{i m}\right)=x_{i i, m}-\sum_{p \in \mathbb{Z}} \sum_{j=i+1}^{n} x_{i j, p} \partial_{x_{i+1, j, p-m}}
$$

e, como consequência,

$$
\begin{aligned}
\rho\left(F_{k+1, m}^{p}\right)\left(u_{k} \otimes v\right) & =x_{k+1, k+1, m}^{p} u_{k} \otimes v \\
& =u \otimes v
\end{aligned}
$$

para todo $m \in \mathbb{Z}, p \in \mathbb{N}$. Então

$$
u_{k} \otimes v \in U(\widehat{\mathfrak{g}}) W_{\mathfrak{a}, \mathbb{I}}\left(\lambda_{r}\right) \Longrightarrow u \otimes v \in U(\widehat{\mathfrak{g}}) W_{\mathfrak{a}}\left(\lambda_{r}\right) .
$$

Da prova do Teorema 4.5 de [CF04]

$$
\begin{aligned}
{\left[\rho\left(F_{i}\right)(z), \rho\left(F_{j}\right)(w)\right] } & =\left(\delta_{i, j+1} a_{j, j+1}(w)-\delta_{j, i+1} a_{i, i+1}(z)\right) \delta(z / w) \\
& +\left(\delta_{i, j+1} \sum_{q=j+2}^{n} a_{j q}(z) a_{j+2, q}^{*}(z)-\delta_{j, i+1} \sum_{q=i+2}^{n} a_{i q}(z) a_{i+2, q}^{*}(z)\right) \delta(z / w) .
\end{aligned}
$$

Pode-se mostrar por indução que, para $1 \leq j<i$,

$$
\left[\rho\left(F_{i}\right)\left(z_{i}\right), \ldots, \rho\left(F_{j}\right)\left(z_{j}\right)\right]=\left(a_{j i}\left(z_{j}\right)+\sum_{q=i+1}^{n} a_{j q}\left(z_{j}\right) a_{i+1, q}^{*}\left(z_{j}\right)\right) \prod_{q=1}^{i-1} \delta\left(z_{q}-z_{q+1}\right) .
$$

Então, para todo $j<k+1$, temos

$$
\left[\rho\left(F_{k+1}\right)\left(z_{k+1}\right), \ldots, \rho\left(F_{j}\right)\left(z_{j}\right)\right]_{m}=a_{j, k+1, m}+\sum_{q=k+2}^{n} \sum_{p \in \mathbb{Z}} a_{j q, p} a_{k+2, q, m-p}^{*},
$$

onde

$$
a_{k+2, q, m-p}^{*}=-\partial x_{k+2, q, p-m}, \forall k+2 \leqslant q \leqslant n,
$$

age como zero em $W_{k}$. Sabemos que todos os elementos de $W_{k+1}$ são uma combinação linear de monômios $u \otimes v=u_{i-1, m}^{k} u_{k} \otimes v$, onde

$$
u_{i-1, m}^{k}=x_{i-1, k+1, m_{i}}^{p_{i-1}} \ldots x_{k+1, k+1, m_{k+1}}^{p_{k+1}} \text { e } u_{k} \otimes v \in \mathbb{C}_{k}[\mathbf{x}] \otimes \mathbb{C}_{n}[\mathbf{y}]=W_{k}
$$

Temos

$$
\begin{aligned}
u \otimes v & =u_{i-1, m}^{k} u_{k} \otimes v=x_{i-1, k+1, m_{i-1}}^{p_{i-1}} x_{i, k+1, m_{i}}^{p_{i}} \ldots x_{k+1, k+1, m_{k+1}}^{p_{k+1}} u_{k} \otimes v \\
& =\left[\rho\left(F_{k+1}\right)\left(z_{k+1}\right), \ldots, \rho\left(F_{i-1}\right)\left(z_{i-1}\right)\right]_{m_{i-1}}^{p_{i-1}}\left(u_{k} \otimes v\right),
\end{aligned}
$$

onde $\left[\rho\left(F_{k+1}\right)\left(z_{k+1}\right), \ldots, \rho\left(F_{i-1}\right)\left(z_{i-1}\right)\right]_{m_{i-1}}^{p_{i-1}} \in L\left(n^{-}(r)\right)$ e

$$
u_{k} \otimes v \in W_{k} \subseteq U(\widehat{\mathfrak{g}}) W_{\mathfrak{a}, \mathbb{J}}\left(\lambda_{r}\right) .
$$


Isto prova que $W_{k+1} \subseteq U(\widehat{\mathfrak{g}}) W_{\mathfrak{a}, \mathbb{J}}\left(\lambda_{r}\right)$ e então

$$
W_{\mathfrak{g}, \mathbb{J}}(\lambda)=U(\widehat{\mathfrak{g}}) W_{\mathfrak{a}, \mathbb{J}}\left(\lambda_{r}\right) .
$$

Segue imediatamente o seguinte resultado (ver [CF05] para maiores detalhes):

Corolário 3.3.5. Se $\lambda \in \mathcal{H}^{*}$ é geral tal que $M_{r}(\lambda)$ é irredutivel, então $M_{r}(\lambda) \simeq W_{\mathfrak{g}, \mathbb{J}}(\lambda)$.

Observação 3.3.6. Note que $\mathbb{J}_{1} \neq \mathbb{J}_{2} \Longrightarrow W_{n, r, \mathbb{J}_{1}}(\lambda, \gamma) \not W_{n, r, \mathbb{J}_{2}}(\lambda, \gamma)$.

Usando resultados similares aos da seção 6 de [CF05], podemos obter uma descrição da estrutura dos submódulos dos módulos 『-intermediários de Wakimoto.

\subsection{Módulos $\left(\mathbb{J}_{1}, \mathbb{J}_{2}\right)$-intermediários de Wakimoto}

Primeiramente precisamos substituir $\mathbb{C}[\mathbf{y}]$ por

$$
\mathbb{C}[\mathbf{y}]_{(r, j)}=\mathbb{C}\left[y_{i, m} \mid i,-m \in \mathbb{N}^{*}, 0 \leq i \leq n+1, i \neq r+1, j+2\right] .
$$

Analogamente às seções anteriores, usaremos a notação $\mathbb{C}[\mathbf{y}]$ ao invés de $\mathbb{C}[\mathbf{y}]_{(r, j)}$. Definamos $\rho\left(b_{i m}\right)$ da seguinte forma:

Se $0 \leqslant i \leqslant r$, então

$$
\rho\left(b_{i m}\right)= \begin{cases}\lambda_{i}, & \text { se } \quad m=0, \\ m e_{i} \frac{\partial}{\partial y_{m}}, & \text { se } \quad m>0, \\ e_{i} y_{-m}, & \text { se } \quad m<0 .\end{cases}
$$

Se $r+2 \leqslant i \leqslant j+1$, então

$$
\rho\left(b_{i m}\right)= \begin{cases}\lambda_{i}, & \text { se } m=0, \\ m e_{i} \frac{\partial}{\partial y_{m}}, & \text { se } \quad m \in \mathbb{J}_{1}, \\ e_{i} y_{m}, & \text { se } m \in \mathbb{N}^{*} \backslash \mathbb{J}_{1}, \\ -m e_{i} \frac{\partial}{\partial y_{-m},} & \text { se }-m \in \mathbb{N}^{*} \backslash \mathbb{J}_{1}, \\ e_{i} y_{-m}, & \text { se }-m \in \mathbb{J}_{1} .\end{cases}
$$

Finalmente, se $j+3 \leqslant i \leqslant n+1$, então

$$
\rho\left(b_{i m}\right)= \begin{cases}\lambda_{i}, & \text { se } \quad m=0, \\ m e_{i} \frac{\partial}{\partial y_{m}}, & \text { se } \quad m \in \mathbb{J}_{2}, \\ e_{i} y_{m}, & \text { se } \quad m \in \mathbb{N}^{*} \backslash \mathbb{J}_{2}, \\ -m e_{i} \frac{\partial}{\partial y_{-m}}, & \text { se } \quad-m \in \mathbb{N}^{*} \backslash \mathbb{J}_{2}, \\ e_{i} y_{-m}, & \text { se } \quad-m \in \mathbb{J}_{2} .\end{cases}
$$


Aqui abusamos da notação, escrevendo $b_{i m}$ ao invés de $\rho\left(b_{i m}\right)$. Também definimos $b_{r+1, m}$ e $b_{j+2, m}$ de forma similar ao feito para módulos $\mathbb{J}$ - intermediários de Wakimoto. Sendo assim, seja

$$
H_{r+1}^{*}=\left(\sum_{i=1}^{r} \frac{i}{r+1} H_{i}\right)+H_{r+1}+\left(\sum_{l=1}^{j-r-1} \frac{l}{j-r} H_{j-l+1}\right)
$$

e seja

$$
H_{j+1}^{*}=\left(\sum_{i=1}^{j-r-1} \frac{i}{j-r} H_{r+i+1}\right)+H_{j+1}+\left(\sum_{l=1}^{n-j-1} \frac{l}{n-j} H_{n-l+1}\right)
$$

onde $H_{r+1}^{*} \perp H_{i}$ e $H_{j+1}^{*} \perp H_{i}, \forall i \neq r+1, j+1$. Temos

$$
\begin{gathered}
\rho\left(H_{r+1}^{*}\right)=\sum_{i=1}^{r} \frac{i}{r+1} \rho\left(H_{i}\right)+\rho\left(H_{r+1}\right)+\sum_{l=1}^{j-r-1} \frac{l}{j-r} \rho\left(H_{j-l+1}\right) \\
\rho\left(H_{j+1}^{*}\right)=\sum_{i=1}^{j-r-1} \frac{i}{j-r} \rho\left(H_{r+i+1}\right)+\rho\left(H_{j+1}\right)+\sum_{l=1}^{n-j-1} \frac{l}{n-j} \rho\left(H_{n-l+1}\right) .
\end{gathered}
$$

Seja $d_{k}(z)=b_{k}(z)$ se $1 \leqslant k \leqslant r$ e seja $d_{k}(z)=b_{k+1}(z)$ se $r+2 \leqslant k \leqslant n$. Analogamente ao caso dos módulos لJ-intermediários de Wakimoto, definamos

$b_{j+1}(z)=-\left(\sum_{i=1}^{j-r-1} \frac{i}{j-r} b_{r+i+2}(z)\right)-\left(\sum_{l=1}^{n-j-1} \frac{l}{n-j} b_{n-l+2}(z)\right)+\alpha\left(\sum_{p=1}^{j-r} \frac{p}{j-r} b_{j+2-p}(z)\right)$,

onde $\alpha \in \mathbb{C}$ é tal que

$$
\left[d_{j+1}(z), d_{j+1}(w)\right]=\mathfrak{B}_{j+1, j+1} \partial_{w} \delta(z / w) .
$$

Temos

$$
\left[d_{j+1}(z), d_{k}(w)\right]=\mathfrak{B}_{j+1, k} \partial_{w} \delta(z / w), \forall k=1, \ldots, r, r+2, \ldots, n .
$$

Precisamos definir $d_{r+1}(z)$ tal que

$$
\left[d_{j+1}(z), d_{r+1}(w)\right]=\mathfrak{B}_{j+1, r+1} \partial_{w} \delta(z / w) .
$$

Seja

$$
d_{r+1}(z)=-\left(\sum_{i=1}^{r} \frac{i}{r+1} d_{i}(z)\right)-\left(\sum_{l=1}^{n-r-1} \frac{l}{n-r} d_{n-l+1}(z)\right)+\beta\left(\sum_{p=1}^{r+1} \frac{p}{r+1} b_{r+1-p}(z)\right)
$$

onde $\beta \in \mathbb{C}$ é tal que

$$
\left[d_{r+1}(z), d_{r+1}(w)\right]=\mathfrak{B}_{r+1, r+1} \partial_{w} \delta(z / w) .
$$

Temos

$$
\left[d_{r+1}(z), d_{k}(w)\right]=\mathfrak{B}_{j+1, k} \partial_{w} \delta(z / w), \forall k=1, \ldots, n
$$


Teorema 3.4.6. Sejam $\lambda \in \mathfrak{h}^{*}$ e $\lambda_{i}=\lambda\left(H_{i}\right)$. A função dada por

$$
\begin{aligned}
& \rho\left(F_{i}\right)(z)=a_{i i}(z)+\sum_{j=i+1}^{n} a_{i j}(z) a_{i+1, j}^{*}(z), \\
& \rho\left(H_{i}\right)(z)=2: a_{i i}(z) a_{i i}^{*}(z):+\sum_{j=1}^{i-1}\left(: a_{j i}(z) a_{j i}^{*}(z):-: a_{j, i-1}(z) a_{j, i-1}^{*}(z):\right) \\
& +\sum_{j=i+1}^{n}\left(: a_{i j}(z) a_{i j}^{*}(z):-: a_{i+1, j}(z) a_{i+1, j}^{*}(z):\right)+d_{i}(z), \\
& \rho\left(E_{i}\right)(z)=\sum_{k=1}^{i-1}: a_{i i}^{*}(z) a_{k, i-1}(z) a_{k, i-1}^{*}(z):-\sum_{k=1}^{i}: a_{i i}^{*}(z) a_{k i}(z) a_{k i}^{*}(z): \\
& +\sum_{k=i+1}^{n} a_{i+1, k}(z) a_{i k}^{*}(z)-\sum_{k=1}^{i-1} a_{k, i-1}(z) a_{k i}^{*}(z)-a_{i i}^{*}(z) d_{i}(z) \\
& -\left(\delta_{i>r}(r+1)+\delta_{i \leqslant r}(i+1)-\gamma^{2}\right) \partial a_{i i}^{*}(z),
\end{aligned}
$$

onde $d_{i}(z)=b_{i}(z)$, se $i \neq j+1$, define uma ação nos geradores $c, E_{i m}, F_{i m}, H_{i m}$, $i=1, \ldots, n, m \in \mathbb{Z}$ e c, no espaço $\mathbb{C}[\mathbf{x}] \otimes \mathbb{C}_{n}[\mathbf{y}]_{(r, j)}$ que nos dará a estrutura de módulos $\left(\mathbb{J}_{1}, \mathbb{J}_{2}\right)$-intermediários de Wakimoto.

Pode-se checar as relações $\left(R_{1}\right)-\left(R_{6}\right)$ de forma análoga ao do Teorema 3.2.6 para módulos J-intermediários de Wakimoto.

Observação 3.4.7. Temos

$\rho\left(H_{i m}\right) .1=0, \quad \forall m>0 \quad$ quando $i \leqslant r e$

$\rho\left(H_{i m}\right) .1=0, \quad \forall m \in \mathbb{J}_{1} \quad$ ou $\quad-m \in \mathbb{N}^{*}-\mathbb{J}_{1}, \quad$ quando $\quad r+2 \leqslant i \leqslant j e$ $\rho\left(H_{i m}\right) .1=0, \quad \forall m \in \mathbb{J}_{2} \quad$ ou $\quad-m \in \mathbb{N}^{*}-\mathbb{J}_{2}, \quad$ quando $\quad j+2 \leqslant i \leqslant n$.

Note que

(1) $\rho\left(H_{r+1, m}\right) .1 \neq 0$ mas $\rho\left(H_{r+1, m}^{*}\right) \cdot 1=0, \forall m>0$ e

(2) $\rho\left(H_{j+1, m}\right) \cdot 1 \neq 0$ mas $\rho\left(H_{j+1, m}^{*}\right) \cdot 1=0, \forall m \in \mathbb{J}_{1}$ ou $-m \in \mathbb{N}^{*}-\mathbb{J}_{1}$.

Observação 3.4.8. Para inteiros positivos fixados $r_{1}, \ldots, r_{k}$ pode-se definir módulos $\left(\mathbb{J}_{1}, \ldots, \mathbb{J}_{k}\right)$-intermediários de Wakimoto usando basicamente os mesmos argumentos apresentados nesta seção. 


\section{Capítulo 4}

\section{A ação de Virasoro nos módulos imaginários de Wakimoto para $\widehat{\mathfrak{s l}}(2, \mathbb{C})$}

Neste capítulo, nosso objetivo principal é mostrar que os módulos imaginários de Wakimoto, assim como os módulos de Verma imaginários, admitem uma ação de álgebra de Virasoro, como já o admitiam os módulos de Verma clássicos (ver [EFK98]). Para tal propósito, definimos primeiramente para $m \in \mathbb{Z}$, operadores auxiliares $\bar{L}_{m}$ e, posteriormente, $L_{m}$ que agem em nossos módulos.

Depois algumas definições auxiliares se fazem necessárias como, por exemplo, o conceito de módulo topológico, de completamento e de limite inverso, definições estas que o leitor poderá encontrar em [War93]. Feito isto, introduz-se uma equação análoga à de KnizhnikZamolodchikov feita em [EFK98] para os módulos de Verma clássicos e, usando o fato de que os módulos de Verma imaginários são genericamente isomorfos aos módulos de Wakimoto imaginários (confrontar Observação 1.4.15), finalizamos nosso trabalho com o análogo desta equação para os módulos imaginários de Wakimoto

Vale ressaltar que os resultados obtidos neste capítulo referem-se ao artigo [CFMar12], em que somente levamos em consideração a álgebra $\widehat{\mathfrak{s l}}(2, \mathbb{C})$, e não $\widehat{\mathfrak{s l}}(n, \mathbb{C})$, como nos capítulos anteriores. Acreditamos que, neste caso, o resultado para módulos intermediários de Wakimoto sejam válidos (neste capítulo abordamos apenas os imaginários pois estamos em $\widehat{\mathfrak{s l}}(2, \mathbb{C}))$, sendo que o estudo para $\widehat{\mathfrak{s l}}(n, \mathbb{C})$ será feito em pesquisas futuras.

\subsection{Introdução}

Seja $e=e_{12}, f=e_{21}$ e $h=e_{11}-e_{22}$ a base usual de $\mathfrak{g}=\mathfrak{s l}(2, \mathbb{C})$, onde $e_{i j}$ é a base canônica de $\mathfrak{g l}(2, \mathbb{C})$. Temos

$$
\mathfrak{g}=\mathfrak{n}^{+} \oplus \mathfrak{h} \oplus \mathfrak{n}^{-}
$$

onde $\mathfrak{n}^{+}=\mathbb{C} e, \mathfrak{h}=\mathbb{C} h$ e $\mathfrak{n}^{-}=\mathbb{C} f$. Defina

$$
\widehat{\mathfrak{g}}=\widehat{\mathfrak{s l}}(2, \mathbb{C})=\left(\mathfrak{g} \otimes \mathbb{C}\left[t, t^{-1}\right]\right) \oplus \mathbb{C} c=\widehat{\mathfrak{n}}^{+} \oplus \widehat{\mathfrak{h}} \oplus \widetilde{\mathfrak{n}}^{-}
$$

onde

$$
\widehat{\mathfrak{n}}^{+}=\mathfrak{n}^{+} \otimes \mathbb{C}\left[t, t^{-1}\right] \oplus \mathfrak{h} \otimes t \mathbb{C}[t],
$$




$$
\begin{gathered}
\widehat{\mathfrak{h}}=\mathfrak{h} \oplus \mathbb{C} c, \\
\widehat{\mathfrak{n}}^{-}=\mathfrak{n}^{-} \otimes \mathbb{C}\left[t, t^{-1}\right] \oplus \mathfrak{h} \otimes t^{-1} \mathbb{C}\left[t^{-1}\right] .
\end{gathered}
$$

A álgebra $\widehat{\mathfrak{g}}$ é gerada pelos elementos $e_{m}, f_{m}, h_{m}$, onde $m \in \mathbb{Z}$, e pelo elemento central $c$, com o produto

$$
\left[X_{m}, Y_{n}\right]=[X, Y]_{m+n}+\delta_{m+n, 0} m(X \mid Y) c,
$$

onde $X_{m}=X \otimes t^{m}$, para $X, Y \in \mathfrak{g}$ e $m \in \mathbb{Z}$. Seja

$$
(X \mid Y)=\operatorname{tr}(X Y)
$$

a forma de Killing. Defina

$$
\widetilde{\mathfrak{g}}=\widehat{\mathfrak{g}} \oplus \mathbb{C} d=\widehat{\mathfrak{n}}^{+} \oplus \widetilde{\mathfrak{h}} \oplus \widehat{\mathfrak{n}}^{-},
$$

onde $\widetilde{\mathfrak{h}}=\widehat{\mathfrak{h}} \oplus \mathbb{C} d,\left[d, X_{m}\right]=m X_{m}$ e $[d, c]=0$. A subálgebra $\mathfrak{b}_{\text {nat }}=\widetilde{\mathfrak{h}} \oplus \widehat{\mathfrak{n}}^{+}$é a subálgebra de Borel natural introduzida por Jakobsen e Kac em [JK85]. Em $\widetilde{\mathfrak{h}}$, temos o espaço dual $\widetilde{\mathfrak{h}}^{*}$ tal que

$$
\widetilde{\mathfrak{h}}^{*}=\mathfrak{h}^{*} \oplus \mathbb{C} \Lambda_{0} \oplus \mathbb{C} \delta
$$

onde, para todo $h \in \mathfrak{h}$,

$$
\begin{gathered}
\Lambda_{0}(c)=1, \Lambda_{0}(d)=\Lambda_{0}(h)=0, \\
\delta(d)=1, \delta(c)=\delta(h)=0 .
\end{gathered}
$$

Denotemos por $\Delta$ o sistema de raízes de $\widetilde{\mathfrak{g}}$, e seja $\left\{\alpha_{0}, \alpha_{1}\right\}$ uma base de $\Delta$. Então $\delta=\alpha_{0}+\alpha_{1}$ é a raiz imaginária positiva e indivisível e

$$
\Delta=\left\{ \pm \alpha_{1}+n \delta \mid n \in \mathbb{Z}\right\} \cup\{k \delta \mid k \in \mathbb{Z} \backslash\{0\}\} .
$$

Definição 4.1.8. Seja $\Lambda \in \widetilde{\mathfrak{h}}^{*}$ um peso e seja $\mathbb{C}_{\Lambda}$ uma representação unidimensional de $\widetilde{\mathfrak{h}} \oplus \widehat{\mathfrak{n}}^{+}$tal que $\mathbb{C}_{\Lambda}$ é gerada por um vetor $v_{\Lambda}, \widehat{\mathfrak{n}}^{+}$age como zero e $\widetilde{h} v_{\Lambda}=\Lambda(\widetilde{h}) v_{\Lambda}$, para todo $\widetilde{h} \in \widetilde{\mathfrak{h}}$. O módulo de Verma imaginário pode ser definido da seguinte forma (ver [Fut94], [Cox94]):

$$
V_{\Lambda}=\operatorname{In} d_{\widetilde{\mathfrak{h}} \oplus \widehat{\mathfrak{n}}^{+}}^{\widetilde{\mathfrak{g}}} \mathbb{C}_{\Lambda}
$$

Quando uma representação $V$ de $\widetilde{\mathfrak{g}}$ é gerada por um vetor $v_{\Lambda}$ de peso $\Lambda$ que é aniquilado por $\widehat{\mathfrak{n}}^{+}$, dizemos que $V$ tem peso máximo (imaginário) $\Lambda$. Para todo peso $\Lambda$ podemos escrever

$$
\Lambda=\lambda+\kappa \Lambda_{0}-\Delta \delta, \lambda \in \mathfrak{h}^{*} \text { e } \kappa, \Delta \in \mathbb{C} .
$$

onde, por (4.1.7), temos $\lambda(h)=\Lambda(h), \kappa=\Lambda(c)$ e $\Delta=-\Lambda(d)$.

Observação 4.1.11. Daqui em diante consideraremos apenas pesos $\Lambda$ tais que

$$
\Delta=\Delta(\lambda)=\frac{\langle\lambda, \lambda+2 \rho\rangle}{2\left(k+h^{v}\right)}
$$

e, para este $\Lambda$, denotaremos $V_{\lambda, \kappa}$ ao invés de $V_{\Lambda}$. Quando $\kappa$ e $\Delta$ estão fixados chamaremos $V_{\lambda, \kappa}$ de módulo imaginário de peso máximo com peso máximo imaginário $\lambda$. 
Seja

$$
H=(\mathfrak{h} \otimes \mathbb{C}[t] t) \oplus\left(\mathfrak{h} \otimes \mathbb{C}\left[t^{-1}\right] t^{-1}\right) \oplus \mathbb{C} c
$$

uma subálgebra de Heisenberg de $\widetilde{\mathfrak{g}}$. Seja $V_{\lambda}$ o $H$-módulo de Verma gerado por $v_{\Lambda}$. Então

$$
V_{\lambda, \kappa}=\operatorname{Ind}_{H+\mathfrak{b}_{n a t}}^{\widetilde{\mathfrak{g}}} V_{\lambda}
$$

onde $d$ age em $V_{\lambda}$ por multiplicação por $-\Delta(\lambda)$. Em $V_{\lambda, \kappa}$ temos uma $\mathbb{Z}$-graduação:

$$
V_{\lambda, \kappa}=\bigoplus_{n \geq 0} V_{\lambda, \kappa}[-n]
$$

Observe que $V_{\lambda, \kappa}[-n]$ é o autoespaço de $d$ de autovalor $-n-\Delta(\lambda)$, com cada $V_{\lambda, \kappa}[-n]$ sendo um $H$-módulo e $V_{\lambda, \kappa}[0]=V_{\lambda}$.

Observação 4.1.14. Seja $V$ um $\mathfrak{g}$-módulo e fixe $z \in \mathbb{C}^{*}$. Para todo polinômio $P(t) \in \mathbb{C}[t]$ e $x \in \widehat{\mathfrak{g}}, u \in V$, seja

$$
x \otimes P(t) \cdot u=P(z) x u, c u=0 .
$$

Esta é chamada de representação de evaluação de $\widehat{\mathfrak{g}}$ e a denotemos por $V(z)$.

Infelizmente não podemos estendê-la a uma ação de $\widetilde{\mathfrak{g}}$. Seja $\Delta$ o conveniente número complexo que definiremos na Proposição 4.3 .13 e seja $z$ uma variável formal. Considere o espaço

$$
z^{-\Delta} V\left[z, z^{-1}\right]=V \otimes z^{-\Delta} \mathbb{C}\left[z, z^{-1}\right] .
$$

Este espaço possui uma estrutura de módulo em $\tilde{\mathfrak{g}}$, onde $d$ age como $z \frac{\partial}{\partial z}$. Para todo $z_{0} \neq 0$, temos a aplicação de evaluação

$$
\epsilon_{z_{0}}: z^{-\Delta} V\left[z, z^{-1}\right] \longrightarrow V\left(z_{0}\right)
$$

que é um $\widehat{\mathfrak{g}}$-epimorfismo. Denote por

$$
V_{\lambda, \kappa} \widehat{\otimes} V\left(z_{0}\right)
$$

o produto tensorial completo gerado por todas as somas infinitas da forma $\sum_{i=1}^{\infty} w_{i} \otimes v_{i}$, onde $w_{i} \in V_{\lambda, \kappa}$, é um vetor homogêneo, $\left\{\operatorname{degree}\left(w_{i}\right)\right\} \longrightarrow-\infty$ e $v_{i} \in V\left(z_{0}\right), \forall i \in \mathbb{N}^{*}$.

Aqui iremos reescrever a primeira realização de campos livres em termos de campos.

Definição 4.1.18. Seja $\widehat{\mathfrak{a}}$ uma álgebra de Heisenberg de dimensão infinita gerada por $a_{m}$, $a_{m}^{*}$ e $\mathbf{1}$, com $m \in \mathbb{Z}$ e com as relações

$$
\begin{aligned}
{\left[a_{m}, a_{n}\right] } & =\left[a_{m}^{*}, a_{n}^{*}\right]=0, \\
{\left[a_{m}, a_{n}^{*}\right] } & =\delta_{m+n, 0} \mathbf{1}, \\
{\left[a_{m}, \mathbf{1}\right] } & =\left[a_{m}^{*}, \mathbf{1}\right]=0 .
\end{aligned}
$$

Esta álgebra age em $\mathbb{C}\left[x_{m} \mid m \in \mathbb{Z}\right]$ por

$$
a_{m} \mapsto x_{m}, \quad a_{m}^{*} \mapsto-\partial / \partial x_{-m}
$$

com 1 agindo como a identidade. Então temos um $\widehat{\mathfrak{a}}$-módulo gerado por $v$ tal que

$$
a_{m}^{*} v=0, \quad \forall m \in \mathbb{Z} .
$$


Observe que $a(z)$ não é um campo enquanto que $a^{*}(z)$ é. Chamemos este operador $a(z)$ (respectivamente $a^{*}(z)$ ) de operador de criação puro (respectivamente aniquilação) . Seja

$$
\begin{aligned}
a(z)_{+} & =a(z), \quad a(z)_{-}=0, \\
a^{*}(z)_{+} & =0, \quad a^{*}(z)_{-}=a^{*}(z) .
\end{aligned}
$$

Definição 4.1.19. Seja e $(z)=\sum_{n \in \mathbb{Z}} e_{n} z^{-n-1}, f(z)=\sum_{n \in \mathbb{Z}} f_{n} z^{-n-1}$ e $h(z)=\sum_{n \in \mathbb{Z}} h_{n} z^{-n-1}$.

Teorema 4.1.20. ([CF05]). Seja $\lambda \in \mathfrak{H}^{*}, \gamma \in \mathbb{C}$. As funções geradoras

$$
\begin{aligned}
& f(z) \mapsto a(z) \\
& h(z) \mapsto 2: a(z) a^{*}(z):+b(z), \\
& e(z) \mapsto: a^{*}(z)^{2} a(z):-a^{*}(z) b(z)-\left(1-\gamma^{2}\right) \partial_{z} a^{*}(z), \\
& c \mapsto \gamma^{2}-1,
\end{aligned}
$$

definem uma representação de $\widehat{\mathfrak{s l}}(2)$ no espaço de Fock $\mathbb{C}[\mathbf{x}] \otimes \mathbb{C}[\mathbf{y}]$.

Em outras palavras, a primeira e a segunda realização de campos livres podem ser obtidas pela mesma fórmula de Wakimoto tomando diferentes representações da álgebra de Heisenberg $\widehat{\mathfrak{a}}$. Denotemos este $\widehat{\mathfrak{s l}}(2)$-módulo por $W_{\lambda, \kappa}$, onde $\kappa=\gamma^{2}-1$ e o chamemos de módulo imaginário de Wakimoto. Podemos olhar $W_{\lambda, \kappa}$ como um $\widetilde{\mathfrak{s l}}(2, \mathbb{C})$-módulo definindo

$$
d \cdot a_{n_{1}} \cdots a_{n_{k}} b_{-m_{1}} \cdots b_{-m_{l}} \cdot 1=\left(\sum_{j=1}^{k} n_{k}-\sum_{i=1}^{l} m_{i}-\Delta(\lambda)\right) a_{n_{1}} \cdots a_{n_{k}} b_{-m_{1}} \cdots b_{-m_{l}} \cdot 1
$$

onde, em particular, $d \cdot 1=-\Delta(\lambda) \cdot 1$. Denotemos por $w_{\lambda, \kappa}$ o gerador 1 de $W_{\lambda, \kappa}$.

Teorema 4.1.22 ([CF05]). Fixemos $\Lambda$ como em (4.1.10). Para $\kappa=\gamma^{2}-1 \neq 0$, a aplicação $\Psi_{\lambda, \kappa}: V_{\lambda, \kappa} \longrightarrow W_{\lambda, \kappa}$ dada por

$$
\Psi_{\lambda, \kappa}\left(f_{n_{1}} \cdots f_{n_{k}} h_{-m_{1}} \cdots h_{-m_{l}} \cdot v_{\lambda, \kappa}\right)=a_{n_{1}} \cdots a_{n_{k}} b_{-m_{1}} \cdots b_{-m_{l}} \cdot w_{\lambda, \kappa}
$$

é um isomorfismo de $\widetilde{\mathfrak{s l}}(2, \mathbb{C})$-módulos.

\subsection{A ação de Virasoro}

Nosso primeiro objetivo é definir uma ação da álgebra de Virasoro no módulo de Verma imaginário para posteriormente poder definí-la nos módulos imaginários de Wakimoto. Para isso seguiremos a construção feita em [KR87]. A álgebra de Virasoro é definida tendo base $L_{n}, n \in \mathbb{Z}$ e centro gerado por $c$ com as relações

$$
\left[L_{m}, L_{n}\right]=(m-n) L_{m+n}+\frac{1}{12}\left(m^{3}-m\right) \delta_{m+n, 0} c .
$$

Agora defina o operador

$$
\bar{L}_{k}=\sum_{j \in \mathbb{Z}}(j-k) a_{j} a_{k-j}^{*}
$$


o qual agirá no módulo de Wakimoto imaginário. Seja $\psi: \mathbb{R} \rightarrow\{0,1\}$ dada por

$$
\psi(x)= \begin{cases}1 & \text { se }|x| \leq 1 \\ 0 & \text { se }|x|>1\end{cases}
$$

$\mathrm{e}$

$$
\bar{L}_{k}(\epsilon)=\sum_{j \in \mathbb{Z}}(j-k) a_{j} a_{k-j}^{*} \psi(\epsilon j) .
$$

Assim $\bar{L}_{n}(\epsilon)$ possue apenas uma quantidade finita de somandos e como $\epsilon \rightarrow 0$, temos que $\bar{L}_{n}(\epsilon) \rightarrow \bar{L}_{n}$.

Lema 4.2.3. Temos que $\left[a_{k}, \bar{L}_{n}\right]=k a_{k+n} e\left[a_{k}^{*}, \bar{L}_{n}\right]=(k+n) a_{k+n}^{*}$.

Demonstração. De fato, temos que

$$
\left[a_{k}, \bar{L}_{n}(\epsilon)\right]=\sum_{j \in \mathbb{Z}}(j-n)\left[a_{k}, a_{j} a_{n-j}^{*}\right] \psi(\epsilon j)=k a_{k+n} \psi(\epsilon(n+k))
$$

e também que

$$
\left[a_{k}^{*}, \bar{L}_{n}(\epsilon)\right]=\sum_{j \in \mathbb{Z}}(j-n)\left[a_{k}^{*}, a_{j} a_{n-j}^{*}\right] \psi(\epsilon j)=(k+n) a_{k+n}^{*} \psi(\epsilon(-k)) .
$$

Lema 4.2.4. $\left[\bar{L}_{m}, \bar{L}_{n}\right]=(m-n) \bar{L}_{m+n}$.

Demonstração. Temos que

$$
\begin{aligned}
& {\left[\bar{L}_{m}(\epsilon), \bar{L}_{n}\right]=\sum_{j \in \mathbb{Z}}(j-m)\left[a_{j} a_{m-j}^{*}, \bar{L}_{n}\right] \psi(\epsilon j)=\sum_{j \in \mathbb{Z}}(j-m)\left[a_{j}, \bar{L}_{n}\right] a_{m-j}^{*} \psi(\epsilon j)} \\
& \quad+\sum_{j \in \mathbb{Z}}(j-m) a_{j}\left[a_{m-j}^{*}, \bar{L}_{n}\right] \psi(\epsilon j) \\
& =\sum_{j \in \mathbb{Z}}(j-m) j a_{j+n} a_{m-j}^{*} \psi(\epsilon j)+\sum_{j \in \mathbb{Z}}(j-m)(m+n-j) a_{j} a_{m+n-j}^{*} \psi(\epsilon j) \\
& =\sum_{j \in \mathbb{Z}}(j-n-m)(j-n) a_{j} a_{m+n-j}^{*} \psi(\epsilon(j-n))+\sum_{j \in \mathbb{Z}}(j-m)(m+n-j) a_{j} a_{m+n-j}^{*} \psi(\epsilon j) \\
& =\sum_{j \in \mathbb{Z}}((j-n-m)(j-n) \psi(\epsilon(j-n))+(j-m)(m+n-j) \psi(\epsilon j)) a_{j} a_{m+n-j}^{*} .
\end{aligned}
$$

Para $\epsilon$ suficientemente pequeno e $j$ fixado, temos

$$
\begin{aligned}
(j-n-m) & (j-n) \psi(\epsilon(j-n))+(j-m)(m+n-j) \psi(\epsilon j) \\
& =(j-n-m)(j-n)+(j-m)(m+n-j) \\
& =(m-n)(j-m-n) .
\end{aligned}
$$

e então

$$
\left[\bar{L}_{m}, \bar{L}_{n}\right]=(m-n) \bar{L}_{m+n}
$$

Desta forma, o operador $\bar{L}_{n}, n \in \mathbb{Z}$, define uma estrutura de álgebra de Witt, mas não a de Virasoro, como desejamos. Contudo, antes de continuar, escreveremos tal operador na linguagem de distribuições formais. 
Lema 4.2.5. Seja $\bar{L}(z)=\sum_{n \in \mathbb{Z}} \bar{L}_{n} z^{-n-2}$. Temos

$$
\begin{aligned}
& {[\bar{L}(z), \bar{L}(w)]=\sum_{k \in \mathbb{Z}} \sum_{q \in \mathbb{Z}}\left[\bar{L}_{k}, \bar{L}_{q}\right] z^{-k-2} w^{-q-2}} \\
& \quad=\sum_{k \in \mathbb{Z}} \sum_{q \in \mathbb{Z}}(k-q) \bar{L}_{k+q} z^{-k-2} w^{-q-2}=\sum_{l \in \mathbb{Z}} \sum_{q \in \mathbb{Z}}(l-2 q) \bar{L}_{l} z^{-l+q-2} w^{-q-2} \\
& \quad=\left(\sum_{l \in \mathbb{Z}}(l+2) \bar{L}_{l} z^{-l-3}\right)\left(\sum_{q \in \mathbb{Z}} z^{q+1} w^{-q-2}\right)+\left(\sum_{l \in \mathbb{Z}} \bar{L}_{l} z^{-l-2}\right)\left(\sum_{q \in \mathbb{Z}}(-2 q-2) z^{q} w^{-q-2}\right) \\
& \quad=-\partial_{z} \bar{L}(z) \delta(z / w)+2 \bar{L}(z) \partial_{w} \delta(z / w) \\
& \quad=\partial_{w} \bar{L}(w) \delta(z / w)+2 \bar{L}(w) \partial_{w} \delta(z / w) .
\end{aligned}
$$

Agora definiremos o ador que nos dará a ação de álgebra de Virasoro.

Definição 4.2.6. Fixemos $\mu \in \mathbb{C}$. Definamos $L_{k}$ tal que

$$
L(z)=\bar{L}(z)+\frac{1}{4}: b(z)^{2}:+\frac{\mu}{2} \partial_{z} b(z) .
$$

Temos

$$
\sum_{k \in \mathbb{Z}} L_{k} z^{-k-2}=\sum_{k \in \mathbb{Z}}\left(\sum_{j \in \mathbb{Z}}(j-k) a_{j} a_{k-j}^{*}+\frac{1}{4} \sum_{j \in \mathbb{Z}}: b_{j} b_{k-j}:-\frac{\mu}{2}(k+1) b_{k}\right) z^{-k-2}
$$

e assim

$$
L_{k}=\sum_{j \in \mathbb{Z}}(j-k) a_{j} a_{k-j}^{*}+\frac{1}{4} \sum_{j \in \mathbb{Z}}: b_{j} b_{k-j}:-\frac{\mu}{2}(k+1) b_{k}
$$

onde

$$
\sum_{n \in \mathbb{Z}}: b_{n} b_{m-n}:=\sum_{n>m} b_{m-n} b_{n}+\sum_{n \leq m} b_{n} b_{m-n}
$$

já que

$$
\begin{aligned}
: b(z) b(z): & =b(z)_{+} b(z)+b(z) b(z)_{-} \\
& =\sum_{n \in \mathbb{Z}} \sum_{m<0} b_{m} b_{n} z^{-m-n-2}+\sum_{n \in \mathbb{Z}} \sum_{m \geq 0} b_{n} b_{m} z^{-m-n-2} \\
& =\sum_{n \in \mathbb{Z}} \sum_{m<0} b_{m} b_{n-m} z^{-n-2}+\sum_{n \in \mathbb{Z}} \sum_{m \geq 0} b_{n-m} b_{m} z^{-n-2} \\
& =\sum_{n \in \mathbb{Z}} \sum_{m<n} b_{m-n} b_{n} z^{-n-2}+\sum_{n \in \mathbb{Z}} \sum_{m \geq n} b_{n} b_{m-n} z^{-n-2}
\end{aligned}
$$

O centro da álgebra de Virasoro age como escalar $\phi=6-6 \mu^{2} \in \mathbb{C}$. Temos que $b_{i}$ satisfaz a relação

e, portanto,

$$
\left[b_{m}, b_{p}\right]=2 m \delta_{m+p, 0}
$$

$$
[b(z), b(w)]=2 \partial_{w} \delta(z / w)
$$

e

$$
b(z) b(w)=: b(z) b(w):+\frac{2}{(z-w)^{2}} .
$$


Proposição 4.2.11. Seja $b_{0} v=\lambda v, c v=\phi v$ onde $v$ é o vetor vácuo. Então

$$
[L(z), L(w)]=\frac{c}{12} \partial_{w}^{3} \delta(z / w)+2 L(w) \partial_{w} \delta(z / w)+\partial_{w} L(w) \delta(z / w) .
$$

e então, temos que $\bar{L}_{n}, n \in \mathbb{Z}$, satisfaz (4.2.1), nos dando a ação de álgebra de Virasoro no módulo de Verma imaginário, como queríamos.

Demonstração. Faremos estes cálculos usando os Teoremas de Kac, Wick e Taylor (ver [Kac98]). Temos

$$
\begin{aligned}
: b(z)^{2}:: b(w)^{2} & =: b(z)^{2} b(w)^{2}:+\frac{8}{(z-w)^{2}}: b(z) b(w):+\frac{8}{(z-w)^{4}} \\
& =: b(z)^{2} b(w)^{2}:+\frac{8}{(z-w)^{2}}: b(w) b(w):+\frac{8}{(z-w)}: b(w) \partial_{w} b(w):+\frac{8}{(z-w)^{4}},
\end{aligned}
$$

$\log 0$

$$
\left[\frac{1}{4}: b(z)^{2}:, \frac{1}{4}: b(w)^{2}:\right]=2\left(\frac{1}{4}\right): b(w) b(w): \partial_{w} \delta(z / w)+\frac{1}{2}: b(w) \partial_{w} b(w):+\frac{1}{2} \partial_{w}^{3} \delta(z / w) .
$$

Agora temos

$$
: b(z)^{2}: b(w)=: b(z)^{2} b(w):+\frac{4}{(z-w)^{2}} b(w)+\frac{4}{z-w} \partial_{w} b(w)
$$

e, portanto,

$$
\begin{aligned}
& {\left[\frac{1}{4}: b(z)^{2}:, \frac{\mu}{2} \partial_{w} b(w)\right]+\left[\frac{\mu}{2} \partial_{z} b(z), \frac{1}{4}: b(w)^{2}:\right]} \\
& =\partial_{w}\left(\frac{\mu}{2} b(w) \partial_{w} \delta(z / w)+\frac{\mu}{2} \partial_{w} b(w) \delta(z / w)\right)+\partial_{z}\left(\frac{\mu}{2} b(z) \partial_{z} \delta(z / w)+\frac{\mu}{2} \partial_{z} b(z) \delta(z / w)\right) \\
& =\frac{2 \mu}{2} \partial_{w} b(w) \partial_{w} \delta(z / w)+\frac{\mu}{2} \partial_{w}^{2} b(w) \delta(z / w)+\frac{\mu}{2} b(w) \partial_{w}^{2} \delta(z / w)-\frac{\mu}{2} \partial_{z}^{2}(b(z) \delta(z / w)) \\
& =\mu \partial_{w} b(w) \partial_{w} \delta(z / w)+\frac{\mu}{2} \partial_{w}^{2} b(w) \delta(z / w)+\frac{\mu}{2} b(w) \partial_{w}^{2} \delta(z / w)-\frac{\mu}{2} \partial_{z}^{2}(b(w) \delta(z / w)) \\
& =\mu \partial_{w} b(w) \partial_{w} \delta(z / w)+\frac{\mu}{2} \partial_{w}^{2} b(w) \delta(z / w)+\frac{\mu}{2} b(w) \partial_{w}^{2} \delta(z / w)-\frac{\mu}{2} b(w) \partial_{z}^{2} \delta(z / w) \\
& =\mu \partial_{w} b(w) \partial_{w} \delta(z / w)+\frac{\mu}{2} \partial_{w}^{2} b(w) \delta(z / w)+\frac{\mu}{2} b(w) \partial_{w}^{2} \delta(z / w)-\frac{\mu}{2} b(w) \partial_{w}^{2} \delta(z / w) .
\end{aligned}
$$

Para finalizar, observe que $\left[\frac{\mu}{2} \partial_{z} b(z), \frac{\mu}{2} \partial_{w} b(w)\right]=-\frac{\mu^{2}}{2} \partial_{w}^{3} \delta(z / w)$. Juntando todas estas expressões, temos que

$$
\begin{aligned}
{[L(z), L(w)] } & =\partial_{w} \bar{L}(w) \delta(z / w)+2 \bar{L}(w) \partial_{w} \delta(z / w)+\frac{1}{2}: b(w) b(w): \partial_{w} \delta(z / w) \\
& +\frac{1}{2}: b(w) \partial_{w} b(w):+\frac{1}{2} \partial_{w}^{3} \delta(z / w)+\mu \partial_{w} b(w) \partial_{w} \delta(z / w)+\frac{\mu}{2} \partial_{w}^{2} b(w) \delta(z / w) \\
& +\frac{\mu}{2} b(w) \partial_{w}^{2} \delta(z / w)-\frac{\mu}{2} b(w) \partial_{w}^{2} \delta(z / w)-\frac{\mu^{2}}{2} \partial_{w}^{3} \delta(z / w) \\
= & \left(\partial_{w} \bar{L}(w)+\frac{1}{2}: b(w) \partial_{w} b(w):+\frac{\mu}{2} \partial_{w}^{2} b(w)\right) \delta(z / w) \\
& +\left(2 \bar{L}(w)+\frac{1}{2}: b(w)^{2}:+\frac{\mu}{2} \partial_{w} b(w)\right) \partial_{w} \delta(z / w)+\left(\frac{1}{2}-\frac{\mu^{2}}{2}\right) \partial_{w}^{3} \delta(z / w) \\
= & \partial_{w} L(w) \delta(z / w)+2 L(w) \partial_{w} \delta(z / w)+\frac{c}{12} \partial_{w}^{3} \delta(z / w) .
\end{aligned}
$$


Proposição 4.2.13. Temos os seguintes colchetes:

$$
\begin{gathered}
{[a(z), L(w)]=a(w) \partial_{w} \delta(z / w),} \\
{\left[a^{*}(z), L(w)\right]=-\partial_{w} a^{*}(w) \delta(z / w),} \\
{[b(z), L(w)]=b(w) \partial_{w} \delta(z / w)+\mu \partial_{w}^{2} \delta(z / w) .}
\end{gathered}
$$

Demonstração. Temos

$$
\begin{aligned}
& {[a(z), L(w)]=[a(z), \bar{L}(w)]=\sum_{k, n \in \mathbb{Z}}\left[a_{k}, \bar{L}_{n}\right] z^{-k-1} w^{-n-2}=\sum_{k, n \in \mathbb{Z}} k a_{k+n} z^{-k-1} w^{-n-2}} \\
& =\sum_{k, n \in \mathbb{Z}} k a_{k+n} z^{-k-1} w^{k-1} w^{-n-k-1}=\sum_{k, n \in \mathbb{Z}} k a_{n-k} z^{k-1} w^{-k-1} w^{-n+k-1} \\
& =a(w) \partial_{w} \delta(z / w), \\
& {\left[a^{*}(z), L(w)\right]=\left[a^{*}(z), \bar{L}(w)\right]=\sum_{k, n \in \mathbb{Z}}\left[a_{k}^{*}, \bar{L}_{n}\right] z^{-k} w^{-n-2}=\sum_{k, n \in \mathbb{Z}}(k+n) a_{k+n}^{*} z^{-k} w^{-n-2}} \\
& =\sum_{k, n \in \mathbb{Z}}(k+n) a_{k+n}^{*} z^{-k} w^{k} w^{-1} w^{-n-k-1}=-\partial_{w} a^{*}(w) \delta(z / w),
\end{aligned}
$$

e, utilizando os cálculos da Proposição 4.2.11, temos que

$$
\begin{aligned}
{[b(z), L(w)] } & =\left[b(z), \frac{1}{4}: b(w)^{2}:+\frac{\mu}{2} \partial_{w} b(w)\right] \\
& =\frac{1}{4}\left(-4 \partial_{z} b(z) \delta(z / w)-4 b(z) \partial_{z} \delta(z / w)\right)+\mu \partial_{w}^{2} \delta(z / w) \\
& =-\partial_{w} b(w) \delta(z / w)+b(z) \partial_{w} \delta(z / w)+\mu \partial_{w}^{2} \delta(z / w) \\
& =b(w) \partial_{w} \delta(z / w)+\mu \partial_{w}^{2} \delta(z / w) .
\end{aligned}
$$

Corolário 4.2.17. Temos que

$$
\begin{gathered}
{\left[L_{-1}, a(z)\right]=\partial_{z} a(z),\left[L_{-1}, a^{*}(z)\right]=\partial_{z} a^{*}(z),\left[L_{-1}, b(z)\right]=\partial_{z} b(z)} \\
{\left[L_{0}, a(z)\right]=a(z)+z \partial_{z} a(z),\left[L_{0}, a^{*}(z)\right]=z \partial_{z} a^{*}(z),\left[L_{0}, b(z)\right]=b(z)+z \partial_{z} b(z) .}
\end{gathered}
$$

De fato, temos que

$$
\left[L_{-1}, a^{*}(z)\right]=-\sum_{k \in \mathbb{Z}}(k-1) a_{k-1}^{*} z^{-k}=-\sum_{s \in \mathbb{Z}} s a_{s}^{*} z^{-s-1}=\partial_{z} a^{*}(z) .
$$

Observação 4.2.20. $L(z)=a(z) \partial_{z} a^{*}(z)+\frac{1}{2}: b(z)^{2}:+\mu \partial_{z} b(z)$. 
Proposição 4.2.21. Temos as seguintes relações:

$$
\begin{aligned}
& {[\rho(f(z)), L(w)]=\rho(f(w)) \partial_{w} \delta(z / w)} \\
& {[\rho(h(z)), L(w)]=\rho(h(w)) \partial_{w} \delta(z / w)+\mu \partial_{w}^{2} \delta(z / w),} \\
& {[\rho(e(z)), L(w)]=\rho(e(w)) \partial_{w} \delta(z / w)-\mu \partial_{w}^{2}\left(a^{*}(w) \delta(z / w)\right) .}
\end{aligned}
$$

Demonstração. Temos

$$
\begin{aligned}
& {[\rho(f(z)), L(w)]=[a(z), L(w)]=a(w) \partial_{w} \delta(z / w)=\rho(f(w)) \partial_{w} \delta(z / w) . } \\
& {[\rho(h(z)), L(w)]=\left[2 a(z) a^{*}(z)+b(z), L(w)\right] } \\
&=-2 a(z)\left(\partial_{w} a^{*}(w)\right) \delta(z / w)+2 a(w) a^{*}(w) \partial_{z} \delta(z / w)+[b(z), L(w)] \\
&= 2 a(w)\left(-\partial_{w} a^{*}(w) \delta(z / w)+\partial_{w}\left(a^{*}(w) \delta(z / w)\right)+[b(z), L(w)]\right. \\
&= 2 a(w) a^{*}(w) \partial_{w} \delta(z / w)+b(w) \partial_{w} \delta(z / w)+\mu \partial_{w}^{2} \delta(z / w) \\
&= \rho(h(w)) \partial_{w} \delta(z / w)+\mu \partial_{w}^{2} \delta(z / w) . \\
& {[\rho(e(z)), L(w)]=-\left[a(z) a^{*}(z)^{2}+a^{*}(z) b(z)+\left(1-\gamma^{2}\right) \partial_{z} a^{*}(z), L(w)\right] } \\
&=-a(w) a^{*}(z)^{2} \partial_{w} \delta(z / w)+2 a(z) a^{*}(z) \partial_{w} a^{*}(w) \delta(z / w) \\
&-\left(b(w) \partial_{w} \delta(z / w)+\mu \partial_{w}^{2} \delta(z / w)\right) a^{*}(z)+b(z) \partial_{w} a^{*}(w) \delta(z / w) \\
&+\left(1-\gamma^{2}\right) \partial_{z}\left(\partial_{w} a^{*}(w) \delta(z / w)\right) \\
&=-a(w) a^{*}(w)^{2} \partial_{w} \delta(z / w)-b(w) a^{*}(w) \partial_{w} \delta(z / w) \\
&-\mu \partial_{w}^{2} \delta(z / w) a^{*}(z)+\left(1-\gamma^{2}\right) \partial_{w} a^{*}(w) \partial_{z} \delta(z / w) \\
&=-a(w) a^{*}(w)^{2} \partial_{w} \delta(z / w)-b(w) a^{*}(z) \partial_{w} \delta(z / w) \\
&-\mu\left(a^{*}(w) \partial_{w}^{2} \delta(z / w)+2 \partial_{w} a^{*}(w) \partial_{w} \delta(z / w)+\partial_{w}^{2} a^{*}(z) \delta(z / w)\right) \\
&-\left(1-\gamma^{2}\right) \partial_{w} a^{*}(w) \partial_{w} \delta(z / w) \\
&= \rho(e(w)) \partial_{w} \delta(z / w)-\mu\left(a^{*}(w) \partial_{w}^{2} \delta(z / w)+2 \partial_{w} a^{*}(w) \partial_{w} \delta(z / w)+\partial_{w}^{2} a^{*}(z) \delta(z / w)\right) \\
&= \rho(e(w)) \partial_{w} \delta(z / w)-\mu \partial_{w}^{2}\left(a^{*}(w) \delta(z / w)\right) .
\end{aligned}
$$

\section{Corolário 4.2.22.}

$$
\begin{gathered}
{\left[L_{-1}, \rho(f(z))\right]=\partial_{z} \rho(f(z)), \quad\left[L_{-1}, \rho(e(z))\right]=\partial_{z} \rho(e(z)),} \\
{\left[L_{-1}, \rho(h(z))\right]=\partial_{z} \rho(h(z)), \quad\left[L_{-1}, b(z)\right]=\partial_{z} b(z),} \\
{\left[L_{0}, \rho(f(z))\right]=z \partial_{z} \rho(f(z))+\rho(f(z)), \quad\left[L_{0}, \rho(e(z))\right]=z \partial_{z} \rho(e(z))+\rho(e(z)),} \\
{\left[L_{0}, \rho(h(z))\right]=z \partial_{z} \rho(h(z))+\rho(h(z)), \quad\left[L_{0}, b(z)\right]=z \partial_{z} b(z)+b(z),}
\end{gathered}
$$

como operadores em todo módulo de Verma imaginário.

\subsection{Operadores entrelaçantes}

\subsubsection{Módulos Topológicos}

Seguindo as definições dadas em [War93], página 22, temos 
Definição 4.3.1. Uma família $\mathcal{N}$ de subconjuntos de um conjunto $S$ é chamado filtro de $S$ se $S \in \mathcal{N}, \emptyset \notin \mathcal{N}$, a intersecção de dois elementos de $\mathcal{N}$ pertence a $\mathcal{N}$, e todo subconjunto de $S$ contendo um conjunto de $\mathcal{N}$ está em $\mathcal{N}$. Suponha que $\mathcal{B}$ é uma coleção de subconjuntos de um dado conjunto $S$ e considere o conjunto $\mathcal{N}=\{F \subseteq S \mid \exists B \in \mathcal{B}, B \subseteq F\}$. Se $\mathcal{N}$ é um filtro em $S$, então $\mathcal{B}$ é chamado um filtro base em $S$. Um sistema fundamental de vizinhanças de zero num espaço vetorial é qualquer filtro base que gere todas as vizinhanças de zero.

Teorema 4.3.2 ([War93], Teorema 3.5). Suponha que A é um anel. Se $\mathcal{N}$ é um filtro base de vizinhanças de $A$, então $\mathcal{N}$ satisfaz as seguintes condições:

(TRN1) Para todo $N \in \mathcal{N}$ existe $U \in \mathcal{N}$ tal que $U+U \subseteq N$.

(TRN2) $N \in \mathcal{N}$ implica que existe $U \in \mathcal{N}$ tal que $U \subseteq-N$.

(TRN3) Para todo $N \in \mathcal{N}$ existe $U \in \mathcal{N}$ tal que $U U \subseteq N$.

(TRN4) Para todo $N \in \mathcal{N}, b \in A$ existe $U \in \mathcal{N}$ tal que $b U \subseteq N$ e $U b \subseteq N$.

Se $\mathcal{N}$ é um filtro base em A satisfazendo (TRN1)-(TRN4), então existe um único anel topológico em A tal que $\mathcal{N}$ é um sistema fundamental de vizinhanças de zero.

Agora para definir módulos topológicos, precisamos de uma coleção de vizinhanças de zero $\mathfrak{n}=\{N\}$ tal que

(M1) Para cada $N \in \mathfrak{n}$ existe $U \in \mathfrak{n}$ tal que $U+U \subseteq \mathfrak{n}$.

(M2) Para cada $N \in \mathfrak{n}$ existe $U \in \mathfrak{n}$ tal que $U \subseteq-N$.

(M3) Para cada $N \in \mathfrak{n}$ existe uma vizinhança $T$ de zero em $A$ e $U \in \mathfrak{n}$ tal que $T U \subseteq N$.

(M4) Para cada $N \in \mathfrak{n}$ e cada $b \in E$ existe uma vizinhança $T$ de zero em $A$ tal que $T b \subseteq N$.

(M5) Para cada $N \in \mathfrak{n}$ e cada $\beta \in A$ existe $U \in \mathfrak{n}$ tal que $\beta U \subseteq \mathfrak{n}$.

Assim $\{N\}$ forma uma base para o módulo topológico $M$. Queremos encontrar um completamento de $M=U\left(\widehat{\mathfrak{g}}_{-}\right) \cdot 1 \subseteq V_{\lambda-m}$ e $W_{\lambda-m}$ tal que

$$
f_{m_{1}} \cdots f_{m_{k}}\left(\sum_{n \in \mathbb{N}} \sum_{\pi \in P_{n}} \alpha_{\pi} h_{\pi} 1\right) \in \widehat{V}_{\lambda-m}
$$

Sejam

$$
\begin{gathered}
U_{n}=\operatorname{span}\left\langle h_{n_{1}} \cdots h_{n_{k}} \mid n \leqslant \sum_{i=1}^{k} n_{i}\right\rangle \subseteq A, \\
\mathfrak{n}=\left\{U_{n} \mid n \in \mathbb{N}\right\} \text { uma topologia para } A, \\
\mathfrak{n}^{\prime}=\left\{U_{n} \cdot 1 \mid n \in \mathbb{N}\right\} \text { topologia para } M,
\end{gathered}
$$

e sejam

$$
\begin{gathered}
U_{n} \subseteq U\left(\widehat{\mathfrak{g}}_{-}\right)=A, M_{n}=U_{n} \cdot 1, M=U(\mathfrak{g}) \cdot 1, \\
\widehat{M}=\underbrace{\lim }_{n} M / M_{n}
\end{gathered}
$$

o completamento de $M$. Então temos

(M1) Se $N=M_{n} \in \mathfrak{n}$, então $U=M_{n}$ satisfaz $M_{n}+M_{n} \subseteq M_{n}$.

(M2) Se $N=M_{n} \in \mathfrak{n}$, então $U=M_{n}$ satisfaz $M=M_{n} \subseteq-N$.

(M3) Se $N=M_{n} \in \mathfrak{n}$, então para $T=U_{n}$ e $U=M_{n} \in \mathfrak{n}$ temos $T M=U_{n} U_{n} \cdot 1 \subseteq U_{2 n} \cdot 1 \subseteq$ 
$U_{n} \cdot 1=M_{n}$.

(M4) Para $N=M_{k} \in \mathfrak{n}, b=\sum_{n \in \mathbb{N}} \sum_{\pi \in P_{n}} \alpha_{\pi} h_{\pi} \cdot 1$, tome $T=U_{k}$ e então $U_{k} b \subseteq M_{k}$.

(M5) Para $N=M_{k} \in \mathfrak{n}, \beta=\sum_{n \in \mathbb{N}} \sum_{\pi \in P_{n}} \alpha_{\pi} h_{\pi} \in A$, tome $U=M_{k}$ e teremos $b M_{k} \subseteq M_{k}$.

Temos que ter certeza que $\widehat{M}$ pode ser visto como um $\widehat{\mathfrak{n}}_{-} \oplus \widehat{\mathfrak{h}}$-módulo. Precisamos verificar que a multiplicação à esquerda por $h_{n}, n \in \mathbb{N}$, é contínua: $h_{n} M_{m} \subseteq M_{n+m}$. Logo $h_{n}$ se estende a uma aplicação $h_{n}: \widehat{M} \longrightarrow \widehat{M}$.

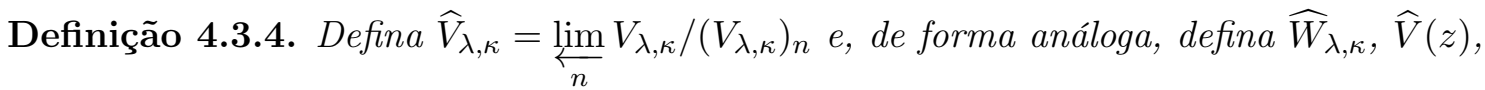
etc.

Definição 4.3.5. Seja $\mathfrak{F}_{m}$ o $\mathfrak{s l}(2, \mathbb{C})$-módulo de dimensão finita com peso máximo $m \in \mathbb{N}$ e vetor de peso máximo $u^{m}$.

Observe que as definições de $\mathfrak{F}_{m}(z)$ e $V_{\lambda-m, \kappa} \widehat{\otimes} \mathfrak{F}_{m}(z)$ são similares às definições dadas em (4.1.15) e (4.1.17). Seja $u_{k}^{m}=u^{m} \otimes z^{k} \in \mathfrak{F}_{m}(z)$, para $k \in \mathbb{Z}$.

Definição 4.3.6. Defina $P_{n}=\left\{\left(-n_{1},-n_{2}, \cdots,-n_{p}\right) \mid n_{1}+\cdots+n_{p}=n\right\}$ e, para $\pi=$ $\left(-n_{1}, \cdots,-n_{p}\right) \in P_{n}$ fixado, defina $h_{\pi}=h_{n_{1}} \cdots h_{n_{p}}$.

Proposição 4.3.7. Suponha $\kappa \neq 0$ e fixe $\beta_{(1)} \in \mathbb{C}$. Sejam $m \in \mathbb{N}, V_{\lambda-m, \kappa}$ o módulo de Verma imaginário para $\widehat{\mathfrak{g}}$ e $v_{\lambda-m, \kappa} \in V_{\lambda-m, \kappa}$ um vetor de peso máximo imaginário com peso máximo $\lambda-m$. Para $\left(n_{1}, n_{2}, \ldots, n_{r}\right)=\left(n_{1}, n_{2}, \ldots, n_{r}, 0,0, \ldots\right)$, com $n_{i} \in \mathbb{N}$, defina

$$
\beta_{\left(n_{1}, \ldots, n_{r}\right)}=\frac{m^{n_{r}+\cdots+n_{1}-1}}{(-\kappa)^{n_{r}+\cdots+n_{1}-1}\left(r^{n_{r}} \cdots \cdots 2^{n_{2}}\right) \cdot n_{r} ! \ldots n_{1} !} \cdot \beta_{(1)}
$$

Para uma partição

$$
\pi=(\underbrace{-1,-1, \ldots,-1}_{n_{1}}, \underbrace{-2,-2, \ldots,-2}_{n_{2}}, \ldots, \underbrace{-r,-r, \ldots,-r}_{n_{r}})
$$

de $-n\left(\log o n=\sum_{l=1}^{r} \ln _{l}\right)$, defina

$$
\alpha_{(\underbrace{-1,-1, \ldots,-1}_{n_{1}}} \underbrace{-2,-2, \ldots,-2}_{n_{2}}, \ldots, \underbrace{-r,-r, \ldots,-r}_{n_{r}})=\beta_{\left(n_{1}, n_{2}, \ldots, n_{r}\right)}
$$

$e$

$$
h_{\pi}=h_{-1}^{n_{1}} h_{-2}^{n_{2}} \cdots h_{-r}^{n_{r}}
$$

O vetor

$$
v_{\lambda, \kappa}^{\sharp}=\sum_{n \in \mathbb{N}} \sum_{\pi \in P_{n}} \alpha_{\pi} h_{\pi} v_{\lambda-m, \kappa} \otimes u_{n}^{m} \in V_{\lambda-m, \kappa} \widehat{\otimes} \mathfrak{F}_{m}(z)
$$

é um vetor de peso máximo imaginário $\lambda$. Então existe um $\widehat{\mathfrak{g}}$-módulo homomorfismo nãonulo $\Phi^{V}(z): V_{\lambda, \kappa} \rightarrow V_{\lambda-m, \kappa} \widehat{\otimes} \mathfrak{F}_{m}(z)$ tal que $\Phi^{V}(z)\left(v_{\lambda, \kappa}\right)=v_{\lambda, \kappa}^{\sharp}$. 
Demonstração. Faremos um esboço da demonstração para o caso da ação de $h_{1}$.

$$
\begin{aligned}
& h_{1}\left(\alpha_{0} v_{\lambda-m, \kappa} \otimes u_{0}^{m}+\alpha_{(-1)} h_{-1} v_{\lambda-m, \kappa} \otimes u_{1}^{m}+\left(\alpha_{(-1,-1)} h_{-1}^{2}+\alpha_{(-2)} h_{-2}\right) v_{\lambda-m, \kappa} \otimes u_{2}^{m}\right. \\
& \quad+\left(\alpha_{(-1,-1,-1)} h_{-1}^{3}+\alpha_{(-2,-1)} h_{-1} h_{-2}+\alpha_{(-3)} h_{-3}\right) v_{\lambda-m, \kappa} \otimes u_{3}^{m} \\
& \quad+\left(\alpha_{(-1,-1,-1,-1)} h_{-1}^{4}+\alpha_{(-2,-1,-1)} h_{-1}^{2} h_{-2}+\alpha_{(-2,-2)} h_{-2}^{2}\right. \\
& \left.\left.\quad+\alpha_{(-3,-1)} h_{-1} h_{-3}+\alpha_{(-4)} h_{-4}\right) v_{\lambda-m, \kappa} \otimes u_{4}^{m}+\cdots\right) \\
& =0+m \alpha_{0} v_{\lambda-m, \kappa} \otimes u_{1}^{m}+\alpha_{(-1)} \kappa v_{\lambda-m, \kappa} \otimes u_{1}^{m}+m \alpha_{(-1)} h_{-1} v_{\lambda-m, \kappa} \otimes u_{2}^{m} \\
& \quad+2 \alpha_{(-1,-1)} \kappa h_{-1} v_{\lambda-m, \kappa} \otimes u_{2}^{m}+m \alpha_{(-1,-1)} h_{-1}^{2} v_{\lambda-m, \kappa} \otimes u_{3}^{m}+m \alpha_{(-2)} h_{-2} v_{\lambda-m, \kappa} \otimes u_{3}^{m} \\
& \quad+3 \alpha_{(-1,-1,-1)} \kappa h_{-1}^{2} v_{\lambda-m, \kappa} \otimes u_{3}^{m}+m \alpha_{(-1,-1,-1)} h_{-1}^{3} v_{\lambda-m, \kappa} \otimes u_{4}^{m} \\
& \quad+\alpha_{(-2,-1)} \kappa h_{-2} v_{\lambda-m, \kappa} \otimes u_{3}^{m}+m \alpha_{(-2,-1)} h_{-1} h_{-2} v_{\lambda-m, \kappa} \otimes u_{4}^{m}+m \alpha_{(-3)} h_{-3} v_{\lambda-m, \kappa} \otimes u_{4}^{m}+ \\
& \quad+\kappa\left(4 \alpha_{(-1,-1,-1,-1)} h_{-1}^{3}+2 \alpha_{(-2,-1,-1)} h_{-1} h_{-2}+\alpha_{(-3,-1)} h_{-3}\right) v_{\lambda-m, \kappa} \otimes u_{4}^{m}+\cdots
\end{aligned}
$$

Para $1 \leqslant k \leqslant r$, temos

$$
\begin{aligned}
h_{k}\left(\beta_{\left(n_{1}, n_{2}, \ldots, n_{r}\right)}\right. & \left.h_{-1}^{n_{1}} \ldots h_{-k}^{n_{k}} \ldots h_{-r}^{n_{r}} v_{\lambda-m, \kappa} \otimes u_{n}^{m}\right) \\
= & k \cdot n_{k} \cdot \kappa \cdot \beta_{\left(n_{1}, \ldots, n_{k}, \ldots, n_{r}\right)} h_{-1}^{n_{1}} \ldots h_{-k}^{n_{k}-1} \ldots h_{-r}^{n_{r}} v_{\lambda-m, \kappa} \otimes u_{n}^{m} \\
& +m \beta_{\left(n_{1}, \ldots, n_{k}, \ldots, n_{r}\right)} h_{-1}^{n_{1}} \ldots h_{-k}^{n_{k}} \ldots h_{-r}^{n_{r}} v_{\lambda-m, \kappa} \otimes u_{n+k}^{m} .
\end{aligned}
$$

Por esta razão temos, no caso geral, que

$$
m \beta_{\left(n_{1}, \ldots, n_{k}-1, \ldots, n_{r}\right)}+k . n_{k} . \kappa \beta_{\left(n_{1}, \ldots, n_{k}, \ldots, n_{r}\right)}=0 .
$$

Neste caso, quando aplicamos $h_{k}$, o coeficiente de $h_{-1}^{n_{1}} \ldots h_{-k}^{n_{k}-1} \ldots h_{-r}^{n_{r}} v_{\lambda-m, \kappa} \otimes u_{n}^{m}$ (onde $\left.n=\sum_{i=1}^{r} i n_{i}\right)$ é $m \beta_{\left(n_{1}, \ldots, n_{k}-1, \ldots, n_{r}\right)}+k \cdot n_{k} \cdot \kappa \beta_{\left(n_{1}, \ldots, n_{k}, \ldots, n_{r}\right)}$ e este será zero por definição. Então fixemos $\beta_{(1)}$ e encontremos todas as outras condições observando que

$$
\begin{gathered}
m \beta_{(1)}+l . \kappa \beta_{(1,0, \ldots, 0,1)}=0 \quad \Longrightarrow \quad \beta_{(1,0, \ldots, 0,1)}=\frac{-m}{l \cdot \kappa} \beta_{(1)}, \\
m \beta_{(0, \ldots, 0,1)}+1 . \kappa \beta_{(1,0, \ldots, 0,1)}=0 \quad \Longrightarrow \quad \beta_{(0,0, \ldots, 0,1)}=\frac{1}{l} \beta_{(1)},
\end{gathered}
$$

onde 1 aparece na (última) l-entrada de $(1,0, \ldots, 0,1)$ e $(0, \ldots, 0,1)$. Similarmente, aplicando $h_{k}$ repetidamente, teremos

$$
m^{n_{k}} \beta_{\left(n_{1}, \ldots, n_{k-1}, 0, n_{k+1}, \ldots, n_{r}\right)}=(-1)^{n_{k}} \cdot k^{n_{k}} \cdot \kappa^{n_{k}} \cdot n_{k} ! \beta_{\left(n_{1}, \ldots, n_{k}, \ldots, n_{r}\right)} .
$$

Sem perda de generalidade, podemos assumir que $n_{1} \neq 0$ e obter

$$
\beta_{(1)}=\frac{(-\kappa)^{n_{r}+\cdots+n_{1}-1} \cdot\left(r^{n_{r}} \ldots 2^{n_{2}}\right) \cdot n_{r} ! \ldots n_{1} !}{m^{n_{r}+\cdots+n_{1}-1}} \cdot \beta_{\left(n_{1}, \ldots, n_{r}\right)} .
$$


Sob a hipótese $\kappa \neq 0$,

$$
\beta_{\left(n_{1}, \ldots, n_{r}\right)}=\frac{m^{n_{r}+\cdots+n_{1}-1}}{(-\kappa)^{n_{r}+\cdots+n_{1}-1}\left(r^{n_{r}} \ldots 2^{n_{2}}\right) \cdot n_{r} ! \ldots n_{1} !} \cdot \beta_{(1)}
$$

está bem-definida e força $v_{\lambda, \kappa}^{\sharp}$ a ser aniquilada por $h_{k}$, para todo $k \geq 1$. Assim temos

$$
\begin{aligned}
h_{m}\left(v_{\lambda, \kappa}^{\sharp}\right) & =0, \\
e_{m}\left(v_{\lambda, \kappa}^{\sharp}\right) & =\sum_{n \in \mathbb{N}^{*}} \sum_{\pi \in P_{n}} \alpha_{\pi}\left(e_{m} h_{\pi} v_{\lambda-m, \kappa}\right) \otimes u_{n}^{m}+\sum_{n \in \mathbb{N}^{*}} \sum_{\pi \in P_{n}} \alpha_{\pi} h_{\pi} v_{\lambda-m, \kappa} \otimes e_{m} u_{n}^{m} \\
& =0,
\end{aligned}
$$

para todo $m>0$ (já que $e_{m} h_{k}=-2 e_{m+k}+h_{k} e_{m}, \forall k$ ). Então $v_{\lambda, \kappa}^{\sharp}$ é um vetor de peso máximo imaginário de peso $\lambda+\kappa \Lambda_{0}$. Pela propriedade universal das aplicações dos módulos de Verma imaginários, existe um homomorfismo de $\widehat{\mathfrak{g}}$-módulo $\varphi: V_{\lambda, \kappa} \longrightarrow V_{\lambda-m, \kappa} \widehat{\otimes} \mathfrak{F}_{m}(z)$, para $\kappa \neq 0$, mandando $v_{\lambda, \kappa}$ para $v_{\lambda, \kappa}^{\sharp}$.

\section{Corolário 4.3.9.}

$$
\left((z h(z))_{-} \otimes 1\right) \widehat{\Phi}^{V}(z)\left(v_{\lambda, \kappa}\right)=-m \widehat{\Phi}^{V}(z)\left(v_{\lambda, \kappa}\right) .
$$

Demonstração. Por (4.3.8), temos

$$
\begin{aligned}
\left(h_{k} \otimes 1\right)\left(\beta_{\left(n_{1}, n_{2}, \ldots, n_{r}\right)}\right. & \left.h_{-1}^{n_{1}} \ldots h_{-k}^{n_{k}} \ldots h_{-r}^{n_{r}} v_{\lambda-m, \kappa} \otimes u_{n}^{m}\right) \\
& =k \cdot n_{k} \cdot \kappa \cdot \beta_{\left(n_{1}, \ldots, n_{k}, \ldots, n_{r}\right)} h_{-1}^{n_{1}} \ldots h_{-k}^{n_{k}-1} \ldots h_{-r}^{n_{r}} v_{\lambda-m, \kappa} \otimes u_{n}^{m} \\
& =-m \beta_{\left(n_{1}, \ldots, n_{k}-1, \ldots, n_{r}\right)} h_{-1}^{n_{1}} \ldots h_{-k}^{n_{k}-1} \ldots h_{-r}^{n_{r}} v_{\lambda-m, \kappa} \otimes u_{n}^{m}
\end{aligned}
$$

e então

$$
\begin{aligned}
\left((z h(z))_{-} \otimes 1\right) \widehat{\Phi}^{V}(z)\left(v_{\lambda, \kappa}\right) & =\sum_{k>0} \sum_{n \in \mathbb{N}} \sum_{\pi \in P_{n}} \alpha_{\pi}\left[h_{k}, h_{\pi}\right] v_{\lambda-m, \kappa} \otimes z^{-\Delta} u_{n}^{m} z^{-k} \\
& =-m \sum_{k>0} \sum_{n \in \mathbb{N}} \sum_{\pi \in P_{n}} \alpha_{\pi} h_{\pi} v_{\lambda-m, \kappa} \otimes z^{-\Delta-k} u_{n+k}^{m} \\
& =-m z^{-\Delta} \sum_{n \in \mathbb{N}} \sum_{\pi \in P_{n}} \alpha_{\pi} h_{\pi} v_{\lambda-m, \kappa} \otimes u_{n}^{m} \\
& =-m \widehat{\Phi}^{V}(z)\left(v_{\lambda, \kappa}\right) .
\end{aligned}
$$

Em particular observe a propriedade entrelaçante de $\Phi^{V}(z)$ : Se denotarmos por $*$ a ação do produto tensorial, então

$$
\Phi^{V}(z) x_{n}=x_{n} * \Phi(z)=\left(x_{n} \otimes 1+z^{n}(1 \otimes x)\right) \Phi^{V}(z)
$$

para $x \in \mathfrak{s l}(2, \mathbb{C})$. 


\subsubsection{Forma operadora das equações de Knizhnik-Zamolodchikov}

Defina $\Phi^{W}(z): W_{\lambda, \kappa} \rightarrow W_{\lambda-m, \kappa} \widehat{\otimes} \mathfrak{F}_{m}(z)$ de forma a satisfazer o seguinte diagrama

$$
\begin{aligned}
& W_{\lambda, \kappa} \stackrel{\Phi^{W}(z)}{\longrightarrow} W_{\lambda-m, \kappa} \widehat{\otimes} \mathfrak{F}_{m}(z) \\
& \Psi_{\lambda, \kappa}^{-1} \downarrow \quad \Psi_{\lambda-m, \kappa} \otimes 1 \uparrow \\
& V_{\lambda, \kappa} \stackrel{\Phi^{V}(z)}{\longrightarrow} V_{\lambda-m, \kappa} \widehat{\otimes} \mathfrak{F}_{m}(z)
\end{aligned}
$$

onde $\Psi_{\lambda, \kappa}: V_{\lambda, \kappa} \rightarrow W_{\lambda, \kappa}$ é o isomorfismo canônico dado no Teorema $4.1 .22 \mathrm{e}$

$$
\left(\Psi_{\lambda-m, \kappa} \otimes 1\right)\left(v_{1} \otimes v_{2}\right)=\Psi_{\lambda-m, \kappa}\left(v_{1}\right) \otimes v_{2} .
$$

Então

$$
\Phi^{W}(z)=\left(\Psi_{\lambda-m, \kappa} \otimes 1\right) \circ \Phi^{V}(z) \circ \Psi_{\lambda, \kappa}^{-1} .
$$

Considere $z$ como uma variável formal $\mathrm{e}$

$$
\mathfrak{F}_{m} \otimes z^{-\Delta} \mathbb{C}\left[z, z^{-1}\right]=z^{-\Delta} \mathfrak{F}_{m}\left[z_{1}, z_{1}^{-1}\right]
$$

que é uma representação de dimensão infinita de $\widehat{\mathfrak{g}}$ com base $v \otimes z^{n-\Delta}, \quad n \in \mathbb{Z}, \quad v \in \mathfrak{F}_{m}$.

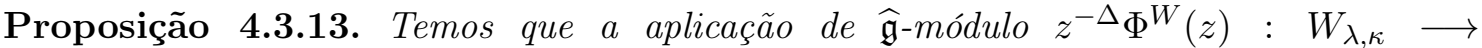
$W_{\lambda-m, \kappa} \widehat{\otimes} z^{-\Delta} \mathfrak{F}_{m}\left[z, z^{-1}\right]$ é um homomorfismo de $\widetilde{\mathfrak{g}}-$ módulo (aqui d age como $z \frac{\partial}{\partial z}$ no segundo fator)

$$
z^{-\Delta} \Phi^{W}(z)\left(w_{\lambda, \kappa}\right)=\sum_{n \in \mathbb{N}^{*}} \sum_{\pi \in P_{n}} \alpha_{\pi} b_{\pi} w_{\lambda-m, \kappa} \otimes z^{-\Delta} u_{n}^{m}
$$

se, e somente se,

$$
\Delta=\Delta(\lambda)-\Delta(\lambda-m) .
$$

Demonstração. Por definição, temos que $d \cdot w_{\lambda, \kappa}=-\Delta(\lambda) \cdot w_{\lambda, \kappa}$, para $w_{\lambda, \kappa} \in W_{\lambda, \kappa}$ (ver (4.1.13)). Agora $z^{-\Delta} \Phi^{W}(z)$ é $\widetilde{\mathfrak{g}}$-entrelaçante se, e somente se, satisfaz

$$
z^{-\Delta} \Phi^{W}(z) d-(d \otimes 1) z^{-\Delta} \Phi^{W}(z)=\left(1 \otimes z \frac{\partial}{\partial z}\right) z^{-\Delta} \Phi^{W}(z) .
$$

Apliquemos ambos os lados desta igualdade ao vetor de peso máximo imaginário $w_{\lambda, \kappa}$ e teremos que esta é verdadeira se, e somente se, a condição (4.3.14) for satisfeita. Então como $W_{\lambda, \kappa}$ é gerada por este vetor, os dois lados valerão para todo o $W_{\lambda, \kappa}$ se, e somente se, (4.3.14) for satisfeita. O lado esquerdo de (4.3.15) aplicado à $w_{\lambda, \kappa}$ nos dá

$$
\begin{aligned}
\left(z^{-\Delta} \Phi^{W}(z) d-\right. & \left.(d \otimes 1) z^{-\Delta} \Phi^{W}(z)\right)\left(w_{\lambda, \kappa}\right) \\
= & -\Delta(\lambda) \sum_{n \in \mathbb{N}^{*}} \sum_{\pi \in P_{n}} \alpha_{\pi} b_{\pi} w_{\lambda-m, \kappa} \otimes u_{n}^{m} z^{-\Delta} \\
& -\sum_{n \in \mathbb{N}^{*}} \sum_{\pi \in P_{n}} \alpha_{\pi}(-n-\Delta(\lambda-m)) b_{\pi} w_{\lambda-m, \kappa} \otimes u_{n}^{m} z^{-\Delta} \\
= & -(\Delta(\lambda)-\Delta(\lambda-m)) \sum_{n \in \mathbb{N}^{*}} \sum_{\pi \in P_{n}} \alpha_{\pi} b_{\pi} w_{\lambda-m, \kappa} \otimes u_{n}^{m} z^{-\Delta} \\
& +\sum_{n \in \mathbb{N}^{*}} \sum_{\pi \in P_{n}} n \alpha_{\pi} b_{\pi} w_{\lambda-m, \kappa} \otimes u_{n}^{m} z^{-\Delta}
\end{aligned}
$$


enquanto que o lado direito nos dá

$$
\begin{aligned}
\left(1 \otimes z \frac{\partial}{\partial z}\right) & z^{-\Delta} \Phi^{W}(z)\left(w_{\lambda, \kappa}\right)=\sum_{\pi \in P_{n}} \alpha_{\pi} b_{\pi} w_{\lambda-m, \kappa} \otimes z \frac{\partial}{\partial z} u_{n}^{m} z^{-\Delta} \\
& =-\Delta \sum_{\pi \in P_{n}} \alpha_{\pi} b_{\pi} w_{\lambda-m, \kappa} \otimes u_{n}^{m} z^{-\Delta}+\sum_{\pi \in P_{n}} n \alpha_{\pi} b_{\pi} w_{\lambda-m, \kappa} \otimes u_{n}^{m} z^{-\Delta} .
\end{aligned}
$$

Para completar a demonstração, basta usar (4.3.12) e ver que $\Psi_{\lambda, \kappa}$ é um homomorfismo de $\widetilde{\mathfrak{g}}$-módulo.

Para $x \in \mathfrak{F}_{m}^{*}$, defina $\Phi_{x}^{V}(z): V_{\lambda, \kappa} \longrightarrow \widehat{V}_{\lambda-m, \kappa}$ por

$$
\Phi_{x}^{V}(z)(w)=x\left(\Phi^{V}(z)(w)\right) .
$$

Por exemplo,

$$
\Phi_{x}^{V}(z)\left(v_{\lambda, \kappa}\right)=\sum_{n \in \mathbb{N}^{*}} \sum_{\pi \in P_{n}} \alpha_{\pi} h_{\pi} v_{\lambda-m, \kappa} x\left(u_{n}^{m}\right)
$$

onde $x u_{n}^{m}=\left(x u^{m}\right) z^{n}$. Analogamente, defina $\Phi_{x}^{W}(z): W_{\lambda, \kappa} \rightarrow \widehat{W}_{\lambda-m, \kappa}$. A propriedade entrelaçante (4.3.15), para $x=f$, nos dá

$$
\begin{aligned}
\Phi_{x}^{W}(z) a_{m} & =\Phi_{x}^{W}(z) f_{m}=f_{m} * \Phi_{x}^{W}(z)=\left(f_{m} \otimes 1\right) \Phi_{x}^{W}(z)+z^{m}(1 \otimes f) \Phi_{x}^{V}(z) \\
& =\left(a_{m} \otimes 1\right) \Phi_{x}^{W}(z)-z^{m} \Phi_{f x}^{W}(z) .
\end{aligned}
$$

onde $f x$ denota a ação de $f$ em um elemento do espaço dual $\mathfrak{F}_{m}^{*}$. Então

$$
\left[a_{m}, \Phi_{x}^{W}(z)\right]=z^{m} \Phi_{f x}^{W}(z)
$$

Cálculos similares mostram que

$$
\left[b_{m}, \Phi_{x}^{W}(z)\right]=z^{m} \Phi_{h x}^{W}(z) .
$$

As equações (4.3.18) e (4.3.19) mostram que

$$
\begin{aligned}
& {\left[b(\zeta)_{+}, \Phi_{x}^{W}(z)\right]=\frac{1}{z-\zeta} \Phi_{h x}^{W}(z),} \\
& {\left[b(\zeta)_{-}, \Phi_{x}^{W}(z)\right]=\frac{1}{\zeta-z} \Phi_{h x}^{W}(z),} \\
& {\left[a(\zeta), \Phi_{x}^{W}(z)\right]=\Phi_{f x}^{W}(z) \delta(\zeta / z) .}
\end{aligned}
$$

Para $x \in \mathfrak{F}_{m}^{*}$ e $\Delta=\Delta(\lambda)-\Delta(\lambda-m)$, seja

$$
\widetilde{\Phi}_{x}^{W}(z)=z^{-\Delta} \Phi_{x}^{W}(z) .
$$


Teorema 4.3.24 (Forma operadora das equações de Knizhnik-Zamolodchikov). Para $x \in \mathfrak{F}_{m}^{*}$ de peso $\alpha \in \mathbb{C}$, seja

$$
\Delta(\mu, \alpha)=\frac{\alpha(\alpha-2 \mu)}{4} e \widehat{\Phi}_{x}^{W}(z)=z^{-\Delta(\mu, \alpha)} \widetilde{\Phi}_{x}^{W}(z)
$$

onde $\mu$ foi fixado anteriormente (ver Definição 4.2.6).

O operador $\widehat{\Phi}_{x}^{W}(z): W_{\lambda, \kappa} \longrightarrow W_{\lambda-m, \kappa} \widehat{\otimes}_{m}\left[z, z^{-1}\right] \cdot z^{-\Delta}$ satisfaz a equação diferencial

$$
\frac{d}{d z} \widehat{\Phi}_{x}^{W}(z)=\widehat{\Phi}_{f x}^{W}(z) \partial_{z} a^{*}(z)+\frac{\alpha}{2}: b(z) \widehat{\Phi}_{x}^{W}(z): .
$$

Demonstração.

$$
\begin{aligned}
\widehat{\Phi}_{x}^{W}(z) & \circ a_{n}^{*}\left(a_{n_{1}} \cdots a_{n_{k}} b_{-m_{1}} \cdots b_{-m_{l}} \cdot w_{\lambda, \kappa}\right) \\
& \left.=-\sum_{i=1}^{k} \delta_{n+n_{i}, 0} \widehat{\Phi}_{x}^{W}(z)\left(a_{n_{1}} \cdots a_{n_{i-1}} a_{n_{i+1}} \cdots a_{n_{k}} b_{-m_{1}} \cdots b_{-m_{l}} \cdot w_{\lambda, \kappa}\right)\right)
\end{aligned}
$$

enquanto que

$$
\begin{aligned}
a_{n}^{*} \circ \widehat{\Phi}^{W}(z)\left(a_{n_{1}} \cdots a_{n_{k}} b_{-m_{1}} \cdots b_{-m_{l}} \cdot w_{\lambda, \kappa}\right) & \\
= & a_{n}^{*} \circ\left(a_{n_{1}} * \cdots * a_{n_{k}} * b_{-m_{1}} * \cdots * b_{-m_{l}} * \widehat{\Phi}^{W}(z)\left(w_{\lambda, \kappa}\right)\right) \\
= & a_{n}^{*}\left(\left(a_{n_{1}} \otimes 1+z^{n_{1}} \otimes f\right) \cdots\left(a_{n_{k}} \otimes 1+z^{n_{k}} \otimes f\right)\right. \\
& \left.\times\left(b_{-m_{1}} \otimes 1+z^{-m_{1}} \otimes h\right) \cdots\left(b_{-m_{l}} \otimes 1+z^{-m_{l}} \otimes h\right)\right) \widehat{\Phi}^{W}(z)\left(w_{\lambda, \kappa}\right) \\
= & -\sum_{i=1}^{k} \delta_{n+n_{i}, 0}\left(a_{n_{1}} \otimes 1+z^{n_{1}} \otimes f\right) \cdots\left(a_{n_{i-1}} \otimes 1+z^{n_{i-1}} \otimes f\right) \\
& \cdot\left(a_{n_{i+1}} \otimes 1+z^{n_{i+1}} \otimes f\right) \cdots\left(a_{n_{k}} \otimes 1+z^{n_{k}} \otimes f\right) \\
& \cdot\left(b_{-m_{1}} \otimes 1+z^{-m_{1}} \otimes h\right) \cdots\left(b_{-m_{l}} \otimes 1+z^{-m_{l}} \otimes h\right) \cdot \widehat{\Phi}^{W}(z)\left(w_{\lambda, \kappa}\right) \\
& \left.+a_{n_{1}} * \cdots * a_{n_{k}} * b_{-m_{1}} * \cdots * b_{-m_{l}} *\left(a_{n}^{*} \otimes 1\right) \widehat{\Phi}^{W}(z)\left(w_{\lambda, \kappa}\right)\right) \\
= & -\sum_{i=1}^{k} \delta_{n+n_{i}, 0} \widehat{\Phi}^{W}(z)\left(a_{n_{1}} \cdots a_{n_{i-1}} a_{n_{i+1}} \cdots a_{n_{k}} b_{-m_{1}} \cdots b_{-m_{l}} \cdot w_{\lambda, \kappa}\right) \\
& \left.+a_{n_{1}} * \cdots * a_{n_{k}} * b_{-m_{1}} * \cdots * b_{-m_{l}} *\left(a_{n}^{*} \otimes 1\right) \widehat{\Phi}^{W}(z)\left(w_{\lambda, \kappa}\right)\right) .
\end{aligned}
$$

Por outro lado,

$$
a_{n}^{*} \widehat{\Phi}^{W}(z)\left(w_{\lambda, \kappa}\right)=\sum_{n \in \mathbb{N}} \sum_{\pi \in P_{n}} \alpha_{\pi} a_{n}^{*} b_{\pi} w_{\lambda-m, \kappa} \otimes z^{-\Delta} u_{n}^{m}=0
$$

e assim, 


$$
\begin{aligned}
\left(a_{n}^{*} \otimes 1\right) \circ \widehat{\Phi}_{x}^{W}(z)\left(a_{n_{1}} \cdots a_{n_{k}} b_{-m_{1}} \cdots b_{-m_{l}} \cdot w_{\lambda, \kappa}\right) & \\
& =(1 \otimes x) \circ\left(a_{n}^{*} \otimes 1\right) \circ \widehat{\Phi}^{W}(z)\left(a_{n_{1}} \cdots a_{n_{k}} b_{-m_{1}} \cdots b_{-m_{l}} \cdot w_{\lambda, \kappa}\right) \\
& =-\sum_{i=1}^{k} \delta_{n+n_{i}, 0}(1 \otimes x) \widehat{\Phi}^{W}(z)\left(a_{n_{1}} \cdots a_{n_{i-1}} a_{n_{i+1}} \cdots a_{n_{k}} b_{-m_{1}} \cdots b_{-m_{l}} \cdot w_{\lambda, \kappa}\right) \\
& =-\sum_{i=1}^{k} \delta_{n+n_{i}, 0} \widehat{\Phi}_{x}^{W}(z)\left(a_{n_{1}} \cdots a_{n_{i-1}} a_{n_{i+1}} \cdots a_{n_{k}} b_{-m_{1}} \cdots b_{-m_{l}} \cdot w_{\lambda, \kappa}\right) .
\end{aligned}
$$

$\operatorname{Logo}\left[a_{n}^{*}, \widehat{\Phi}_{x}^{W}(z)\right]=0$. Temos

$$
z \frac{d}{d z} \widehat{\Phi}_{x}^{W}(z)=-\left[d, \widetilde{\Phi}_{x}^{W}(z)\right]-\Delta(\mu, \alpha) \widehat{\Phi}_{x}^{W}(z) .
$$

Substituindo $d$ por $-L_{0}$ e usando (4.2.7), temos

$$
\begin{aligned}
z \frac{d}{d z} \widehat{\Phi}_{x}^{W}(z)= & {\left[L_{0}, \widehat{\Phi}_{x}^{W}(z)\right]-\Delta(\mu, \alpha) \widehat{\Phi}_{x}^{W}(z) } \\
= & {\left[\sum_{n \in \mathbb{Z}} n a_{n} a_{-n}^{*}+\frac{1}{4}\left(\sum_{n \in \mathbb{Z}}: b_{n} b_{-n}:\right)-\frac{\mu}{2} b_{0}, \widehat{\Phi}_{x}^{W}(z)\right]-\Delta(\mu, \alpha) \widehat{\Phi}_{x}^{W}(z) } \\
= & \sum_{n \in \mathbb{Z}} n\left[a_{n}, \widehat{\Phi}_{x}^{W}(z)\right] a_{-n}^{*}-\Delta(\mu, \alpha) \widehat{\Phi}_{x}^{W}(z) \\
& +\left[\frac{1}{4}\left(\sum_{n>0} b_{-n} b_{n}\right)+\frac{1}{4}\left(\sum_{n<0} b_{n} b_{-n}\right)+\frac{1}{4} b_{0}^{2}-\frac{\mu}{2} b_{0}, \widehat{\Phi}_{x}^{W}(z)\right] \\
= & \widehat{\Phi}_{f x}^{W}(z) \sum_{n \in \mathbb{Z}} n a_{-n}^{*} z^{n}-\Delta(\mu, \alpha) \widehat{\Phi}_{x}^{W}(z) \\
& +\frac{1}{2}\left(\sum_{n>0}\left[b_{-n}, \widehat{\Phi}_{x}^{W}(z)\right] b_{n}\right)+\frac{1}{2}\left(\sum_{n>0} b_{-n}\left[b_{n}, \widehat{\Phi}_{x}^{W}(z)\right]\right) \\
& +\frac{1}{4}\left(\left[b_{0}, \widehat{\Phi}_{x}^{W}(z)\right] b_{0}+b_{0}\left[b_{0}, \widehat{\Phi}_{x}^{W}(z)\right]\right)-\frac{\mu}{2}\left[b_{0}, \widehat{\Phi}_{x}^{W}(z)\right] \\
= & \widehat{\Phi}_{f x}^{W}(z) z \partial_{z} a^{*}(z)-\Delta(\mu, \alpha) \widehat{\Phi}_{x}^{W}\left(z+\frac{1}{2}\left(\sum_{n>0} \widehat{\Phi}_{h x}^{W}(z) b_{n} z^{-n}\right)\right. \\
& +\frac{1}{2}\left(\sum_{n>0} b_{-n} \widehat{\Phi}_{h x}^{W}(z) z^{n}\right)+\frac{1}{4}\left(\widehat{\Phi}_{h x}^{W}(z) b_{0}+b_{0} \widehat{\Phi}_{h x}^{W}(z)\right)-\frac{\mu}{2} \widehat{\Phi}_{h x}^{W}(z) \\
= & \widehat{\Phi}_{f x}^{W}(z) z \partial_{z} a^{*}(z)-\Delta(\mu, \alpha) \widehat{\Phi}_{x}^{W}(z)+\frac{z}{2}: b(z) \widehat{\Phi}_{h x}^{W}(z): \\
& +\frac{1}{4}\left(-\widehat{\Phi}_{h x}^{W}(z) b_{0}+b_{0} \widehat{\Phi}_{h x}^{W}(z)\right)-\frac{\mu}{2} \widehat{\Phi}_{h x}^{W}(z) \\
= & \widehat{\Phi}_{f x}^{W}(z) z \partial_{z} a^{*}(z)+\frac{z}{2}: b(z) \widehat{\Phi}_{h x}^{W}(z):+\frac{1}{4} \widehat{\Phi}_{h^{2} x}^{W}(z)-\frac{\mu}{2} \widehat{\Phi}_{h x}^{W}(z)-\Delta(\mu, \alpha) \widehat{\Phi}_{x}^{W}(z) \\
= & \widehat{\Phi}_{f x}^{W}(z) z \partial_{z} a^{*}(z)+\frac{\alpha z}{2}: b(z) \widehat{\Phi}_{x}^{W}(z): . \\
& \\
& \\
&
\end{aligned}
$$


Teorema 4.3.26. Seguindo as notações do Teorema anterior, temos

$$
\left[L_{m}, \widehat{\Phi}_{x}^{W}(z)\right]=\widehat{\Phi}_{f x}^{W}(z) z^{m+1} \partial_{z} a^{*}(z)+\frac{\alpha}{2} z^{m+1}: b(z) \widehat{\Phi}_{x}^{W}(z):+(m+1) \Delta(\mu, \alpha) z^{m} \widehat{\Phi}_{x}^{W}(z) .
$$

Demonstração.

$$
\begin{aligned}
{\left[L_{m}, \widehat{\Phi}_{x}^{W}(z)\right]=} & {\left[\sum_{n \in \mathbb{Z}}(n-m) a_{n} a_{m-n}^{*}+\frac{1}{4}\left(\sum_{n \in \mathbb{Z}}: b_{n} b_{m-n}:\right)-\frac{\mu}{2}(m+1) b_{m}, \widehat{\Phi}_{x}^{W}(z)\right] } \\
= & \sum_{n \in \mathbb{Z}}(n-m)\left(\left[a_{n}, \widehat{\Phi}_{x}^{W}(z)\right] a_{m-n}^{*}+a_{n}\left[a_{m-n}^{*}, \widehat{\Phi}_{x}^{W}(z)\right]\right) \\
& +\frac{1}{4}\left[\sum_{n \in \mathbb{Z}}: b_{n} b_{m-n}:, \widehat{\Phi}_{x}^{W}(z)\right]-\frac{\mu}{2}(m+1)\left[b_{m}, \widehat{\Phi}_{x}^{W}(z)\right] \\
= & \widehat{\Phi}_{f x}^{W}(z) \sum_{n \in \mathbb{Z}}(n-m) a_{m-n}^{*} z^{n}-\frac{\mu}{2}(m+1)\left[b_{m}, \widehat{\Phi}_{x}^{W}(z)\right] \\
& +\frac{1}{4}\left[\sum_{n \in \mathbb{Z}}: b_{n} b_{m-n}:, \widehat{\Phi}_{x}^{W}(z)\right] \\
= & \widehat{\Phi}_{f x}^{W}(z) z^{m+1} \partial_{z} a^{*}(z)-\frac{\mu}{2}(m+1) z^{m} \widehat{\Phi}_{h x}^{W}(z)+\frac{1}{4}\left[\sum_{n \in \mathbb{Z}}: b_{n} b_{m-n}:, \widehat{\Phi}_{x}^{W}(z)\right] \\
= & \widehat{\Phi}_{f x}^{W}(z) z^{m+1} \partial_{z} a^{*}(z)-\frac{\mu \alpha}{2}(m+1) z^{m} \widehat{\Phi}_{x}^{W}(z)+\frac{1}{4}\left[\sum_{n \in \mathbb{Z}}: b_{n} b_{m-n}:, \widehat{\Phi}_{x}^{W}(z)\right]
\end{aligned}
$$

Desta forma, precisamos apenas calcular $\left[\sum_{n \in \mathbb{Z}}: b_{n} b_{m-n}:, \widehat{\Phi}_{x}^{W}(z)\right]$.

$\sum_{n \in \mathbb{Z}}\left[: b_{n} b_{m-n}:, \widehat{\Phi}_{x}^{W}(z)\right]=2 \sum_{n>m}\left[b_{m-n} b_{n}, \widehat{\Phi}_{x}^{W}(z)\right]+\sum_{i=0}^{m}\left[b_{m-i} b_{i}, \widehat{\Phi}_{x}^{W}(z)\right]$

$=2 \sum_{n>m} b_{m-n}\left[b_{n}, \widehat{\Phi}_{x}^{W}(z)\right]+2 \sum_{n>m}\left[b_{m-n}, \widehat{\Phi}_{x}^{W}(z)\right] b_{n}$

$+\sum_{i=0}^{m} b_{m-i}\left[b_{i}, \widehat{\Phi}_{x}^{W}(z)\right]+\sum_{i=0}^{m}\left[b_{m-i}, \widehat{\Phi}_{x}^{W}(z)\right] b_{i}$

$=2 z^{m+1}\left(\sum_{n>m} b_{m-n} z^{n-m-1} \widehat{\Phi}_{h x}^{W}(z)+\widehat{\Phi}_{h x}^{W}(z) \sum_{n>m} b_{n} z^{-n-1}\right)$

$+\left(\sum_{i=0}^{m} b_{m-i} z^{i} \widehat{\Phi}_{h x}^{W}(z)+\widehat{\Phi}_{h x}^{W}(z) \sum_{i=0}^{m} b_{i} z^{m-i}\right)$

$=2 z^{m+1}\left(\sum_{n<0} b_{n} z^{-n-1} \widehat{\Phi}_{h x}^{W}(z)+\widehat{\Phi}_{h x}^{W}(z) \sum_{n \geq 0} b_{n} z^{-n-1}\right)-2 z^{m+1} \widehat{\Phi}_{h x}^{W}(z) \sum_{n=0}^{m} b_{n} z^{-n-1}$

$+z^{m+1} \sum_{i=0}^{m} b_{m-i} z^{-i-1} \widehat{\Phi}_{h x}^{W}(z)+z^{m+1} \widehat{\Phi}_{h x}^{W}(z) \sum_{i=0}^{m} b_{i} z^{-i-1}$ 
Assim, temos:

$$
\begin{aligned}
\sum_{n \in \mathbb{Z}}\left[: b_{n} b_{m-n}:, \widehat{\Phi}_{x}^{W}(z)\right] & =2 z^{m+1}: b(z) \widehat{\Phi}_{h x}^{W}(z):+\sum_{i=0}^{m} b_{m-i} z^{m-i} \widehat{\Phi}_{h x}^{W}(z)-\widehat{\Phi}_{h x}^{W}(z) \sum_{i=0}^{m} b_{i} z^{m-i} \\
& =2 z^{m+1}: b(z) \widehat{\Phi}_{h x}^{W}(z):+\sum_{i=0}^{m} z^{m-i}\left[b_{i}, \widehat{\Phi}_{h x}^{W}(z)\right] \\
& =2 z^{m+1}: b(z) \widehat{\Phi}_{h x}^{W}(z):+\sum_{i=0}^{m} z^{m} \widehat{\Phi}_{h^{2} x}^{W}(z) \\
& =2 z^{m+1}: b(z) \widehat{\Phi}_{h x}^{W}(z):+(m+1) z^{m} \widehat{\Phi}_{h^{2} x}^{W}(z) \\
& =2 \alpha z^{m+1}: b(z) \widehat{\Phi}_{x}^{W}(z):+(m+1) \alpha^{2} z^{m} \widehat{\Phi}_{x}^{W}(z)
\end{aligned}
$$

e, desta forma,

$$
\left[L_{m}, \widehat{\Phi}_{x}^{W}(z)\right]=\widehat{\Phi}_{f x}^{W}(z) z^{m+1} \partial_{z} a^{*}(z)+\frac{\alpha}{2} z^{m+1}: b(z) \widehat{\Phi}_{x}^{W}(z):+(m+1) \Delta(\mu, \alpha) z^{m} \widehat{\Phi}_{x}^{W}(z) .
$$




\section{Capítulo 5}

\section{Considerações finais}

Nesta seção faremos uma breve introdução aos problemas que pretendemos estudar futuramente dando prosseguimento ao que já fizemos no doutorado. Primeiramente tentaremos estender os resultados expostos no capítulo 3 para o caso das álgebras de Lie toroidais no caso dos módulos intermediários de Wakimoto para, em seguida, verificar se faz sentido ou não sua generalização para módulos J-intermediários de Wakimoto. Também tentaremos estender os resultados do capítulo 4 para $\widehat{\mathfrak{s l}}(n, \mathbb{C})$ seguindo [EFK98]. Certamente, tais problemas podem ser abordados para outras álgebras de Lie sendo tal estudo também de nosso interesse.

Para começar, as álgebras de Lie toroidais foram inicialmente introduzidas em [MRY90] como uma generalização natural das álgebras de Lie afins. Álgebras toroidais podem ser vistas como iterações das álgebras de Loop em várias variáveis que comutam. Uma grande motivação para o estudo de álgebras de Lie toroidais é seu potencial de aplicações em Matemática e Física. Por exemplo, um dos cociclos usados na construção de álgebras de Lie afins toroidais estendidas é também usada no estudo de Y. Billig de equação hidrodinâmica magnética com tensor pressão assimétrico (ver [Bi107] e [Bil05]). Além disso, Billig e, independentemente Iohara, Saito, e Wakimoto (ver [Bi199] e [ISW99]) derivou das equações bilineares de Hirota relações tanto principais quanto homogêneas de representações de operadores de Vertex de álgebras de Lie 2-toroidais do tipo $A_{l}, D_{l}, E_{l}$. Por isso, nosso interesse em estudar uma realização de módulos intermediários de Wakimoto para a álgebra de Lie toroidal $\widehat{\mathfrak{s l}}(n, \mathbb{C})$.

Lembremos que representações de álgebras de Lie e suas generalizações são uma das áreas mais importantes e ativas da teoria de representações com uma variedade ampla de resultados e aplicações. A teoria das álgebras de Kac-Moody e suas representações é bastante complexa, com estruturas que relacionam diversas áreas, tais como teoria quântica de campos, sistemas integráveis, álgebras e grupos quânticos, geometria algébrica, teoria dos números, correspondência geométrica de Langlands, etc. Por esta razão, as álgebras de Lie de Kac-Moody, que foram introduzidas em 1967 por V. Kac e R. Moody, se tornaram uma área de estudo importante na Matemática e na Física.

As álgebras de Lie afins são as mais estudadas das álgebras de Lie de Kac-Moody de dimensão infinita, possuindo interessantes aplicações, sendo sua teoria de representações muito mais rica que as das álgebras de Lie de dimensão finita. As álgebras de Lie afins possuem módulos contendo tanto espaços de peso de dimensão finita quanto infinita, algo que não ocorre no caso de dimensão finita. Tais representações surgem partindo-se de 
partições não-standard do sistema de raízes, isto é, partições que não são equivalentes sobre o grupo de Weyl das partições standard em raízes positivas e negativas (ver [DFG09]). Para álgebras de Lie afins, há sempre uma quantidade finita de classes de equivalência de tais partições não-standard (ver [Fut97]). A cada partição corresponde uma subálgebra de Borel, a qual pode-se formar representações obtendo os módulos denominados do tipo Verma, os quais foram inicialmente estudados por Jakobsen e Kac [JK89] e por Futorny [Fut96], [Fut97]. Resultados sobre a estrutura de módulos tipo Verma podem ser encontrados em [Cox94], [Fut91] e [FS93].

Uma das razões da popularidade das álgebras de Kac-Moody afins é a existência de realizações concretas dessas álgebras. Em particular, uma realização muito útil é dada pela extensão central das álgebras de "Loop", i.e., a extensão central do produto tensorial de álgebras simples por polinômios de Laurent. Usando esta realização, pode-se obter informação sobre dimensões de espaços de raízes da álgebra junto com aplicações de álgebras afins à teoria das funções theta, às formas modulares, aos operadores de Vertex, à correspondência Bóson-Férmion e aos sólitons.

As álgebras de Vertex vêm sendo, nas últimas décadas, uma importante ferramenta em diversos assuntos da Física e da Matemática como, por exemplo, na representação de álgebras de dimensão infinita e na teoria de grupos finitos. Uma álgebra de Vertex é basicamente, um espaço vetorial $\mathrm{V}$ onde a cada elemento se corresponde uma distribuição formal com certos axiomas satisfeitos. A primeira definição histórica foi dada em [B86] para superálgebras de Lie conformes. As (super)álgebras conformes possuem, em particular, uma conexão fascinante com os Hamiltonianos na teoria das "equações nãolineares evolucionárias". De fato, a primeira aparição na literatura de estruturas do tipo conforme antecedeu a descoberta das álgebras de Vertex e chegou ao Cálculo de Variações [GD79]. A teoria das álgebras conformes tem sido muito utilizada na tentativa de resolver o problema da classificação de álgebras de Lie de dimensão infinita. Cerca de 40 anos atrás, V. Kac propôs (e parcialmente resolveu) o problema da classificação das álgebras de Lie simples Z-graduadas de Gelfand-Kirillov de dimensão finita ([Kac68]). Este problema foi completamente resolvido por Mathieu em [M86], [M086] e [M92].

Os novos desenvolvimentos na geometria não-comutativa e física-matemática sugeriram a necessidade de estudar módulos de dimensão infinita, tais como módulos de GelfandTsetlin ([Z73]) e módulos de Harish-Chandra ([D74]). A estrutura destes módulos no caso de álgebras de dimensão finita está bem entendida. Certas categorias de tais módulos levaram a teoria de álgebras projetivamente estratificadas à teoria de representações. Por outro lado, a teoria de tais representações está desenvolvida muito pouco no caso de álgebras de Kac-Moody afins. Um dos problemas principais é a construção das realizações destas representações em operadores de Vertex e o desenvolvimento da teoria de álgebras de Vertex generalizadas que correspondem a tais realizações. Já os primeiros exemplos de realizações de álgebras de Lie em operadores diferenciais num espaço de Fock levaram a teoria dos módulos de Wakimoto para álgebras de Kac-Moody afins que foi desenvolvida por H. Jakobsen e V. Kac [JK89], M. Wakimoto [?], B. Feigin e E. Frenkel [?], D. Bernard e G. Felder [BF90].

Algumas construções particulares foram recentemente obtidas por Y. Billig, A. Molev, R. Zhang [BMZ08], B. Cox, V. Futorny [CF04], [CF05], e R.A. Martins [Mar12], generalizando as construções clássicas de módulos de Wakimoto. Em particular, os resultados do trabalho [BMZ08] tem aplicações importantes na teoria de equações diferenciais. No trabalho [CF04], foi definida a classe de módulos do tipo Wakimoto 
torcidos para álgebras de Kac-Moody afins $A_{n}^{(1)}$. Estes módulos fornecem uma série de realizações novas do tipo bóson para álgebras afins do tipo A.

Todos estes resultados sugerem a necessidade de se estudar a teoria de estruturas de Vertex relacionadas as realizações de módulos de dimensão infinita de álgebras de KacMoody afins.

Desta forma, entre os nossos maiores objetivos estão a construção e o estudo de realizações do tipo Vertex para módulos de Verma generalizados sem peso máximo sobre as álgebras de Lie simples de dimensão finita e sobre as álgebras afins e o desenvolvimento da teoria de álgebras de Vertex generalizadas associadas com as realizações de módulos de Verma generalizados sem peso máximo. A realização de módulos de Verma generalizados sem peso máximo em operadores do tipo Vertex poderá trazer novas informações sobre a estrutura destes módulos e, portanto, seria uma contribuição importante à teoria de representações. Além disso, espera-se que o estudo de estruturas de Vertex relacionadas aos tais módulos leve a uma teoria nova de álgebras de Vertex generalizadas. 


\section{Referências Bibliográficas}

[B86] Borcherds R., Vertex algebras, Kac-Moody algebras, and the Monster, Proc. Natl. Acad. Sci. USA, 83: 3068-3071, 1986.

[BBFK11] Bekkert, V., Benkart, G., Futorny, V., Kashuba, I., New Irreducible modules for Heisenberg and Affine Lie Algebras, preprint, 2011.

[BF90] Bernard D., Felder G., Fock representations and BRST cohomology in sl(2) current algebra, Comm. Math. Phys., 127(1):145-168, 1990.

[Bil05] Billig Y., Magnetic hydrodynamics with asymmetric stress tensor, J. Math. Phys., 46(4):043101, 13, 2005.

[Bil07] Billig Y., Representations of toroidal extended affine Lie algebras, J. Algebra, 308(1):252-269, 2007.

[Bi199] Billig Y., An extension of the Korteweg-de Vries hierarchy arising from a representation of a toroidal Lie algebra, J. Algebra, 217(1):40-64, 1999.

[BMZ08] Billig Y., Molev A., Zhang R., Differential equations in vertex algebras and simple modules for the Lie algebra of vector fields on a torus, Advances in Math., 218:1972-2004, 2008.

[C86] Chari V., Integrable representations of affine Lie Algebras, Invent. Math., 85:317-335, 1986.

[CF04] Cox B., Futorny V., Intermediate Wakimoto modules for affine sl( $n+1)$, J. Phys. A, 37(21): 5589-5603, 2004.

[CF05] Cox B., Futorny V., Structure of Intermediate Wakimoto modules, J. Algebra, 306: $682-702,2006$

[CFMar12] Cox, B., Futorny, V., Martins, R.A., Virasoro action on imaginary Verma modules and the operator form of the KZ-equation, preprint.

[Cox05] Cox, B., Fock Space Realizations of Imaginary Verma Modules, Algebr. Represent. Theory, 8:173-206, 2005.

[Cox94] Cox B., Verma modules induced from nonstandard Borel subalgebras, Pacific J. Math., 165: 269-294, 1994. 
[CP088] Chari V., Pressley A., Integrable representations of Kac-Moody algebras: Results and open problems, Infinite dimensional Lie Algebras and Groups, Adv. series in Math. Physics (ed. V. Kac), World Scientific, 1988.

[CP88] V. Chari, A. Pressley, Integrable representations of torcido affine Lie algebras, J. Algebra, 113:438-464, 1988.

[D74] Dixmier J., Algebras enveloppantes, Gauthier-Villarrd, Paris, 1974.

[DFG09] Dimitrov I., Futorny V., Grantcharov D., Parabolic sets of roots, Groups, Rings and Group Rings, Contemp. Math., Amer. Math. Soc., Providence, RI, 499:61-73, 2009.

[DGK82] Deodhar V., Gabber O., Kac V., Structure of some categories of representations of infinite-dimensional Lie algebras, Adv. in Math., 45:92-116, 1982.

[DJKM82] Date E.,Jimbo M., Kashiwara M., Miwa T., Transformation groups for soliton equations. Euclidean Lie algebras and reduction of the KP hierarchy, Publ. Res. Inst. Math. Sci. 18:1077-1110, 1982.

[EFK98] Etingof P.I., Frenkel I.B., Kirillov Jr. A.A., Lectures on representation theory and Knizhnik-Zamolodchikov equations, 58, Mathematical Surveys and Monographs. AMS, 1998.

[FF90] Feigin B., Frenkel E., Affine Kac-Moody algebras and semi-infinite flag manifolds, Com. Math. Phys., (1):161-189, 1990.

[FF090] Feigin B., Frenkel E., Representations of affine Kac-Moody algebras and bosonization, In Physics and Mathematics of Strings, World Scientific, Teaneck, NJ, 271-316, 1990.

[FF88] Feigin B., Frenkel E., A family of representations of affine Lie algebras, Uspekhi Mat. Nauk, 43(5(263)):227-228, 1988.

[FK09] Futorny V., Kashuba I., Induced modules for Kac-Moody Lie algebras, SIGMA - Symmetry, Integrability and Geometry: Methods and Applications 5:026, 2009 .

[FKM01] Futorny V., Konig S., Mazorchuk V., Categories of induced modules for Lie algebras with triangular decomposition, Forum Math., 13(5):641-661, 2001.

[FS93] Futorny V., Saifi H., Modules of Verma type and new irreducible representations for affine Lie algebras, Representations of algebras (Ottawa, ON, 1992), CMS Conf. Proc., Amer. Math. Soc., Providence, RI, 14:185-191, 1993.

[Fut90] Futorny V., Parabolic partitions of root systems and corresponding representations of affine Lie algebras, Root systems, Representations and Geometries, Inst. math. Acad. Sci. Ukraine. Preprint 90.8, Kiev, 30-39, 1990. 
[Fut91] Futorny V., The graded representations of affine Lie algebras, Suppl. Rend. Circolo Matem. di Palermo, serie II, 26:155-161, 1991.

[Fut92] Futorny V., The parabolic subsets of root systems and corresponding representations of affine Lie algebras, Proceedings of the International Conference on Algebra, Part 2 (Novosibirsk, 1989), Contemp. Math., Part 2, Amer. Math. Soc., Providence, RI, 131:45-52, 1992.

[Fut94] Futorny V., Imaginary Verma modules for affine Lie algebras, Canad. Math. Bull., 37(2):213-218, 1994.

[Fut96] Futorny V., Irreducible non-dense $A_{1}^{(1)}$-módulos, Pacific J. Math., 172:83-99, 1996.

[Fut97] Futorny V., Representations of Affine Lie Algebras, Queen's Papers in Pure and Applied Math., Queen's University, Kingston, ON, 106, 1997.

[FS93] Futorny V., Saifi H., Modules of Verma type and new irreducible representations for affine Lie algebras, Representations of algebras (Ottawa, ON, 1992), Amer. Math. Soc., Providence, RI, 185-191, 1993.

[FZ01] Frenkel E.,Ben-Zvi D., Vertex algebras and algebraic curves, Math. Surveys and Monographs, AMS 83, 2001.

[GD79] Gelfand I., Dorfman I., Hamiltonian operators and algebraic structures related to them, Funct. Anal. and Appl. 13:248-262, 1979.

[ISW99] Iohara K., Saito Y., Wakimoto M., Hirota bilinear forms with 2-toroidal symmetry, Phys. Lett. A, 254(1-2):37-46, 1999.

[JK85] Jakobsen H.P., Kac V.G., A new class of unitarizable highest weight representations of infinite-dimensional Lie algebras, Nonlinear equations in classical and quantum field theory (Meudon/Paris, 1983/1984), Springer, Berlin, 1-20, 1985.

[JK89] Jakobsen H.P., Kac V.G., A new class of unitarizable highest weight representations of infinite dimensional Lie algebras, J. Funct. Anal., 82:6990, 1989.

[Kac68] Kac V., Simple irreducible graded Lie algebras of finite growth., Math. USSRIzvestija 2, 1271-1311, 1968.

[Kac90] Kac V., Infinite dimensional Lie algebras, Cambridge University Press, third edition, 1990.

[Kac98] Kac V., Vertex algebra for beginners, Amer. Math. Soc., Providence, RI, second edition, 1998.

[KKLW81] Kac V., Kazhdan D., Lepowsky J., Wilson R., Realization of the basic representations of the Euclidean Lie algebra, Adv. in Math., 42:83-112, 1981. 
[KR87] Kac V.G., Raina A.K., Bombay lectures on highest weight representations of infinite dimensional Lie algebras, World Scientific Publishing Co., Inc., Teaneck, NJ, 1987.

[LW78] Lepowski J., Wilson R.L., Constructions of the affine Lie algebra $A_{1}^{(1)}$, Comm. Math. Phys. 62:179-194, 1978.

[M86] Mathieu O., Sur un problème de V.G.Kac: la classificacion de certaines algèbres de Lie graduèes simples, J. Algebra 102:505-536, 1986.

[M086] Mathieu O., Classification des algèbres de Lie graduèes simples de croissance $\leqslant 1$, Invent. Math., 86:371-426, 1986.

[M92] Mathieu O., Classification of simple graded Lie algebras of finite growth, Invent. Math. 108:455-519, 1992.

[Mar11] Martins R.A., Free field realizations of certain modules for affine Lie algebra $\widehat{\mathfrak{s l}}(n, \mathbb{C})$, Algebra Discrete Math., Volume 12 , Number 1., 28-52, 2011.

[Mar12] Martins R.A., I-Intermediate Wakimoto Modules, Comm. Algebra, to appear.

[MRY90] Moody R.V., Rao S.E, Yokonuma T., Toroidal Lie algebras and Vertex representations, Geom. Dedicata, 35(1-3):283-307, 1990.

[R89] Rao S.E, A new class of unitary representations for Affine Lie Algebras, J. Algebra, 120(1):54-73, 1989.

[R93] Rao S.E., On representations of Loop Algebras, Comm. Algebra, 21(6):21312153, 1993.

[RW82] Rocha A., Wallach N., Projective modules over graded Lie Algebras I, Math. Z., 180:151-177, 1982.

[SM99] San Martin, L. A. B., Álgebras de Lie., 1. ed. Campinas: Unicamp, 1999.

[Wak86] Wakimoto M., Fock representations of the affine Lie algebra $A_{1}^{(1)}$, Comm. Math. Phys., 104(4):605-609, 1986.

[War93] Warner S., Topological Rings, Mathematics Studies , North-Holland, 178, 1993.

[Z73] Zelobenko D., Compact Lie groups and their representation, Translated from the Russian by Israel Program for Scientific Translations. Translations of Mathematical Monographs, Vol. 40. American Mathematical Society, Providence, R.I., 1973. 